\title{
Channelisation of Noise through a Rhythmic Grid: Brutalist Mechatronic Sound-sculpture
}

\author{
by \\ Mo H. Zareei
}

A thesis

submitted to Victoria University of Wellington

in fulfilment of the requirements for the degree of

Doctor of Philosophy

Victoria University of Wellington

2016 


\section{Dr. Ajay Kapur \\ Prof. Dale A. Carnegie \\ Dr. Dugal McKinnon}


"There is no order that does not contain disorder within itself, and undoubtedly there is no disorder incapable of creating order."

Jacques Attali 


\begin{abstract}
The aim of this thesis is to provide accessibility and appreciation for sounds that are conventionally perceived as non-musical or "noise". Ordering the noise on a grid of metric rhythms, and underlining its materiality through an audiovisual mode of expression are the two main strategies employed. Using the medium of mechatronics, mechanically generated sonic by-products of technological developments are chosen as the focus sonic material. As a result, the output of this research extends what is known as glitch music outside the territory of amplified sound, to a realm where noise is created physically and acoustically.

Based on these objectives, and following an investigation on the use of mechatronics in contemporary sound-based art, an ensemble of mechatronic sound-sculptures is designed and developed. Varying in terms of material, sound-generating mechanism, and sonic quality, the ensemble is divided into three different instrument-types, each of which is introduced, thoroughly described, and sonically evaluated. Next, three new audiovisual works are developed and realised utilising the mechatronic sound-sculptures, in order to turn into practice the ideas explored in this research. These compositions - which are all exhibited in competitive international symposiums - undertake the integration of mechatronics in three areas of sonic arts that are interconnected with the sound-sculptures.

Furthermore, this thesis also establishes an aesthetic framework that formalises a significant body of contemporary sound art and music that, prior to this work, had suffered academic inattention. Probing the various parallels between the ideas developed in this thesis and Brutalist architecture, 'sound-based brutalism' is coined and formulated as an aesthetic underpinning for not only the academically marginalised works discussed, but also the work of the author. Lastly, two audiovisual projects (a performance and a series of ten installation pieces) are developed using the entire mechatronic sound-sculpture series in an effort to realise 'sound-based brutalism'.
\end{abstract}




\section{Acknowledgments}

It is impossible to express in one or two pages, my deep gratitude to the very many who helped, supported, and inspired me in the path that has led me here. Therefore, the following is only a non-exhaustive list of the people without whom this document would not exist.

First and foremost, thanks to my incredible PhD advisors Dr. Ajay Kapur, Prof. Dale Carnegie, and Dr. Dugal McKinnon. Thanks to Ajay, whose role in my life over the past six years has gone above and beyond being my university teacher. From his very first extensive and enthusiastic response to my email enquiry about the Music Technology programme at CalArts, all the way to this very moment, Ajay has continuously helped, motivated, and encouraged me in achieving the unthinkable. Thank you, Ajay, for believing in me, for being a great mentor, and even a greater friend.

I cannot be thankful enough to Dale - possibly one of the busiest professionals I have ever met - for the hours and hours of meetings, planning, and discussions, and for going over every single paragraph I have produced as a $\mathrm{PhD}$ student. Thank you, Dale, for all your guidance, discipline, diligence, and openness.

Countless thanks to Dugal, whose vast knowledge and insightful feedback played a crucial role in supporting my academically dangerous ideas! Thank you Dugal, for all the fulfilling discussions, critical debates, and for all your precious input.

Without the assistance of the faculty and staff at Victoria University's School of Engineering and Computer Science and the New Zealand School of Music, completion of this $\mathrm{PhD}$ would not have been possible. In particular, many thanks to Tim Exley, for his allencompassing technical expertise and his endless and selfless help and advising. Same goes for Sean Anderson, Jason Edwards, and James McVay: thank you all for the nonstop help. Thanks to Michael Norris and Ted Apel for their valuable feedback and critical thoughts. Thanks also to Victoria University of Wellington Image Services and Warren Butcher. 
I would like to extend my thanks to my fellow PhD colleagues at the Sonic Engineering Lab for Creative Technologies, not only for their constant support, but also for putting up with all the noise! Special thanks goes to my dear friend, tutor, critic, reviewer, validator, supporter, helper, and collaborator, Jim Murphy. Thank you, Jim, for every single bit (and byte!) of your help, knowledge, and support.

I am extremely appreciative of my outstanding teachers at CalArts and their significant impact on my artistic and academic career. Above all, I would like to mention Martijn Zwartjes, Rob Wannamaker, Ulrich Krieger, and Barry Schrader, who shaped my appreciation of sound, noise, silence, and music, and taught me in the realest form, how to think critically and outside the box. I am also grateful to my Physics professor at Shahid Beheshti University of Tehran, Dr. Sepangi, and my high school math teacher, Mr. Molazadeh, for bringing into life, the very textbook definition of a selfless, devoted, and loving teacher.

I owe this thesis also to the remarkable artists who have continuously inspired and influenced me. To name a few: Carsten Nicolai, Ryoji Ikeda, Olaf Bender, Nicolas Bernier, Martin Messier, Zimoun, Pe Lang, Mika Vainio, Robert Henke.

To my many friends who are scattered all around world, from my beloved Tehran, to the not-so-great Valencia CA, to beautiful Wellington and beyond: I cannot possibly select only a few to name here. Your support and love has been key in making me who I am. Thank you, thank you, thank you!

To Rosie, for all the kindness and encouragement, for celebrating every tiny little academic achievement of mine, and for all the goodness that you poured into my otherwisewould-have-been-very-hard-and-stressful final year of PhD! Thank you, azizam!

And finally, endless thanks to my beloved family. Thank you maman jan, and thank you baba jan. Thank you for your openness, for supporting me in my crazy decision to move to America to start an undergrad degree in music after finishing my Physics degree, and remaining hopeful and optimistic that it would get somewhere. And thank you to my absolute dearest Hoda, who turned from a lovely little teenager into a beautiful smart young woman, while her only sibling was away on his journey. Thank you all for your patience, and for all the sacrifices, financial and emotional, that you made for me. I cannot even begin to explain how much it all means. 


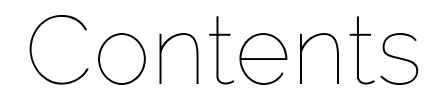

Acknowledgments

Chapter 1 Introduction

1.1 Overview 1

1.2 Noise on the Grid: Motivation 2

1.2.1 Noise 2

1.2.2 Pulse 5

1.2.3 Pulse-based Noise 6

$\begin{array}{lll}1.3 & \text { Objectives } & 7\end{array}$

$\begin{array}{lll}1.4 & \text { Thesis Outline } & 10\end{array}$

1.5 Summary of Contributions 11

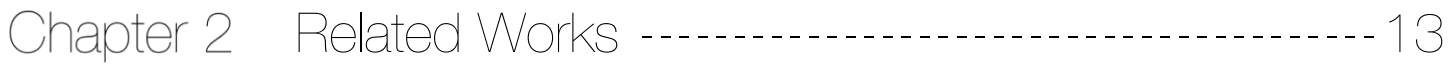

2.1 Overview 13

2.2 Noise on the Grid 14

2.2.1 Background 14

2.2.2 Contemporary Pulse-based Glitch $\quad 15$

2.2.3 Discussion 18

2.3 Mechatronic Sound Art $\quad 20$

2.3.1 Overview 20

2.3.2 Musical Robotics 20

2.3.3 A New Classification 23

2.3.4 Mechatronic Sound-objects 24

2.3.5 The New Generation 26

$\begin{array}{ll}2.3 .6 & \text { Discussion } \\ & 29\end{array}$ 
Chapter 3 Mechatronic Sound-sculptures ..................... 33

\subsection{Overview 33}

3.2 Rasper 35

3.2.1 Design 36

3.2.2 System Overview

3.2.3 Evaluation $\quad 42$

3.2.4 Summary 49

$\begin{array}{lll}3.3 & \text { Rippler } & 49\end{array}$

3.3.1 Design $\quad 50$

3.3.2 System Overview

3.3.3 Evaluation 56

3.3.4 Summary 62

$\begin{array}{lll}3.4 & \text { Mutor } & 63\end{array}$

3.4.1 Design 63

3.4.2 System Overview 65

$\begin{array}{ll}\text { 3.4.3 Evaluation } & 67\end{array}$

3.4.4 Summary 72

3.5 Collective Evaluation $\quad 72$

3.5.1 Relative Frequency Range 72

3.5.2 Relative Loudness 73

$\begin{array}{lll}3.6 & \text { Summary } & 75\end{array}$

Chapter 4 Compositional Approaches.....................79

\section{$\begin{array}{lll}4.1 & \text { Overview } & 79\end{array}$}

4.2 Remodelling Early Minimalist Composition 80

4.2.1 Introduction: Minimalist Music 80

4.2.2 Pulse Music of Steve Reich 81

4.2.3 Rasping Music: an Audiovisual Composition 82 
4.2.4 Conclusion 86

4.3 Physical Sonification of Cellular Automata 87

4.3.1 Introduction: Algorithmic Composition 87

4.3.2 Cellular Automata Music 89

4.3.3 NOISE SQUARE: an Audiovisual Composition 92

4.3.4 Conclusion 95

4.4 Physical Re-sonification of Urban Noise 96

4.4.1 Introduction: Aesthetics of Urban Soundscape 96

4.4.2 Urban Soundscape: From Attention to Appreciation 97

4.4.3 complex: an Audiovisual Composition 100

4.4.4 Conclusion 103

$\begin{array}{lll}4.5 & \text { Summary } & 104\end{array}$

Chapter 5 Sound-based Brutalism -......................... 107

5.1 Introduction: an Emergent Aesthetic 107

$\begin{array}{ll}5.1 .1 \text { Overview } & 107\end{array}$

5.1.2 A Need for Further Discussion 108

5.2 Defining the New Aesthetic 110

5.2.1 Inaptitude of "Minimalist" 111

5.2.2 Towards a More Coherent Definition 113

5.3 Brutalism: from Architecture to Sound Art 117

5.3.1 Brutalist Architecture: an Overview 117

5.3.2 Brutalist Sound Art: Realisation 121

5.4 Discussion: Sound-based Brutalism 128

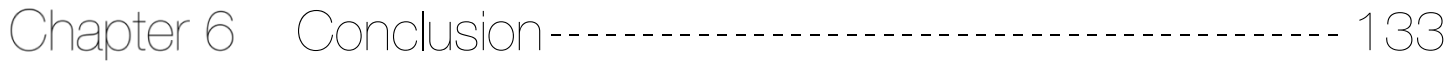

6.1 Summary 133

6.1.1 Motivation / Objectives 133

6.1.2 Background / Related Works 134

6.1.3 Design / Development 134 
6.1.4 Application / Realisation 136

6.1.5 Observation / Reflection 137

6.2 Future Work 138

6.3 Novel Contributions 140

6.3.1 Primary Contributions 140

6.3.2 Research Outputs 140

6.4 Denouement 144

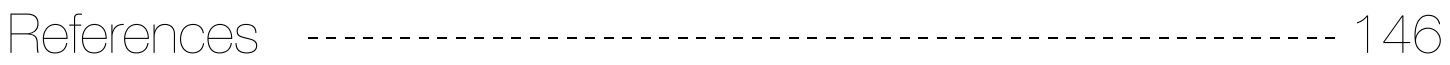

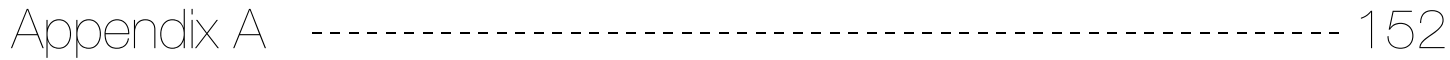

The Driver Board (code) 152

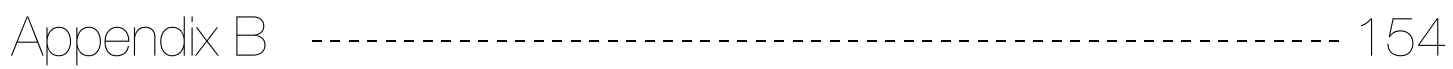

Rasping Music (score) 154

Noise Square (code) 163

$\begin{array}{ll}\text { Complex (code) } & 168\end{array}$

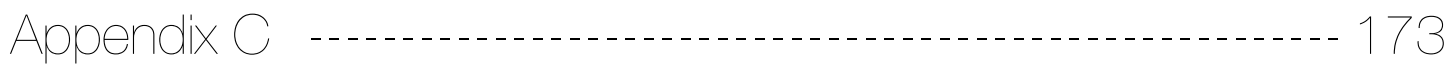

Supplemental Material 173 


\section{List of Figures}

Figure 1-1. Different categories of noise

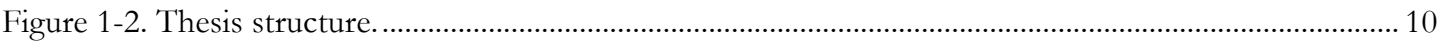

Figure 2-1. Luigi Russolo (left) and his Intonarumori. By Luigi Russolo, via Wikimedia Commons........... 14

Figure 2-2. Nicolas Collins' Sled Dog: hand-scratchable hacked CD player. Photo by Simon Lonergan, courtesy of Nicolas Collins.

Figure 2-3. The development of a two-bar rhythm in Impulse by Carsten Nicolai, transcribed by Adam Collis [20]. Here, the sound material (noise, clicks, and sine tones) are articulated using metric rhythmic patterns.

Figure 2-4. Kapur's MahaDeviBot. Photo by Jim Murphy, courtesy of Ajay Kapur....................................... 21

Figure 2-5. Godfried-Willem Raes and his mechatronic musical instruments. Photo from www.logosfoundation.org, courtesy of Godfried-Willem Raes.

Figure 2-6. Klompen by Trimpin (1990): electronically driven mallets placed in wooden clogs. Photo courtesy of Frye Art Museum and the artist.

Figure 2-8. La chambre des machine by Nicolas Bernier and Martin Messier. Photo courtesy of the artists. ... 26

Figure 2-9. Left: Bernier's Frequencies (a) (2012), photo courtesy of the artist. Right: Messier's Sewing Machine Orchestra (2010), photo courtesy of the artist. 27

Figure 2-10. 216 prepared DC motors, filler wire $1.0 \mathrm{~mm}$ (2009/2010), by Zimoun. Photo courtesy of the artist.

Figure 2-11. Motor Music Test by Daito Manabe. Photo courtesy of the artist. 29

Figure 3-1. Hybridisation of contemporary digital glitch music and mechatronic sound-objects by combining some of their key elements.

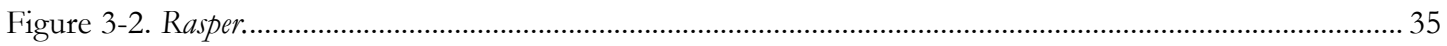

Figure 3-3. Rasper: design sketch, parts, and 3D model. …...................................................................... 36

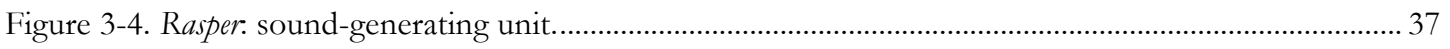

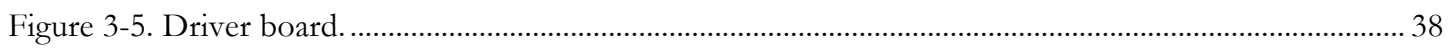

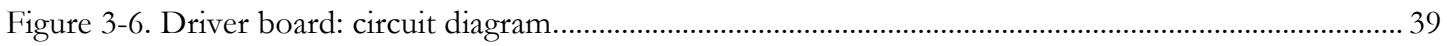

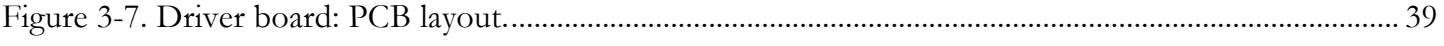

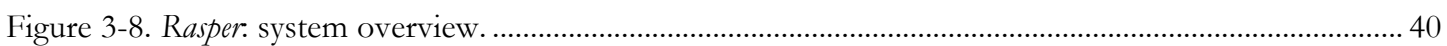

Figure 3-9. Sound-generating mechanism of Rasper (left) contrasted to the one used in a model of

Russolo’s Crackler, developed by New Music Co-op [52] ......................................................... 41

Figure 3-10. Average FFT results of recordings of Rasper at different speeds............................................. 43

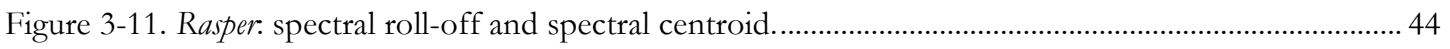

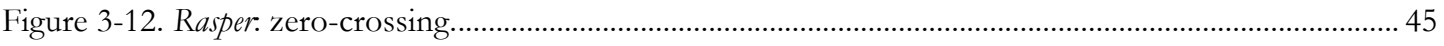

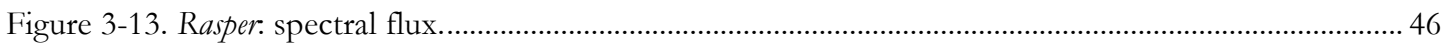


Figure 3-14. Rasper's output generated from a 2-second long input with maximum MIDI velocity values for the motor and the solenoid: waveform and spectrum representations.

Figure 3-15. The full latency $\left(\mathrm{L}_{1}\right)$ is comprised of the software latency $\left(l_{1}\right)$ and the mechanical latency $\left(l_{2}\right) .48$

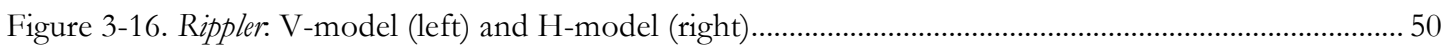

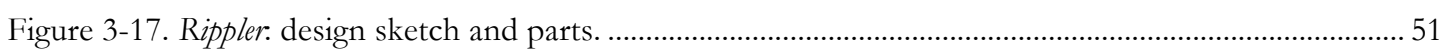

Figure 3-18. Rippler V-model: 3D model (left), design sketch: side and back views (right). .......................... 51

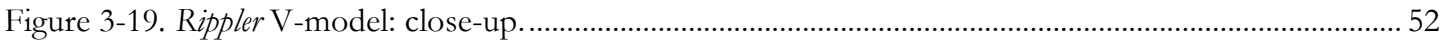

Figure 3-20. Rippler H-model: 3D model (left), design sketch back and top views (right)............................ 52

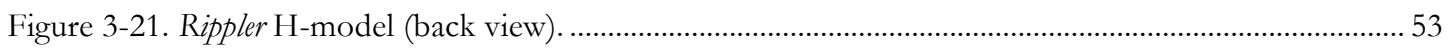

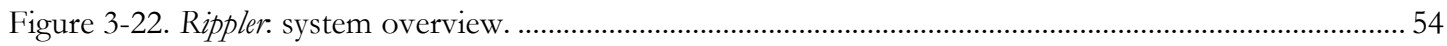

Figure 3-23. Rippler sound-generating mechanism: V-model (left), H-model (right).................................... 55

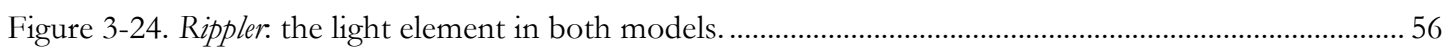

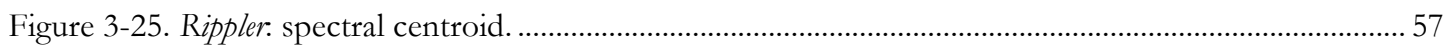

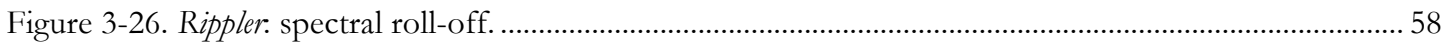

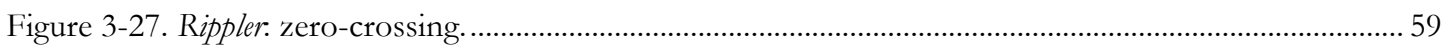

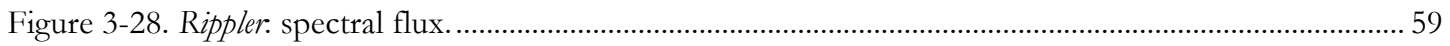

Figure 3-29. Rippler: waveform representation of recordings for a single strike: (a) H-model, single

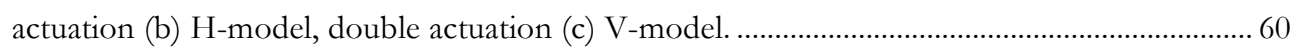

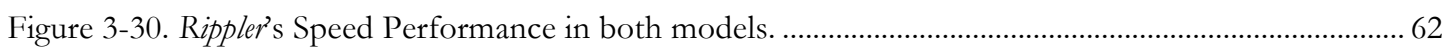

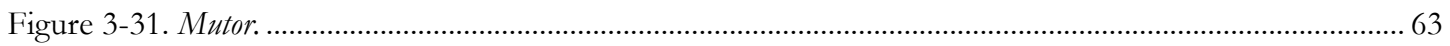

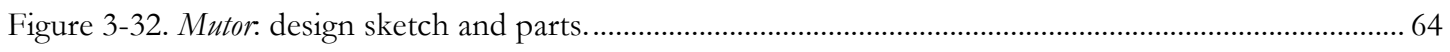

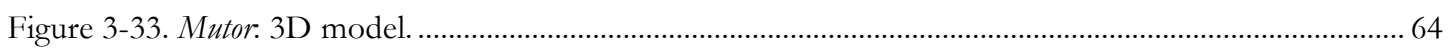

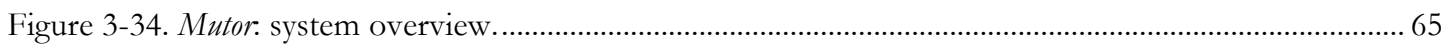

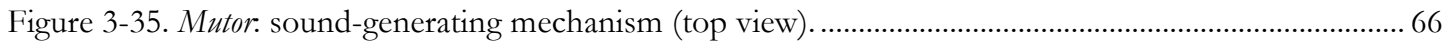

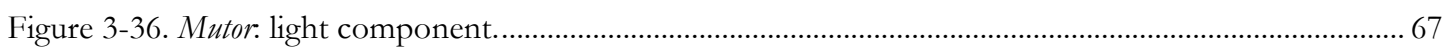

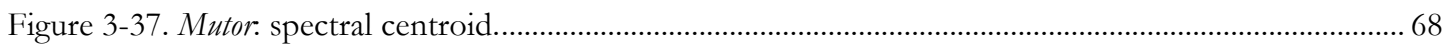

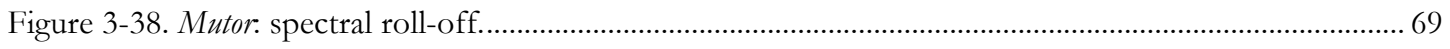

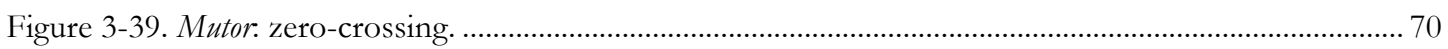

Figure 3-40. Visualisation of a 1-bar long pattern played by Mutor: (a) MIDI, (b) waveform, (c) spectrum.71

Figure 3-41. Spectral centroid of Rasper, Rippler, and Mutor compared...................................................... 73

Figure 3-42. Relative loudness of the sound-sculptures. ........................................................................... 74

Figure 4-1. Rasping Music: installation realisation at Sonic Arts and Engineering Expo 2013, Victoria

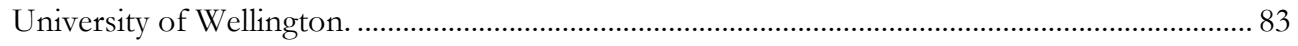

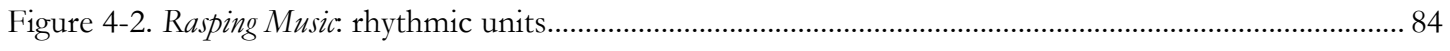

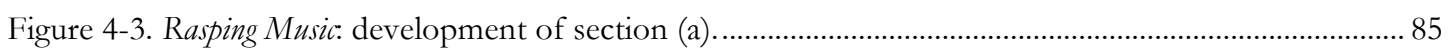

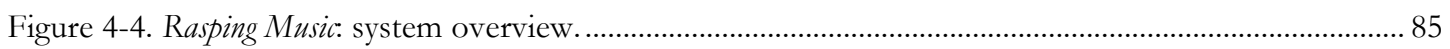


Figure 4-5. Rasping Music: audiovisual installation at Lux 2014, Wellington, New Zealand. 86

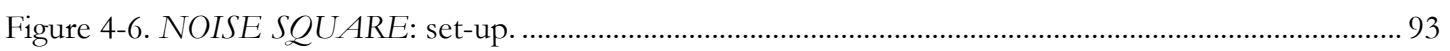

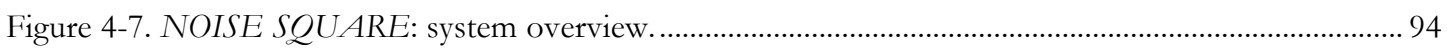

Figure 4-8. NOISE SQUARE: audiovisual installation at Vancouver Art Gallery, ISEA 2015.................. 95

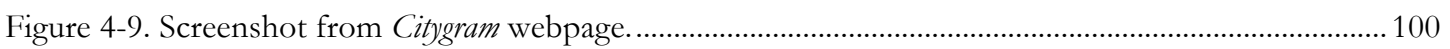

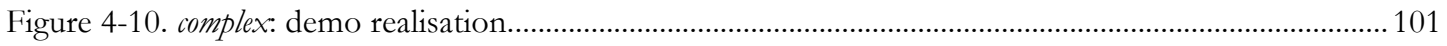

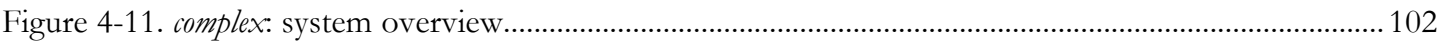

Figure 4-12. complex: exhibition at NYU magnet, ICAD 2014, Brooklyn, NY............................................ 103

Figure 5-1. Top-left: 80 prepared dc motors, cotton balls, cardboard boxes $71 \times 71 \times 71 \mathrm{~cm}$ (2011) by Zimoun. Photo courtesy of the artist. Top-right: Olaf Bender aka Byetone performing live (2008). Photo by Alvaro Fárfan, via www.flickr.com (CC BY-NC 2.0). Bottom-left: Motor Music Test 55 (2013) by Daito Manabe. Photo courtesy of the artist. Bottom-right: test pattern [ $\left.{ }^{\circ} 3\right]$ (2010), photo by Marc Domage, courtesy of Ryoji Ikeda. 116

Figure 5-2. Top-left: Unité d'habitation by Le Corbusier. Photo by Sébastian Pérez-Duarte, via www.flickr.com (CC BY-SA 2.0). Top-right: Salk Institute by Louis Kahn. Photo by Jim Harper, via Wikimedia Commons (CC SA 1.0). Bottom-left: Boston City Hall by Gerhard Kallmann and Michael McKinnell. Photo by Daniel Schwen, via Wikimedia Commons (CC BY-SA 4.0). Bottom-right: Habitat 67 by Moshe Safdie. Photo by Wladyslaw Sojka, via Wikimedia Commons (CC-BY SA 3.0) .................................................................................... 119

Figure 5-3. Ekbatan residential building complex, Tehran. Photo courtesy of Hoda Zareei....................... 120

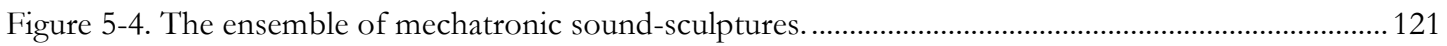

Figure 5-5. The Brutalist Noise Ensemble (2015), audiovisual performance.................................................... 123

Figure 5-6. The Brutalist Noise Ensemble: MIDI notation of a 4-bar long rhythmic pattern composed for Rasper (top), Rippler $\mathrm{H}$ and $\mathrm{V}$ models (middle), and Mutor (bottom)...... 124

Figure 5-7. The Brutalist Noise Ensemble curated by Streaming Museum (a screen shot of Streaming Museum

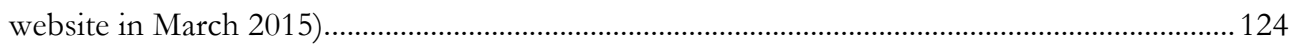

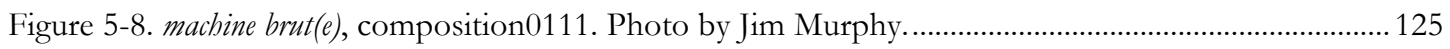

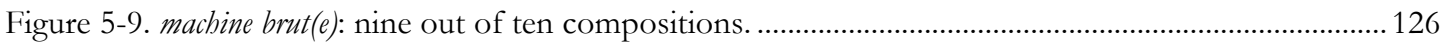

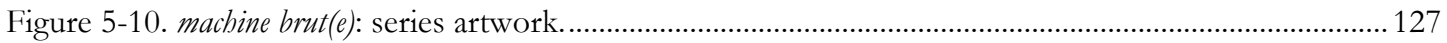




To Hadi, Hamed, and Farhang,

and our wanders in Ekbatan... 




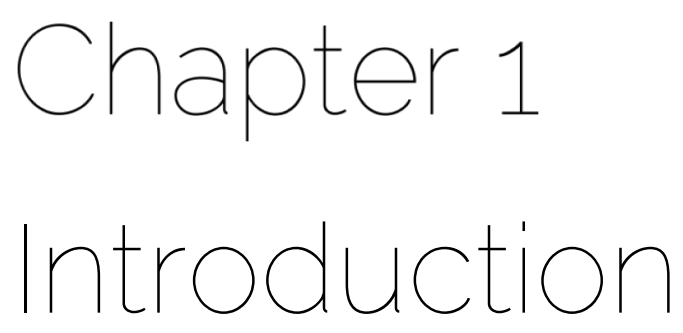

\subsection{Overview}

Referring to sound as 'all that can be heard' and rbythm as 'the formulating impulse behind sound', Henry Cowell understands these as the primary musical elements [1]. The aim of this research is to use rhythms that are simple and metric, in order to give accessibility to sounds that are conventionally interpreted as extra-musical and inaccessible. Therefore, this thesis investigates a state in which Cowell's primary musical elements meet at opposed points: rhythm is regular, simple, and accessible (pulse), whereas sound is irregular, complex, and inaccessible (noise). Accordingly, this research is dedicated to the design and construction of apparatuses that are capable of producing "noise", while temporally regulating this through a grid of pulse-based and metric rhythms. In order to accomplish this, an ensemble of sound-sculptures will be developed that, utilising mechatronics and microcontroller programming, can function both automatically and interactively. With this ensemble, the goal is to reveal the potential aesthetics of the mechanically generated noise using a rhythmic grid, and to bring to the foreground the background noise of contemporary urban technological life. Building upon this concept of "noise on the grid", this thesis also endeavours to establish a clear framework for situating and understanding certain contemporary practices in electronic music and sound art seems largely absent from scholarly literature. This chapter provides the 
background to and motivation for this research, describes its main objectives, and presents an overview of the thesis structure.

\subsection{Noise on the Grid: Motivation}

This section introduces the core concepts behind this research: pulse (that is rhythmic grid), noise, and their fusion.

\subsubsection{Noise}

If the idea of using "noise" in music was gradually introduced via the entrance of percussion instruments into the orchestra during the $19^{\text {th }}$ century, the idea of noise as music was explicitly asserted by Luigi Russolo in his Futurist Manifesto in 1913. Calling for integration of more dissonant, stranger, and harsher sounds into music, Russolo's aim was to "conquer the infinite variety of noise-sounds" [2].

Following Russolo and his Intonarumori (noise-makers), there have been numerous instances of putting sounds conventionally interpreted as non- or extramusical into a musical frame using different methods and techniques. Varying according to the technology and philosophy of the day, this ranges from George Antheil's Ballet Mécanique, to Pierre Schaeffer's musique concrète, John Cage's call for incorporation of "all-sound" [3], Edgar Varèse's and Stockhausen's groundbreaking electronic works, the Industrial music of 1970's and 1980's, Christian Marclay's turntablism, and on to the contemporary pulsebased laptop-produced glitch music.

First, in order to address the questions "why use noise?" and "why work in a rhythmic format?" a working definition of the term "noise" needs to be made. There are different approaches to defining noise and its territory and there are a number of texts that are devoted, fully or partially, to an exploration of these various approaches. ${ }^{1}$ To list

${ }^{1}$ such as Noise/Music: a History by Paul Hegarty, or Audio Culture: Readings on Modern Music edited by Christoph Cox and Daniel Warner. 
those that are most pertinent to the context of this research, noise can be summarised as: irregular vibrations, undesirable sound, or redundant information.

\subsubsection{Irregular Vibrations: Acoustic Noise}

In the first chapter of his classic text on the perceptual basis of music theory, The Sensations of Tone, Hermann Helmholtz argues that the differences between noises and musical tones are rooted in our aural perception, stating that musical tones are perceived as periodic, and noises are perceived as non-periodic motions [4]. This view presents the acoustic definition of noise in which pure noise (white noise) is diametrically contrasted with pure pitch (sine tone). However, in Russolo's view, although irregularity of motions in terms of time and intensity still establishes the difference between "sound" and "noise", it is not "sufficient enough to make a sharp distinction" [2]. As Trevor Wishart suggests, this distinction "is a property of the way we hear rather than of the object itself", and is somehow "arbitrary" [5]. Russolo's and Wishart's views, in fact, reflect the sociocultural definition of noise which, in many cases, is not aligned with the acoustic definition.

\subsubsection{Undesirable and Unpleasant: Sociocultural Noise}

In addition to the acoustic definition, there is a more subjective way of looking at noise: one that varies depending on different sociocultural elements. In this view, noise can be perceived as any aural experience that is annoying or undesirable, depending on the perceiver, time, and the location of the event. This could be the sound of scratching a metal surface with a sharp object, the loud din of a construction operation, distortion effects in grunge music, a piece of atonal music from the early $20^{\text {th }}$ Century, or the quarter-tone intervals of traditional Persian music. Salomé Voegelin refers to the relationship of "noise" and "its hearer" as reciprocal, and - taking a Sartrean direction ${ }^{2}-$ describes noise as "other people's music" [6]. The pioneer Japanese noise musician, Masami Akita (aka Merzbow) declares that if noise means uncomfortable sound, pop music is noise to him [7]. According to Edgar Varèse, "to stubbornly conditioned ears,

\footnotetext{
2 “Hell is other people." - No Exit (1944), Jean-Paul Sartre.
} 
anything new in music has always been called noise". Varèse defines noise as "any sound one doesn't like" [8].

As the figure below suggests, acoustic noise and sociocultural noise can overlap, or exist as completely independent phenomena. Some examples for this could be:

A: The sound of the snare drum in a music concert.

B: Phone ringtone made of a piano recording heard in a library.

C: Static noise while tuning the radio.

Depending on the context in which noise is being defined as such, the same sound can fit into two or all of these categories.

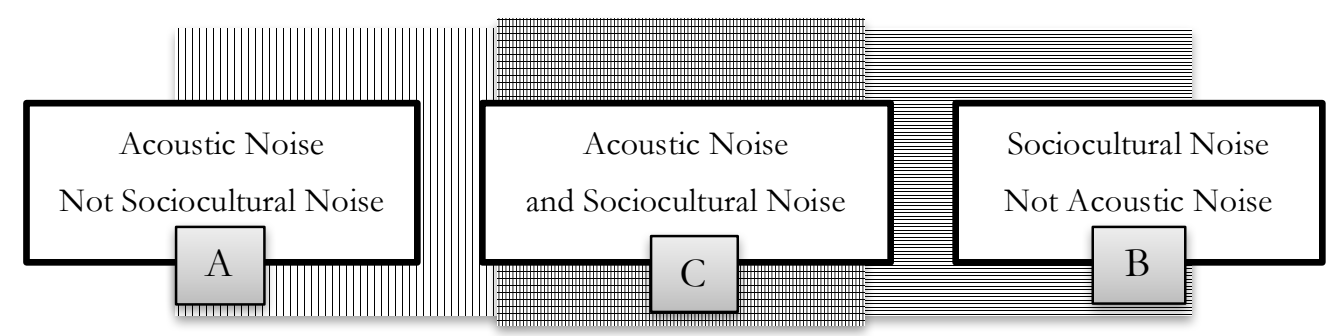

Figure 1-1. Different categories of noise.

\subsubsection{Redundant Information: Signal and Noise}

From an angle different than those introduced above, Canadian composer, scholar, and environmentalist R. Murray Schafer, in his Book of Noise, defines noise as "unwanted sound", anything that is "distinguished from signals" [9]. Information theorists Shannon and Weaver describe noise as anything that is extraneous to the transmitting message [10], while Michel Serres calls it background information [11]. This extraneous information in the background could be ignored, as Claire Taylor argues, by means of an “attention filter" [12]. However, it could also be used, as Kahn metaphorically points out via the example of a written scrawl which, although unfavourable to a teacher, could be a valuable source of information for a graphologist [13].

The usefulness of noise as "extraneous information" can be traced in both acoustic and sociocultural domains. The popularity of analogue electronic instruments in the digital era is an example where the "warmth" of the analogue signal, which is partially due to the presence of acoustic noise, is favourable over a "noiseless" digital signal. In 
the same vein, the extra aural information and background noise of everyday life can be seen as a valuable musical source via John Cage's idea of all-sound. According to Cage, "wherever we are, what we hear is mostly noise. When we ignore it, it disturbs us. When we listen to it, we find it fascinating" [3]. This is perhaps the cornerstone of the many works of contemporary noise and glitch music where extraneous information, whether acoustic or sociocultural noises (bursts of white noise, high-pitched sine tones, etc.) are reintroduced back into the signal, often using pulse-based and metric rhythmic patterns (such as the music of Carsten Nicolai aka Alva Noto).

\subsubsection{Pulse}

The presence of rhythm in music in all likelihood goes right back to the creation of the concept of music itself. As Pierre Boulez asserts, the concentration on pitch and rhythm in traditional musical notation clearly demonstrates their equally essential role as the basis of composition [14]. According to Aden Evens, Karlheinz Stockhausen goes even further in regarding rhythm as core to music by suggesting that "rhythm and pitches are in essence, the same things at different oscillations" [15]: an idea which was initiated in Henry Cowell's work of the early 1930s, specifically his Rhythmicon (one of the very first “drum machines") [16].

Alongside the evolution of certain trends in modern experimental music, where the pitch-based elements of harmony and melody lost the leading role they held in composition until the early $20^{\text {th }}$ century, rhythm became a central protagonist (most famously, through the challenging rhythms of Stravinsky's Le Sacre du printemps (1913)). A number of great examples of works in which rhythmic structure is the primary focus can be found in the works of experimental composer John Cage. According to Douglas Kahn, Cage "had no feel for harmony", and "he launched his contestation of western art music by placing rhythm over harmony" [13]. As discussed in the following section, the significance of rhythm can be observed more distinctly in an area of contemporary experimental electronic music that is explicitly concerned with noise, rather than what is conventionally perceived as musical. 


\subsubsection{Pulse-based Noise}

As Jacques Attali argues, the only thing shared between every kind of music is the principle of giving form to noise [17]. While modern technology makes it considerably easier to make music as "all that can be heard" and bridge the signal-noise schism, current electronic musicians can broaden the scope of musical perception by giving form to the rich resource of the "background" noise of our era. If parallel fifths do not sound wrong to our ears anymore, filtered white noise and granular synthesised percussive sounds, as well as the buzzes, clicks, and glitches of the new technologies, can also sound mellifluous if rendered familiar and realised in formally engaging ways.

With this in mind, metric and pulse-based rhythms can be seen as a framework through which marginalised "extra-musical" sounds can be brought back to musical life. In this process, the rhythmic grid takes the perceptually challenging noise of "irregular" sounds and makes it possible for them to be perceived as a signal through the "regularity" of pulse and metric rhythms. According to Claire Taylor, a "grid" makes it possible to control noise - that is, to give it form -, and "enhance the aesthetics of the message" [12]. She is speaking of visual noise, but in a musical transcription of her idea, a grid to give form to noise can be comprehended as pulse-based structures in metric rhythms. While for some, notably Trevor Wishart, the grid (or lattice in his parlance) unnecessarily limits sound, representing noise in a grid-based format of pulses and metric rhythms can empower their aural and musical effects. Creating hybrid musical forms using these polarities, that is noise (irregularity) and pulse (regularity) should not be seen as popularising the art and experimental music, but as an encouraging opportunity to "aestheticise" pop culture by opening more ears to new sonic experiences $^{3}$. Pulse-based metric rhythms can help the layperson's aural experience and appreciate the new sounds of experimental and art music. In this way, they can be utilised as Attali's decipherable code in the process of channelising and giving form to noise.

\footnotetext{
${ }^{3}$ Some examples of this can be found in works of IDM (Intelligent Dance Music) artists such as Aphex Twin and Autechre.
} 


\subsection{Objectives}

Taking a broad perspective, Jacques Attali describes music as "channelisation of noise" (which resonates with Varèse's well-known description of music as "organised sound"). According to Attali, "all music can be defined as noise given form according to a code (...) that is theoretically knowable by the listener" [17]. From both Cageian and Schaefferian viewpoints, it is the listener's role to take the effort in deciphering the code and thereby tune their ears to the "music" that exists in the noise. Attali's code can be also thought of as a semantic equivalent to what Landy refers to as "something to hold onto" in interpreting music [18]. In Landy's view, however, it is crucial for the composer organiser of sound - to take into account the need to help the audience decode the code. While exploration of new sonic ground has been core to the creation and reception of a vast number of works of organised sound, as Landy points out, a great deal of these works "[are] of marginal relevance to today's society in terms of its appreciation [of them]" [18]. Landy argues that this separation of art from life is also a consequence of "art for art's sake" doctrine. He suggests certain "access tools" that can help such works of sound art and experimental music - or in his terminology, "works of organised sound" - be appreciated by an audience broader than just our peer network of other musicians and sound artists. According to him, such access tools can equip the work with the "something to hold onto" factor, increasing its potential accessibility, and therefore the likelihood of its appreciation by a wider non-expert audience. With this in mind, the strategy of using metric and grid-based rhythms in regulating noise, as discussed above, can be thought of as a "hold onto" factor in giving accessibility to sounds that are normally perceived as unwanted, harsh, or uninteresting.

Landy also remarks on the strong effect of the visual aspect of sound-based works in enhancing accessibility, and claims that integration of visual accompaniments of some sort can help general audiences more easily connect to works of experimental music and sound art: 
It has been my experience that inexperienced listeners tend to find sound-based works more accessible when introduced in a convincing manner within audiovisual contexts regardless of what they are [18].

Accordingly, this research aims to build a framework in which rhythmic regulation and audiovisual expression are used as tools in aestheticising (or "musicalising", to adopt Douglas Kahn's term), and thereby boosting the accessibility and appreciation of noise. If music is "channelisation of noise", for the purpose of this research, to regulate the irregularity of noise through the regularity of a rhythmic grid is one important mode of channelisation. In also bringing back marginalised sounds to the aesthetic domain, audiovisual connectivity helps provide further such accessibility. Therefore, the overarching goal here is to investigate the design and construction of apparatuses that output hybrid characteristics: audiovisual systems that create sophistication out of simplicity (pulse), while regulating complexity (noise). With this in mind, and considering the different conceptual and contextual definitions of noise introduced in the previous section, this kind of hybrid system will create an audiovisual platform in which:

- At the acoustic level, Cowell's primary musical elements meet in the opposed states that is complex and irregular versus simple and metric: noise versus pulse,

- At the sociocultural level, conventionally extra-musical sonic territories are investigated and new sounds are recycled back into the domain of aural attention,

- At the information theory level, noise is reintroduced to the signal not as an extraneous parameter, but as an inherent and aesthetically valuable part of the message.

In order to achieve such a platform and turn theory into practice, appropriate tools and mechanisms are required. Above all, the system has to be able to:

- Create noise ${ }^{4}$,

- Regulate noise rhythmically and timbrally,

- Produce rhythms that are steady and pulsed,

${ }^{4}$ Sounds that are produced physically, but cannot be identified with a traditional instrument or a definite pitch. 
- Have audiovisual output.

Therefore, to meet these requirements and achieve the objectives set for this project, this research incorporates mechatronics for the following reasons:

- Mechatronic machines are noisy in essence,

- They are highly programmable and can be utilised as an efficient control system,

- They are capable of perfectly producing recurring motions with high accuracy,

- They are a physical system and can reflect the process of sound generation in a visible manner.

Accordingly, this research undertakes the design and construction of a system that employs mechatronics and microcontroller programming to give accessibility and appreciation to physically generated noise by structuring it on a rhythmic grid. A number of new mechatronic sound-sculptures ${ }^{5}$ will be designed, with rhythmically regulated noise as their sonic output. Even though this research is not concerned with conventional "musical" features such as pitch or melodic and harmonic relationships, the instruments will be divided into three different categories, defined according to different materials and mechanisms, in order to achieve a wide range of sonic outputs in terms of timbre and frequency. Therefore, the aim is to create an ensemble of different types of noise-intoners that includes high, mid, and low-range instruments that respectively cover different sonic territories. Upon completing the design and construction of these soundsculptures, they will be creatively employed in both performance and installation settings. In doing so, compositional approaches will be selected that share ideological, aesthetic, or structural ground with the ideas behind the design and development of the soundsculptures. Through construction of this system and its employment in some real-world compositional practices, the broader goal of this research is then to build a crossmedium frame of reference for an appropriate context in which the work of the author and those influenced it can be situated. In this way, the concept of "channelisation of

\footnotetext{
5 The term 'sound-sculpture' is used in order to highlight the emphasis on the physicality and visual aspect of the sound-generation.
} 
noise through a rhythmic grid" will be expanded and used in formalising a related set of contemporary practices and aesthetic trends in sound-based works of art that have to date received very little academic attention.

\subsection{Thesis Outline}

Figure 1-2 shows the structure of this thesis, each block representing one chapter. Chapter 2 provides a discussion on the background and related works that are organised in two categories: the use of noise and the use of mechatronics in sonic arts. The origins of both categories are examined, and considering the focus of this research, those areas concerned with pulse-based noise music and noise-oriented mechatronic sound art are more thoroughly surveyed. Chapter 3 presents a thorough discussion of the design, construction, and evaluation of three mechatronic sound-sculptures developed in this research, that is Rasper, Rippler, and Mutor.

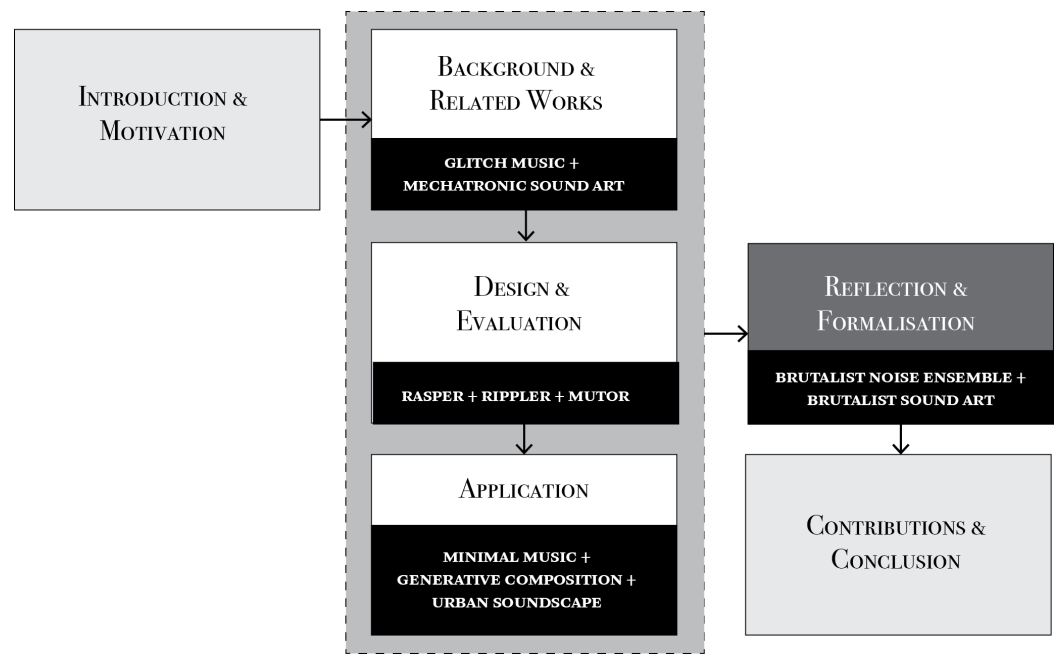

Figure 1-2. Thesis structure.

Different sonic and technical characteristics of the sound-sculptures are examined in detail, both individually and as a collective system. Chapter 4 is dedicated to the compositional application of the sound-sculptures and their utilisation in new approaches to working creatively with three established compositional practices 
(minimalistic composition, generative composition, and urban soundscape music). Chapter 5 establishes a new aesthetic framework inspired by Brutalism that formalises not only the work of the author, but also the broader context in which works presented in Chapter 2 can be situated. Coining 'sound-based brutalism' as a new aesthetic frame of reference for works that have not been examined in a comprehensive form within musical academia, this chapter also presents a series of works created with an explicit commitment to the realisation of a contemporary Brutalism through the medium of sound. Finally, Chapter 6 summarises the work that is presented throughout this document, reviews the novel contributions of this research, and lists the set of scholarly and artistic outputs that are derived from it. These contributions are briefly discussed in the following section.

\subsection{Summary of Contributions}

Using metric and grid-based rhythms, this thesis formalises a framework for aesthetic enhancement of mechanically produced extra-musical sounds. Through the design and development of an ensemble of new mechatronic sound-sculptures and employing them in three novel compositions, it extends the territory of glitch music into the physical realm. Coining the term "sound-based brutalism", this thesis solidifies a new aesthetic platform in appreciation of a large body of contemporary sound-based works that have been largely undervalued. In doing so, it expands the territory of Brutalism from visual arts to sonic arts.

A total of nine peer-reviewed publications, eight locally and internationally exhibited artworks, two awards, and numerous media features that are derived from this research, highlight its artistic and scholarly contributions to the field.

\section{Some of the contents of this chapter were featured in the following publication:}

Mo H. Zareei, Ajay Kapur, Dale A. Carnegie, "Noise on the Gird: Rhythmic Pulse in Experimental and Electronic Noise Music," In Proceedings of the 2013 International Computer Music Conference (ICMC), Perth, Australia (2013). 
12 


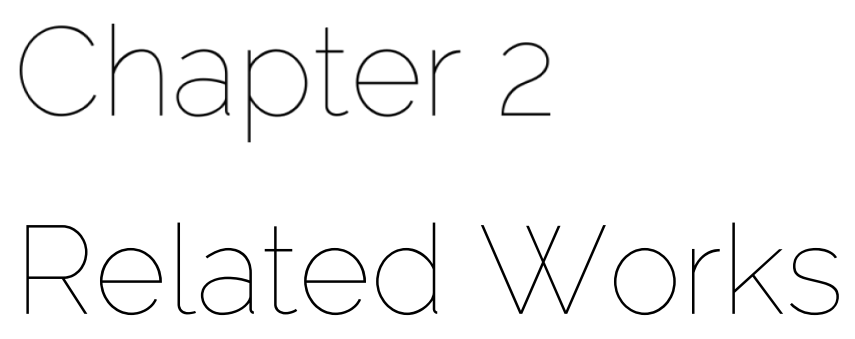

\subsection{Overview}

This chapter provides a number of significant examples of related works of experimental music and sound art that have, in one way or another, inspired and influenced this research. Based on the categories they belong to and the medium through which they are realised, these examples are discussed in two separate sections. Following a discussion on the background and history of the integration of extra-musical sound within music, some significant examples of noise music are presented, and the path leading to contemporary laptop-produced glitch music is briefly discussed. Considering the focus of this research, works in which metric and pulse-based rhythmic structures are prominent are highlighted and their primary characteristics reviewed.

The second half of this chapter is dedicated to works of sound art that incorporate robotic and mechatronic tools and technologies. After providing a historical overview on the integration of mechatronics and music, a new classification of works of mechatronic sound art is suggested. Based on this classification, this chapter then focuses on a selected number of those examples in which the use of conventionally non-musical sonic material is the key common feature.

The chapter concludes with a brief summary and paves the way for the methodology and implementation section of the research, starting in Chapter 3. 


\subsection{Noise on the Grid}

\subsubsection{Background}

At the onset of the $20^{\text {th }}$ century, the birth of extra-musical sounds as music was officially announced in Luigi Russolo's Futurist manifesto. Fascinated by the rich aural possibilities provided by the machines of the post-industrial era, Russolo invited musicians to "break out of the limited circle of sound" [2]. He pioneered this revolution by creating his orchestra of noise-intoners (Intonarumori) (see Figure 2-1).

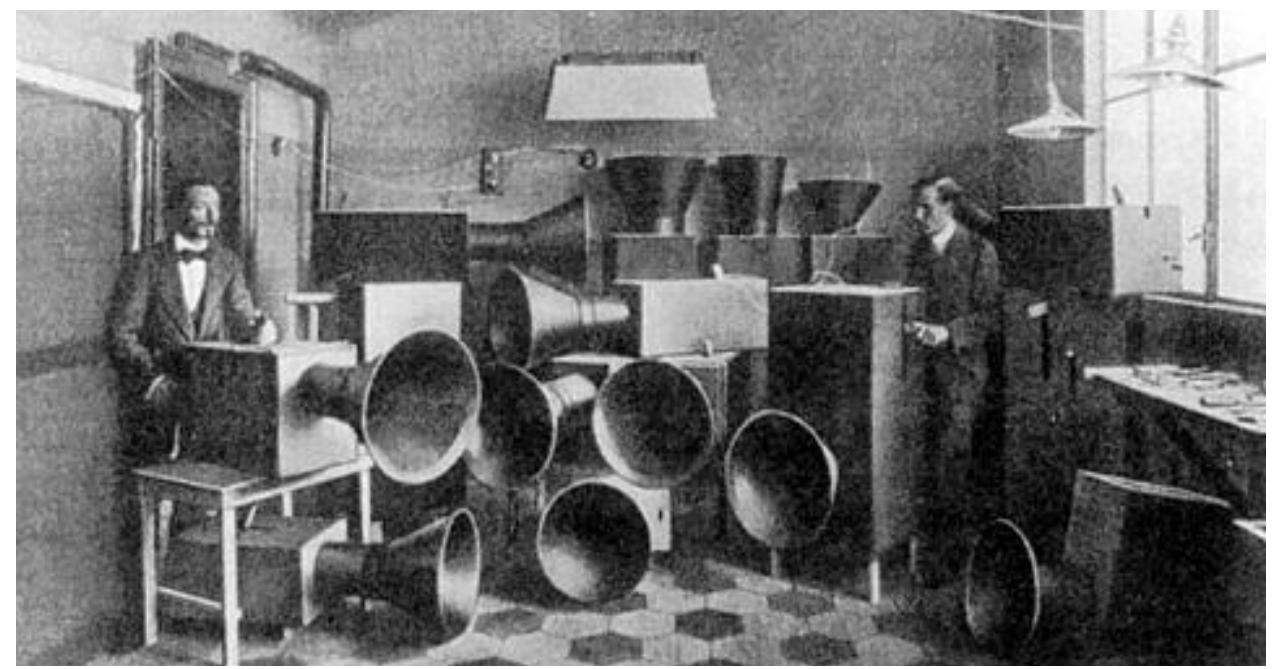

Figure 2-1. Luigi Russolo (left) and his Intonarumori. By Luigi Russolo, via Wikimedia Commons.

While Russolo's contribution was the incorporation of extra-musical sound in music, John Cage took this significantly further by "extending the process of incorporation" to everything potentially audible [13]. In other words, if Luigi Russolo and the Futurists "brought incidental noise to the foreground, John Cage would give permission to all composers to use any sound in composing music" [19]. Nevertheless, Cage himself credits Varèse as "having fathered noise" [13]. Varèse, who called music "organised sound", found "electronics" a new "liberating medium" that would help free sound from "the arbitrary, paralysing tempered system" [8]. 
With Pierre Schaeffer's Cinq études de bruits (Five Studies of Noise) (1949), concrete music $^{6}$ was born. Schaeffer's most important contribution, as Trevor Wishart notes, was to draw attention to the essence of sound itself, disregarding the source that produces it [7]. The phenomenological technique of 'ecouté réduit' (reduced listening) introduced by Schaeffer allowed a trained listener to listen to sound as sound and strip away the noise of causality and semantics. On the other hand, from the acoustic angle, the mutual persistence of sound and noise is later demonstrated in Stockhausen's music. As he "gradually removes the periodic elements of a sound to leave noise and then reintroduces regularity", Stockhausen "magnifies the boundaries of these temporalities to question their distinction" [15]. Kontakte (1958-60) is an example in which his aim is to "reconcile polarities (acoustic-electronic, pitch-noise, determinate-indeterminate, and so on) by creating a scale of intermediate states" [20].

This noise-sound continuum conceptualised by these pioneers prepared the ground for the following wave of experimental music. The exponential rate of development in music technology and the integration of electronics in music in the second half of the $20^{\text {th }}$ century opened up the opportunity for musicians to investigate new sonic environments through modern technologies.

\subsubsection{Contemporary Pulse-based Glitch}

Continuing throughout the $20^{\text {th }}$ century, the integration of noise and extramusical sound within music changed form corresponding to newly available technologies. In fact, Russolo's claim with regard to the connection between "the evolution of music" and "the multiplication of machines" [2], can be succinctly observed in the transformation of glitch music itself: from Christian Marclay's experiments with turntables, Yasunao Tone's damaged CDs and Nicolas Collins' modified CD players (Figure 2-2), to Carsten Nicolai's laptop-produced glitch. In all these instances, some undesired sonic byproducts of technology, as Hainge describes, are integrated "into an aesthetic construct, as primary content" [3].

\footnotetext{
6 musique concrète
} 


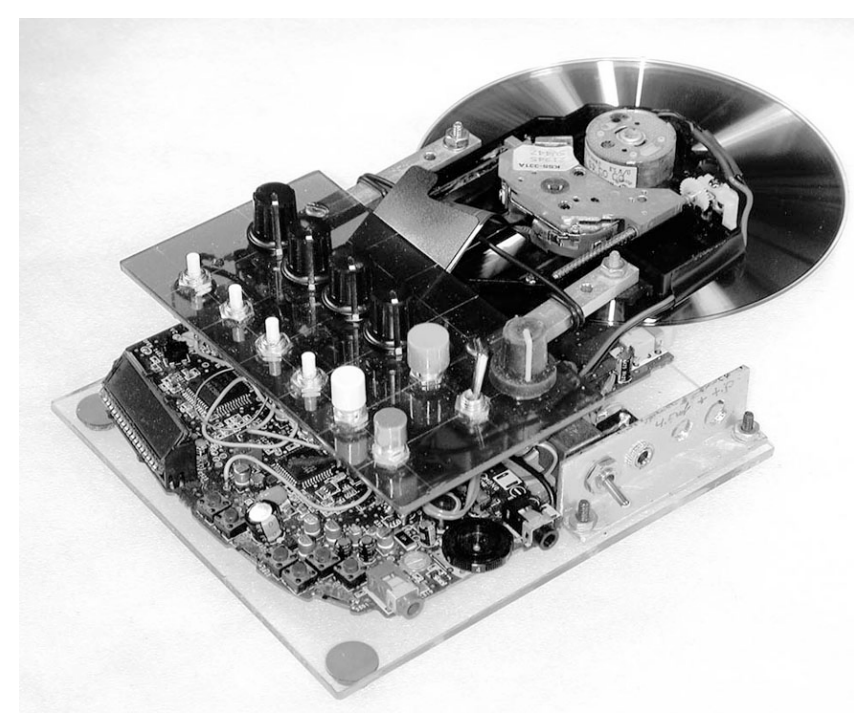

Figure 2-2. Nicolas Collins' Sled Dog: hand-scratchable hacked CD player. Photo by Simon Lonergan, courtesy of Nicolas Collins.

In his article in Organised Sound, Adam Collis states that the presence of noise in the music of the $20^{\text {th }}$ century, that started with "musicalising noise" (accepting the concept of noise as a valid musical material) and "noisification of music" (unconventional use of conventional instruments to create noisy sounds), was synthesised into a third state towards the end of the century in tandem with developments in technology: that is “using the sounds of the audio system itself" [20]. This whole process is expounded in Kim Cascone's article "Aesthetics of Failure" [19], and even more thoroughly in Caleb Kelly's book Cracked Media [21]. Collis categorises this creative approach into "lowering the signal-to-noise ratio" and "use of the errors, failures, ${ }^{7}$ and crashes of computer hardware and software” [20]. The former can be explained via Greg Hainge's discussion of noise in the context of communication theory, as he argues that not only is it impossible to fully eliminate noise from the signal, a certain amount of noise can be even semantically constructive for the communication [23]. In Hainge's words:

"What happens ... when the primary content of the sound processed by a highfidelity system is composed precisely of those sounds that the system is designed to eliminate?" [21]

${ }^{7}$ According to Hegarty, this failure "is not a judgment about badness, but one of a refusal of heroic success in the form of musical mastery or mastery of musical forms" [22]. 
These two methodologies became the cornerstone of the works of Oval and Pan Sonic duo, as well as those released under record labels such as Mille Plateaux, 12k, and Raster-Noton. This genre is often referred to as 'minimal glitch', a term that, according to Joanna Demers, can be interpreted as the non-academic version of microsound ${ }^{8}$ [24]. Torben Sangild notes that this music "focuses on sounds that are rarely recognized in everyday life; the forgotten secondary sounds of electronic equipment" [25]. In the vast majority of the works produced within this genre, metric rhythms and repetition are at the core of the composition. According to Hegarty, this is a music constructed of "digital debris", "errors", and "processing of normally extraneous sounds" that are re-organised into "beats" [22], or in the words of Philip Sherburne, "pops" and "clicks" that are "reconfigured into steady pulse of techno, with its unflagging 4/4 rhythm and almost polka-like backbeat" [26].

Loops and repetitions are certainly significant factors here. However, as Sherburne asserts - specifically about releases on the $12 \mathrm{k}$ record label - this loop "is not presented in the simple thud-thud-thud of the dance floor, but as a knotted code" [22]. This code helps the listener appreciate the essence of sound through repetition, as it exists independently of the source and the semantics, in accordance with Schaeffer's idea of reduced listening achieved through techniques such as the "locked groove". Hegarty argues that "beats are often there to be thwarted, either through complexity, or purposeful variation" [22]. In the music of Pan Sonic, as he describes it, noise residues layered on top of each other are brought back into musicality by steady beats and percussive elements that emerge in all layers. Of the music of Carsten Nicolai, he writes that beats "are overridden by hums, blasts, failings and direct glitch sounds" [22]. Nicolai's rhythmic beat patterns are described by Torben Sangild as "a gradual process for the listener" that "happens without being consciously noticed" [27] (see Figure 2-3).

\footnotetext{
${ }^{8}$ Microsound is a genre of computer music with a focus on sparse sonic structures that are constructed of series of short sound particles.
} 


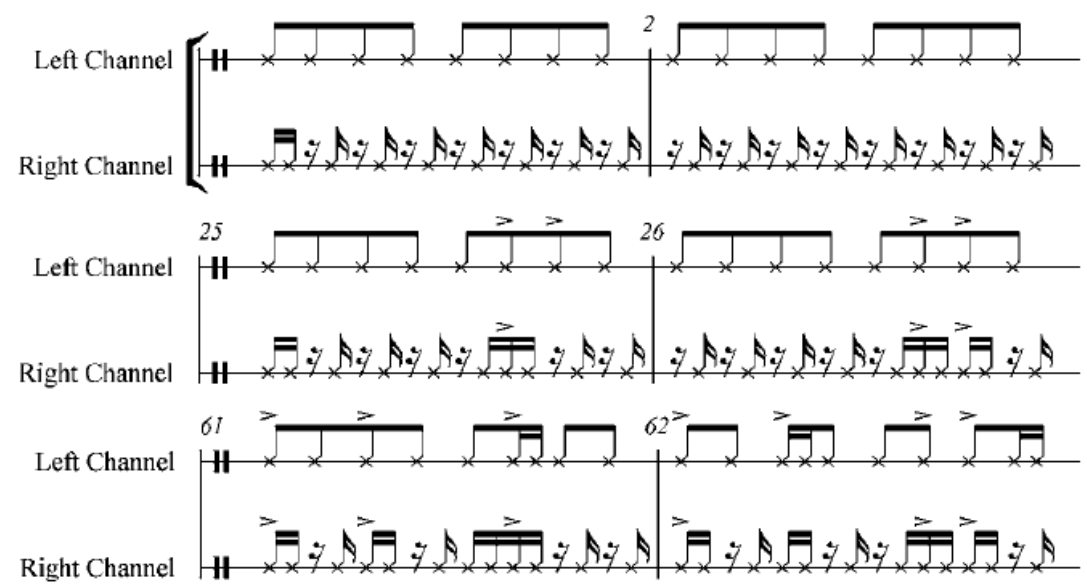

Figure 2-3. The development of a two-bar rhythm in Impulse by Carsten Nicolai, transcribed by Adam Collis [20]. Here, the sound material (noise, clicks, and sine tones) are articulated using metric rhythmic patterns.

In spite of these claims of varying degrees of rhythmic complexity, the vivid presence of pulse-based metric rhythms, in addition to the fact that most of these musicians are neither from "academic backgrounds in electroacoustic music" nor from the "commercial dance music scene" [20], identifies this music as a hybrid cultural form created through mixing polarities: high art and popular, academia and dance, noise and beat. Collis compares Nicolai's work with Stockhausen's, arguing that they are somehow similarly aimed towards reconciling polarities [20]; the reconciliation of irregularity and regularity which takes place on the noise-sound continuum. "Bridging the gap" between the academic and the non-academic is also a matter of concern for Cascone. He argues that association with "dance music" has deprived most of the work in this area from the "academic consideration and acceptability that it might otherwise earn" [20].

\subsubsection{Discussion}

As Joanna Demers states, "tonality, catchy melodies and rhythms, and sentimental themes" make music easily consumable [24], paving the ground for its commodification and mass-production: concepts that cheapen music for Attali. But what happens when tone and tonality are substituted with noise and atonality, catchy melodies are replaced with timbral manoeuvres and the sentimental theme is gone? Would the mere existence of a rhythmic grid still degrade the music? In his article in Computer Music Journal, Ben 
Neill critically argues that what makes a rift between "serious" and "vernacular" music is the "absence or presence of repetitive beat" [28]. Persistence of beat in the dance music played in clubs where music is - as Neill quotes Chadabe - "appreciated more in physical than intellectual terms" [29], is definitely a determinant catalyst in the creation of such notions. But is this argument any more valid than one suggesting all atonal music is dark and scary, because it is very often experienced in contexts of horror movies? Does prolific use of the minor key in sentimental tunes make the key itself sad in essence? As Neill argues, post-modernist thought provided the art-music composers of the 1980's a permit to employ elements of pop culture. Is there a reason not to extend this permit to the use of pulse-based rhythms and beats? In fact, as Neill argues, they can be simply envisioned as a platform (like the sonata form in classical music) through which electronic musicians frame their various compositional ideas [28]. Of course, the sole employment of such platforms does not guarantee success in achieving one's artistic objectives, nor in making the work more accessible. However, depending on the context and the manner in which they are used, grid-based rhythmic structures can be regarded as potentially powerful and valuable compositional and aesthetic tools (as in the examples discussed in 2.2.2).

In order for the modern noise-music to thrive, Cascone argues that "new tools must be built" [19]: new technologies can lead to new sounds, and these new sounds can be made more appreciable once expressed through some 'hold onto factors'. Therefore, tools that equip musicians with such abilities would assist them in further formalisation of the "infinite variety of noise-sounds", refining extraneous sonic information in order to build new sonic grounds.

"Pulse equals life, equals pleasure."

- Ben Neill 


\subsection{Mechatronic Sound Art}

\subsubsection{Overview}

As mentioned in the previous section, Luigi Russolo predicted that the evolution of music would occur in parallel with the multiplication of machines. An example for the validity of this claim can be observed in the integration of robotic techniques, mechatronic machines, and automatic apparatuses in design and construction of new musical tools and instruments. From a conceptual perspective, it can be suggested that there have been two different approaches towards this amalgamation. The first one is an effort to replace the human performer with machines in order to explore the full potential and push the boundaries of conventional musical instruments, while the other tries to investigate the machine itself not only as a performer, but also as a source of new sounds. In other words, in the first scenario, electromechanical machines have been used to create augmented musical instruments with extended performative capabilities, and in the other, they are used as extra-musical sound-objects utilised in a musical context. Although there is no rigid distinction between the two approaches and the dividing line here is blurry, in the context of this research, such classifications help clarify the author's motivation in highlighting certain works and artists in this section. Therefore, while conceding that the terminology is somewhat indistinct, the two strands it represents are useful in clarifying differences between 'musical robotics' and 'mechatronic soundobjects'. In the following sections, a brief overview of musical robotics will be presented, and the principles by which the above classification is supported are explained. Considering that the focus of this research is to pursue a means of creating sounds that are conventionally perceived as "extra-musical", mechatronic sound-objects are then discussed in more detail.

\subsubsection{Musical Robotics}

Semantically, musical robotics is perhaps best explained via 'automatophonics', a term used by Charles Fowler to describe "mechanisms that replace the human performer, but not the instrument itself' [30]. According to Fowler, the production of these types of 
mechanical musical instruments - which are as old as the water organ built in 875 C.E. reached its climax after the Industrial Revolution, the player piano being the most significant instrument within this domain. As Murphy argues, since these early generations of automatic musical instruments were primarily built as tools for the reproduction of music, they gradually died out in the early $20^{\text {th }}$ century with the advent of the phonograph [31]. However, during what Murphy calls "the 1970s Renaissance", a new movement, which over the past decade has been led by figures such as Eric Singer, Gil Weinberg, and Ajay Kapur, was revitalised (Figure 2-4).

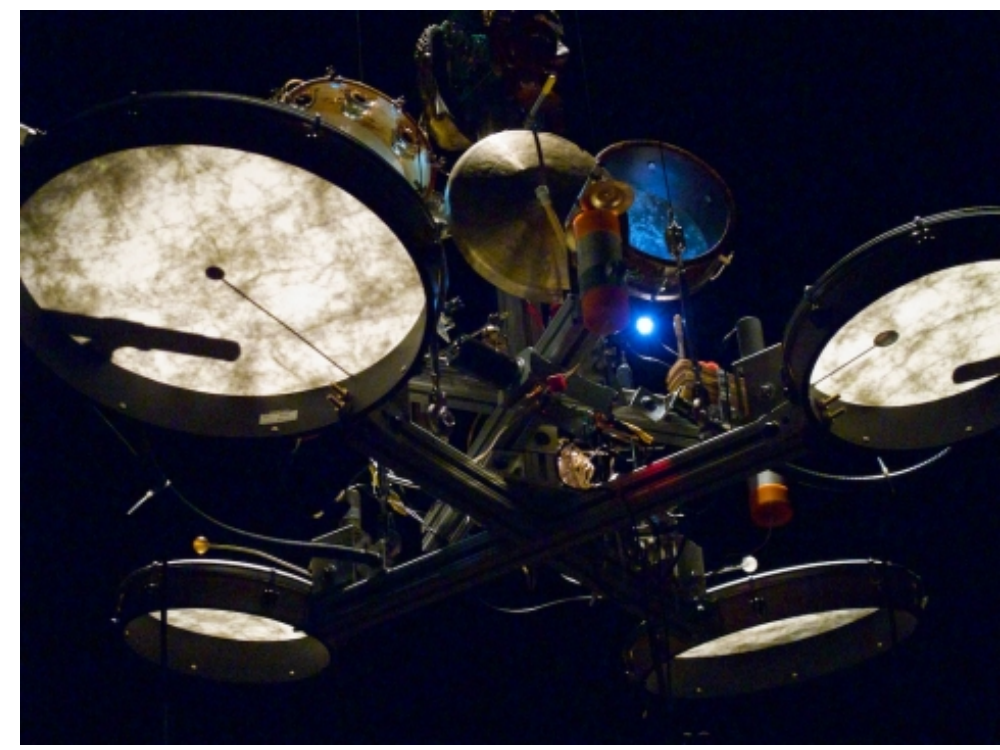

Figure 2-4. Kapur's MahaDeviBot. Photo by Jim Murphy, courtesy of Ajay Kapur.

According to Murphy, this movement was an effort to create "a reality consisting of real-world production of sounds, forgoing the loudspeaker in favor of mechatronically facilitated actuation techniques capable of truly localized sound" [31]. For instance, the musical robotic works of Eric Singer, founder of the League of Musical Urban Robotics (LEMUR), "focus on augmented instruments and new instruments inspired by existing designs" [31]. As Murphy describes, among LEMUR's numerous musical robotic projects [32], Singer's "Guitarbot" [33] is one of the most significant examples in the field. Other examples can be found in Weinberg's work. In his article "Towards Robotic Musicianship", he explains in detail the design and construction of his robotic percussionist "Haile" from a musicianship perspective [34]. In addition, the KarmetiK 
Machine Orchestra - an ensemble of laptop performers and a set of networked robotic idiophones and membranophones designed by Ajay Kapur, Michael Darling et al. [35] is another example of robotic instruments that are capable of performance in areas (such as speed and precision) beyond human performers' abilities.

In addition to the significant examples above, Phil Dadson's instruments ${ }^{9}$ (created in the 1980s), Jim Murphy's recent works on robotic guitars [36], and many works described in Kapur's article “A History of Robotic Musical Instruments” [37] are among numerous other examples of work in the musical robotics field; one that that was certainly inspired by groundbreaking works of Godfried-Willem Raes (Figure 2-5) and Trimpin. The diverse contributions of Raes and Trimpin - from musical robotics and electroacoustic instruments to kinetic sound-sculptures and mechatronic sound-objects are also responsible for blurring the boundary line between musical robotics and mechatronic sound-objects.

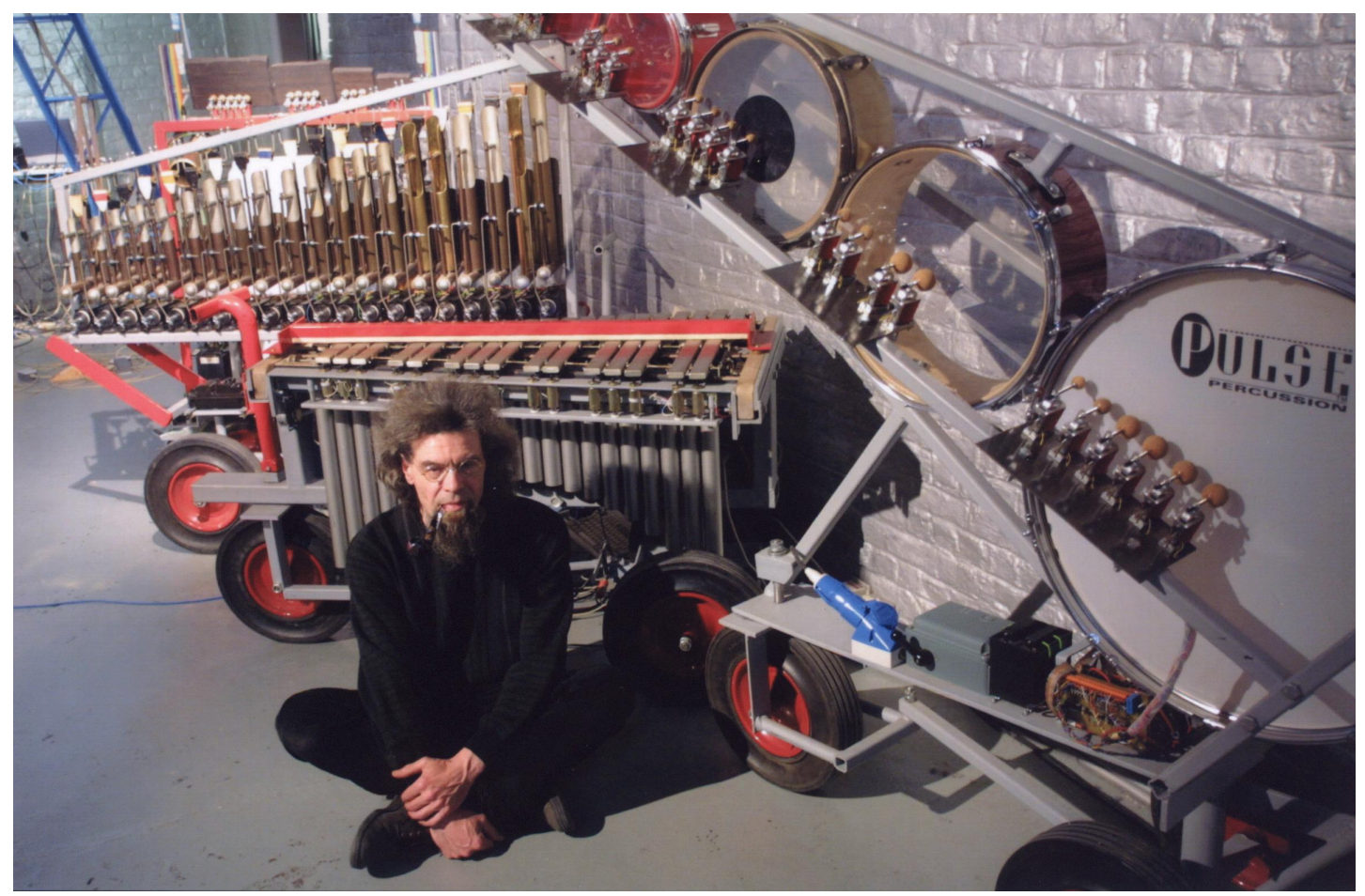

Figure 2-5. Godfried-Willem Raes and his mechatronic musical instruments. Photo from www.logosfoundation.org, courtesy of Godfried-Willem Raes.

\footnotetext{
${ }^{9}$ See "From Scratch" archive at www.sonicsfromscratch.co.nz (Retrieved on September 2, 2013).
} 


\subsubsection{A New Classification}

From the early days of the hybridisation of music and robotics, electromechanical devices such as linear actuators, servos, and DC motors, have been the basic key components in the creation of new instruments, musical systems, and works of kinetic sound art. However, regardless of their popularity and numerous positive features such as high durability, extensive availability, relatively cheap cost, and the fact that they are fairly easy to work with, one major issue, which many works of musical robotics are challenged by, is the relatively loud "noise" of these components. As mentioned in the previous section, the integration of robotics in the musical world, in many cases, has been geared towards creating instruments that are inspired by already-existing musical instruments, with automated and/or augmented performance capabilities. In this approach, motors and actuators are popular tools, used to create the various motions that generate the automatic actions such as beating, stroking, plucking, etc. In other words, motors and actuators are used as a means to create the mechanical force, which then leads to the sound-production, and not as the source of sound itself. Therefore, in such systems, the noise produced by these electromechanical devices is often musically extraneous, and an unwanted by-product of the sound-production mechanism. Accordingly, finding a workaround to overcome this issue can be a matter of concern in developing such musical robotic systems. For instance, in Kritaanjali, a robotic harmonium that uses a solenoid-based actuation method, even though $24 \mathrm{~V}$ solenoids have been used to press the harmonium keys, "the minimum voltage possible to press each key was applied" [38] in order to prevent the actuation noise caused by the solenoids.

Creating automated and augmented versions of already-existing traditional musical instruments is not, however, the only path taken by the investigators of music technology, sound art, and robotics. There are, in fact, a great number of works of robotic and mechatronic sound art which explore "extra-musical" noise rather than musical sound. Contrary to the 'musical robotics' trend, in these works, which are identified here as 'mechatronic sound-objects', the sonic resemblance to what is expected from already-existing musical instruments is replaced by a focus on the conventionally 
perceived "extra-musical" sonic territories provided by the machines and technologies of the new era. To summarise, the difference between the categories of musical robotics and mechatronic sound-objects can be explained as follows: in the first group, mechatronic components are used to actuate a - conventionally understood - musical object (such as guitar string, drum skin, etc.), while in the second group, they are used to actuate a non-musical object.

\subsubsection{Mechatronic Sound-objects}

The roots of the mechatronic sound-object trend originate in Luigi Russolo's fascination with the post-Industrial Revolution noisescape and his Intonarumori [2]. Here, preserving the integrity and purity of a certain musical sound that is expected from a musical instrument - either melodic or percussive - is not a concern, and in fact, the goal is to investigate non-musical noises and tones. Therefore, the sonic by-product of the components that comprise a mechatronic system would not be considered as compromising to these works of mechatronic sound art.

It is reasonable to regard Luigi Russolo's Art of Noises as the conceptual cornerstone of the mechatronic sound-objects trend. As discussed in the previous chapter, in his Futurist manifesto, Russolo calls for artistic investigation of the noises of the machines in order to expand the "limited variety of timbre" provided by the orchestra at the time. As first instances of the conventionally "extra-musical" sounds of the machine integrated in a musical setting, his Intonarumori continue to influence the artists and musicians in the realm of experimental and art music to this day. Accordingly, one of the main precursors of the contemporary mechatronic sound art and the founder of the Logos Foundation, Godfried-Willem Raes, openly expresses his fascination for Russolo's instruments [39]. Influenced by the anti-authoritarianism of the day, Logos was founded in the late 1960s by Raes and a number of other artists and instrument designers. Raes' goal was to defy what he regarded as the authoritarianism of music-production industries, by pushing the boundaries of music and sound art through design and construction of new instruments and integration of these instruments and electromechanical technologies in his various works of sound art [39]. 
Another key figure, whose contributions to the field of mechatronic sound art, according to Murphy, "either directly or indirectly, [...] significantly influence the majority of subsequent work in the field", is Trimpin [31]. Murphy describes Trimpin's work as a full "rejection of the loudspeaker through the use of physical objects actuated mechatronically and placed throughout an installation space"[31]. A selected number of Trimpin's sound art works are presented in Trimpin: Contraptions for Art and Sound, complied and edited by Anne Focke [40].

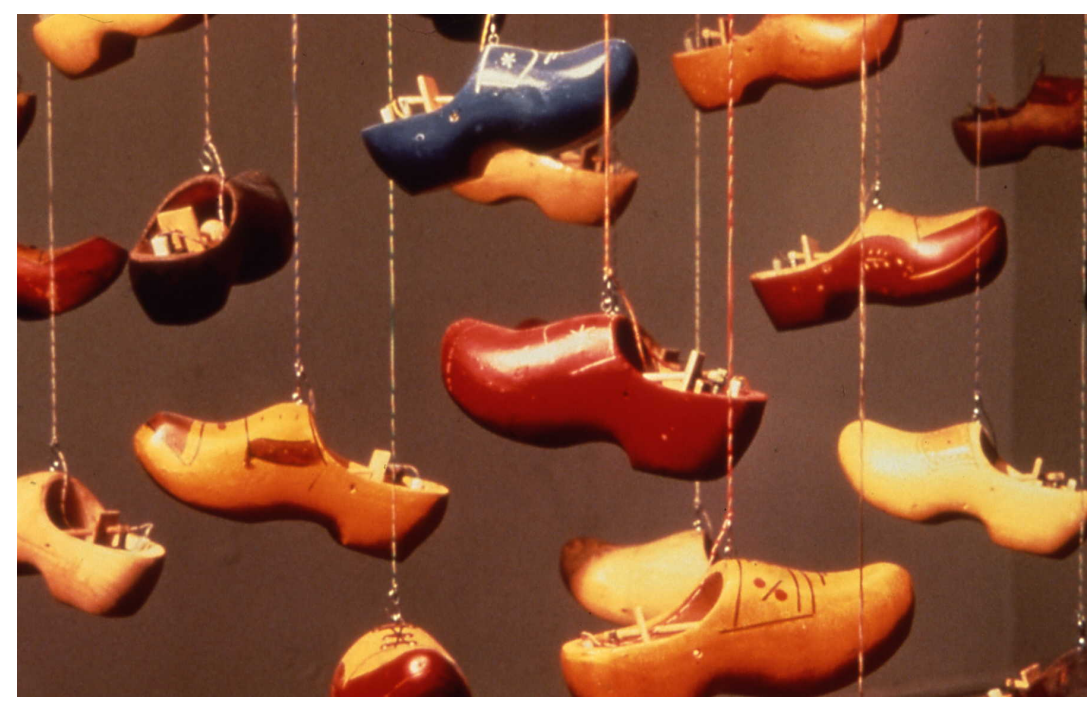

Figure 2-6. Klompen by Trimpin (1990): electronically driven mallets placed in wooden clogs. Photo courtesy of Frye Art Museum and the artist.

One of the main principles in Trimpin's work has been the sonic recycling of found objects and obsolete machines through mechatronics in order to create kinetic soundsculptures (see Figure 2-6). ${ }^{10}$ This practice can be traced through works such as Gordon Monahan's Multiple Machine Matrix in addition to a number of others cited in Murphy's article [31]. Multiple Machine Matrix is a "multi-functional performance and installation environment of automated machine sculptures built from electronic surplus and trash [in which] MIDI signals [...] control the movement of mechanical/robotic devices such as voltage-modulated steel sheets of various sizes, pulse-controlled bi-directional metal sheet Doppler spinners, and percussion-activated furniture” [42]. Peter Garland writes of

\footnotetext{
${ }^{10}$ In fact, as Jean Strouse writes, "[Trimpin] moved to the United States largely because Americans throw out a lot of more of the high-tech junk he uses in his work than Europeans do" [41].
} 
this work that it is the sound that is "the source of amazement and pleasure", and gives life to the machine, not vice versa [43]. In addition to works of Raes, Trimpin, and Monahan, the Fluxus machines of Joe Jones, and creations of Jean Tinguely, Martin Riches, and a number of others discussed in Alan Licht's Sound Art [44], are other examples that contributed to the development of the mechatronic sound-object trend.

\subsubsection{The New Generation}

Contemporary examples of sound art combining non-musical objects with mechatronic machines can be found in work of the Canadian artists Nicolas Bernier and Martin Messier. As a direct homage to Russolo's instruments, Bernier and Messier's La chambre des machines (2010) is a project in which "machines made of gears and cranks are manipulated to produce a sound construction at the crossroads of acoustics and electronics" [45]. The project is in fact a live act consisting of the duo and their mechanical noise instruments where "each performer approaches his apparatus like a technician" (see Figure 2-7) [46].

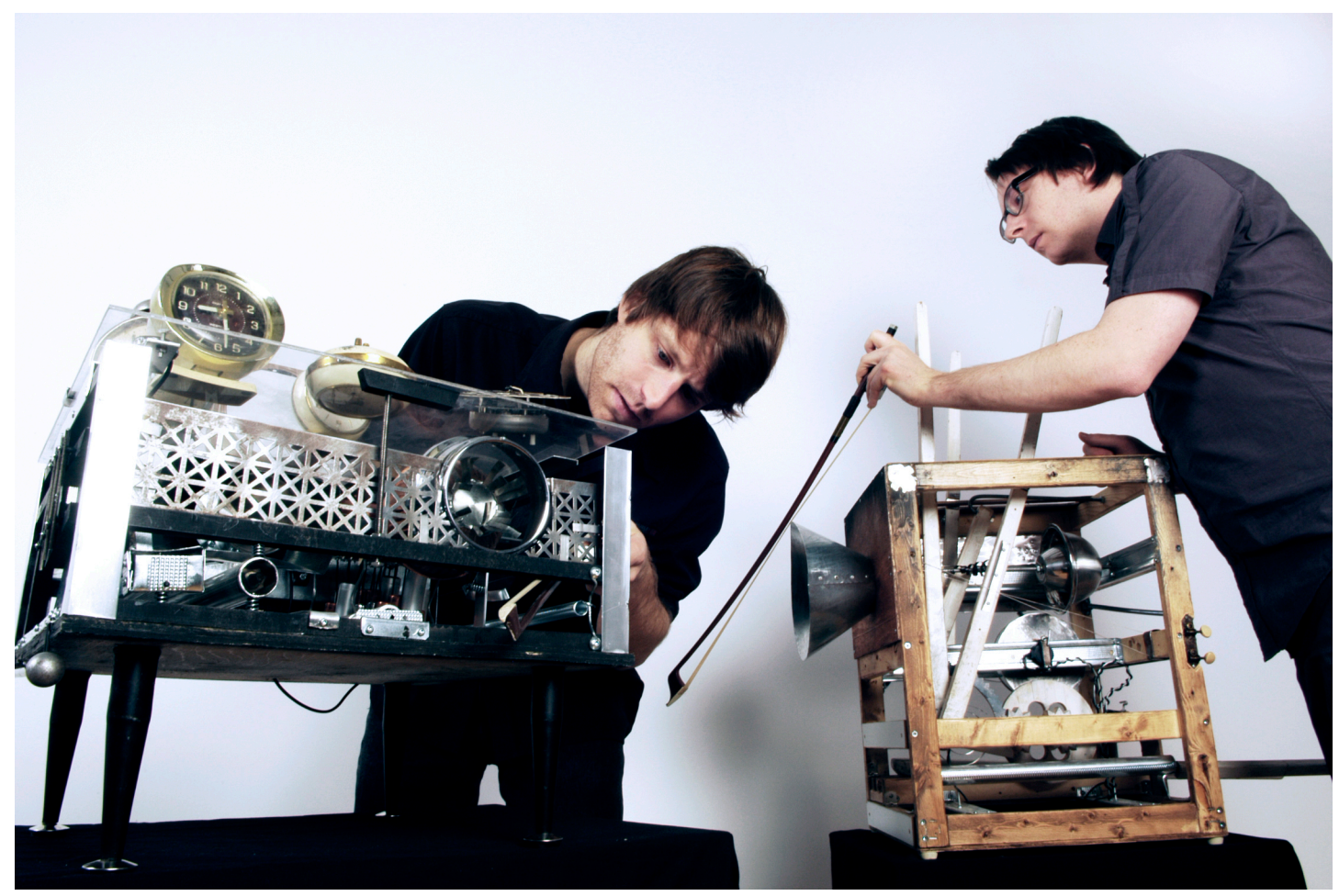

Figure 2-7. La chambre des machine by Nicolas Bernier and Martin Messier. Photo courtesy of the artists. 
Both instruments are of course inspired by Russolo's Intonarumori, and although they generate sound both physically and mechanically, the exclusive use of autonomous mechatronic machines is not investigated here as it is in the artists' solo projects. Bernier's award-winning Frequencies (a) (2012) “is a sound performance combining the sound of mechanically triggered tuning forks with pure digital sound waves. The performer is triggering sequences from the computer, activating solenoids that hit the tuning forks with high precision. Streams of light burst in synchronicity with the forks, creating a not-quite-minimal sound and light composition" [47] (Figure 2-8 Left).

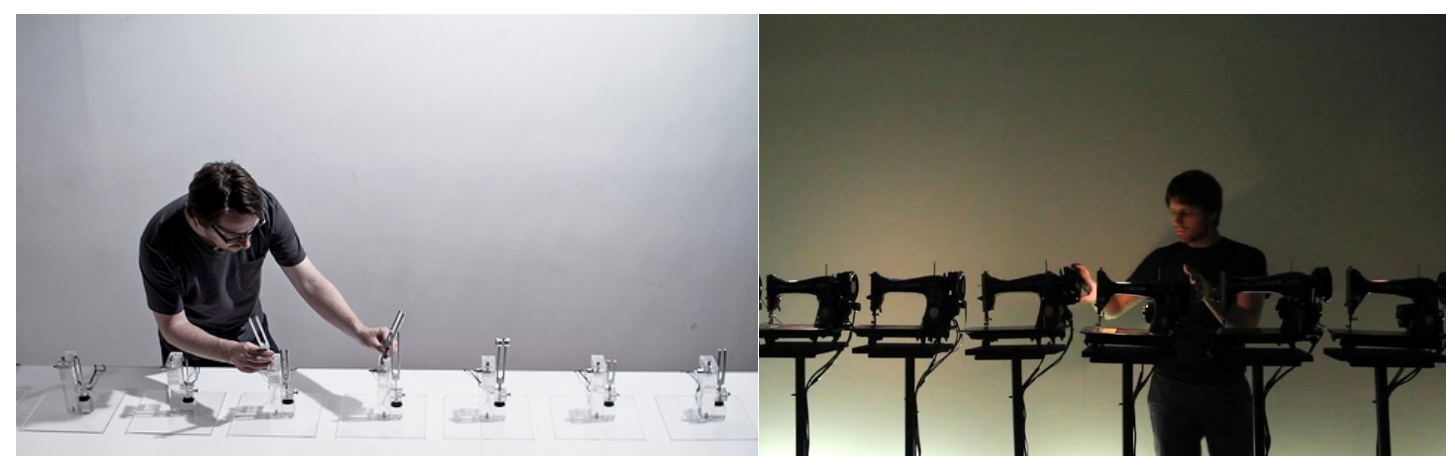

Figure 2-8. Left: Bernier's Frequencies (a) (2012), photo courtesy of the artist. Right: Messier's Sewing Machine Orchestra (2010), photo courtesy of the artist.

Similarly, Messier's Sewing Machine Orchestra (2010) is an audiovisual performance in which "computer processing transforms the functional sounds of eight 1940s Singer sewing machines, mounted on stands, into a vivid, dancing weave of hums, whirrs, and beats, accompanied by suitably pulsating lights" [46] (Figure 2-8 Right). The emphasis on the audiovisual expressivity and visual aesthetics in such works is simplified in Fowler's explanation: "many of these mechanical instruments are clever gadgets and novelties jewel boxes and the like - intended for looks as much as for sound" [30].

Remarkable examples of using mechatronics as the source of sound itself can be found in works by the Swiss artists Zimoun and Pe Lang. A significant majority of their solo works, as well as those that they create in collaboration with each other, are largescale sound installations that comprise significant numbers of what they refer to as “prepared DC motors or actuators" as sound-objects (see Figure 2-9). 
According to Murphy, “Zimoun's and Lang's works have much in common with those of other artists who create their own instruments: many such works involve reductionist sculptures that pare sound-making elements down to their pure forms" [31]. Ethan Rose compares “the repeating forms of single points of sound” in Zimoun's work to the 'cut-copy-paste' of contemporary digital music, and argues that "Zimoun has successfully translated this digital editing process into a mechanical, physical representation" [48]. By simultaneous sonification of a large number of sound-objects (that is small actuators attached to cardboard boxes, wires, cotton balls, etc.), Zimoun and Pe Lang immerse the space in a deep sonic sea that gently moves through pulsating timbral waves.

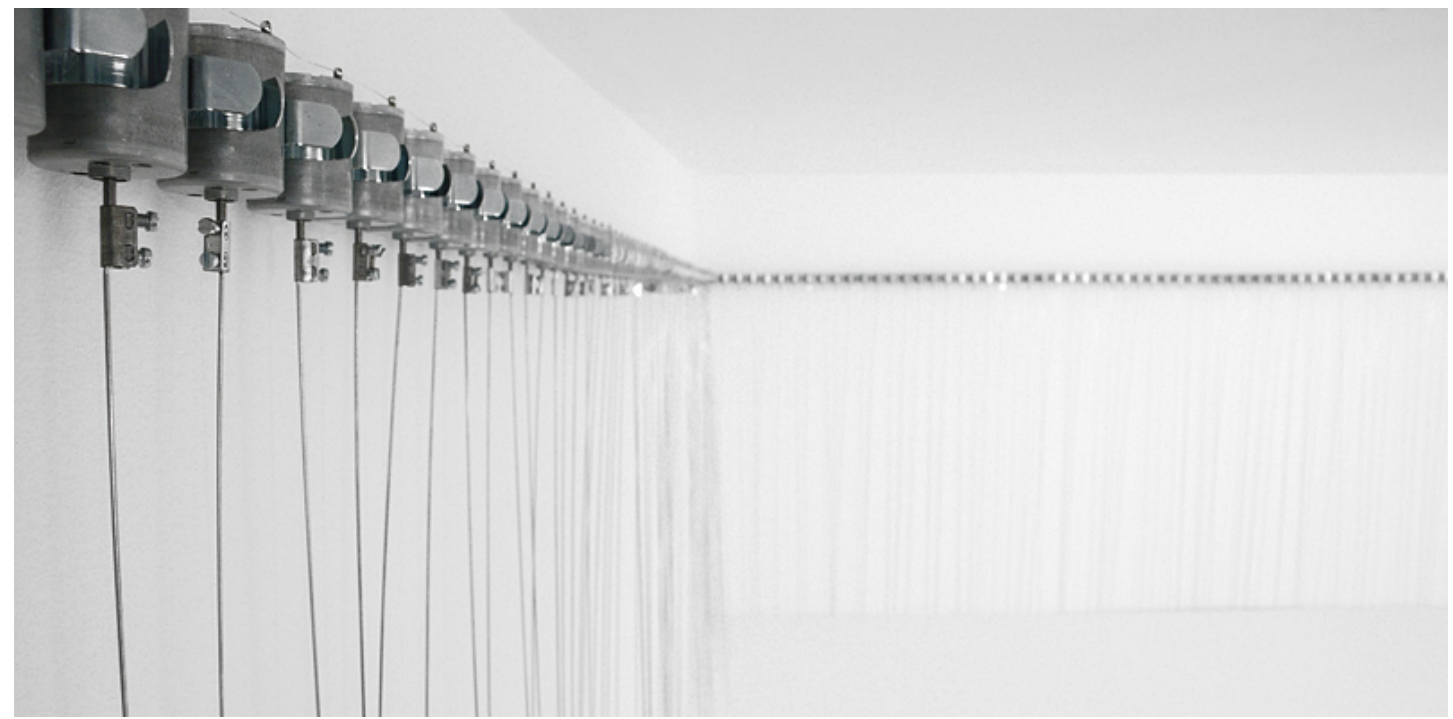

Figure 2-9.216 prepared DC motors, filler wire $1.0 \mathrm{~mm}(2009 / 2010)$, by Zimoun. Photo courtesy of the artist.

Other contemporary examples of using non-musical electromechanical tools and objects to create audiovisual works can be found in Murphy's Metal+Motors (2011), Daito Manabe's Motor Music Test (2013), and The Enlightenment (2014) by Quiet Ensemble. Metal+Motors is an installation "consisting of a variety of DC motor actuators striking metallic objects in response to ultrasonic sensor data" [49] that are derived from audience's movements in the gallery space. 
Similarly, Manabe's Motor Music Test is an audiovisual work comprising an array of metal sheets, each attached to a heavy-duty servomotor (see Figure 2-10). The servomotors are programmed to rotate the metal plates at various speeds and directions, within various degrees and ranges, creating variation of rhythmic phrases from a combination of the sound of spinning sheets and the buzzing of the motors.

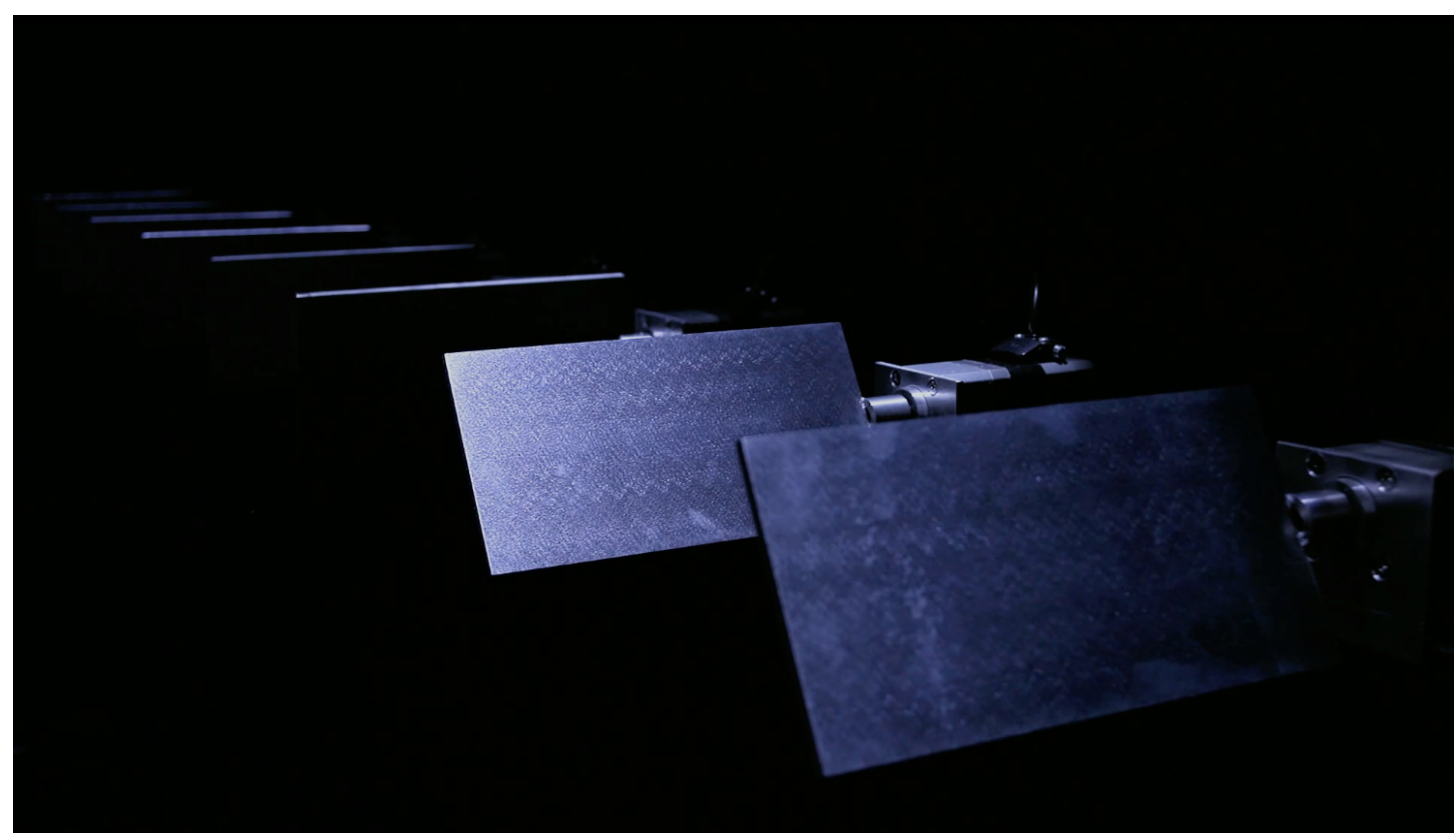

Figure 2-10. Motor Music Test by Daito Manabe. Photo courtesy of the artist.

Lastly, in The Enlightenment the Italian duo Fabio Di Salvo and Bernardo Vercelli "have taken the soft hum of electricity and turned it into a booming orchestra of sound and light ...[that] is made from 96 lamps - neon tubes, spotlights, theatre rigs and strobes - all of which produce their own sound" [50].

\subsubsection{Discussion}

Even if we do draw a line to conceptually differentiate between the 'mechatronic soundobject' trend that is rooted in Russolo's The Art of Noises, and the one devoted to the development of robotically augmented conventional musical instruments, there still exists a strong connection between the two. After all, it cannot be denied that many of the technical developments of the 'mechatronic sound-objects' trend are inspired by the 
intensive research and engineering that has been done on the works that, in the above classification, fit among the 'musical robotics' trend.

"With machines, life has created the most immense, the most varied sources of noise".

- Luigi Russolo

\subsection{Summary}

This chapter has provided an overview of the two major disciplines of experimental music and sound art that have influenced the formation of the core ideas underlying this research, along with a number of significant illustrative examples.

The embodiment of noise and the employment of mechatronics in music and sound art were assessed separately, and a new classification in characterising the works of mechatronic sound art was introduced. A select number of works influential to the evolution of contemporary digital glitch music and mechatronic sound art were discussed. Although realised through different mediums (digital versus physical), the examples of noise music and mechatronic sound art mentioned here share one pivotal feature: they all base their primary sonic material on sounds that are conventionally perceived as extra-musical. It is this common ground that forms the basis for the development of this thesis. Building upon this shared material, this research project aims to achieve the hybridisation of the two disciplines by choosing its medium from one (physical/mechanical), and sculpting it using structural principles used in the other (grid/metric rhythm).

The next chapter presents a series of mechatronic sound-sculptures designed and developed over the course of this research, which build a creative platform for "channelisation of noise" and the realisation of the objectives discussed in the previous chapter. 
The research presented in this chapter was featured in the following publications:

Mo H. Zareei, Ajay Kapur, Dale A. Carnegie, "Noise on the Gird: Rhythmic Pulse in Experimental and Electronic Noise Music," In Proceedings of the 2013 International Computer Music Conference (ICMC), Perth, Australia (2013).

Mo H. Zareei, Ajay Kapur, Dale A. Carnegie. "Rasper: a Mechatronic Noise-intoner," In Proceedings of the 2014 International Conference on New Interfaces for Musical Expression (NIME), London, UK (2014). 
32 


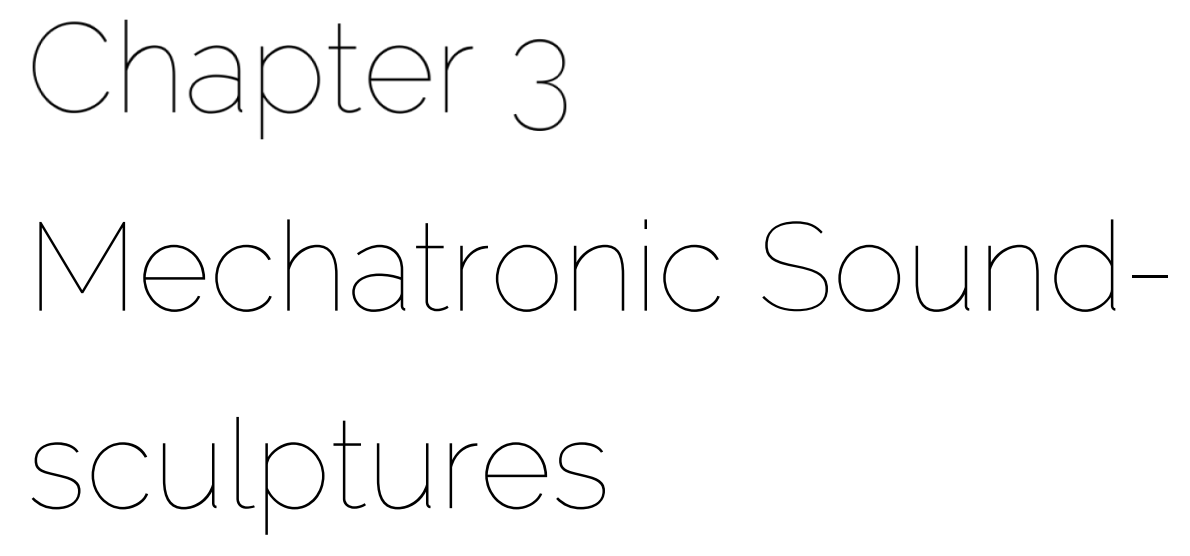

\subsection{Overview}

Inspired by the contemporary examples of glitch music and mechatronic sound-objects presented in the previous chapter, this research looks at creating a framework in which these two areas of contemporary sound art are fused and hybridised (Figure 3-1). In pursuing this goal, a series of new mechatronic sound-sculptures are designed and developed, merging key elements from contemporary glitch music and mechatronic sound-objects.

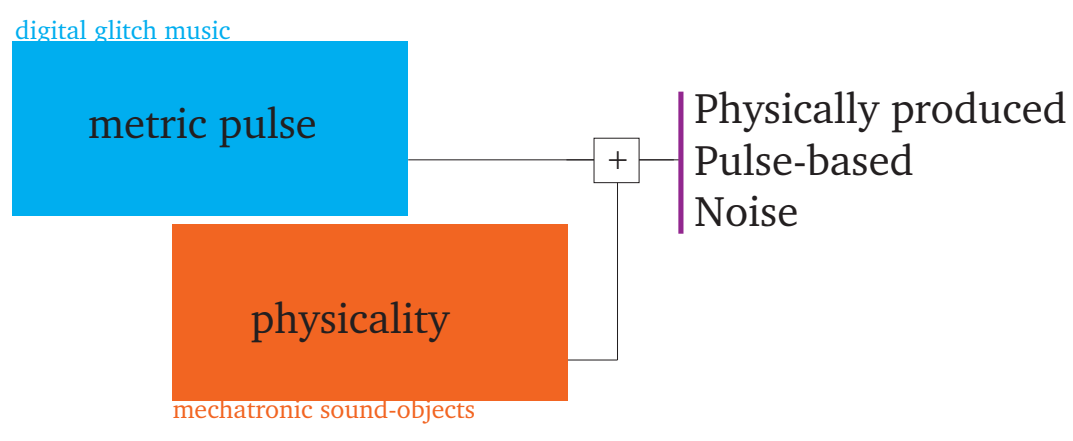

Figure 3-1. Hybridisation of contemporary digital glitch music and mechatronic sound-objects by combining some of their key elements. 
The series comprises of three different instrument-types that are all created with the objective of aestheticising physically generated noise through rhythmic and timbral regulation, in an audiovisual manner. While sharing the key ideological and aesthetic cornerstones, each type of sound-sculpture uses a different functional mechanism, and its sonic output contributes to a different frequency and timbral range. The three mechatronic sound-sculptures are entitled Rasper, Rippler, and Mutor, inspired by the way in which Russolo named his noise-intoners based on their style of noise-generation. Combined, these sound-sculptures form an aesthetically coherent system that is equipped with a range of different rhythmic and timbral qualities.

Throughout the development of the sound-sculpture series, a number of principles that are derived from the thesis objectives have informed the design process. As discussed in the first chapter, the overarching aim of this thesis is to explore the artistic potential of aesthetically devalued technological artefacts not only sonically, but also visually. Therefore, we endeavour to enable this exploration by using widely available basic mechatronic components and non-musical objects as the sound-sculptures' raw material, and to emphasise their normally ignored physical appearance. Considering this strong focus on materials and components, the key design decisions throughout the entire process are driven by a concern for clarity, simplicity, and functionality (for further discussion of these qualities see Chapter 5). Accordingly, in all three sound-sculptures, the components are held together in simple and minimalist transparent enclosures. In this way, while serving a primarily functional purpose, the enclosures also facilitate the full exposure of the entire sound-generating mechanism. Considering the emphasis on the audiovisual mode of expression, to further highlight the visual aspect of the work and to boost the audiovisual connectivity, each instrument also employs a light element. The aesthetic decisions influencing the choice of light are explained in 3.2.2.4.

The work undertaken in the development of the sound-sculptures is of an experimental nature. Therefore, ease of production and reproduction of the enclosures and the supporting parts is an important consideration. The use of rapid prototyping techniques not only facilitates this, but also provides a well-documented digital framework that can be referred to or built upon when desired. 
This chapter introduces the sound-sculptures in the chronological order in which they are constructed, and provides detailed information regarding the specific design features, functional mechanism, and sonic characteristics of each. Guided by the overall conceptual and functional strategies discussed above, as demonstrated in this chapter, the ideas informing the development of the series are dynamically shaped and unfolded throughout, and in parallel to the development process itself.

\subsection{Rasper}

Rasper (Figure 3-2) is the first instrument in the series of mechatronic sound-sculptures developed over the course of this research.

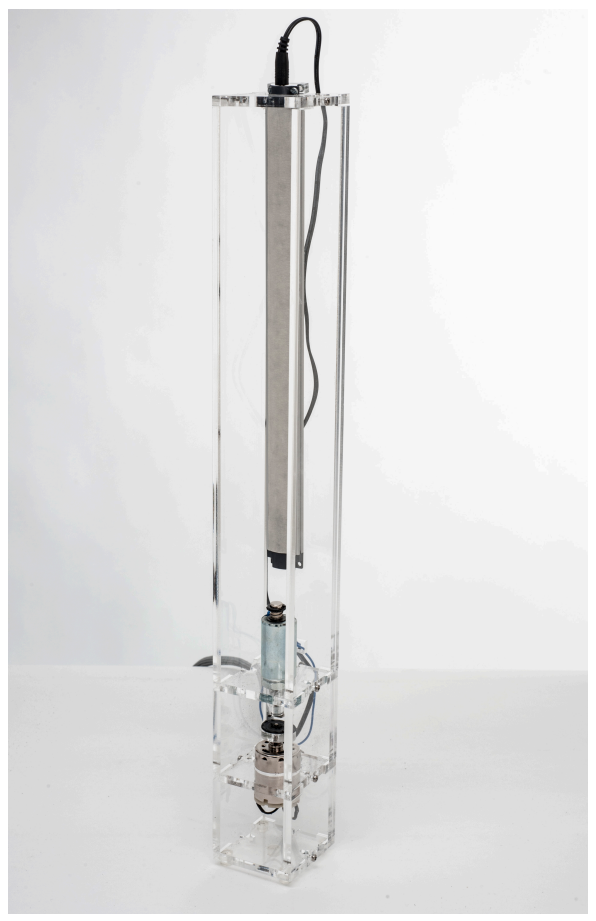

Figure 3-2. Rasper.

As mentioned above, the instrument was designed with the objective of physically creating a sonic output that is noisy (both socioculturally and acoustically), while having 
the ability to control both rhythmic and timbral elements within an audiovisual context. Rasper uses a mechatronic apparatus to achieve this objective.

\subsubsection{Design}

Rasper comprises a small $12 \mathrm{~V}$ DC motor whose shaft is connected to a 3D-printed plastic disc, a $12 \mathrm{~V}$ push solenoid attached to a sharp-edged piece of spring steel, and a bright white LED strip encased in an open-faced transparent enclosure, which was designed using computer-aided design (CAD) technology. After creating 3D models of all the components in SolidWorks ${ }^{11}$, the enclosure was laser-cut from $6 \mathrm{~mm}$ thick clear acrylic (see Figure 3-3).
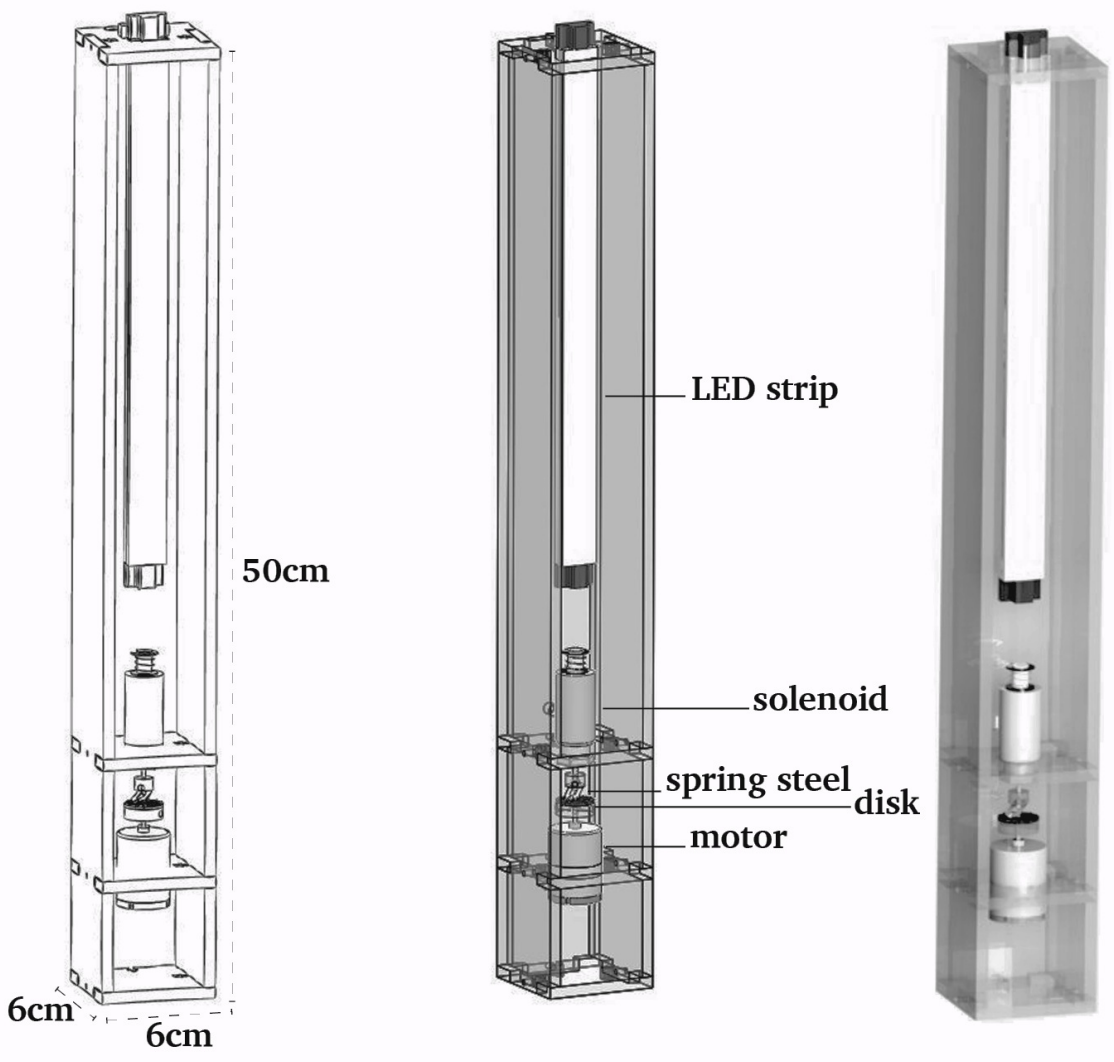

Figure 3-3. Rasper: design sketch, parts, and 3D model.

${ }^{11}$ www.solidworks.com 
The spring steel is retrieved from the adjusting knob of an off-the-shelf retractable blade knife and screwed onto a small laser-cut acrylic cylinder. The cylinder is then attached to the solenoid's shaft using a setscrew on the side. The disk is 3D-printed from black PLA and is glued onto a thicker laser-cut acrylic disk that is attached to the motor's shaft using setscrews (see Figure 3-4). The LED strip is an array of 42 cool white LEDs. A strip of white masking tape is used to cover the LED strip in order to diffuse the individual LEDs and have the entire array light up as a homogeneous tube.

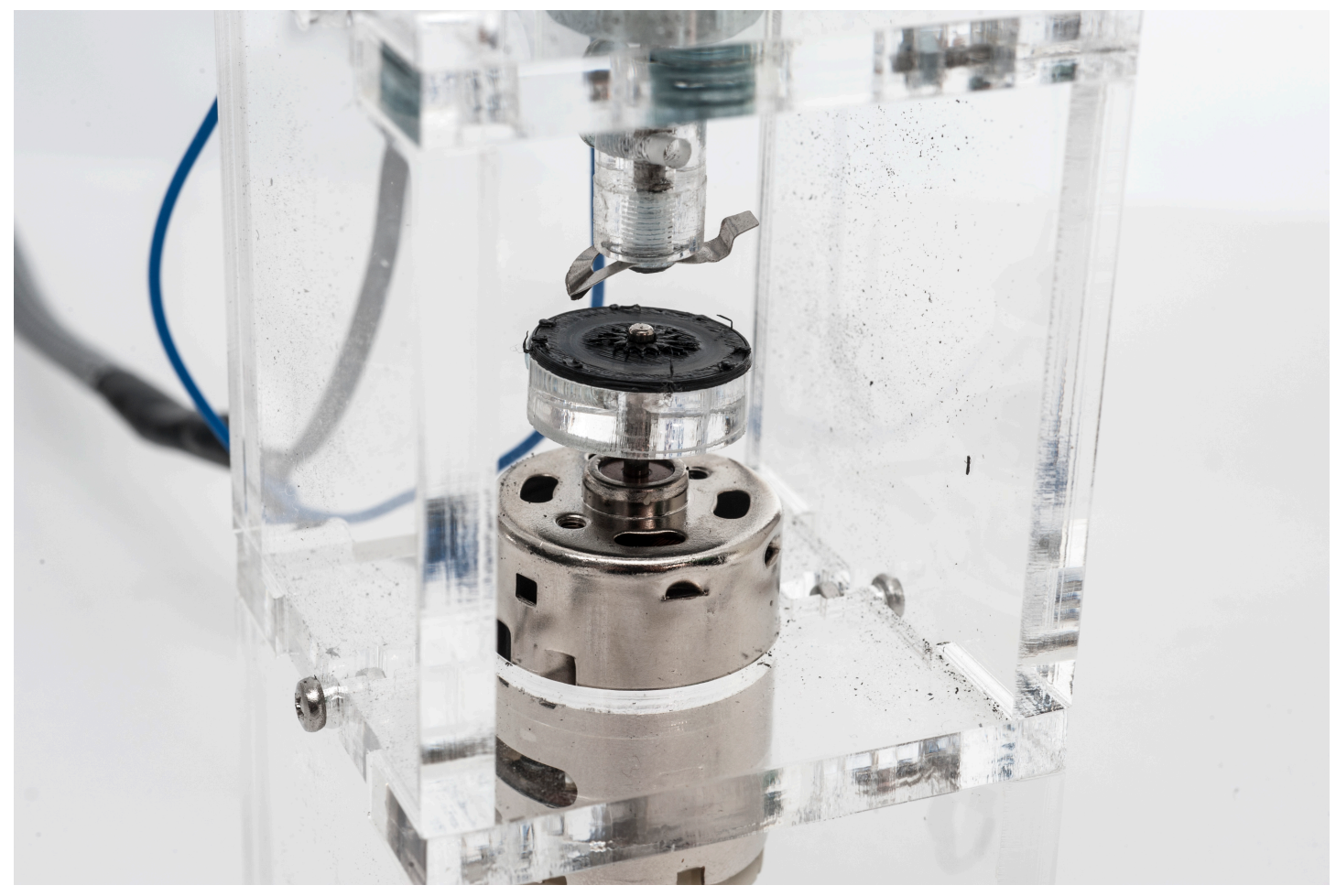

Figure 3-4. Rasper: sound-generating unit.

\subsubsection{System Overview}

Figure 3-8 outlines the system overview and the process of sound-production in Rasper. First, the input data - either from software or a MIDI controller - is transferred onto a microcontroller. The microcontroller is an Arduino Mega that has been converted into a MIDI device using HIDUINO firmware [51], and the communication is accomplished using MIDI messages. 


\subsubsection{The Driver Board}

A custom-design driver board is used to drive the instrument (Figure 3-5). The board is designed as an Arduino shield and can be plugged into an Arduino Mega in order to receive the MIDI messages that are sent from the computer. Once the input messages are received, the board converts them into a voltage that is used to drive the motor, the solenoid, or the LED strip.

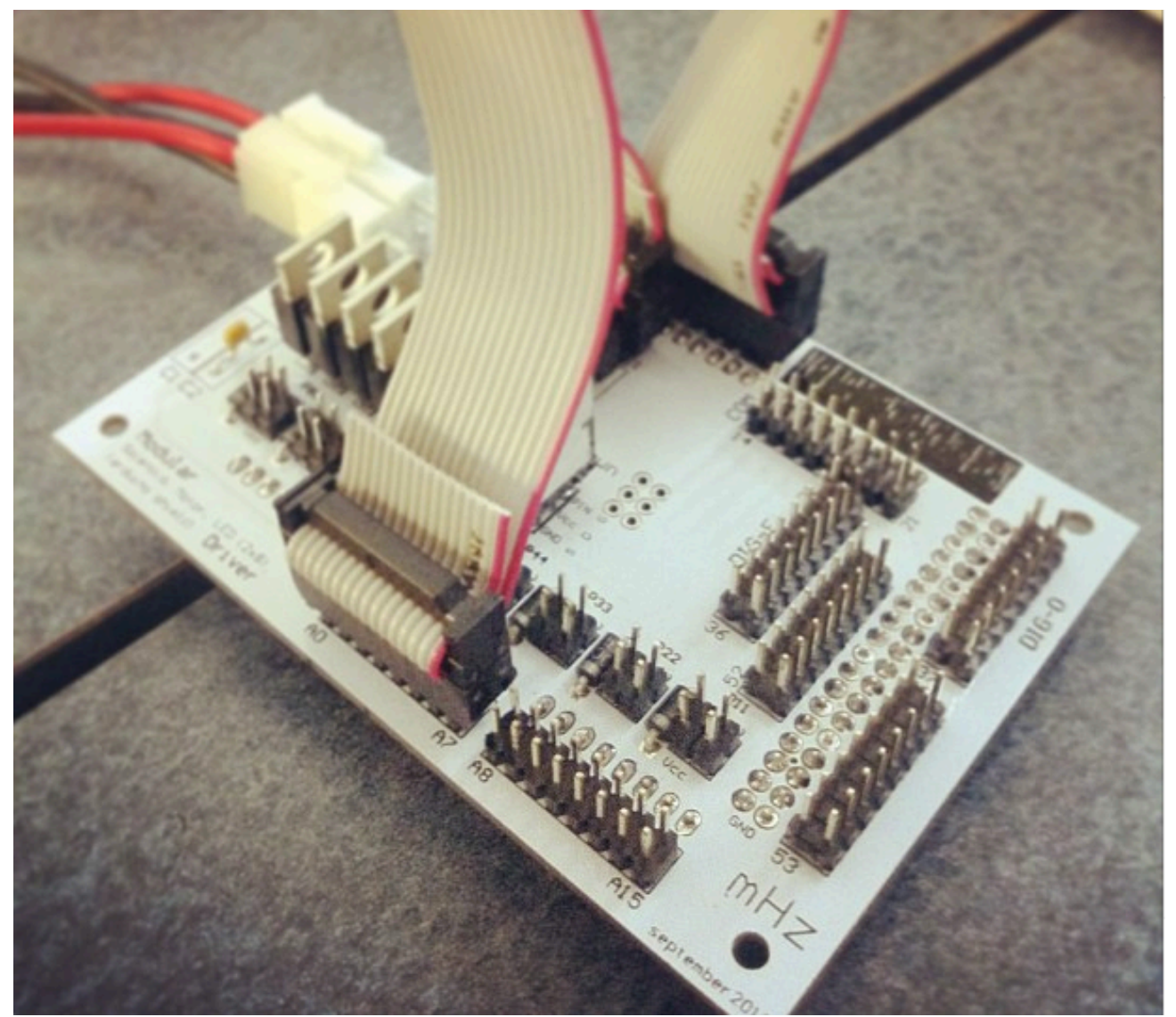

Figure 3-5. Driver board.

The driver board uses eight FDB7030BL MOSFETs, each of which takes a PWM signal from an Arduino output. The capability exists to use the same signal to drive two different components simultaneously. Therefore, the board can drive up to 16 different solenoids, motors, or LED strips. Figure 3-6 shows the circuit diagram for one of these eight modules. 


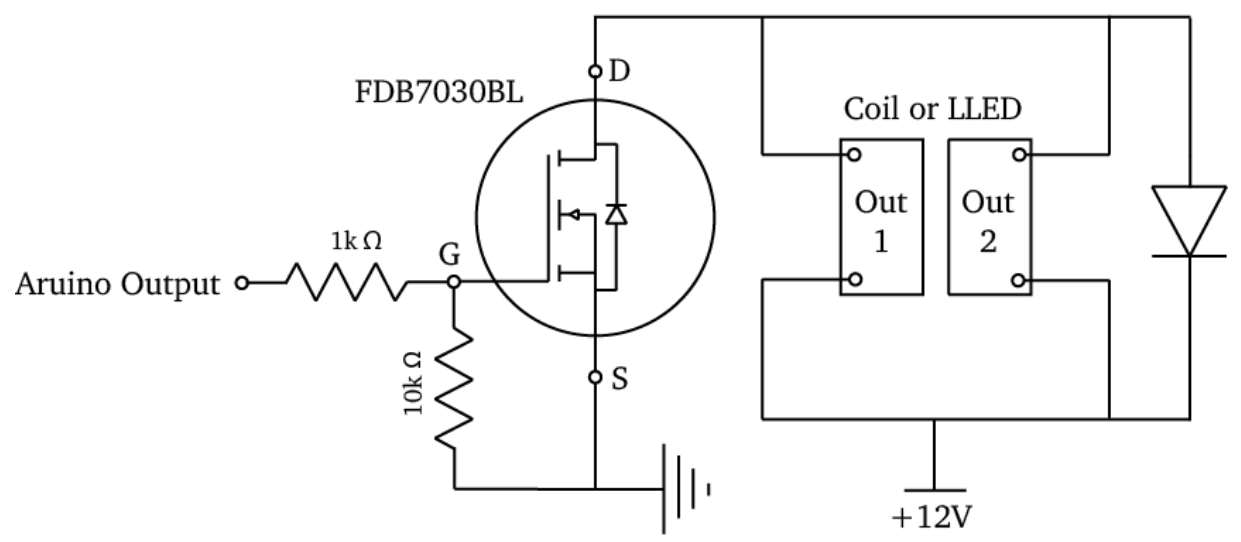

Figure 3-6. Driver board: circuit diagram.

The board is designed using Altium Designer ${ }^{12}$ and printed at Seeed Studio ${ }^{13}$. The PCB layout is demonstrated in Figure 3-7.

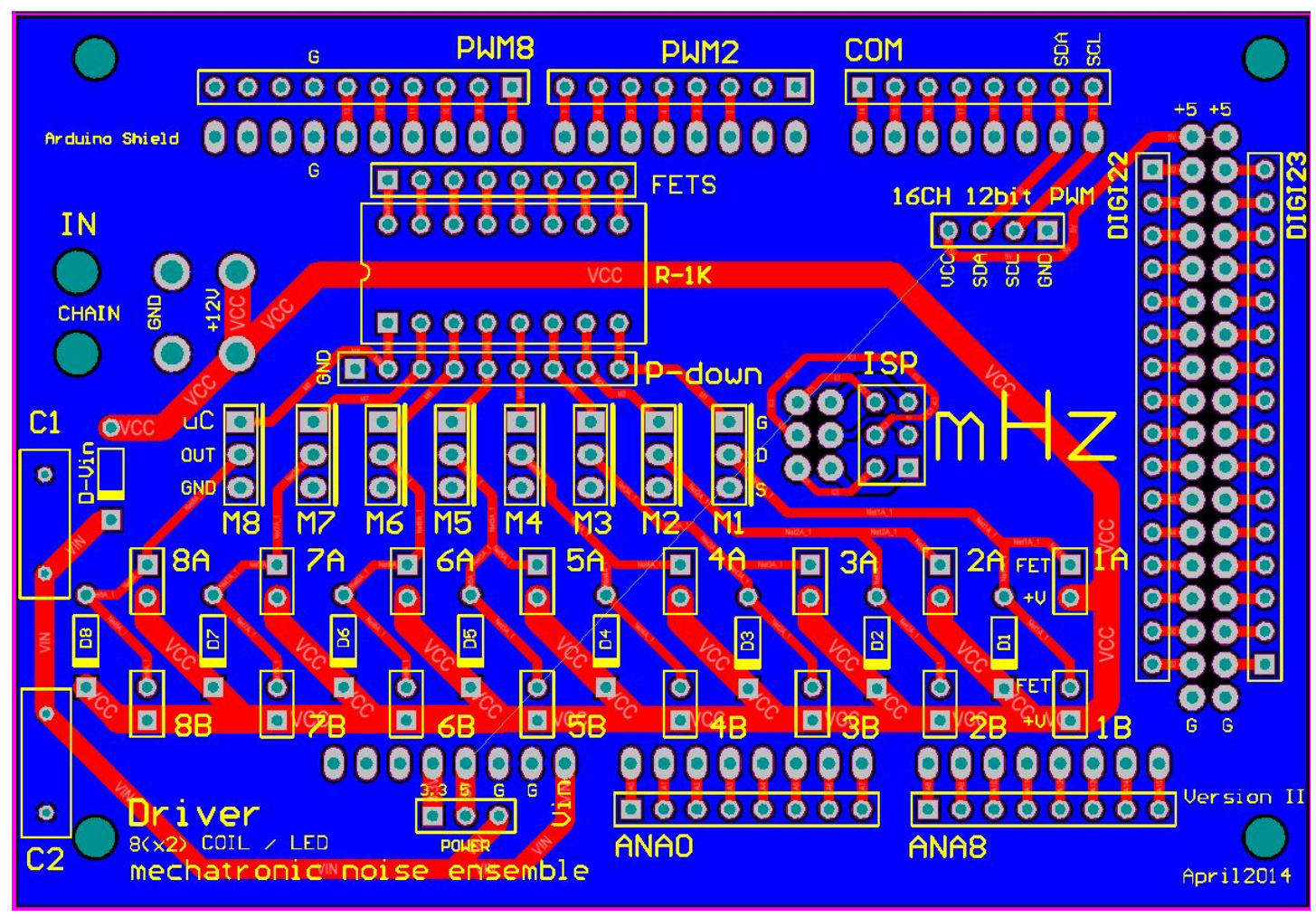

Figure 3-7. Driver board: PCB layout.

12 http:/ / www.altium.com

13 http://www.seeedstudio.com 


\subsubsection{Signal Chain}

Once the driver-board receives the MIDI input messages, it converts the MIDI velocity values into a voltage and sends them to the assigned component (that is motor, solenoid, or LEDs), which is determined by the MIDI note numbers. For motor inputs, different MIDI velocity values correspond to different speeds of rotation, creating a timbral range and a sense of variety in the frequency-domain. For solenoid inputs, a MIDI velocity value of 127 flips the current in the solenoid and pushes the shaft outward, making the contact between the spring steel and disk. Once the solenoid receives the note-off message, the current is switched back and the return spring pulls the shaft back to the rest position. Therefore, while the frequency and timbral characteristic of the sonic output depends on the motor's rotation, the solenoid's movements determine its rhythmic behaviour (see Figure 3-8).

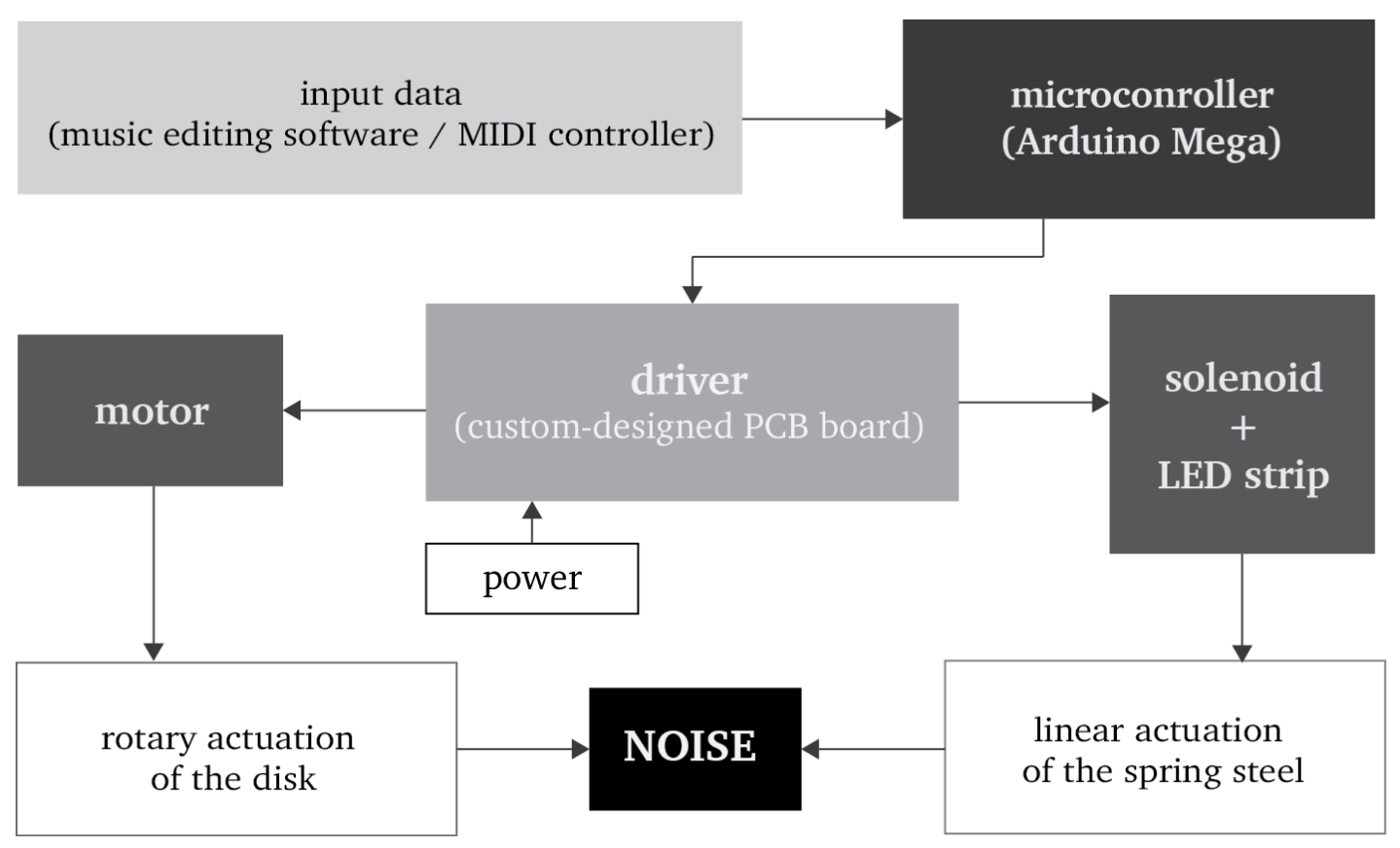

Figure 3-8. Rasper: system overview.

\subsubsection{Sound-generating Mechanism}

The sound-generating unit of Rasper is displayed in Figure 3-9. When the instrument receives the input signal, the motor spins the disk and the solenoid pushes out. As the tip of the spring steel makes contact with the rotating disk, the resulting vibration and 
surface friction produces a high frequency 'rasping' sound. In fact, the sound-generating mechanism of Rasper is, to some degree, inspired by the one used in a number of Russolo's noise-intoners. In several of Russolo's noise-machines, a piece of metal string whose tension could be controlled using a lever, was placed in contact with, and was vibrated by a wooden wheel. Russolo's wooden wheel was in many cases rotated manually using a crank. By handling the lever and cranking the crank, the performer would create noise while controlling its timbre and tone. In Rasper, the vibrating material, that is the string, is replaced with the piece of spring steel, while the wooden wheel, the crank, and the lever become the plastic disk, the DC motor, and the push solenoid respectively. The manual interaction is replaced by microcontroller programming (see Figure 3-9). The use of solenoids allows the possibility of switching the contact between the sound-generating components on and off very quickly, and therefore, enables the system to produce rhythmic patterns.

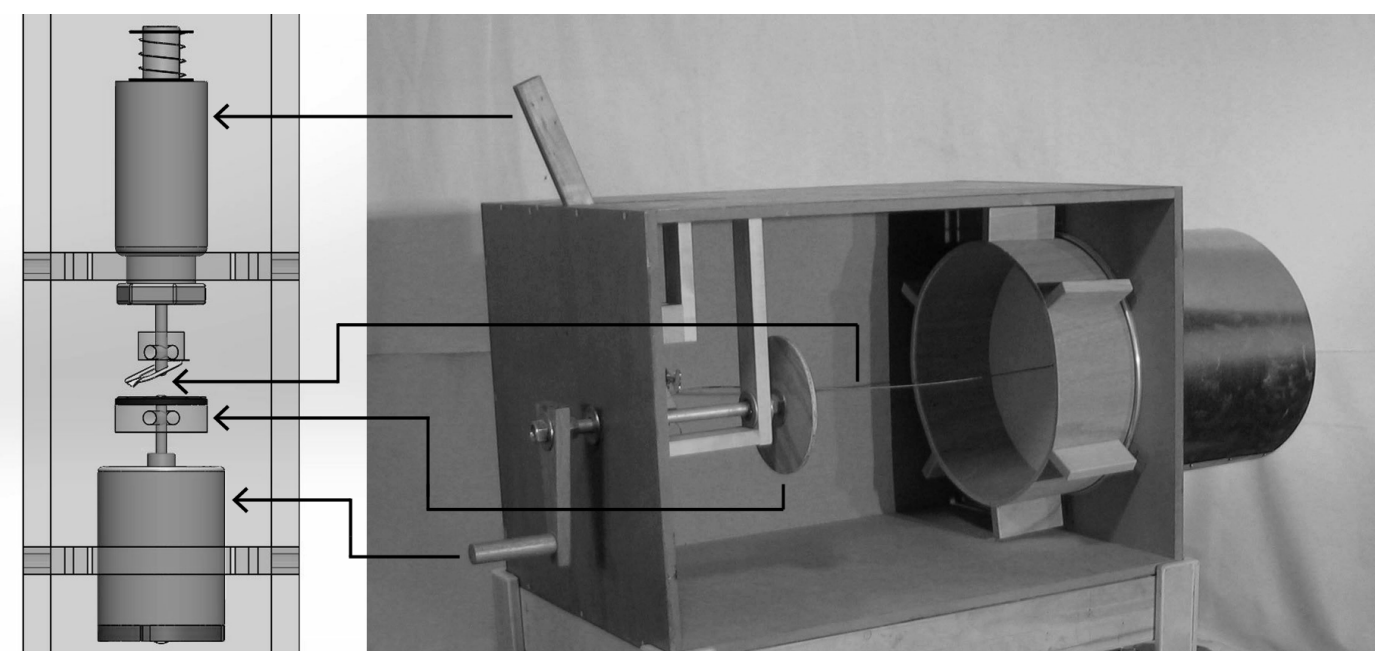

Figure 3-9. Sound-generating mechanism of Rasper (left) contrasted to the one used in a model of Russolo's Crackler, developed by New Music Co-op [52].

\subsubsection{The Light Element}

The physical and bodily method of sound-production is one of the key elements in the design of Rasper, and the visual aspect of the work as a piece of sculpture is of great prominence. While in Russolo's instruments the sound-generating unit is enclosed and hidden inside a box, the entire apparatus in Rasper is fully visible and placed in a fully 
transparent enclosure. Therefore, aside from the mechanism of sound-production, one of the main aesthetic and ideological differences between Rasper and Russolo's instruments lies in the visibility of sound-production.

With this in mind, in order to further highlight the visual aspect of the work, the mechanical method of sound-production is accompanied by synchronous patterns of the LED strip, boosting the audiovisual connectivity. In order to achieve this, the same signal that is used to drive to solenoid is sent to the LEDs. Therefore, whenever there is a pulse (that is the contact between the spring steel and the disk is made), the LED strip lights up, and once the contact is off, the LED strip goes off as well. The choice of white light was primarily an aesthetic decision. However, metaphorically speaking, in the same way that no one dominant frequency stands out in a noisy sound, white light encompasses the widest visible frequency range and gives no advantage to any light of a particular colour. On the other hand, if machine noise is perceived as dull, tasteless, and aesthetically unpleasant in an everyday context, so is the fluorescent light. Therefore, Rasper tries to aestheticise not only the aurally uninteresting, but also the visually insipid phenomena existing in modern urban life by structuring them onto a rhythmic grid and bringing them back to the attention domain.

\subsubsection{Evaluation}

Rasper is an instrument whose sonic output can be modified and controlled in terms of timbre and rhythm, using appropriate input values. Therefore, two different sets of tests are conducted (one on frequency-domain features and one on time domain behaviour) in order to evaluate the instrument's response from both angles.

\subsubsection{Frequency-domain}

In order to study the frequency characteristics of Rasper, a series of tests are conducted on 1-second long recordings of the instrument at various speeds ${ }^{14}$. As mentioned earlier in this section, the essence of the sound-production in Rasper is to create noise, that is, to avoid definite pitch and maintain the richness and noisiness of the sonic output. The

\footnotetext{
14 As Rasper's sound is quite noisy, 1-second long recordings are made to provide an average analysis of the instrument sonic quality.
} 
average FFT results of the recordings of the instrument at various speeds, shown in Figure 3-10, verify this noisy characteristic. This is an indicative test, extracted from average FFT analyses of recordings of three different speeds acquired by applying different MIDI velocity values $(77,101$, and 127) to the motor. By comparing the fast and medium distributions to the slow graph, a diminution of higher frequencies for lower speeds, where the vibration of the spring steel and the surface friction are both of lower intensity and amplitude, can be noticed. The higher-frequency rotations lead to higher amplitude signals. A local maximum can be noticed around $9 \mathrm{kHz}$ in all three plots, which can be interpreted as the system's natural frequency of excitation. Nevertheless, all three plots show the lack of presence of definite pitches and significant harmonic behaviour. Therefore, regardless of the relatively different harmonic distribution at different speeds - which can be perceived as different timbres - the sonic output of Rasper is acoustically noisy.

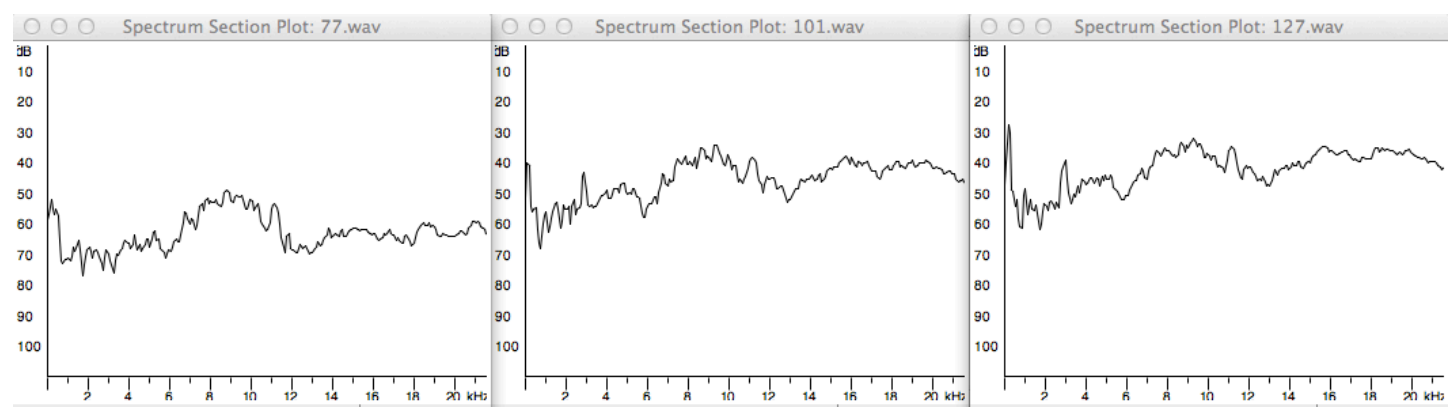

Figure 3-10. Average FFT results of recordings of Rasper at different speeds.

In a more detailed series of tests, a number of key feature extractions of the recordings of the instrument at a range of different speeds are compiled. MIDI velocities less that 71 did not produce sufficient momentum to drive the motor when the contact between the disk and the spring steel was made. Therefore, by sweeping the MIDI velocities every two steps from 71 all the way up to the highest MIDI velocity value (127), a total of 29 recordings were collected. Considering the relative unpredictability and noisiness of the instrument, these feature extraction analyses were carried out on an average of the FFT data of 1-second long recordings, with the following specifications: 
- Sample Rate: 44100 sample/s (to provide a Nyquist frequency higher than the highest audible frequency, that is $20 \mathrm{kHz}$.),

- Window Function: Hanning (default windowing mode in software specifications),

- FFT size: 1024 samples (Each 1-second long recording is approximately 43 of these 1024-point FFTs (44100/1024 43). Therefore, the feature extraction results for each MIDI velocity value are calculated as the average of 43 values).

\section{Spectral Centroid and Spectral Roll-Off}

The spectral roll-off and spectral centroid plots demonstrated in Figure 3-11 reveal that the concentration of the centre of the mass (centroid), and the frequency marking 50 percent of the energy distribution (roll-off) of the audio signal is, for the most part, in the high frequency-domain ${ }^{15}$. This can be perceived as the significant brightness of Rasper's sound. As the speed of rotation goes higher, the amplitude and frequency of the vibration increase, introducing higher frequencies into the spectrum and increasing the brightness.

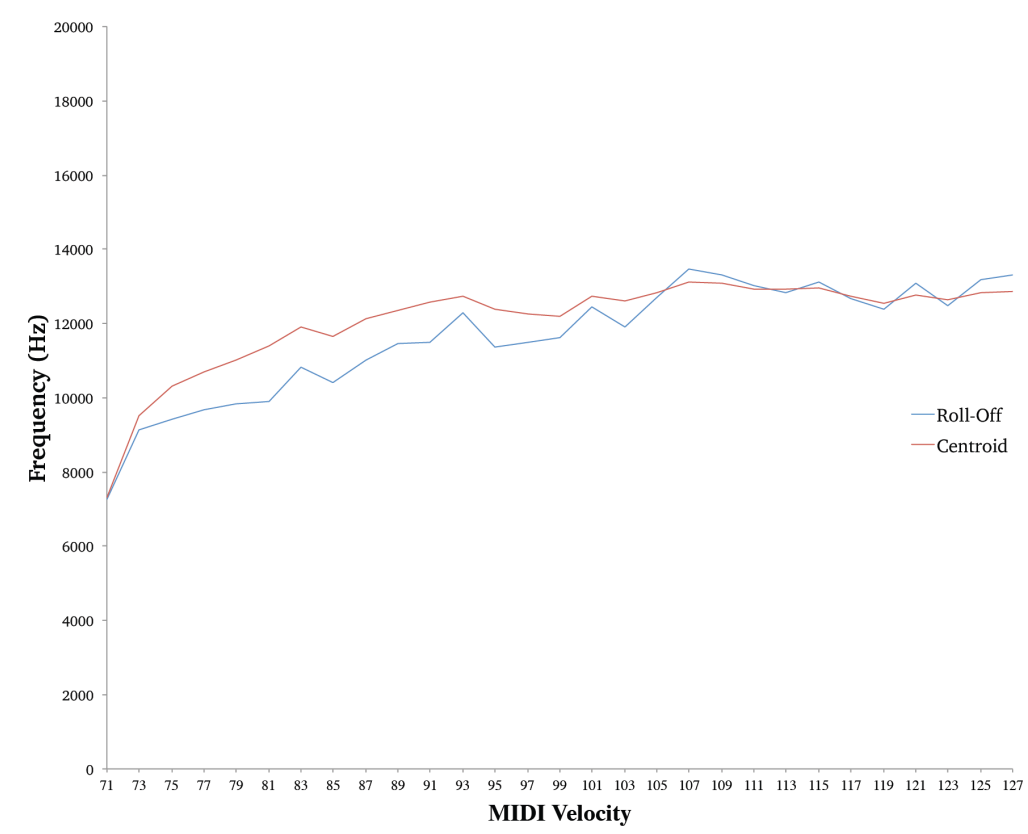

Figure 3-11. Rasper: spectral roll-off and spectral centroid.

15 Considering the perceptual, subjectivity, and varying nature of different frequency-band definitions from source to source, the following general classification is suggested: lows (frequencies below $500 \mathrm{~Hz}$ ), mids (from $500 \mathrm{~Hz}$ to $6 \mathrm{kHz}$ ), and highs (above $6 \mathrm{kHz}$ ). 
As can also be observed in Figure 3-10, the difference between the energy distribution of the signal at medium and fast speeds seems to only fluctuate within a relatively narrow range.

\section{Zero-Crossings}

Figure 3-12 displays the (scaled) number of zero-crossings of the audio signal for the same input values. Considering that the audio signal is acoustically noisy and lacks periodicity, the zero-crossing rate here does not provide a translation of the signal's pitch. Instead, this plot shows that as speed passes a certain threshold, higher frequencies with higher amplitude are introduced to the spectrum and the signal becomes richer and noisier (as observed in the two previous figures). The relatively narrow distribution of this number for higher speeds can be interpreted as a subtle "sense of pitch", which navigates within an interval between $11 \mathrm{kHz}$ and $12 \mathrm{kHz}$.

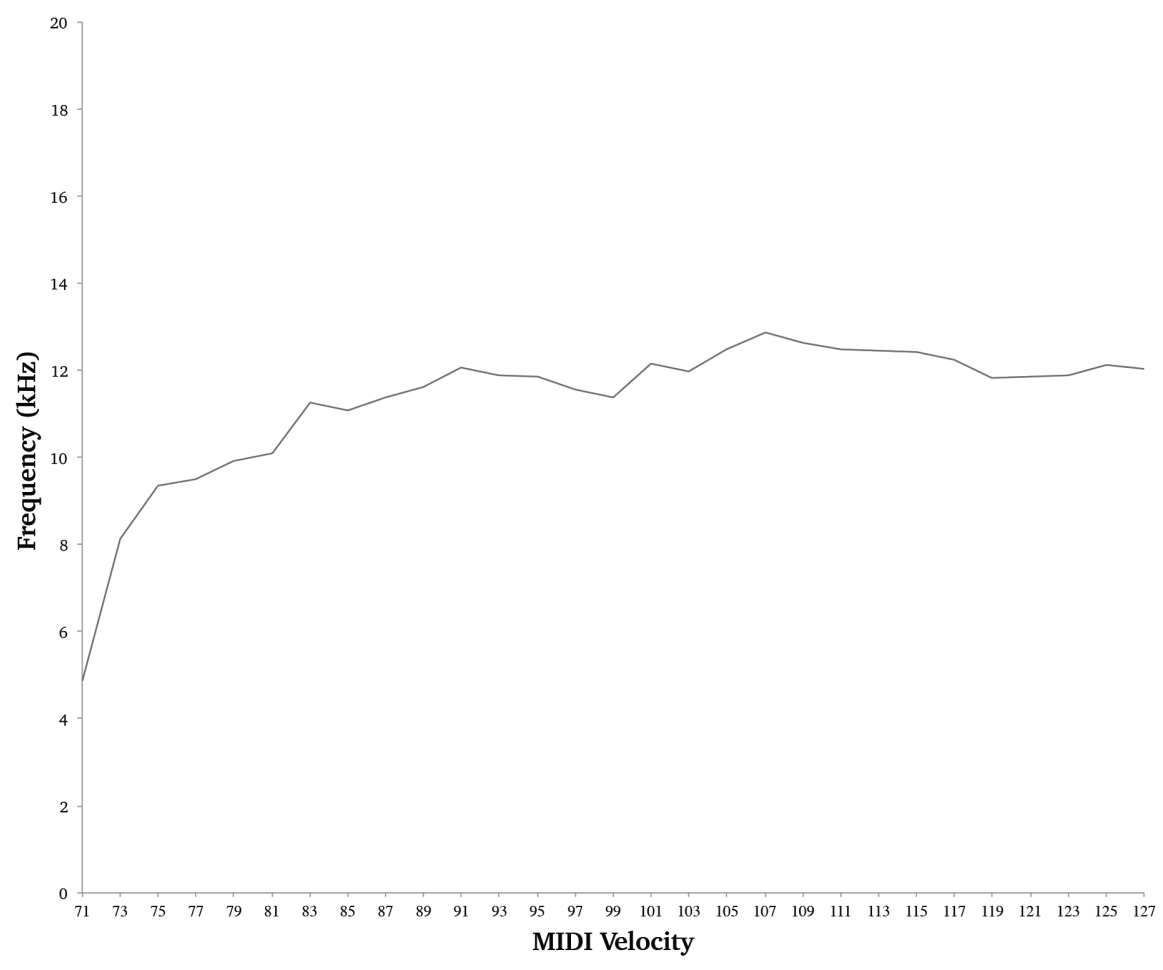

Figure 3-12. Rasper: zero-crossing. 


\section{Spectral Flux}

Figure 3-13 shows the spectral flux of the audio signal, that is, the rate of change of the output's spectrum as the input changes. The different behaviour at the lower speeds can be clearly noticed here. While the flux of the signal appears to be chaotic and noisy for the lower MIDI velocities, there can be noticed a threshold after which this wavering behaviour is considerably smoothed out. As the speed of the motor passes a certain threshold on the input and the signal becomes noisier, the numeric derivative of the change of the audio signal becomes relatively consistent on the output, that is, the change in the output is relatively linear and predictable.

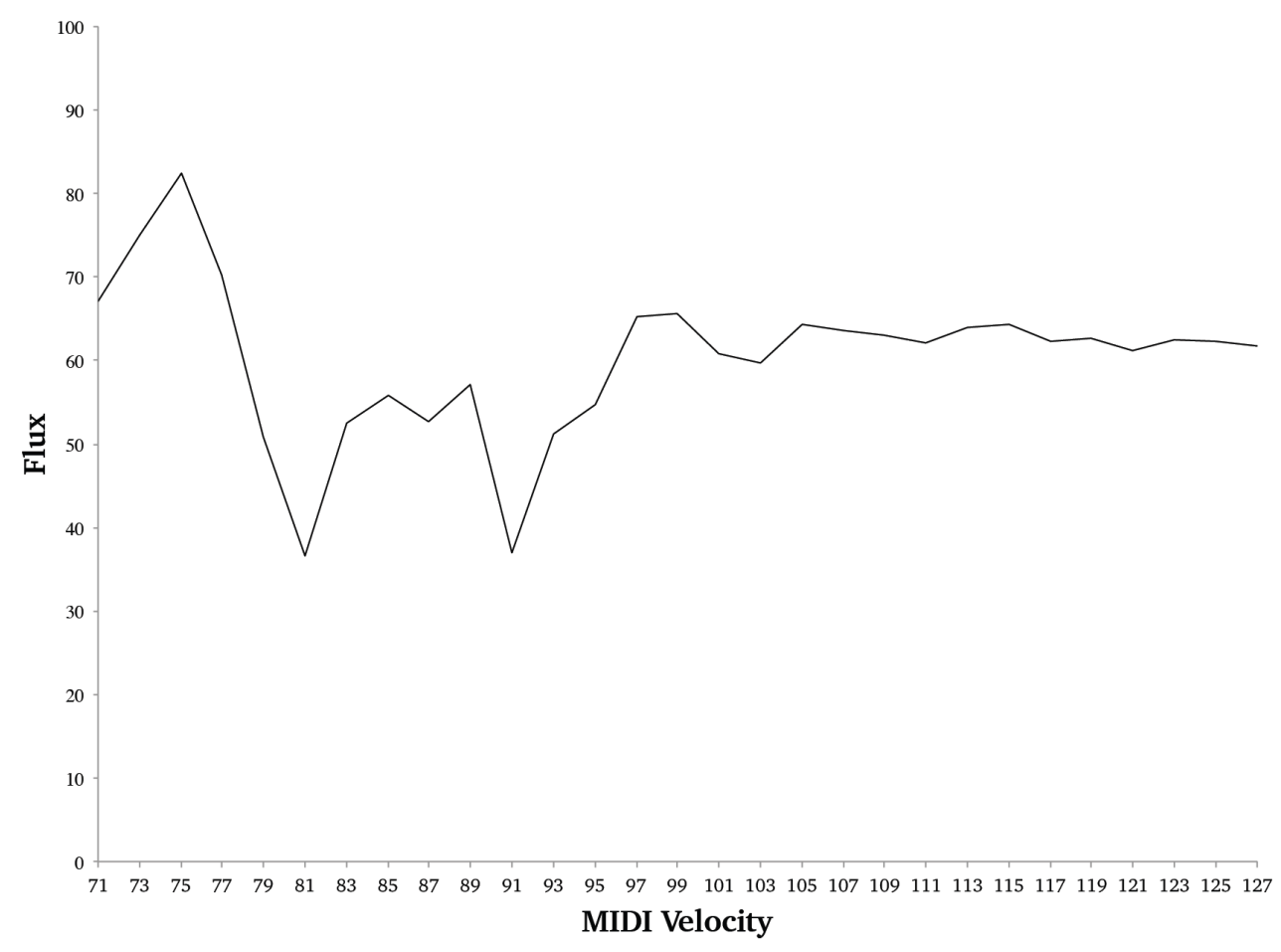

Figure 3-13. Rasper: spectral flux.

\subsubsection{Time Domain}

The temporal development of a single noise strike produced by Rasper is exhibited in Figure 3-14. The strike is generated by applying half-note long MIDI messages with velocities of 127 both to the motor and the solenoid at 120BPM (that is a 2-second long contact between the spring steel and the disk). The output is represented in waveform 
and spectrum formats. Evident from this figure is a small latency between outputting the MIDI note-on and note-off messages from software and its mechanical implementation by the instrument. There can also be observed a very short attack time, as well as a relatively longer release time. These time intervals are marked with the following labels: $\mathbf{L}_{1}, \mathbf{L}_{2}, \mathbf{A}$, and $\mathbf{R}$. These specific time intervals of course correspond to this one specific input value. However, by comparing the waveforms of the recordings of different inputs, the time differences appear to be marginal. Therefore, Figure 3-14 provides a good example of the general behaviour of Rasper's output in the time domain. The time intervals marked in the figure are:

- $\mathbf{L}_{1}$ : note-on latency

- $\quad \mathbf{L}_{2}$ : note-off latency

- A: attack time

- $\mathbf{R}$ : release time.

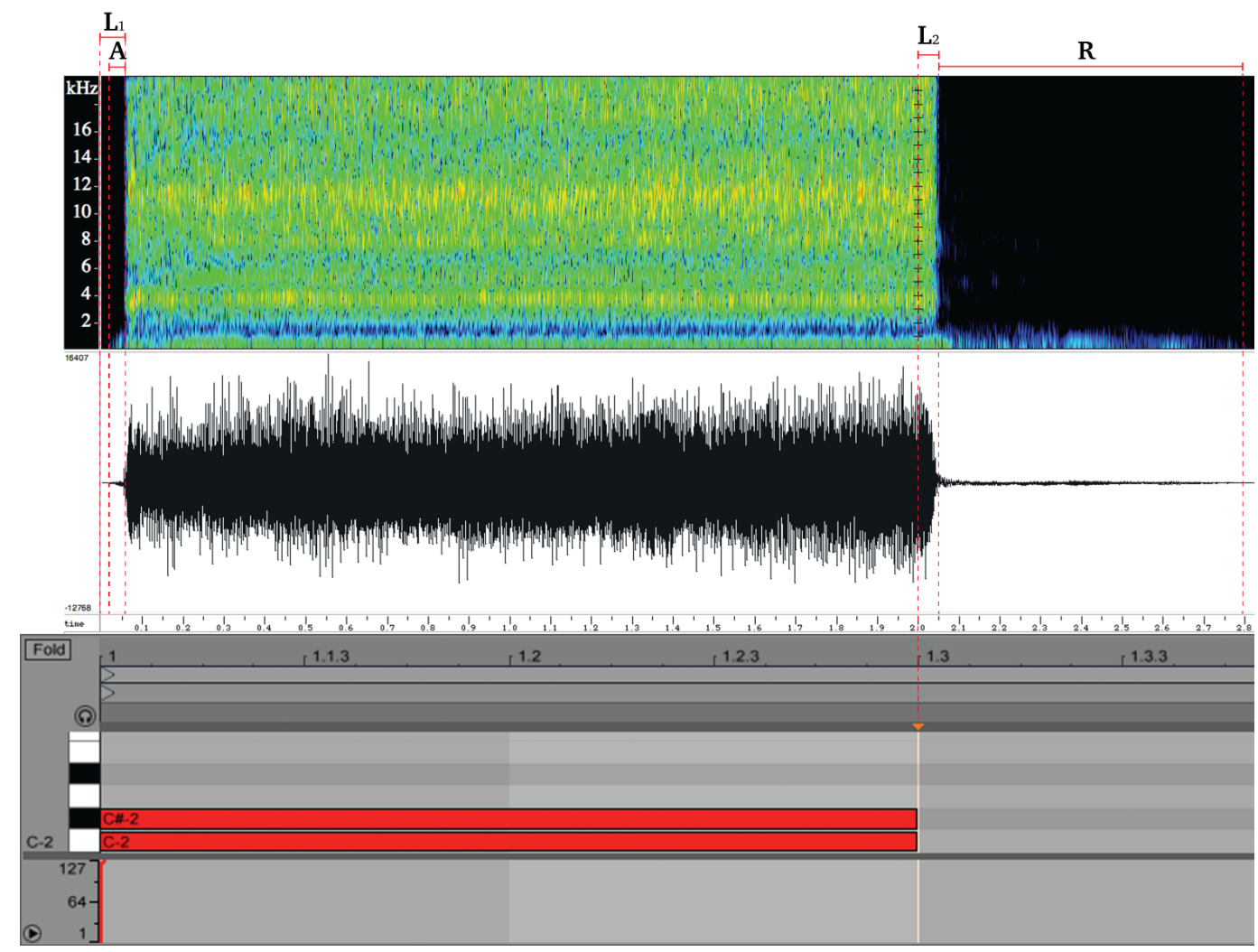

Figure 3-14. Rasper's output generated from a 2-second long input with maximum MIDI velocity values for the motor and the solenoid: waveform and spectrum representations. 
These intervals can be explained as follows: $\mathbf{L}_{1}$ comprises the audio interface input latency (approximately 20ms for a buffer-size of 1024 samples), and the time required for the solenoid shaft to move down the spring steel and put it in contact with the disk. The latter, which is the mechanical latency, is referred to as the attack time and labelled A (see Figure 3-15). The weak audio signal in this time interval represents the sound of the motor starting to run. $\mathbf{L}_{2}$ shows the time interval between the midi note-off command from the software, and its physical realisation, that is removing the contact between the spring steel and the solenoid. The decaying form of the signal towards the end of $\mathbf{L}_{2}$ is due to the motor slowing down from the maximum speed after having its torque cut off by the note-off message. The interval between the releasing of the contact to the point where the motor fully stops is marked by $\mathbf{R}$ and is referred to as the release time. The weak and decaying signal of the sound of the motor slowing down can be observed during $\mathbf{R}$. The release time would be shorter for inputs with lower MIDI velocities (slower speeds of rotation), where less time is required for the motor to fully stop.

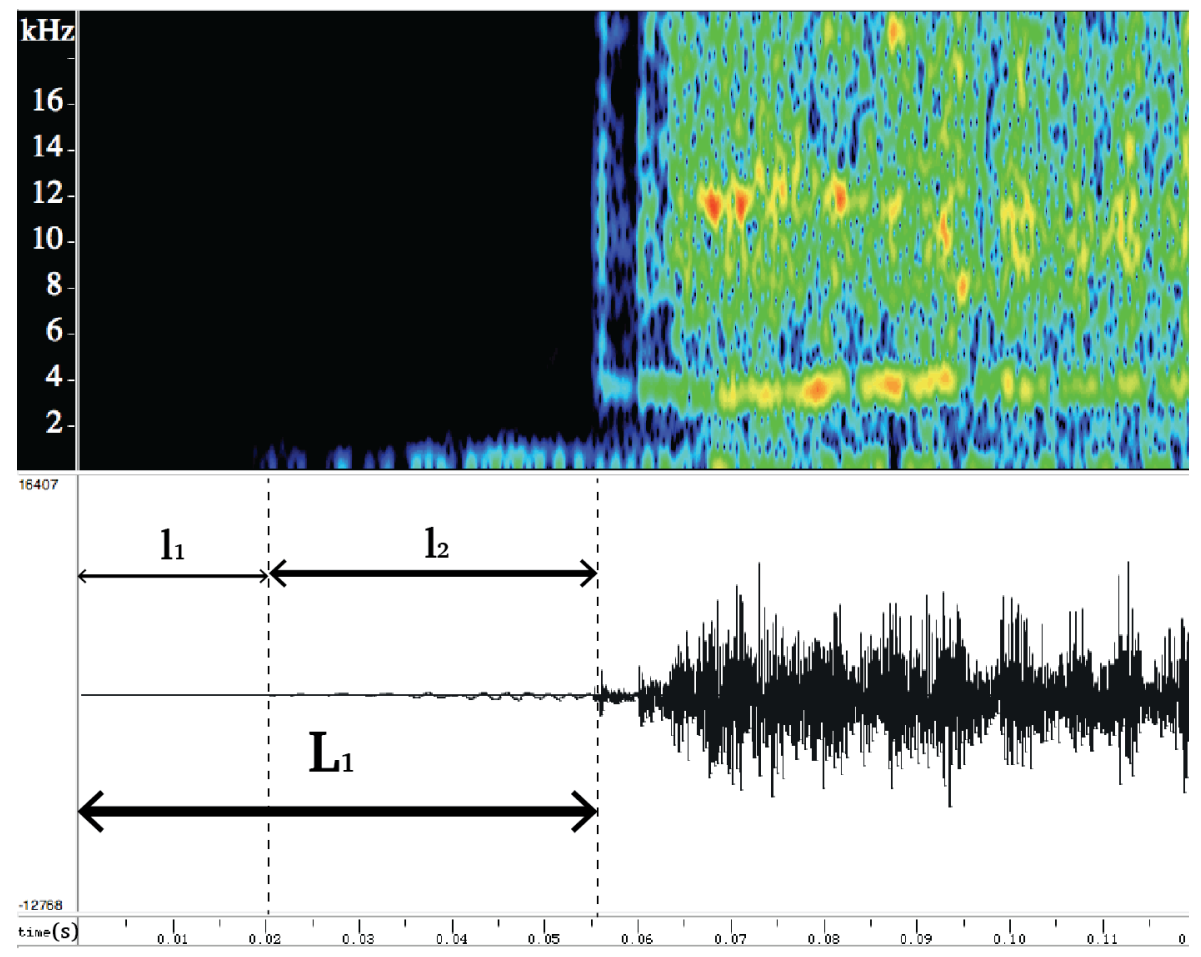

Figure 3-15. The full latency $\left(L_{1}\right)$ is comprised of the software latency $\left(l_{1}\right)$ and the mechanical latency $\left(\mathbf{l}_{2}\right)$. 
Considering the rhythmic attribute of Rasper, these latencies should be accounted for in order to achieve better synchrony (specially when accompanied by other rhythmic sources of sound).

\subsubsection{Summary}

In terms of acoustics, Rasper generates noise rather than musical tones ${ }^{16}$. The output noise primarily lies in the high frequency-domain. The output noise is produced in a rhythmical manner, and can be modified timbrally. However, the timbral variation is rather limited, as the instrument sound is considerably noisy. Solenoid inputs determine Rasper's rhythmic behaviour, while variations in motor inputs introduce the limited sense of variety in terms of timbre. Steadier outputs could be achieved by working with higher speeds. There is a small actuation latency that needs to be taken into account when dealing with synchrony.

\subsection{Rippler}

Although the primary sonic output of Rasper is caused by the contact between the spring steel and rotating disk, the actuation sound of the mechatronic components, that is the solenoid and the motor, is not entirely negligible ${ }^{17}$. As discussed in the previous chapter, in a context where achieving a certain musical output is key, such actuation noise can be a hindrance. However, in the context of this research, this actuation noise not only was not unwelcome, but also a source of sonic material for the design and development of the succeeding sound-sculptures. Accordingly, as the second sound-sculpture in the series of mechatronic sound-sculptures developed over the course of this research, Rippler was designed to highlight the actuation noise of a solenoid. In this soundsculpture, the actuation noise of a $12 \mathrm{~V}$ push solenoid is transformed and amplified by

\footnotetext{
16 To reiterate, Hermann Helmholtz argues that the differences between noises and musical tones are rooted in our aural perception, stating that musical tones are perceived as periodic, and noises are perceived as non-periodic motions [4].

${ }^{17}$ Specially for the lower speeds of rotation, as discussed in 3.2.3.
} 
the mechanical excitation of a thin sheet of steel. For the purpose of consistency, Rippler uses the same type of solenoid that is used in Rasper.

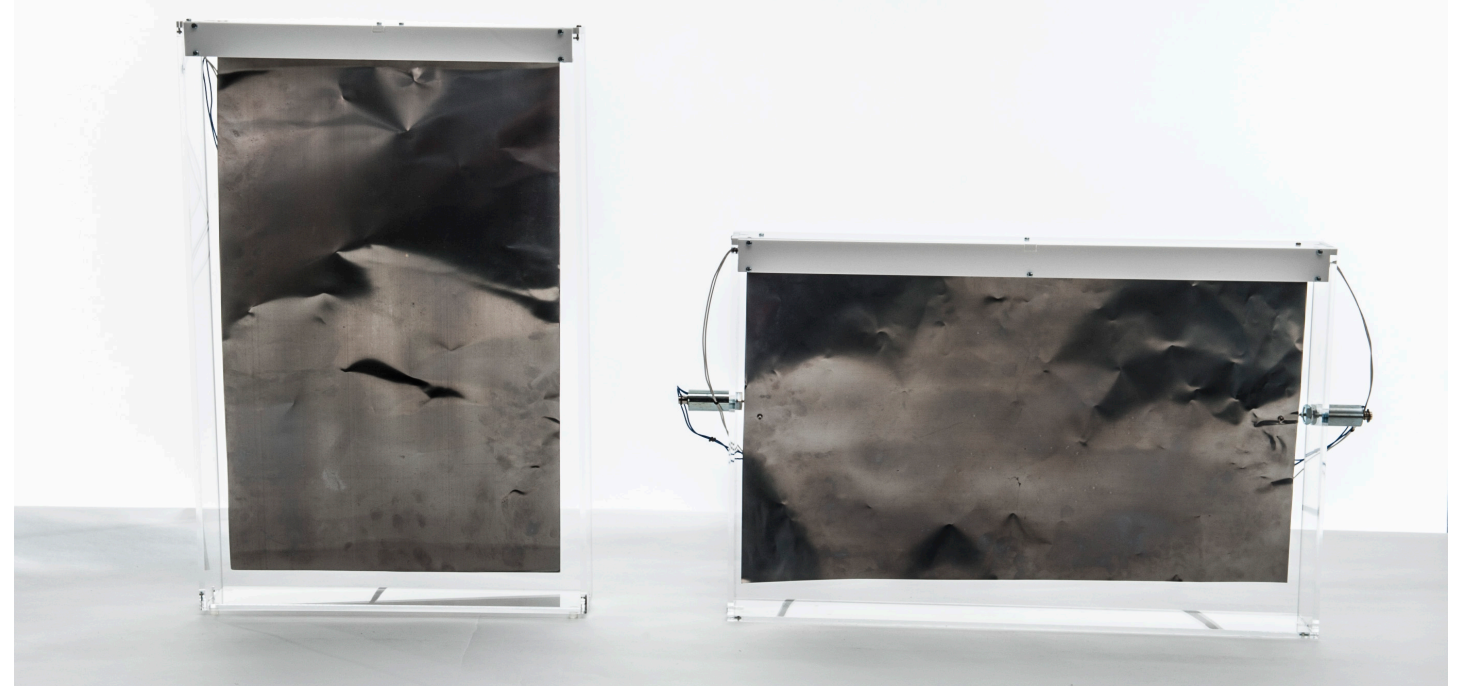

Figure 3-16. Rippler: V-model (left) and H-model (right).

\subsubsection{Design}

In order to experiment with different actuation modes and achieve more sonic variety, two versions of Rippler are designed that vary in terms of the mechanics they use in order to excite a sheet of steel. The two models are titled based on the orientation of the steel sheet in the instrument: horizontal (H-model) and vertical (V-model) (see Figure 3-16). The horizontal and vertical models both comprise a thin sheet of steel framed in a transparent acrylic frame, with push solenoids (two in $\mathrm{H}$-model and one in V-model) attached to it. In both models, the topside of the frame is replaced with a square tube made of white acrylic that encloses a strip of cool white LEDs. The white LEDs were chosen in accordance with the aesthetic principles discussed in 3.2.2.4. Similar to Rasper, the LED strips' luminosity in Rippler corresponds to the movements of an associated solenoid. In addition to the orientation of the frame and the number of solenoids, the two models also vary in terms of the thickness of the sheet and the direction of actuation. This is implemented in order to achieve higher degrees of timbral variety. 
In both models, the frames are designed using CAD technology (Figure 3-17), and 3D models of all the components are designed in SolidWorks in order to determine the optimal sizes and dimensions, both in terms of functionality and aesthetics. Using CAM technology, the final design was laser-cut from $6 \mathrm{~mm}$ transparent acrylic, with the exception of the LED tubes, which are cut out of white acrylic - in order to diffuse the light of individual LEDs.

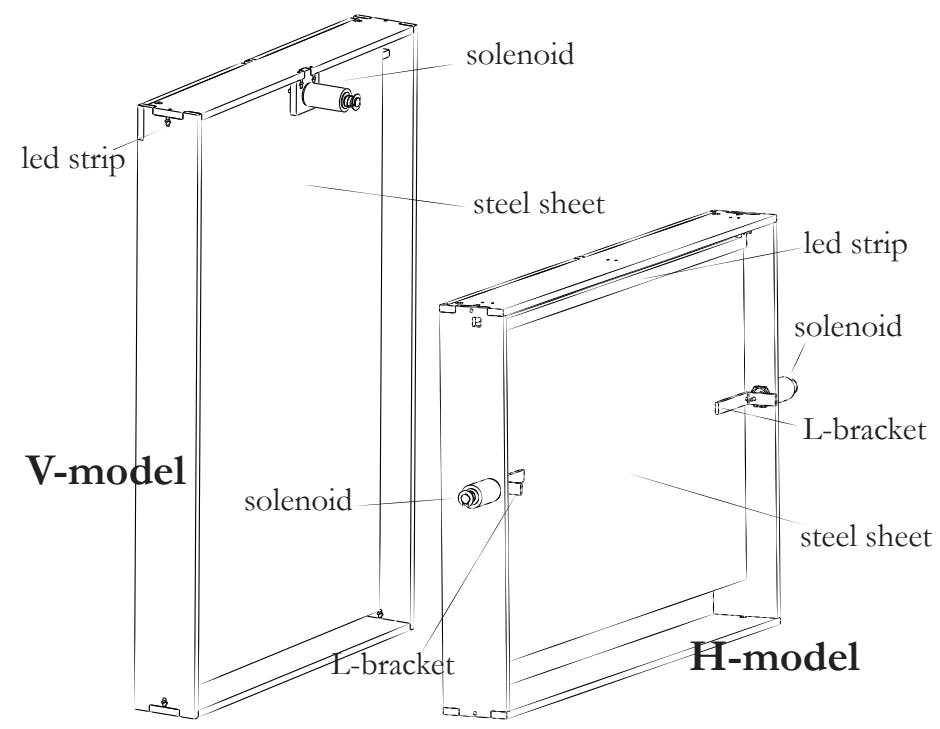

Figure 3-17. Rippler: design sketch and parts.

\subsubsection{V-model}
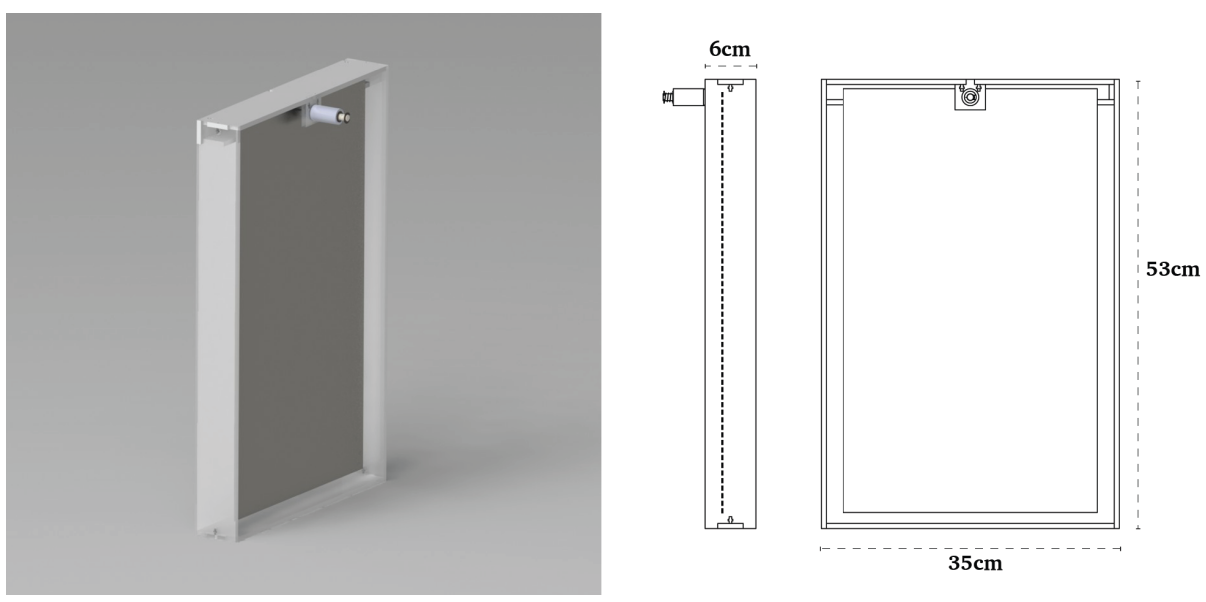

Figure 3-18. Rippler V-model: 3D model (left), design sketch: side and back views (right). 
In the V-model, a $0.08 \mathrm{~mm}$ thick $300 \mathrm{~mm} \times 500 \mathrm{~mm}$ sheet of steel is framed in a vertical acrylic frame. One solenoid is mounted on the back of the frame at the top, using a small laser-cut bracket, in order to hold the sheet hanging from the top. The solenoid's shaft is tapped and passed through a $2.5 \mathrm{~mm}$ diameter hole that has been drilled into the sheet. The sheet is connected to the solenoid using two bolts (one on each side) that are screwed into the shaft. The topside of the frame is a three-sided square tube with its open side facing backwards, making room for the LED strip bracket. A $300 \mathrm{~mm}$ long LED strip is glued on a $300 \mathrm{~mm} \times 15 \mathrm{~mm}$ piece of $6 \mathrm{~mm}$ acrylic that is screwed onto, and aligned with, the LED tube, facing the LEDs forward (Figure 3-19).

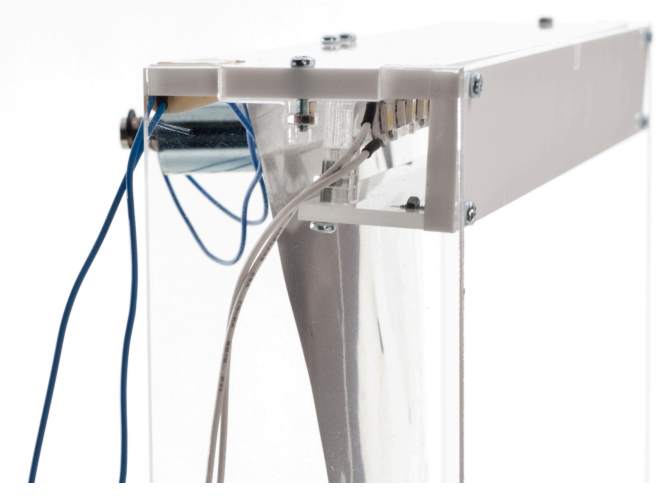

Figure 3-19. Rippler V-model: close-up.

\subsubsection{H-model}
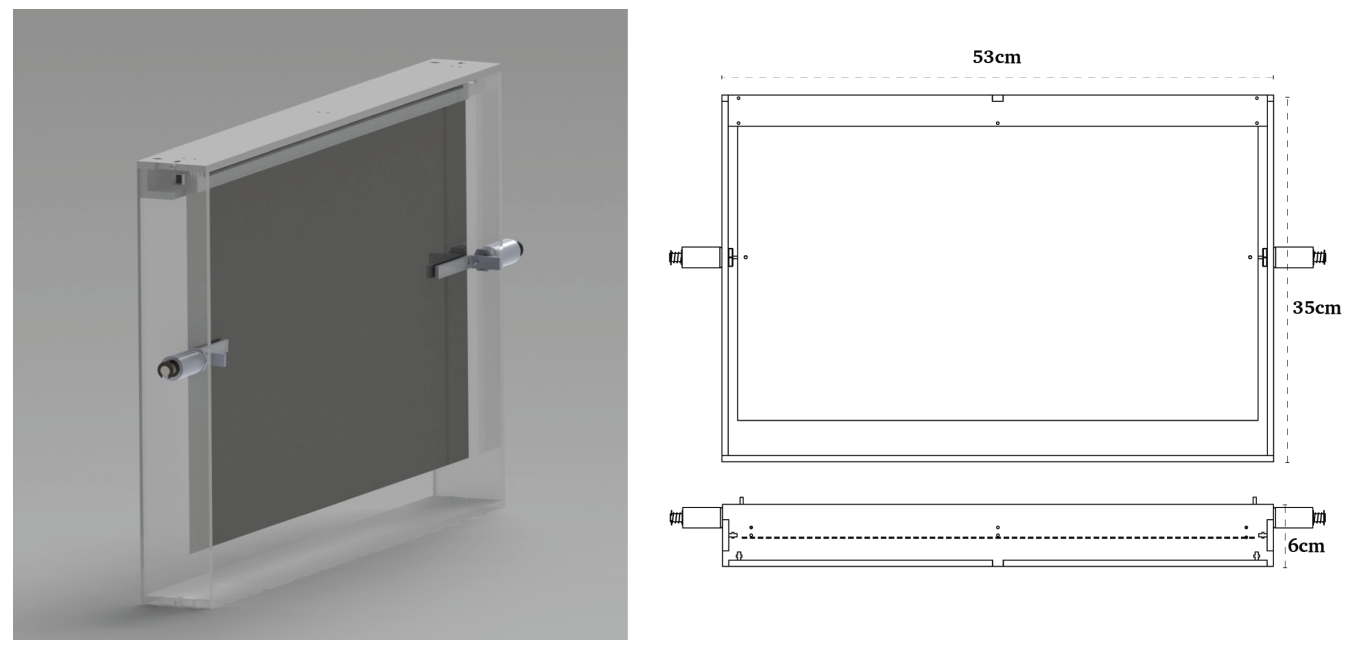

Figure 3-20. Rippler H-model: 3D model (left), design sketch back and top views (right). 
In the H-model, the steel sheet is framed in a horizontal acrylic frame and is $0.05 \mathrm{~mm}$ thick. In this model, two push solenoids are fixed to each side of the frame. Each solenoid is attached to one end of the steel sheet, using small laser-cut acrylic L-brackets with $2.5 \mathrm{~mm}$ diameter holes on both their ends. The tapped solenoid shaft is screwed to one end of the L-bracket, and the sheet - which also has $2.5 \mathrm{~mm}$ diameter holes drilled on both ends - is attached to the other end of the bracket, using a screw and two bolts. Similar to the V-model, the topside of the frame is a three-sided square tube with its open side facing backwards (Figure 3-21). Two 250mm long strips of cool white LEDs are glued on a $500 \mathrm{~mm} \times 15 \mathrm{~mm}$ piece of $6 \mathrm{~mm}$ acrylic that is fixed in the LED tube. The strips are facing forward and are glued next to each other, forming one 500mm-long strip that comprises two independently controlled halves that correspond to their associated solenoids.

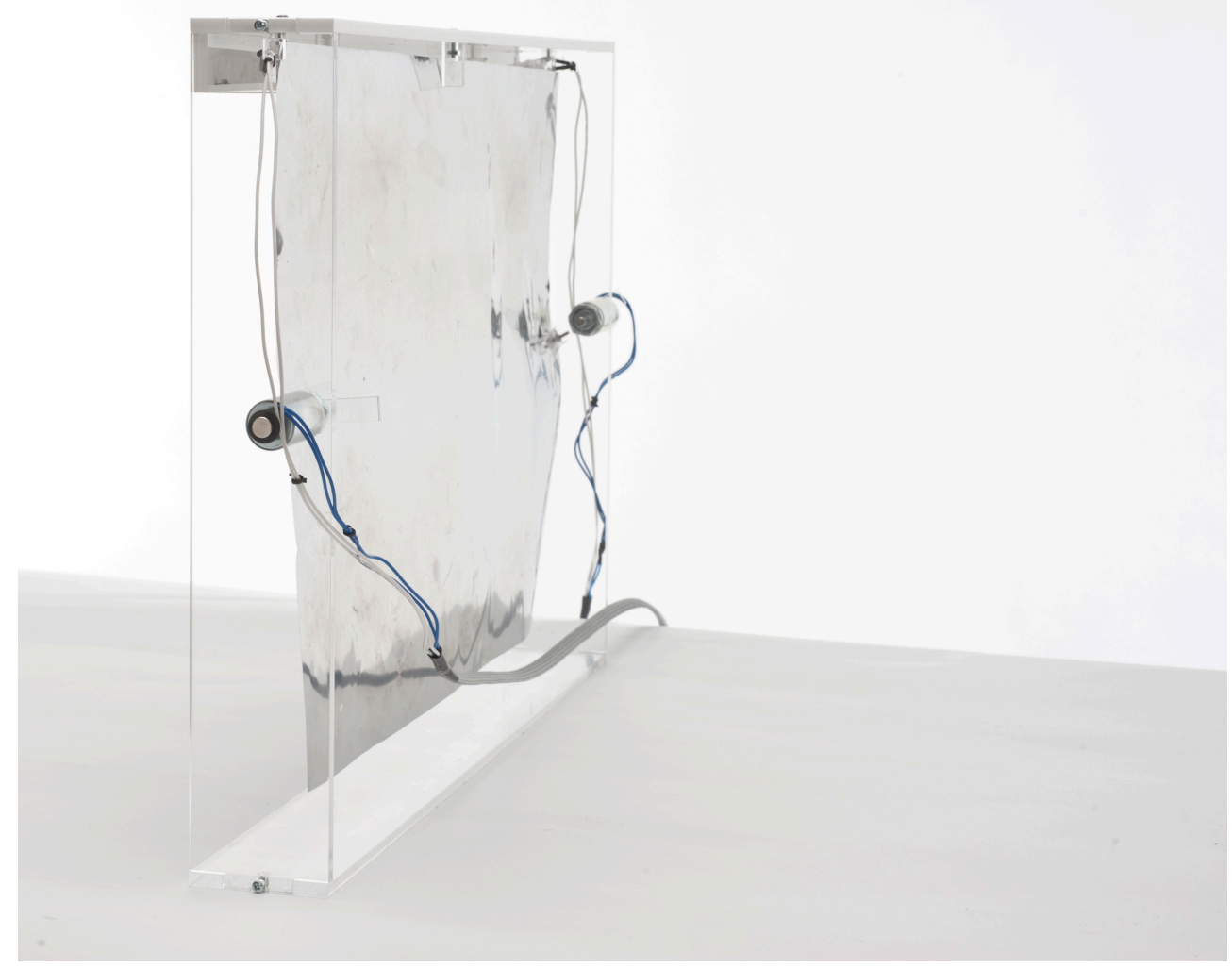

Figure 3-21. Rippler H-model (back view). 


\subsubsection{System Overview}

In both models, microcontroller programming is used to drive the solenoids. Using the same custom-designed driver board that was introduced in 3.2.2, the MIDI input data is transferred to the solenoids. Once the input messages are received, the solenoid puts the steel sheet into vibrating motion. Figure 3-22 provides a flow chart of this process.

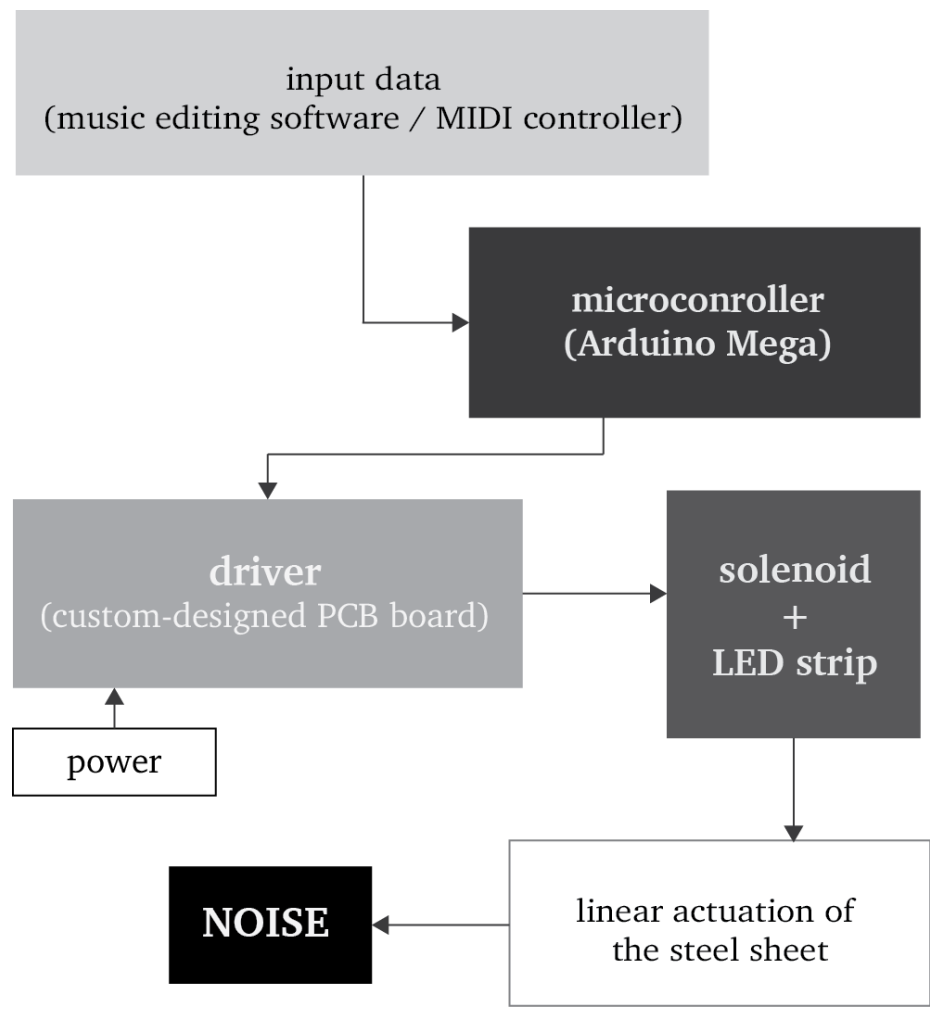

Figure 3-22. Rippler: system overview.

\subsubsection{Sound-generating Mechanism}

In the $\mathrm{V}$-model, once the solenoid receives a MIDI note-on message it pushes out and rapidly moves the sheet forward. When the MIDI note-off is received, the return spring of the solenoid pulls the solenoid's shaft and the sheet back to the rest position. This action creates a strike of noise: a decaying sound whose amplitude can be controlled by the MIDI velocity value of the MIDI message applied to the solenoid. Repeating this action leads to a series of strikes that, depending on the frequency of repetition, can produce a continuous sound. 
In the H-model, as the solenoids receive a MIDI note-on, the shafts move outwards and push the side edges of the sheet inwards, producing a strike of noise. Similarly, with the MIDI note-off, the return springs pull the shafts and the sheet back to their original position. Like the $\mathrm{V}$-model, repeating this cycle creates a sound that comprises the series of recurring noise strikes whose sonic characteristics are a function of the amplitude of the actuations (which is determined by the MIDI velocity value applied to the solenoids) and the rate of the repetition. The solenoids in the $\mathrm{H}$-model are independently addressed and driven by the corresponding MIDI note number. Therefore, in a continuous series of actuations different phasing effects can be created. This ranges from the constructive interference (when both solenoids are in the same phase), that is simultaneous contraction of the sheet from both edges, to the deconstructive interference (when solenoids are at the exact opposite phase), which results in moving the sheet sideways while keeping it straight (non-bent).
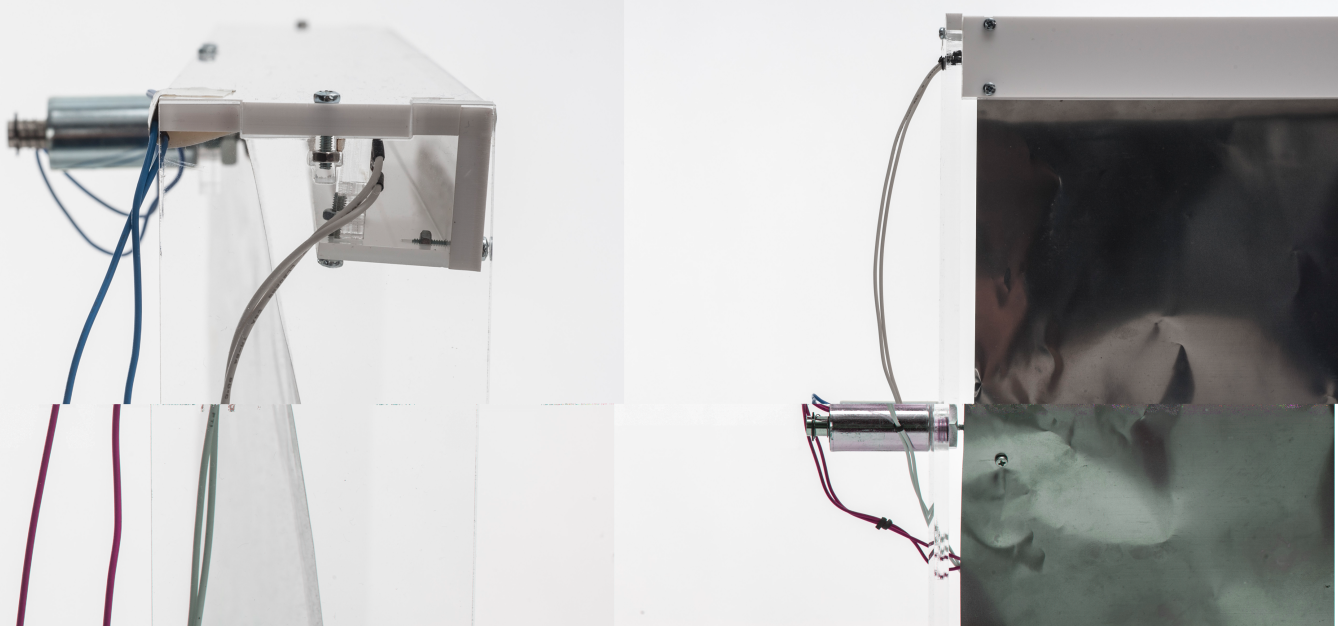

Figure 3-23. Rippler sound-generating mechanism: V-model (left), H-model (right).

\subsubsection{The Light Element}

In both models, the LED strips are controlled by the same signal as their associated solenoids. As the solenoid pushes out and in, all the LEDs on the corresponding strip turn on and off. The bright light of the individual LEDs that is diffused by the acrylic, illuminates the entire front and bottom panel of the LED tube and reflects on the steel, 
providing further visual feedback for its mechanical excitation (see Figure 3-24). In the H-model, the LED strip is divided into two individual halves, each corresponding to one solenoid. Therefore, the phasing and the interference patterns between the solenoids are visualised through the luminosity of the LED tube's front panel and its reflections on the sheet.

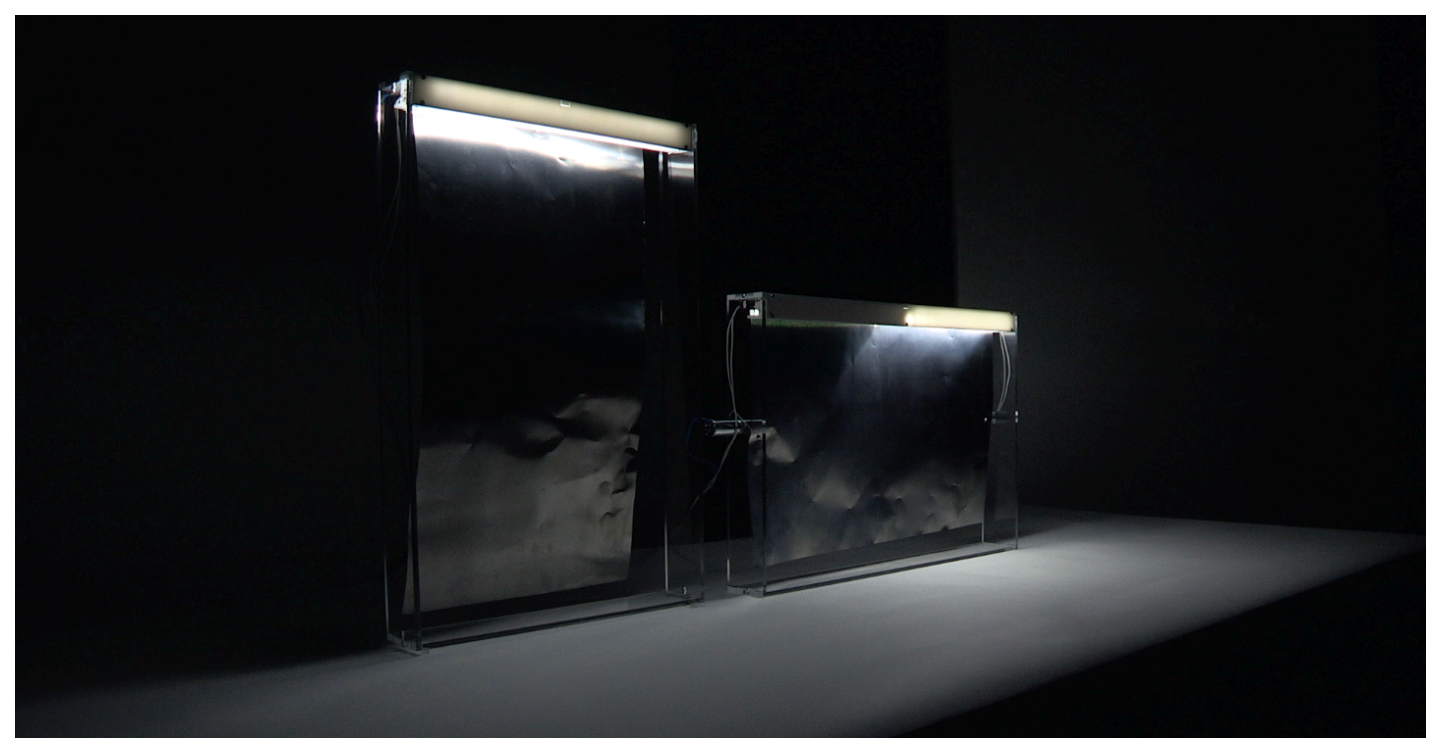

Figure 3-24. Rippler: the light element in both models.

\subsubsection{Evaluation}

In order to study the sonic characteristics of Rippler, a number of tests were conducted on recordings of both models. Recordings were collected by applying different MIDI velocity values to the solenoid to create an analysis of a number of key audio feature extractions, and - considering the decaying and percussive nature of the instrument's sonic output - time-dependent features.

In order to further investigate the sonic capabilities of the H-model (considering that it has two independent actuators), two separate sets of recordings for frequency-domain analysis at opposite actuation phase were conducted: one by simultaneous actuation of both solenoids, and one by actuation of a single solenoid. 


\subsubsection{Frequency-domain}

\section{Spectral Centroid and Spectral Roll-off}

Considering that Rippler's sound is produced completely mechanically, the output results for the same input values might slightly vary in every trial. Therefore, for each model, a set of five recordings was made for each MIDI velocity and the analysis was computed on the average of the five values. The input values ranged from MIDI velocity 127 down to MIDI velocity 57 every 5 steps (as lower values were incapable of exciting the sheet in an audible manner), and the feature extractions were calculated with the following specifications (similar to Rasper):

- Sample Rate: 44100 sample/s

- Window Function: Hanning

- Window Size: 1024 samples.

For each input value, the solenoid strikes were generated using a $64^{\text {th }}$-note long MIDI note at 120BPM.

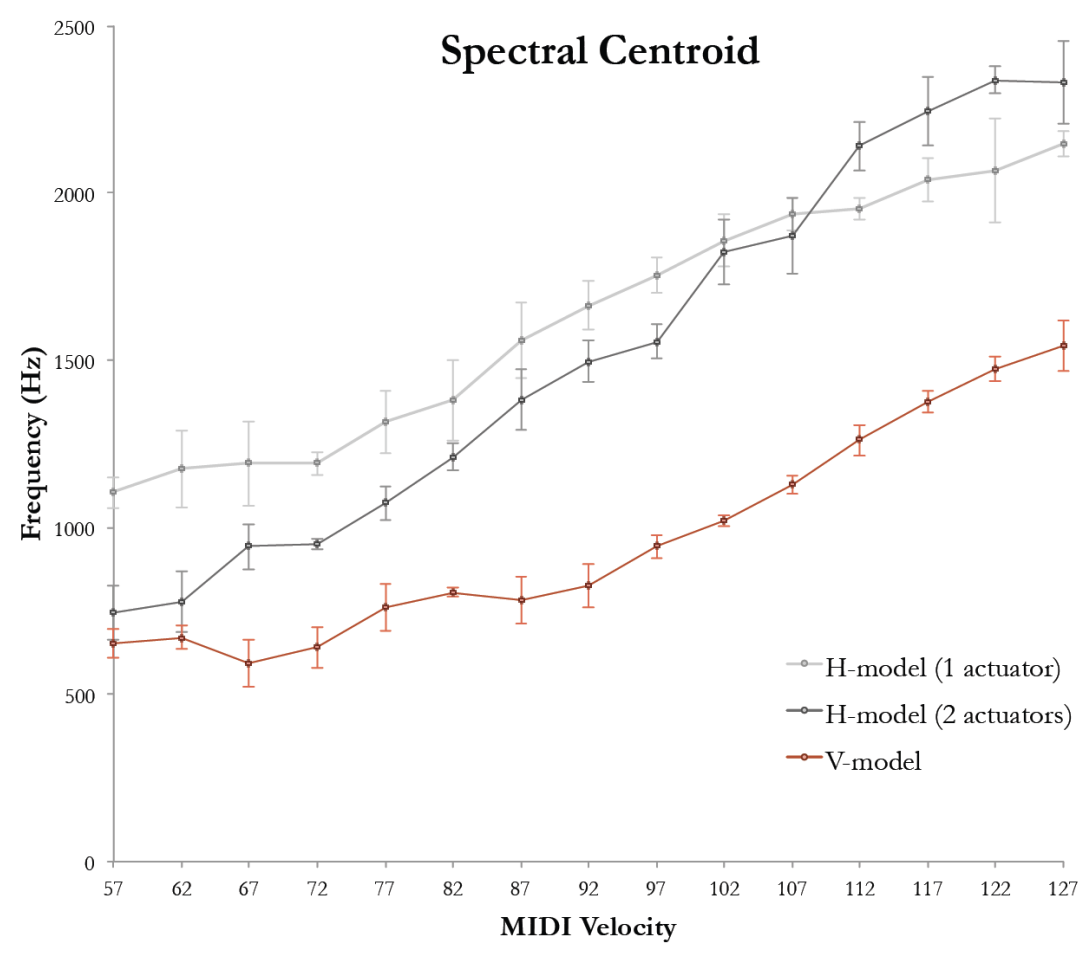

Figure 3-25. Rippler: spectral centroid. 
The concentration of the centre of mass of the audio signal for all three recordings is shown in Figure 3-25. According to this figure, this centre frequency is greater for stronger excitations that are created by higher MIDI velocities. Additionally, the centre frequency is notably higher for the H-model, which can be perceived as a brighter sonic output for this model. It can also be noticed that, when sweeping the input values from lowest to highest in the H-model, a moderately wider dynamic range is achievable with simultaneous actuation of both solenoids. Respectively similar conclusions are extracted from the spectral roll-off plots below, where frequencies marking 50 percent of the power distribution of the spectrum are exhibited. However, the notably increasing value of the standard deviation for greater MIDI velocities in the V-model shows the instrument's more chaotic behaviour for high-amplitude excitations.

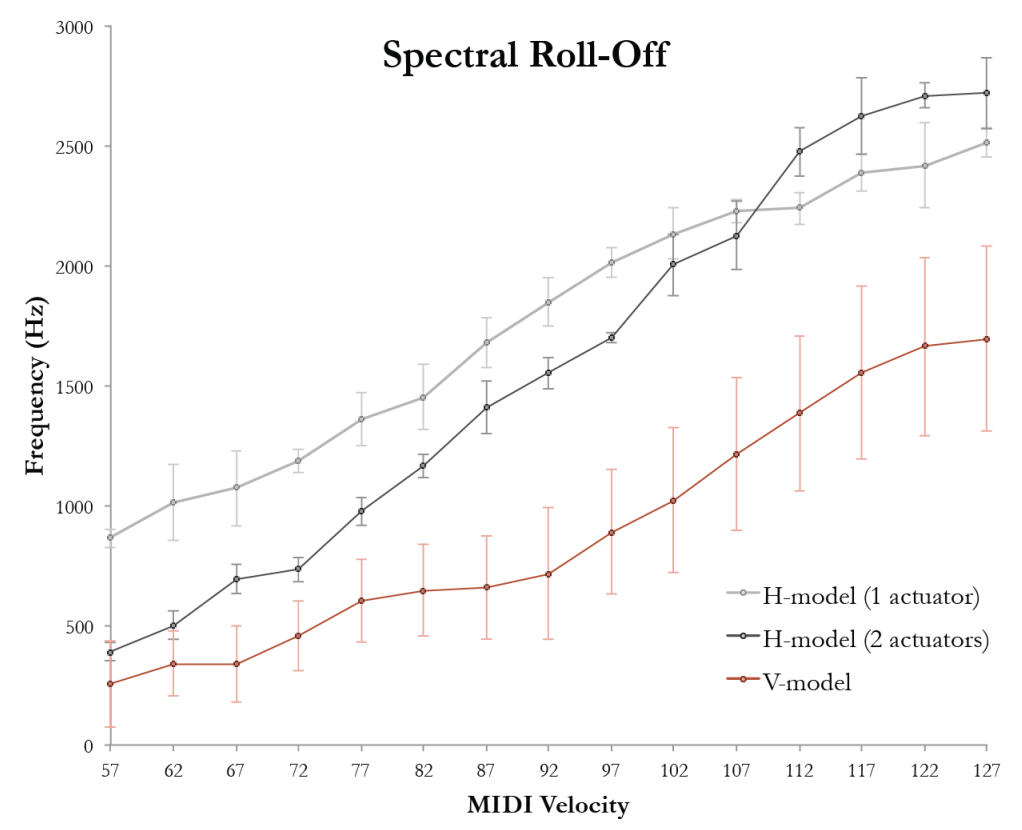

Figure 3-26. Rippler: spectral roll-off.

\section{Zero-crossing and Spectral Flux}

If interpreted as the instrument's subtle "sense of pitch" and general frequency response, the zero-crossing rates provided in Figure 3-27 demonstrate the comparatively wider frequency range and higher response of the $\mathrm{H}$-model. The almost chaotic behaviour of the flux of change of the audio signal as the input values change, in addition to the 
significant standard deviation values provided in Figure 3-28, discloses Rippler's noisy sonic essence. According to this figure, although the trend is for flux to increase with MIDI velocity, the 'MIDI velocity-timbre' (or 'input-output') relationship does not follow a linear behaviour with smaller changes in the input value.

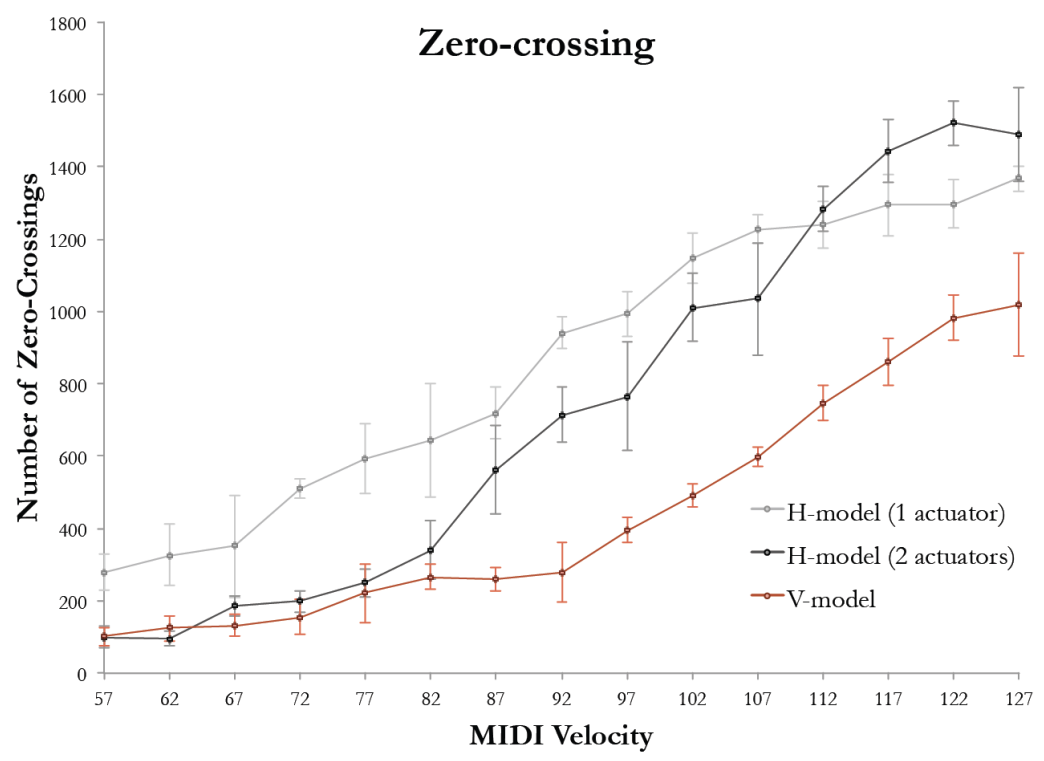

Figure 3-27. Rippler: zero-crossing.

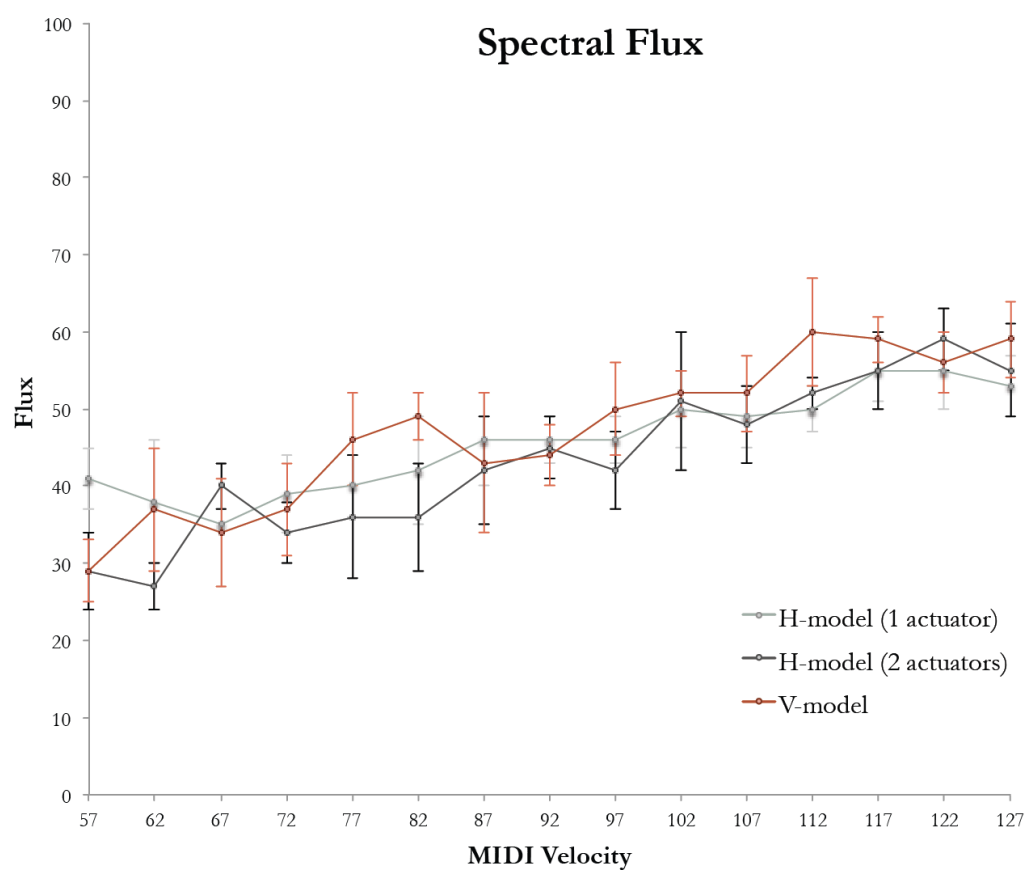

Figure 3-28. Rippler: spectral flux. 


\subsubsection{Time Domain}

The percussive and decaying nature of Rippler's sonic output is exhibited in Figure 3-29. 1 -second long recordings of a single strike generated by a MIDI velocity value of 127 for both models are provided in waveform format. The top two waveforms demonstrate recordings of the H-model in (a) single actuation mode and (b) double actuation mode. Comparing the two waveforms, the substantially shorter attack time in single actuation mode and the relatively longer decay time in double actuation mode denote a timbral difference between the two modes of actuation. This timbral difference can be also noticed in the V-model waveform (c) with its considerably longer attack time, as well as the slightly longer decay time. Moreover, latencies of approximately $30 \mathrm{~ms}$ for single actuation, and $60 \mathrm{~ms}$ for double actuation can be noticed in the H-model. Considering that all three strikes are generated by the same input value, the latency difference observed in the three waveforms leads to the conclusion that this latency is primarily dependent on the mechanics of actuation and the mode of excitation.

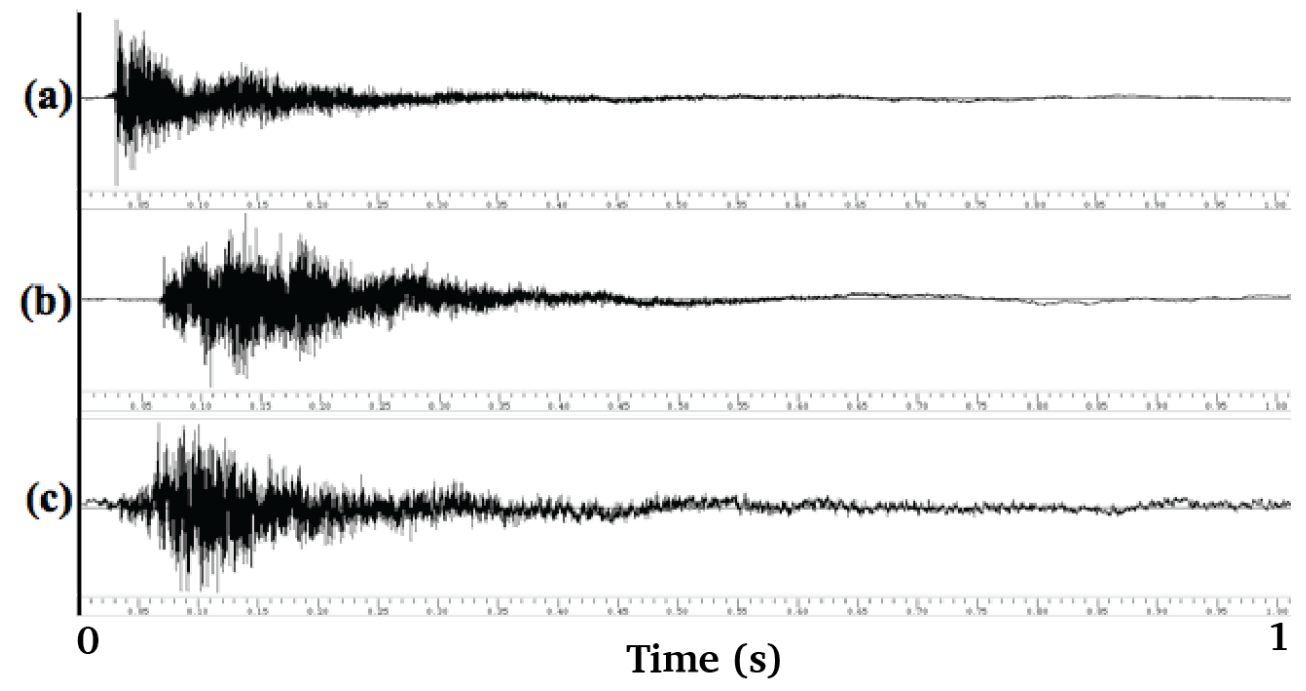

Figure 3-29. Rippler: waveform representation of recordings for a single strike: (a) H-model, single actuation (b) H-model, double actuation (c) V-model.

The percussiveness of Rippler's sound naturally calls for its use in rhythmic contexts. With this in mind, in addition to the latency phenomenon discussed above, it is important to probe the instrument's capability and responsiveness in terms of speed in 
order to further evaluate its rhythmic abilities. Therefore, a series of recordings were made for each model, in which the sheet is excited by consecutive $16^{\text {th }}$-note long MIDI notes, with a MIDI velocity of 127 , over a period of two seconds. For each recording, the speed of actuation was changed, ranging from 100BPPM to 700BPM, in steps of $100 .^{18}$ As the speed goes higher and the frequency of actuation increases and falls into the audible range, the discretely perceived strikes turn into a rather continuous hum. During the experiment, it was noticed that this timbral change takes place at a more dramatic rate once the speed passes 600BPM. This can be explained as follows:

$$
\begin{aligned}
& 600 \mathrm{BPM}=10 \mathrm{BPS} \\
& 16^{\text {th }} \text { strikes }=4 \text { strikes per beat } \\
& (1) \text { and }(2) \Rightarrow 10 \mathrm{BPS}=40 \mathrm{~Hz}
\end{aligned}
$$

Somewhere between 600BPM and 700BPM, the dramatic rate of timbral change peaks, and at a BPM of approximately 700 (approximate actuation frequency of $47 \mathrm{~Hz}$ ), where the current switching in the solenoid becomes so quick that the shaft cannot move, the output is inaudible.

Considering that at higher speeds and after a certain threshold, the attack and decay times are much longer than the interval between the strikes, the recorded waveforms appear to be noisy at all times. As a result, it would be extremely difficult to visibly perceive these speed-related performance features and timbral changes in the waveform representation. Therefore, the results provided in Figure 3-30 are represented by the average number of zero-crossings over two seconds of recording for each input value. Based on the general behavioural similarity of the two models suggested by this figure, it can be concluded that these speed-related features are primarily a function of the performative characteristics and capacities of the actuators, and therefore, independent of the style of actuation.

\footnotetext{
${ }^{18}$ Except for the interval marked by 600BPM and 700BPM, in which the recordings are made at every 10 steps.
} 


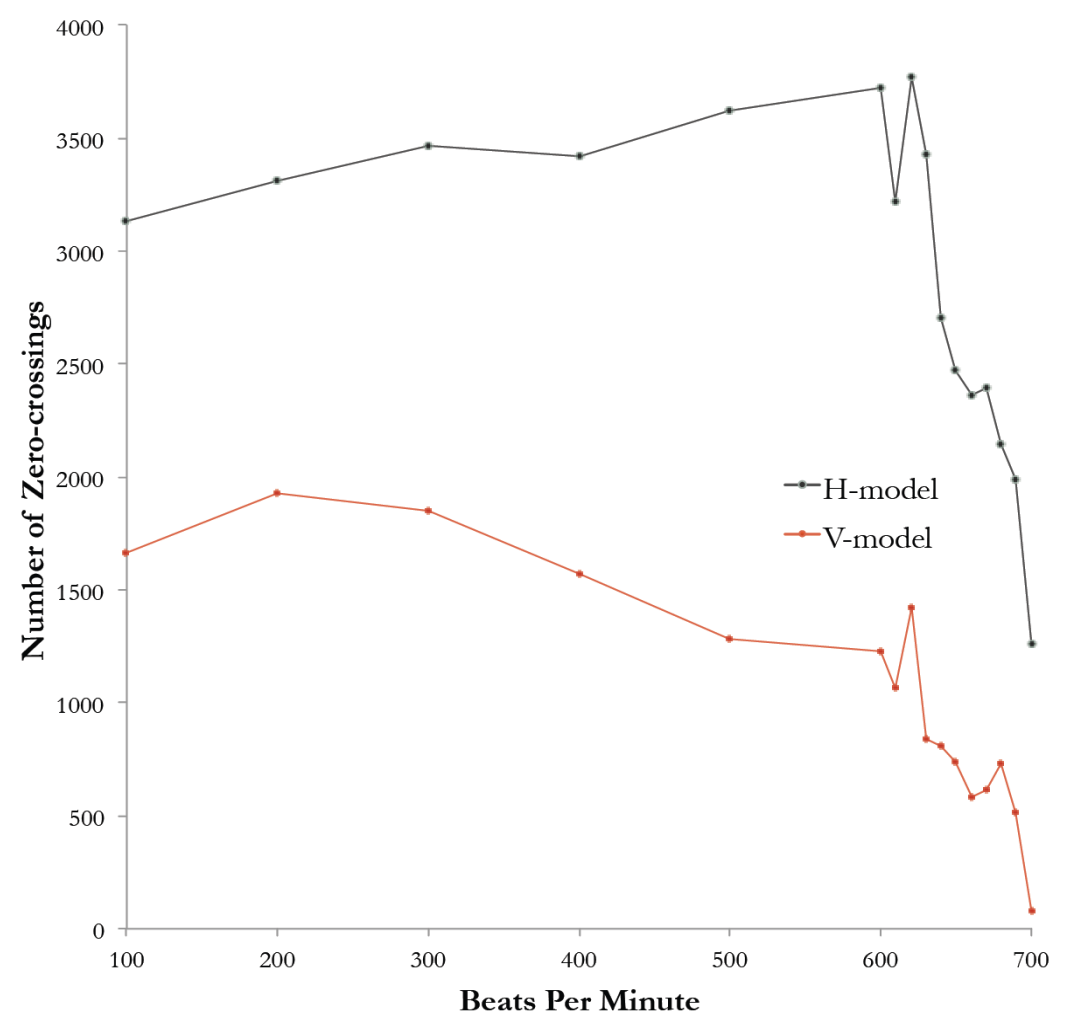

Figure 3-30. Rippler's Speed Performance in both models.

\subsubsection{Summary}

Acoustically, Rippler generates noise rather than musical tones. In terms of frequency, Rippler's sonic output primarily lies in the low and low-mid range (based on the classification described in Error! Reference source not found.). The instrument produces percussive sonic output and can be driven at high frequencies. The percussive sonic output enjoys only limited timbral variety based on the input values. Additional timbral variety can be achieved by using different models of the instrument, or different modes of actuation within the H-model. The timbral variety in the output does not correlate to the input in a completely linear and predictable manner. The process of receiving the input messages from the software and their physical implementation on the output is delayed by a short latency. Where rhythmic synchrony is concerned, the actuation latency of Rippler should be taken into account.

\subsection{Mutor}




\subsection{Mutor}

Mutor is the third and last sound-sculpture in the series of mechatronic noise-intoners developed over the course of this research. As mentioned in the beginning of section 3.3, the actuation noise of the mechatronic components (that is the solenoid and the motor) in Rasper inspired the design of the succeeding sound-sculptures. Accordingly, if Rippler's sound-generating mechanism highlights the solenoid actuation noise by transducing it through a sheet of steel, Mutor directly focuses on the actuation noise of the DC motor.

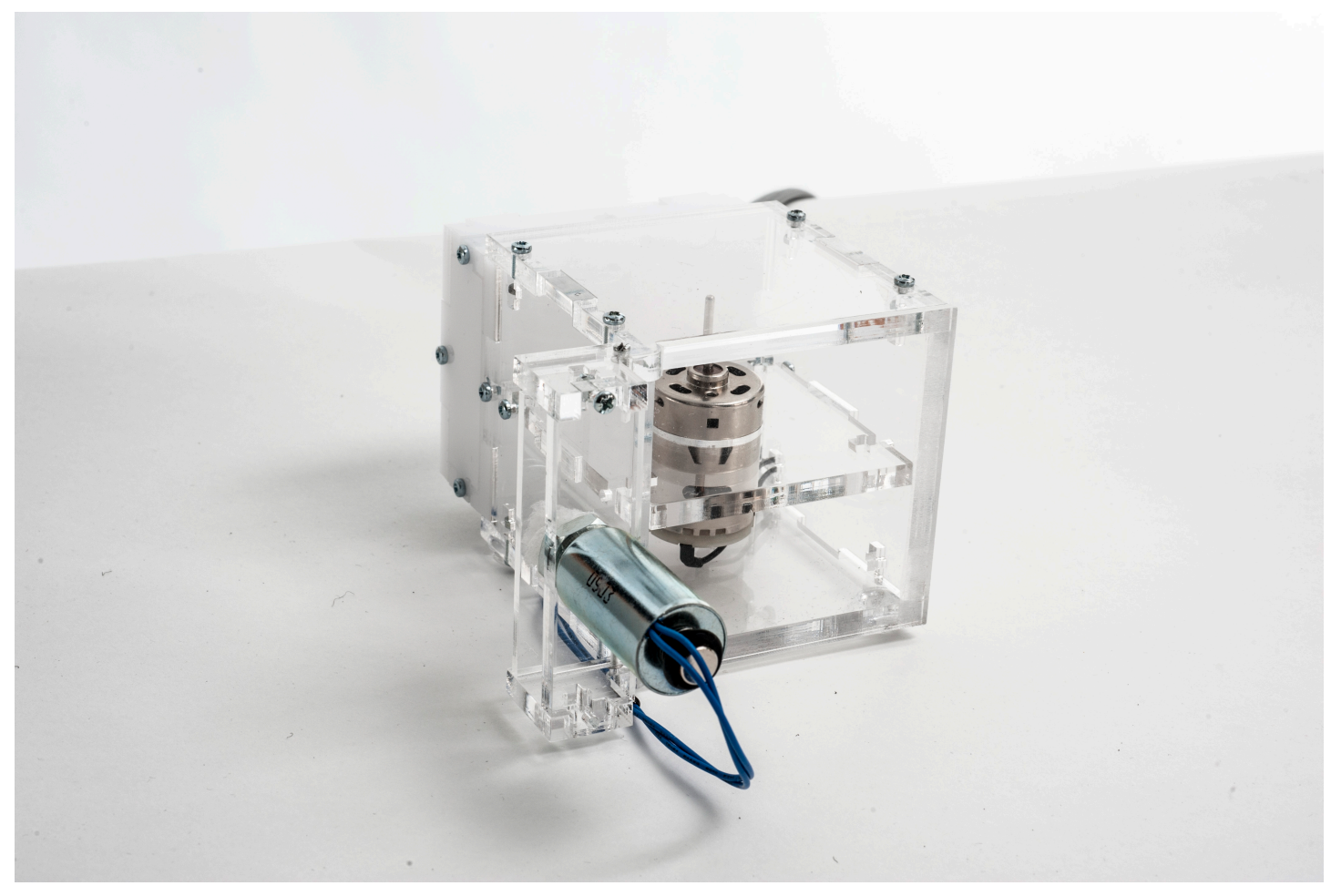

Figure 3-31. Mutor.

\subsubsection{Design}

Mutor comprises a $12 \mathrm{~V}$ DC motor placed in a transparent cubic box with a pivoting door, and a smaller white acrylic box enclosing a short strip of LEDs that is attached to the back of the main box. The motor is fixed in the centre of the cube, using a transparent bracket. A $12 \mathrm{~V}$ push solenoid is mounted on the side of the cube, using 
another transparent bracket, and is attached to the pivoting door in order to open and close it. For both visual and sonic coherence and consistency, the motor and the solenoid are of the same type used by the previous sound-sculptures.

The cube and the brackets are designed using CAD technology. First, 3D models of the mechatronic component were drafted in SolidWorks. Then, using CAM technology, the box and the bracket are laser-cut. All parts are cut from $6 \mathrm{~mm}$ thick acrylic, with the exception of the pivoting door, which is cut out of $3 \mathrm{~mm}$ thick acrylic in order to facilitate its opening motion. The LED box is cut out of frosted white acrylic in order to diffuse the individual LED lights and illuminate the entire box homogeneously. The LED strip contains three bright cool white LEDs and is glued on the inside of the LED box, with the LEDs facing forward. The design sketch, parts, and 3D model of Mutor are provided in the figures below.

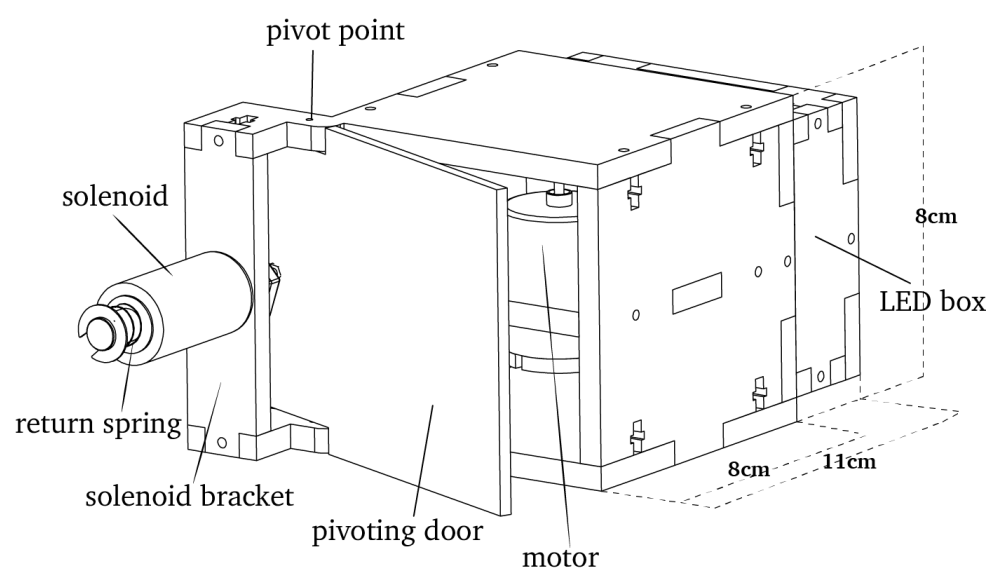

Figure 3-32. Mutor: design sketch and parts.

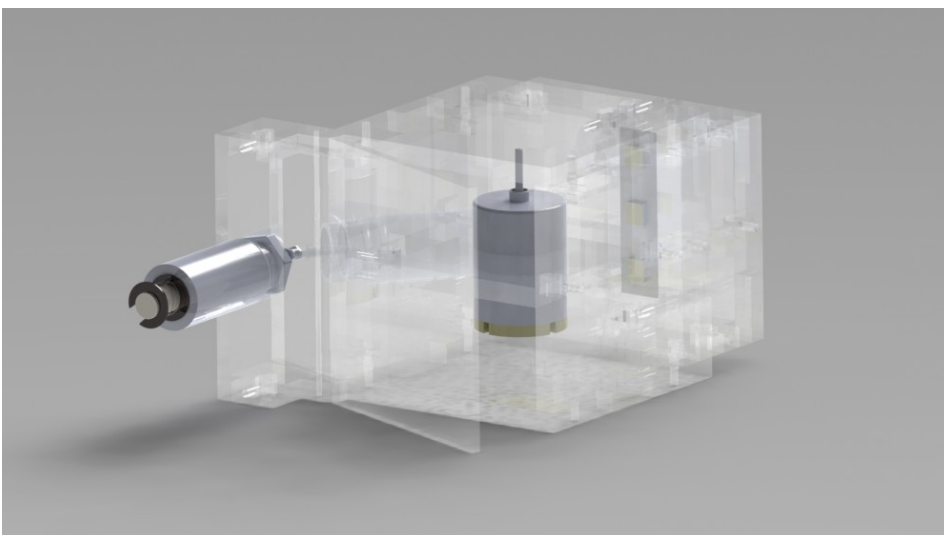

Figure 3-33. Mutor: 3D model. 


\subsubsection{System Overview}

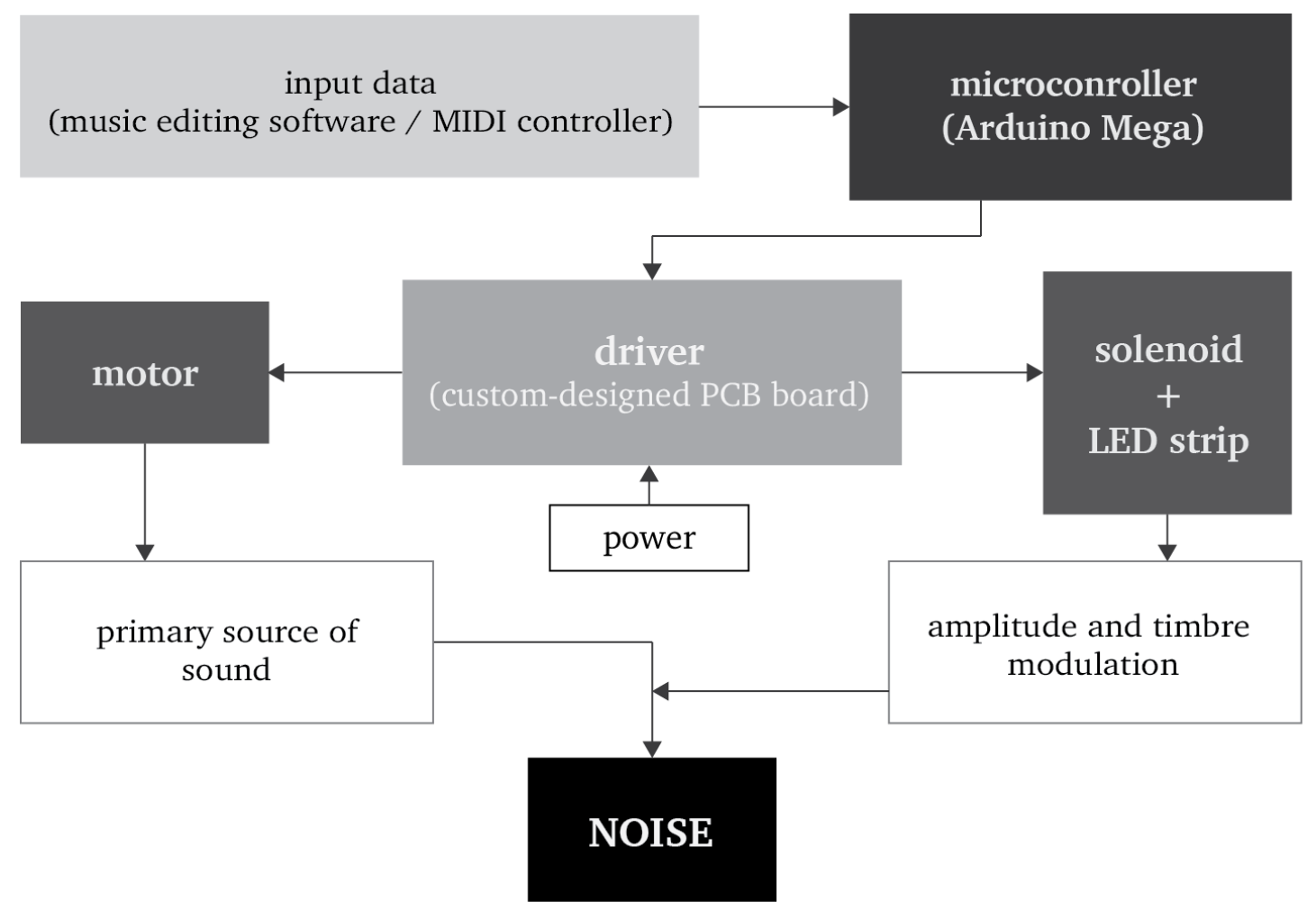

Figure 3-34. Mutor: system overview.

Similar to Rasper and Rippler, Mutor takes MIDI messages as input, and is driven by the same custom-designed driver board employed by the previous sound-sculptures. Separate input messages for the motor and the solenoid are addressed by different MIDI note numbers, and MIDI velocity values correspond to the amplitude of actuation. Figure 3-34 illustrates a flow chart of the system overview.

\subsubsection{Sound-generating Mechanism}

The primary source of sound in Mutor is the actuation noise of the DC motor. The solenoid is used to modulate this sound in terms of timbre and amplitude, by opening and closing the enclosure in which the motor is placed, that is the acrylic box.

Once the driver-board receives the input MIDI messages, MIDI velocity values are converted into voltage and sent to the designated component. The motor inputs correspond to the speed of rotation, and are used to produce different buzzing tones in a 
continuous manner. Different MIDI velocity values sent to the solenoid correspond to the solenoid's stroke length. Greater stroke lengths cause wider angular displacement of the pivoting door, and consequently, greater timbre and amplitude modulation. When there is no input for the solenoid and the shaft is in the rest position, the door is shut and the motor's sound is in the 'muted' state. Once the solenoid receives the input, based on the corresponding MIDI velocity value of the message, it opens the pivoting door by pushing its outer edge, placing the motor's sound in the 'unmuted' state. With the MIDI note-off message, the current in the solenoid is switched off and the return spring brings the shaft back to the rest position, returning the instrument to its muted state.

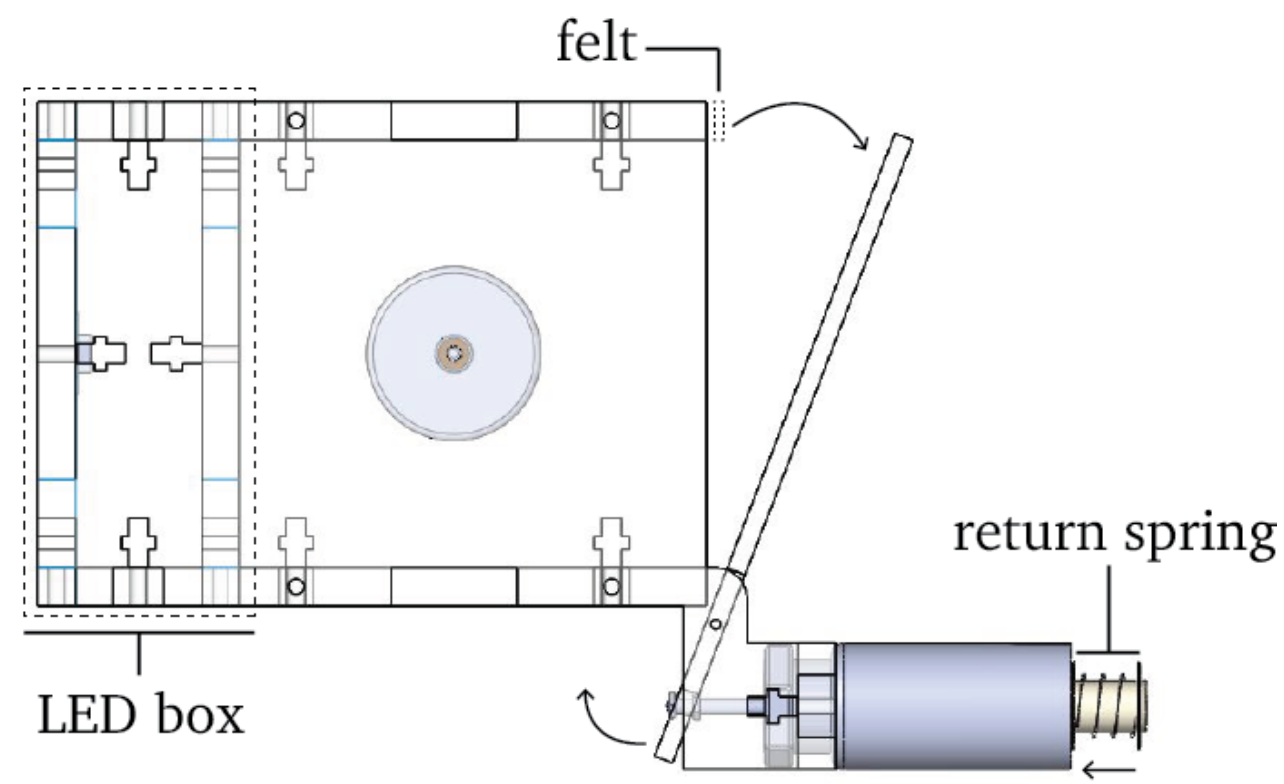

Figure 3-35. Mutor: sound-generating mechanism (top view).

Although the motor's sound is the primary and dominant sonic output of Mutor, the sound of the door shutting introduces a percussive flavour to the aural output, emphasising its rhythmic element. In order to dampen this percussive effect, and to preserve the dominance of the motor's sound, a thin narrow strip of felt is glued on the inner side of the edge of the pivoting door, where it makes contact with the edge of the box (see Figure 3-35). 


\subsubsection{The Light Element}

The LED strip comprises three bright fluorescent LEDs and is mounted on the inner side of the LED box (see Figure 3-36). The choice of light colour was made in aesthetic consistency with the previous sound-sculptures. The LED box which is made out of $6 \mathrm{~mm}$ thick diffused white acrylic, is attached to the back of the main cube. The same input that triggers the solenoid is used to drive the LEDs. Therefore, when the solenoid receives an input and puts the instrument in the unmuted state, the LEDs illuminate the LED box, which then brightens the entire cube. This correlation is chosen in order to further strengthen the rhythmic perception of the timbral modulation.
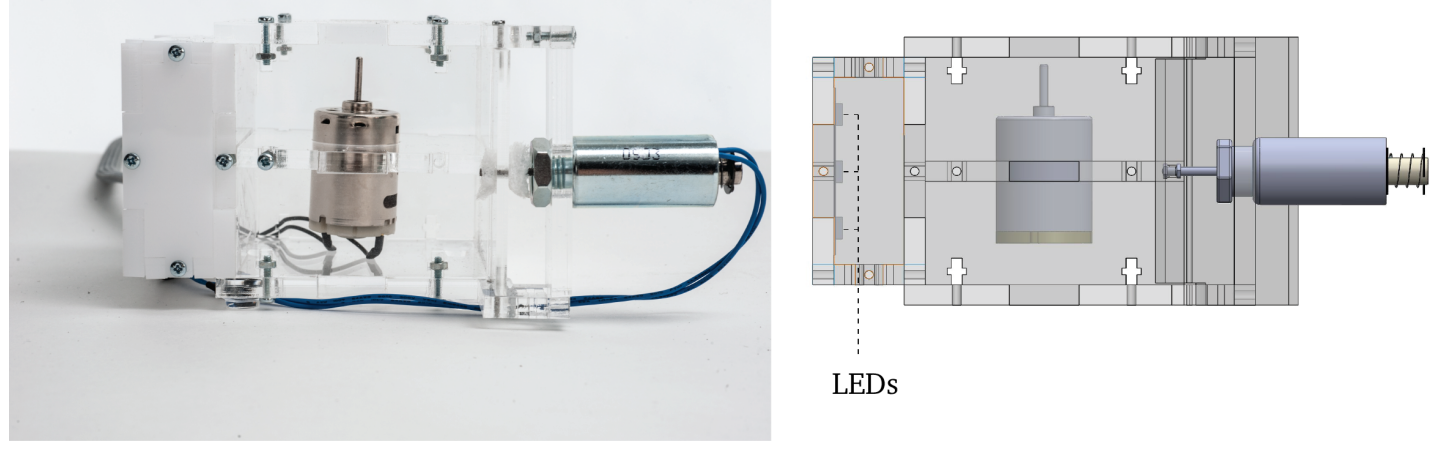

Figure 3-36. Mutor: light component.

\subsubsection{Evaluation}

In order to evaluate some sonic and performative characteristics of Mutor, a number of tests were carried out on recordings of the instrument, in both muted and unmuted states. This section provides the information extracted from these tests, regarding both frequency- and time-domain features of Mutor.

\subsubsection{Frequency-domain}

In order to make an assessment of the frequency characteristics of Mutor, a number of key feature extraction tests were carried out on recordings of the instrument in both states (muted and unmuted). One separate series of recordings is made for each state. For the muted state, no input messages are sent to the solenoid in order to keep the pivoting door of the box closed, whereas the unmuted recordings are made by sending a 
MIDI message with MIDI velocity of 127 to the solenoid, opening the pivoting door to its widest open angular position. For each state, a series of recordings are made by applying different MIDI velocity values to the motor, ranging from MIDI velocity 127 all the way down to MIDI velocity 7, every 10 steps, that is a total of 13 different 1 second long recordings were made for each state. Due to the inconsistency and noisiness of the sound of a DC motor, the feature extractions are obtained from average FFT data of the recordings with the following specifications (as per Rasper and Rippler):

- Sample Rate: 44100 sample/s

- Window Function: Hanning

- Window Size: 1024 samples.

Again, feature extractions for 1-second long recordings are calculated as the average of approximately 43 samples $(44100 / 1024 \cong 43)$.

\section{Spectral Centroid and Spectral Roll-off}

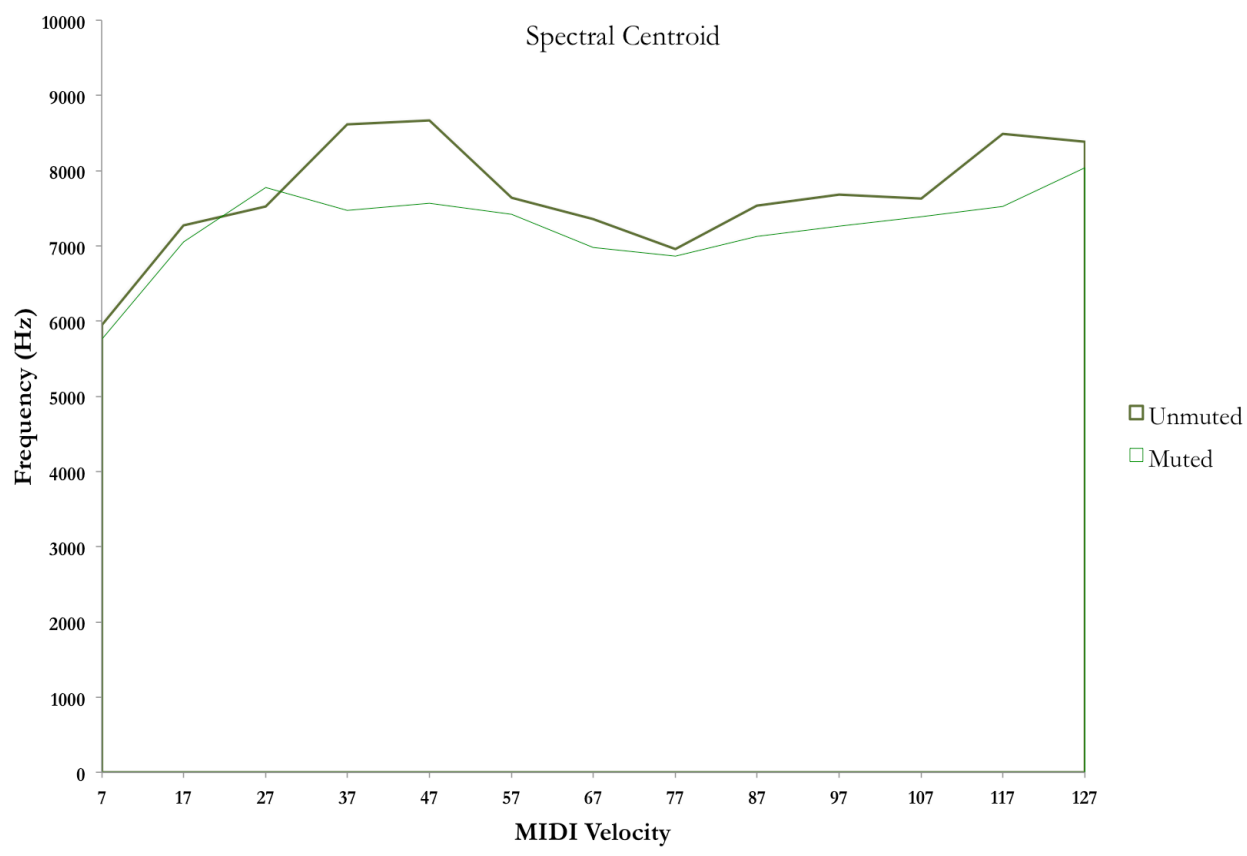

Figure 3-37. Mutor: spectral centroid. 
Figure 3-37 shows that the centre of mass of the audio signal in both muted and unmuted states, for the majority of input values, lies in the relatively narrow frequency band of $7 \mathrm{kHz}$ to $9 \mathrm{kHz}$. The respectively higher centre of the mass in the unmuted graph represents the timbral difference between the two states: a phenomenon that is more clearly notable in Figure 3-38. In this graph, frequencies marking 50 percent of the power distribution of the spectrum are significantly higher in the unmuted state.

The information extracted from these two charts verifies that, when putting Mutor in the unmuted state, higher frequencies, overtones, and partials enter the sonic output, making it perceptually brighter. This is due to the fact that the acrylic box acts as a lowpass filter. Therefore, with the door closed, some higher frequencies are eliminated from the output.

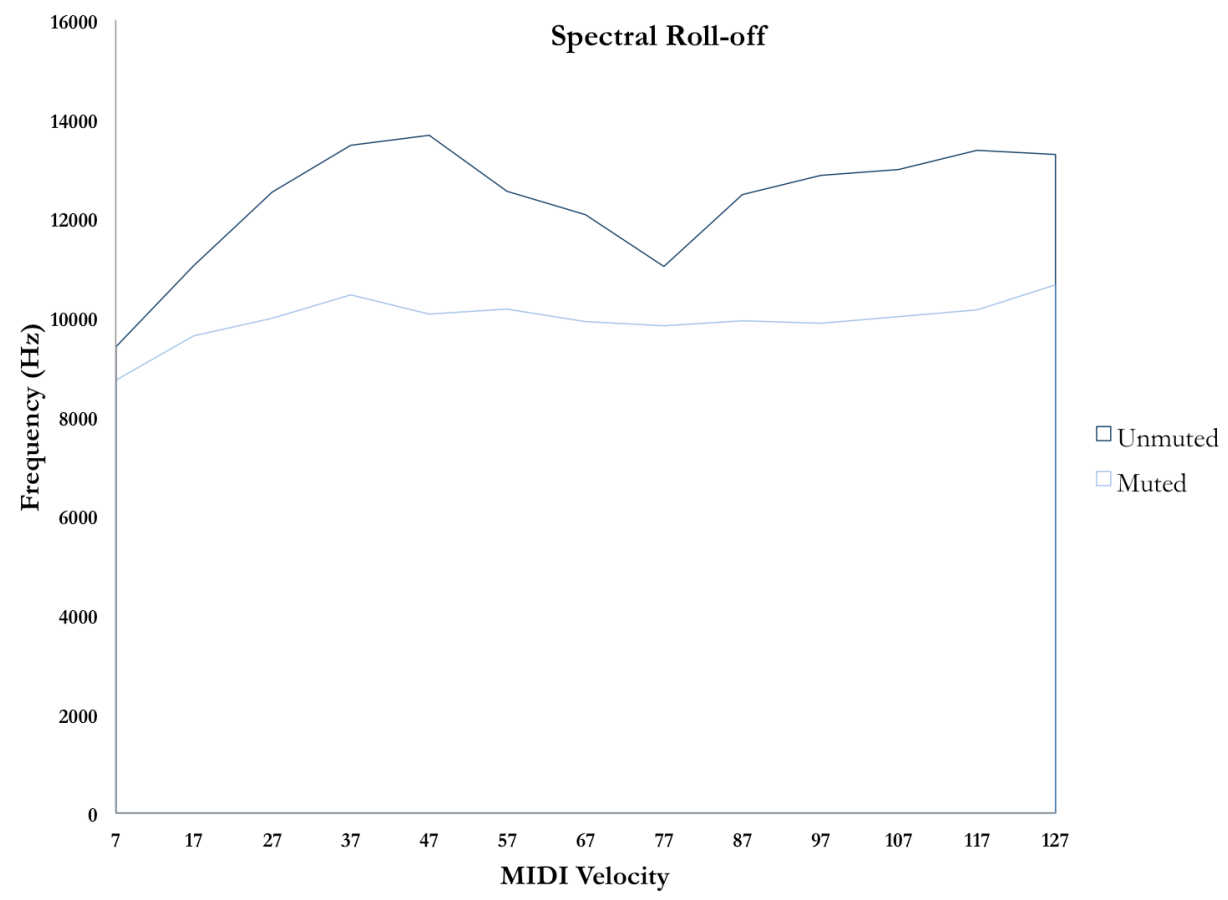

Figure 3-38. Mutor: spectral roll-off.

\section{Zero-crossing}

The highly wavering behaviour of the (scaled) number of zero-crossings in both muted and unmuted states can be viewed in Figure 3-39. Throughout the input range, there does not seem to be any form of consistency or linearity. The chaotic and inconsistent 
correlation of the input and output in this graph further clarifies Mutor's noisy sonic quality. As a result, various motor inputs lead to a timbral and frequency variation in the output, but in doing so, they do not follow a particularly predictable pattern.

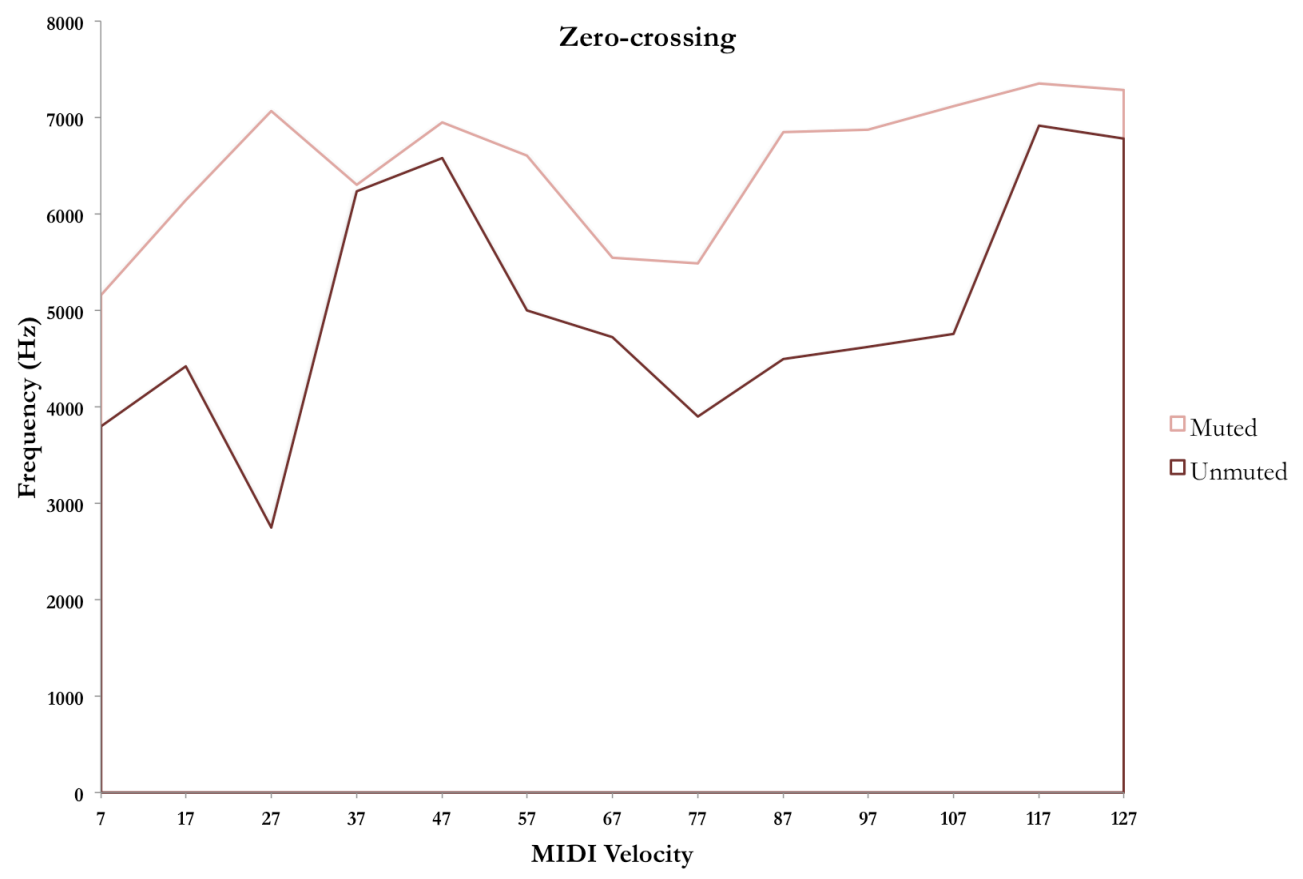

Figure 3-39. Mutor: zero-crossing.

\subsubsection{Time Domain}

Figure 3-40 provides the visualisations of a 1-bar long pattern played by Mutor in MIDI, waveform, and spectrum representations. The pattern is comprised of four $8^{\text {th }}$-note long MIDI input messages with a velocity of 127 sent to the solenoid (at 120BPM), putting the instrument in the unmuted state. Each input message is followed by an $8^{\text {th }}$-note long period of no input, during which the door is set back to the closed position and the instrument is in the muted state. Throughout the entire pattern, the speed of the motor is kept constant by sending a 1-bar long MIDI message with the velocity of 127 to the motor. Figure 3-40 (a) shows the MIDI notes used to trigger the solenoid and run the motor, (b) exhibits the waveform modulation over a period of 2 seconds (a period of one bar at 120BPM), and (c) provides the spectrum distribution of the recordings of the instrument, while playing the pattern. 
(c)

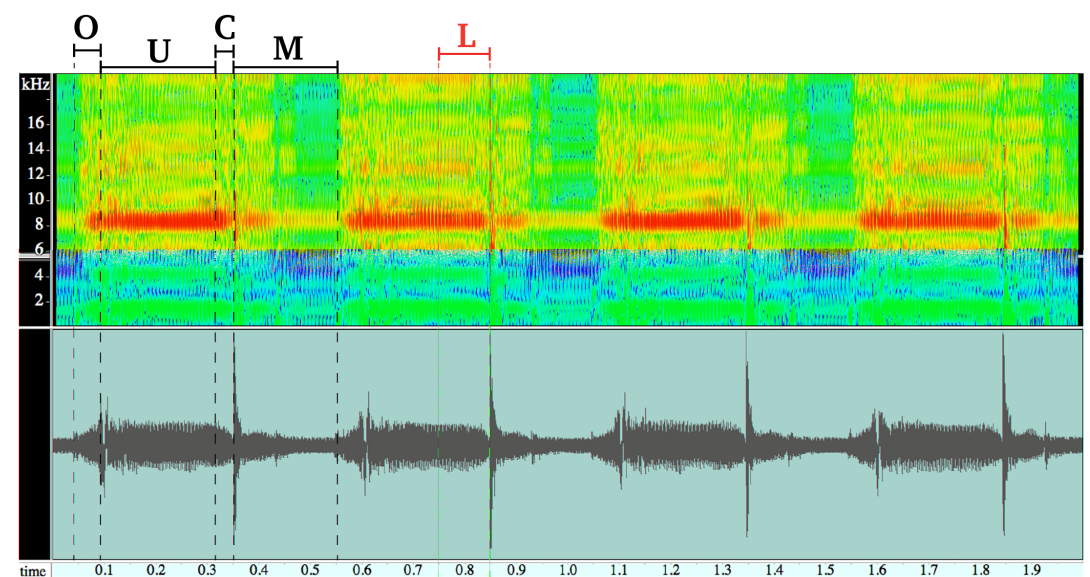

(a)

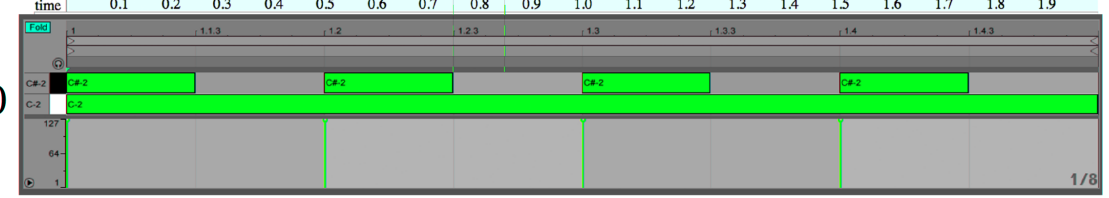

Figure 3-40. Visualisation of a 1-bar long pattern played by Mutor: (a) MIDI, (b) waveform, (c) spectrum.

The time intervals constituting the pattern are separated and marked with labels:

- O: sending the note-on message and opening the door to its widest angle,

- $\mathbf{U}$ : the unmuted state interval,

- C: sending the note-off message and closing the door,

- $\mathbf{M}$ : the muted state interval,

- $\quad \mathbf{L}$ : the latency between triggering the input from the software and its mechanical implication at the output.

The latency $(\mathrm{L})$ has been represented as the time interval between receiving the note-off message and the actual shutting of the box's door. This latency of approximately $100 \mathrm{~ms}$ is the time that is required for the solenoid's return spring to decompress and close the door, and is of course unavoidable. Nevertheless, given the fact that the most distinctive percussive element of Mutor that emboldens the rhythmic aspect of its sonic output is the sound of the door shutting, this latency should be carefully accounted for, especially in a context where rhythmic synchrony is of importance. The relatively consistent modulations in the shapes of the waveform and the spectrum in all four strikes show that with constant inputs, relatively constant outputs can be expected from Mutor. 


\subsubsection{Summary}

Acoustically, Mutor generates noise rather than musical tones. The output noise primarily lies in the mid to high-mid frequency range. Mutor's sonic output has both continuous and percussive characteristics. The continuous output is timbrally modified by the motor inputs, and rhythmically modified by the solenoid inputs. The percussive output correlates to the solenoid inputs. Actuation latency of the instrument should be accounted for where synchrony is concerned.

\subsection{Collective Evaluation}

In the previous three sections, various design features and sonic characteristics of the individual sound-sculptures were discussed. As demosntrated throughout this chapter, the same design and aesthetic principles are followed and preserved in the design and construction of all instruments. This consistency can be clearly observed in choice of the material and components, as well as the visual form and structure of the soundsculptures.

In order to provide a more comprehensive evaluation of all three instruments as a system, it would be useful to make a comparison of their sonic capabilities and characteristics that were individually examined in each section. Therefore, this section briefly contrasts some of these sonic features and characteristics to provide a general image of the sound-sculptures as a system.

\subsubsection{Relative Frequency Range}

Figure 3-41 compares the spectral centroid graphs of Rasper, Mutor, and the two different models of Rippler. Due to the greater dynamic range of the double-actuation mode in Rippler's H-model, the graph marked with "Rippler H" corresponds to the data from double-actuation mode recordings. The data provided in this figure are extracted from the individual analyses and evaluations of the instruments presented earlier in this chapter. This selected data represents the output for every 10 MIDI velocity values in 
the functional input range of each instrument. This is only representative of the centre of the mass of the audio signal, and does not provide a detailed and thorough comparative analysis of frequency characteristics of all the instruments combined. However, the completely spread-out and isolated centroid range of the individual sound-sculptures in the frequency spectrum can be interpreted as their distinctive timbral and frequency scope, from the low and low-mid, to the mid, and high frequency ranges. Accordingly, although the frequency and timbre range within each instrument is not particularly extensive, the sonic output of each instrument contributes to a different region in the frequency spectrum.

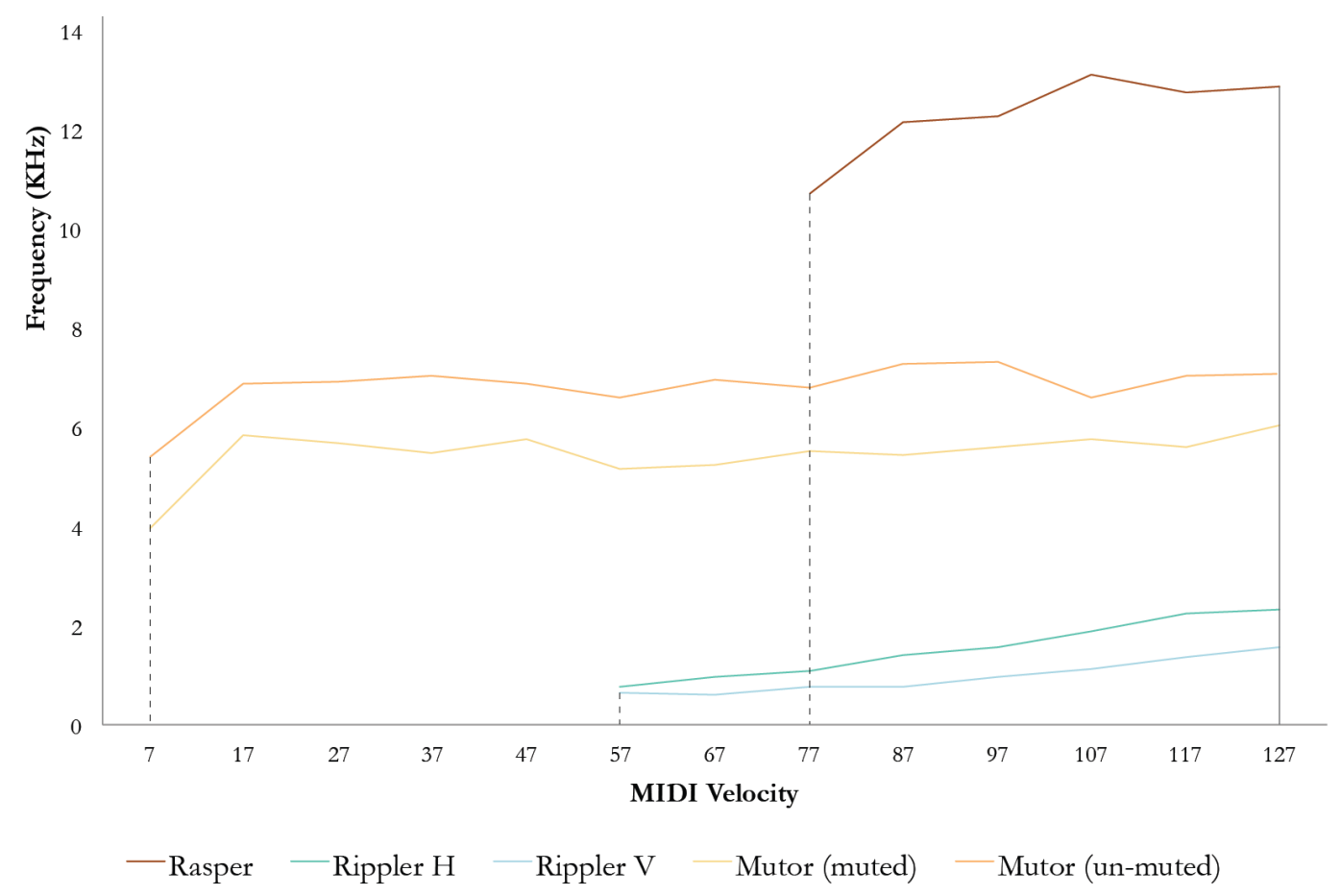

Figure 3-41. Spectral centroid of Rasper, Rippler, and Mutor compared.

\subsubsection{Relative Loudness}

In another series of tests, the maximum sound intensity of each sound-sculpture was measured in order to make a relative loudness comparison. For each sound-sculpture, the maximum loudness was measured by the peak function of a digital sound-level meter placed one metre away from the instrument. In order to expand the comparison to different sonic qualities of each sound-sculpture, especially for different rhythmic 
contexts, each test is carried out in three different input scenarios: once for a one-off quarter-note input message, once for a looped sequence of $16^{\text {th }}$-note long input messages, and once for a looped sequence of $32^{\text {nd }}$-note long input messages. In all three scenarios, the inputs were generated at 120BPM, with the maximum MIDI velocity value applied to all components. For each sound-sculpture, each test was repeated five times. As a result, the data represented in Figure 3-42 are the average of five trials. Considering the purpose of the test is to get a general idea of the relative loudness of the instruments as perceived by ears, the A-weighting scale was used [53]. The average maximum noise level of the room during the test was measured as $47 \mathrm{dBA}$.

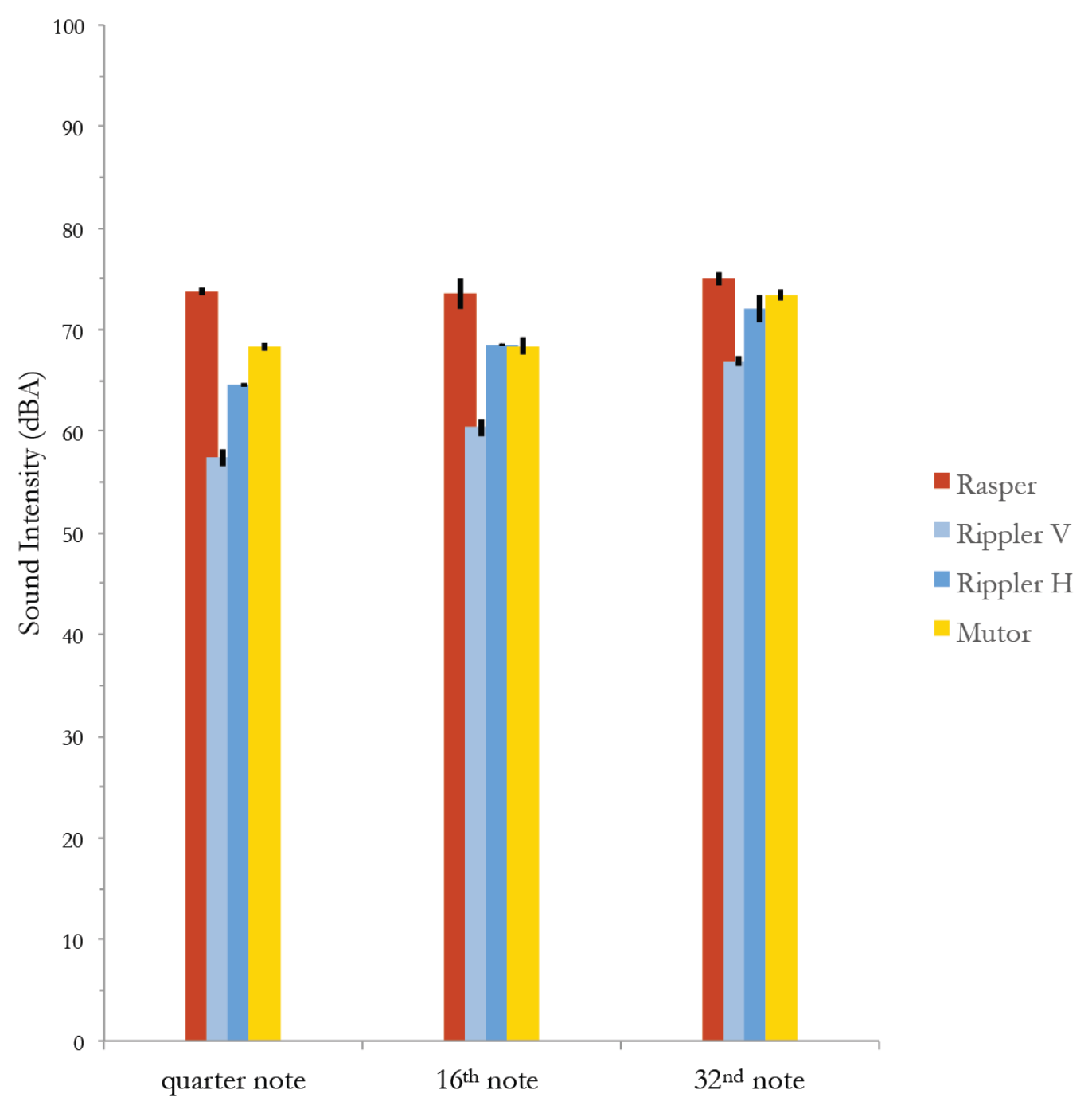

Figure 3-42. Relative loudness of the sound-sculptures. 
Based on these results, Rasper is generally the loudest instrument and the V-model of Rippler is the softest. This maximum loudness difference is quite discernable for a one single quarter-note input. However, as the rhythmic density increases, this maximum loudness gap becomes smaller, to a point that in a sequence of $32^{\text {nd }}$ notes Rasper, Rippler (H-model), and Mutor's peak difference lies in an interval of less than $5 \mathrm{dBA}$, which is smaller than the threshold of loudness difference detection perceived by the ear at that intensity [54].

\subsection{Summary}

This chapter introduced Rasper, Rippler, and Mutor: three new mechatronic soundsculptures that were custom-designed and developed in realisation of one of the main objectives of this research. In terms of sonic characteristics, all three instruments generate sounds that are considered noise - both acoustically and socioculturally. Aligned with the objectives of this research, their noise-production mechanism, which is fully visible and accessible, is equipped with the ability to create recurring, pulsating, and metric rhythms. Each instrument has a limited timbral variety that can be utilised by applying different input values. This frequency and timbre response in each instrument, targets a different area in the audible frequency spectrum, which together form a wide range from high $(11 \mathrm{kHz}-13 \mathrm{kHz})$ to mid-range $(4 \mathrm{kHz}-7 \mathrm{kHz})$ to low-mid $(700 \mathrm{~Hz}-2 \mathrm{kHz})$ regions. The sound-generation in all three instruments occurs in an entirely acoustic and mechanical way, and is accompanied by strong visual elements provided by the use of synchronous bursts of white light. Regardless of the different apparatus they incorporate, these sound-sculptures follow the same aesthetic principles, where machine-made noise is brought back to the foreground through employment of grid-based rhythmic structures and emphasis on the physical and visual aspect of the work. The next chapter discusses three original compositions in which these mechatronic sound-sculptures are used as tools to construct new modes of realisation with regard to some compositional paradigms. 
The work presented in this chapter resulted in the following publications:

Mo H. Zareei, Ajay Kapur, Dale A. Carnegie. "Rasper: a Mechatronic Noise-intoner," In Proceedings of the 2014 International Conference on New Interfaces for Musical Expression (NIME), London, UK (2014).

Mo H. Zareei, Dale A. Carnegie, Ajay Kapur, and Dugal McKinnon. "Rippler: a Mechatronic Sound-sculpture," Journal of Comparative Media Arts 1 (2015).

Mo H. Zareei, Dale A. Carnegie, Ajay Kapur, and Dugal McKinnon. "Mutor. Drone Chorus of Metrically Muted Motors," In Proceedings of the 2014 International Computer Music Conference-Sound and Music Computing (ICMC-SMC), Athens, Greece (2014). 


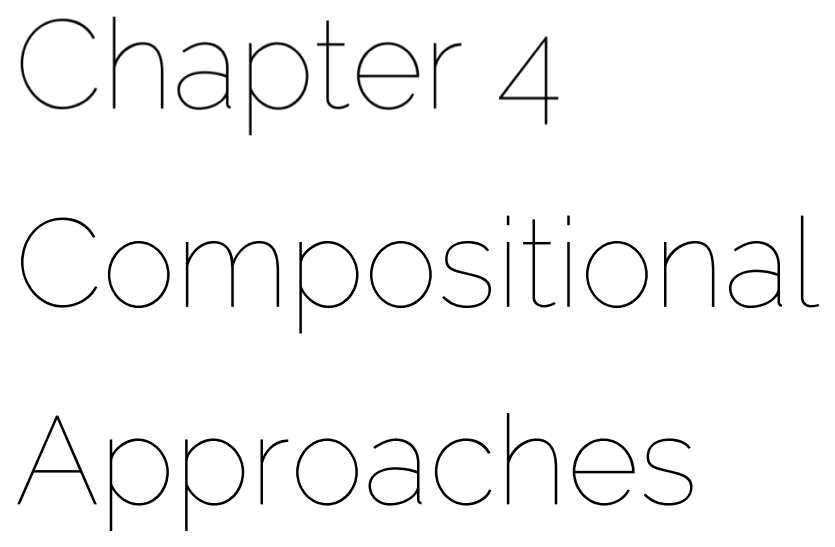

\subsection{Overview}

This chapter discusses three new compositions that incorporate the mechatronic soundsculptures developed in this research. Each composition belongs to an area of sound art that is in one way or another (ideologically, contextually, or aesthetically) connected to the ideas forming the body of this research. The areas targeted by these three compositions are minimalist music, algorithmic music, and urban soundscape music.

Each of the following sections discusses one of these works. Followed by an introduction on the particular area of sound art that the composition is connected to, each section provides a discussion on the links, influences, and motivations instigating the idea behind the work. Each section then describes the compositional strategies and techniques that are used in the creation and development of the piece, along with instances of the actual realisation of the work. 


\subsection{Remodelling Early Minimalist Composition}

\subsubsection{Introduction: Minimalist Music}

According to Edward Strickland, "in its simplest definition, Minimalism is a style distinguished by severity of means, clarity of form, and simplicity of structure and texture" [55]. The term was initially applied to a movement in visual arts in which the artist's tendency, as Kenneth Baker argues, was to "present as art things that are - or were when first exhibited - indistinguishable ... from raw materials or found objects, that is, minimally differentiated from mere non-art stuff' [56]. In the words of Keith Potter, Minimalist art "experimented with the limits of art by asking how many of the elements traditionally associated with it could be taken away to leave something which could still be considered art" [57]. Strickland argues that Minimalism is a form of art that "makes its statement with limited resources", is "prone to stasis", and "resistant to development" [55]. In this way, he states that in a musical context, Minimalism is manifested as repetitive modules, static harmonies, drones, and silences. In his article "What is Minimalism Really About?", Tom Johnson's immediate response to this question is: "it has a lot to do with repetition" [58]. Summarising Johnson's article, Potter cites "repetition", "tiny variations", "hyper clarity", and "making music less dramatic" as chief attributes of Minimal music [57]. Although repetition is a "basic structural components of all forms of classical music", as Strickland remarks, in Minimal music, it is implemented in an "overt and immediately audible" manner, and is the predominant structural principle [55]. According to Potter, "by selecting some of the oldest and most familiar building blocks of music, and subjecting them to the radical scrutiny afforded by remorseless repetition, [Minimalism] takes on the challenge of revitalising the most hackneyed and debased musical currency available" [57].

Minimalist music shares two major common features with the concept of "noise on the grid" undertaken by this research. On one hand, "its focus on sensations based on the direct perception of object forces [its audience] to a radical reconsideration of those objects". Where the object is a sound-object, this research's focus on the trivial noises of urban technological life and its effort to aestheticise them represents an analogous 
approach. Second, the rigorous structural incorporation of repetition in Minimal music can be interpreted as an equivalent of the emphasis on the grid-based rhythmic scaffold discussed in this research. With this in mind, as the first instance of incorporation of the mechatronic sound-sculptures in a compositional context, this section presents a new composition in appreciation of the works of one of the chief precursors of Minimal music, Steve Reich.

\subsubsection{Pulse Music of Steve Reich}

La Monte Young, Terry Riley, Philip Glass, and Steve Reich are widely known as the founding fathers of Minimal music. Regardless of the complexity and richness difference between their early and recent works, Potter points out that all four composers have been "continuing to activate their music with the crucial ingredient of repetition - or in Young's case, more often sustained sounds" [57]. According to Michael Nyman, "Riley's major achievement has been the installation of regular pulse into experimental music" [59]. Reliance on repetition is, however, even more significant in Reich's work. In fact, in an interview with Nyman, Reich stated that he preferred the term 'pulse music' to Minimal music [60]. As Potter notes "pulse-dominated percussion-based music had in fact fascinated him since he was a child" [57]. Repetition, according to Nyman, "is a 'local' device by which Reich realizes his concept of 'music as a gradual process"' [59].

In his article "Music as a Gradual Process", Reich expresses his intentions on creating a piece of music that is "literally" a process [61]. His early tape works such as It's Gonna Rain (1965) and Come Out (1966) that are entirely based on phasing effects, explicitly manifest his approach:

In the process of trying to line up two identical tape loops in some particular relationship, I discovered that the most interesting music of all was made by simply lining the loops up in unison, and letting them slowly shift out of phase with each other. As I listened to this gradual phase-shifting process I began to realize that it was an extraordinary form of musical structure [61].

As Potter explains, "Reich's discovery of phasing in fact has much in common with Cageian musical practice ... [in that is has] its roots in the observation of a process 
happening independently of its composer's conscious control" [57]. However, as Reich argues himself, Cage's processes were solely compositional and not audible, whereas for him, being able to "hear the process throughout the sounding music" is key [61].

Rhythmic repetition underscores all of Reich's works since It's Gonna Rain. Starting with Its Gonna Rain, the use of the gradual phase-shifting process continued "in every piece from 1965 through Drumming in 1971, with the exception of Four Organs" and was finally ended in late 1971 with Clapping Music [61]. Mentioning the pivotal role of handclapping in a typical African ensemble, Potter refers to this piece as one of the instances of the African music influence in Reich's work.

Clapping Music was written for two pairs of hands of two performers, one of which clapped a basic rhythmic pattern repeatedly, as the other shifted the downbeat to the succeeding beat after a number of repeats. On this piece Reich writes:

The basic difference between these sudden changes [in Clapping Music] and the gradual changes of phase in other pieces is that when phasing one can hear the same pattern moving away from itself with the down-beats of both parts separating further and further apart, while the sudden changes here create the sensation of a series of variations of two different patterns with their downbeats coinciding. In Clapping Music it can be difficult to hear that the second performer is in fact always playing the same original pattern as the first performer, though starting in different places" [61].

As Potter remarks, "even in a simple piece such as this, audibility of process is relegated in favour of its broader rhythmic consequences" [57]. The reliance on rhythmic repetition and the high degree of variety and complexity derived from a basic unit achieved in Clapping Music, in addition to its non-existing melodic material and minimal involvement of timbral change, made it an inspiring model for a new piece composed for the mechatronic sound-sculptures developed in this research.

\subsubsection{Rasping Music: an Audiovisual Composition}

Inspired by Steve Reich's Clapping Music, Rasping Music (Figure 4-1) is a composition based on phasing rhythms. Similar to Reich's work, the entire piece is a process in which various potentials of a simple rhythmic unit are explored and experienced by applying it 
to four Raspers, while shifting the downbeat of one at a time. The rhythmic competence of Rasper, in addition to its programmability, makes it capable of following any desirable rhythmic pattern perfectly and shifting the downbeat flawlessly whenever required. Nevertheless, despite the wide rhythmic capabilities of the instrument, the initial rhythmic units written for this piece are intentionally composed to be simple and basic 4/4 patterns. This is done in an attempt to comply with the Minimalist ideology influencing the composition, in which sophisticated results are achieved gradually through a process, that is rigorous exhaustion of the minimal compositional material employed by the composer.

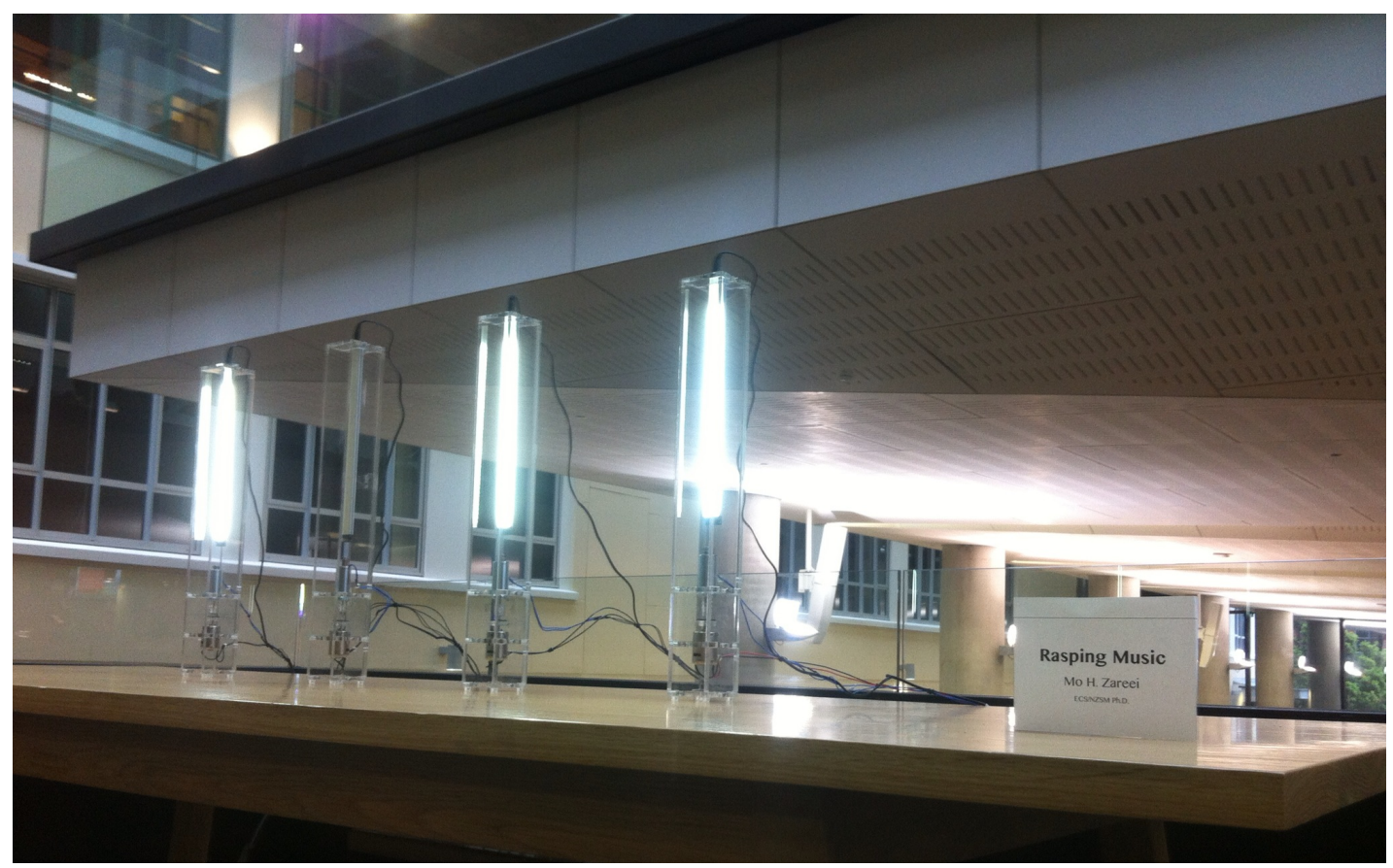

Figure 4-1. Rasping Music: installation realisation at Sonic Arts and Engineering Expo 2013, Victoria University of Wellington.

\subsubsection{Realisation}

The piece comprises three sections, each starting with a different rhythmic unit as the initial pattern. Four Raspers have been used in order to achieve a wider variety of interlocking patterns. During each section, all four Raspers start with the same rhythmic pattern and after every four bars of repetition, the downbeat of one of them is shifted forward, except for one, which keeps the original downbeat throughout each section. In 
each section, shifting downbeats and morphing of the interlocking patterns continue to the point that the downbeat of one of the three phasing Raspers is switched back to the original position. Then, when the Rasper keeping the original downbeat starts following the next rhythmic unit, the other three also move to the new section one at a time, every four measures. Figure 4-2 demonstrates the initial rhythmic unit of each section in order of appearance in the piece.

(a)
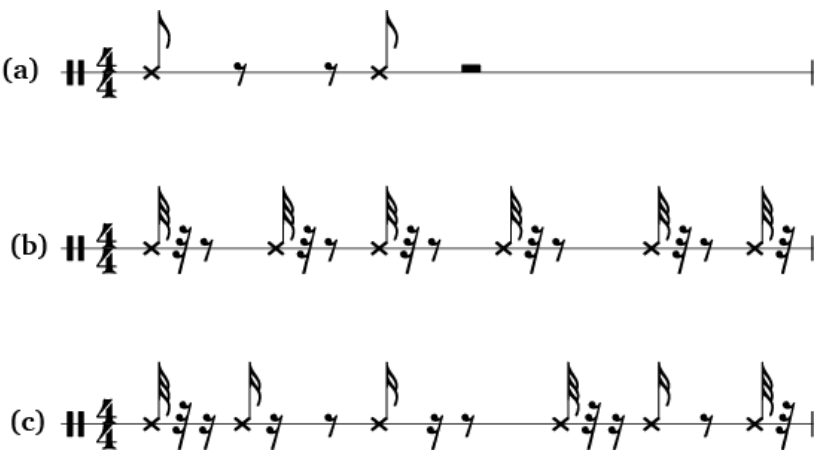

Figure 4-2. Rasping Music: rhythmic units.

As can be seen in this figure, the degree of rhythmic complexity increases with each succeeding section. The first section starts with the very simple pattern, in order to make it easier for the audience to notice the phasing process. After four measures of all instruments repeating this pattern, the phase-shifting starts according to the following criteria:

1. The downbeat of one Rasper is shifted forward by an $8^{\text {th }}$ note,

2. After four measures, the downbeat of the second Rasper is shifted by another $8^{\text {th }}$ note in relation to the last step (that is a quarter note from the original position),

3. After another four measures, the downbeat of the third Rasper is shifted by another $8^{\text {th }}$ note in relation to the last step (that is a dotted quarter note from the original position),

4. After another four measures, the downbeat of the first Rasper is shifted again by an $8^{\text {th }}$ note in relation to the last step.

As the fourth Rasper holds on to the original downbeat, this algorithm continues until one of the Raspers has cycled though the whole bar and reached the original downbeat 
again. In this way, a variety of different interlocking rhythms that are all derived from the original pattern are experienced as the piece moves into the second section (Figure 4-3).

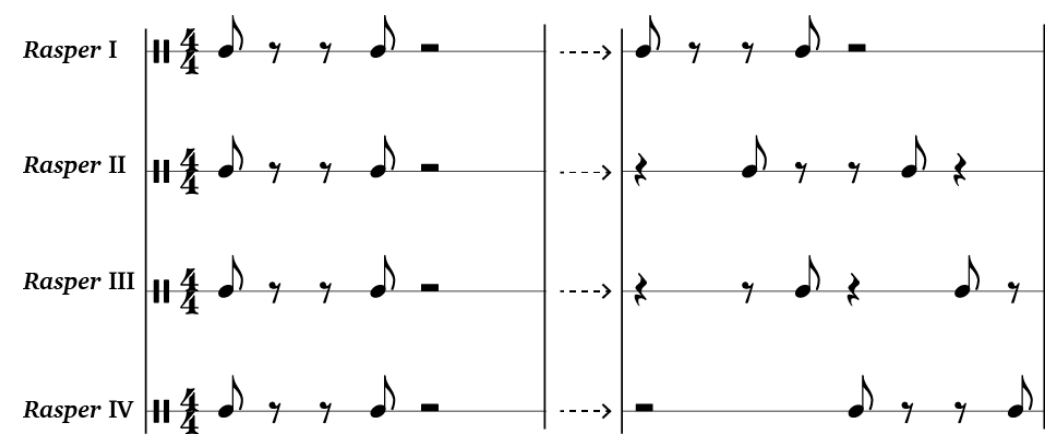

Figure 4-3. Rasping Music: development of section (a).

The development of section (b) is based on the exactly same criteria as the first section, and the only difference is the relatively more complex rhythmic unit. In section (c), however, the phasing is accomplished by shifting the downbeats by a $16^{\text {th }}$ note instead of an $8^{\text {th }}$ note. This opens up the chance for creating more interlocking and morphing patterns, and raises the rhythmic complexity of the work as the piece moves towards the end. By the end of section (c), all Raspers move back to the beginning of the section (a) one at a time, and once in sync, repeat the initial pattern for four measures and finally end on the same simple and synchronised rhythmic state in which the piece started.

The patterns are programmed in Ableton Live and in the form of MIDI clips, and phase-shifting is accomplished by adjusting the Start Markers. MIDI velocity values of 127 are sent to the solenoids to create the pulses. In order to narrow down the focus on the phasing process, motor speeds are kept constant by applying MIDI velocity values of 100 to the motors throughout the entire piece. Figure 4-4 provides an outline of the system overview.

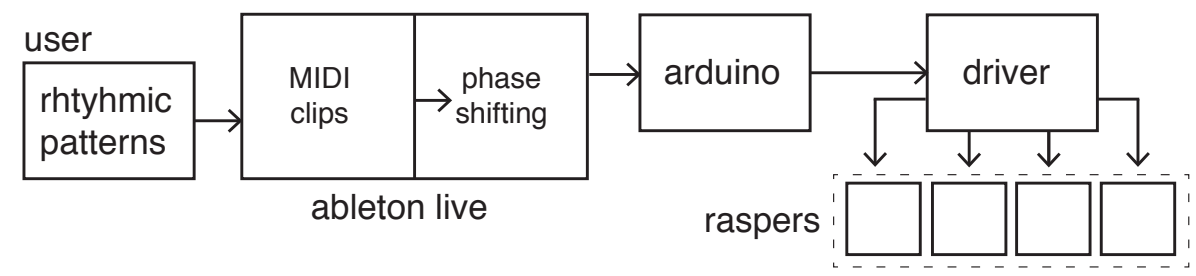

Figure 4-4. Rasping Music: system overview. 
Rasping Music has been realised both as an installation and a performance. In the installation setting, the piece cycles back when it reaches the end of the third section. As a performance the piece has been performed at Wellington City Gallery as part of the Sound Full festival in 2013, and the Adam Concert Room in the Composers Competition 2014, winning the $3^{\text {rd }}$ composition prize of the competition. As an installation, the piece was premiered at the Sonic Arts and Engineering Expo 2013 at Victoria University. It was also featured in Wellington Lux 2014 international festival as a ten-day long exhibition in Wellington city public space (see figure below). Rasping Music was the winner of $1^{\text {st }}$ Prize for Sound Art in the Sonic Arts Award ${ }^{19} 2015$, among a total of 71 submitted artworks.

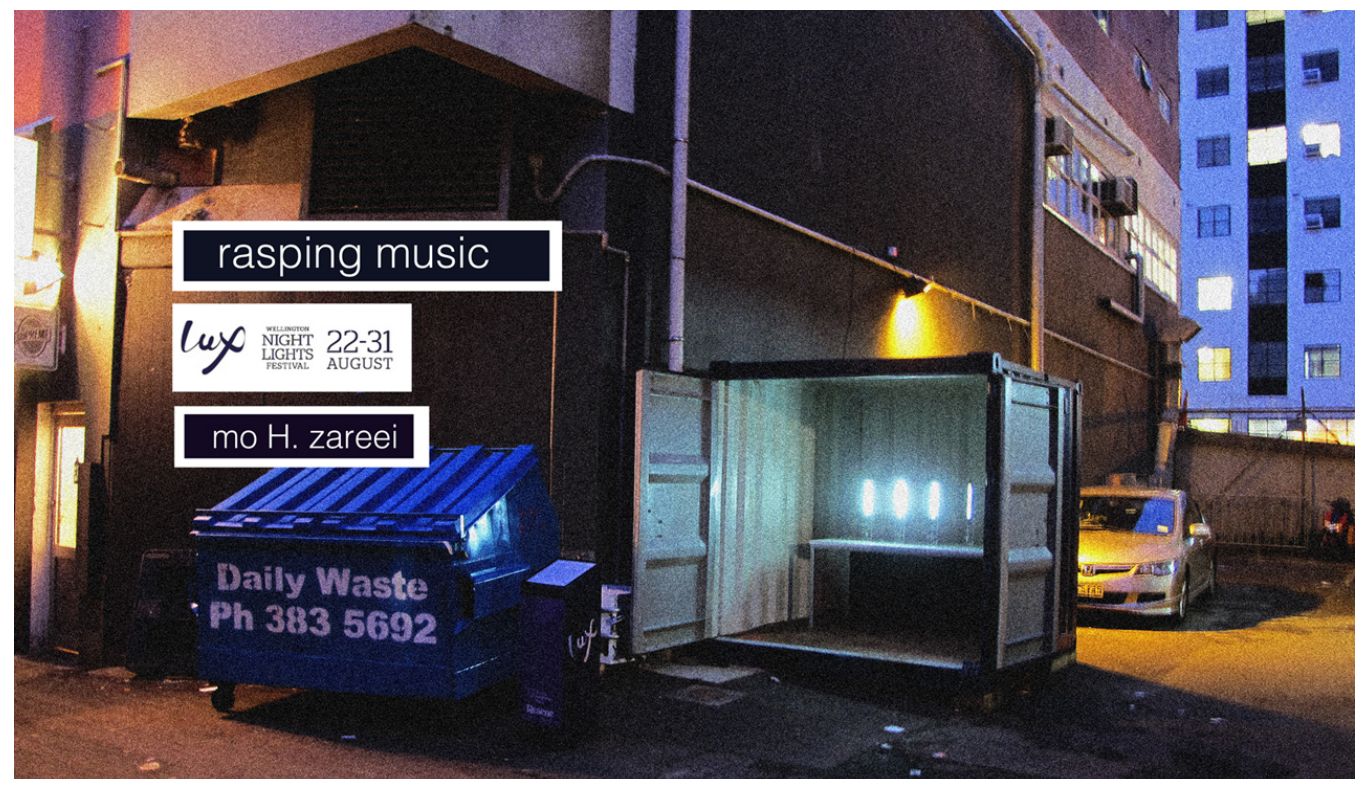

Figure 4-5. Rasping Music: audiovisual installation at Lux 2014, Wellington, New Zealand.

\subsubsection{Conclusion}

This section introduced Rasping Music: a minimalist audiovisual piece composed for the mechatronic sound-sculptures developed in this research that pays homage to one of the prominent early examples of Minimal music, that is Clapping Music. In doing so, the piece

${ }^{19}$ The Sonic Arts Award is a highly prestigious annual award selected by an international jury of artists and researches, aimed at the production and promotion of sonic arts at an international level. (www.sonicartsaward.it). 
remodels the key principle behind the rhythmic structure of Clapping Music, that is phasing downbeats, and uses it as the primary compositional approach in creating the rhythmic scaffold through which the noise of the mechatronic sound-sculptures is regulated. The next section introduces NOISE SQUARE, another audiovisual composition employing the mechatronic sound-sculptures that somehow incorporates a similar approach in construction of its minimalist compositional body. The rhythmic structure in NOISE SQUARE is, however, not based on a set of pre-composed patterns emerging from a process, but created through a set of generative algorithms.

\subsection{Physical Sonification of Cellular Automata}

\subsubsection{Introduction: Algorithmic Composition}

Quoting Christopher Strachey, Roger Alsop describes an algorithm as a "procedure for performing a complicated operation by carrying out a precisely determined sequence of simpler ones" [62]. When the complicated operation is the act of composing music, an algorithmic composition could be used, as Jacob argues, to produce something that is somehow different than what the composer would have composed on his/her own [63]. Dividing "creativity" into two different modes, one derived from inspiration and epiphany and the other, an offspring of hard work, Jacob states that an algorithmic composition system helps "reproduce the composer's creative methodology when the composer is in the 'hard work' mode" [63]. He regards algorithmic composition as a "compositional tool that simply makes the composer's work go faster" [63]. Alsop calls it a tool that "relieves the composer of many decisions" [62].

Algorithmic composition as the application of a set of well-defined rules to the process of composing music, as Jacob argues, is as old as music composition [63]. Nevertheless, on a more narrow view, Gerhard Nierhaus argues that algorithmic composition, that is, "composing by means of formalisable methods" is at least a century old. He mentions Arezzo's invention of automatic text-to-melody conversion as one of the early examples of algorithmic composition and refers to Schoenberg's twelve-tone 
technique, as well as to Bach's much earlier "The Art of Fugue", as well-known examples for development of formal complexity in generation of Western music [64].

The involvement of a generative process of some sort in a compositional system opens up the possibility to generate different outputs from the same inputs. Alan Dorin argues that "the computer with its limited set of instructions and operations, but with its huge capacity for the manipulation of representors ${ }^{20}$ provides a practical alternative for the artist interested in exploring generative processes" [65]. From another angle, Jacob regards the computer's incapability to defy well-defined sets of rules as a valid reason for its use in creative and process-based procedures when in "creativity-through-hard-work" mode [63].

As the first computer-assisted piece of algorithmic music, perhaps Hiller and Isaacson's Illiac Suite (1956) along with the works of Koenig and Xenakis should be viewed as the foundation of the following movement of algorithmic compositions [64]; a movement which can be expanded into a number of different sub-branches that are introduced in the next section.

\subsubsection{Classification}

In his book Algorithmic Composition, Nierhaus lays out the different classifications of algorithmic composition based on their methodology and mechanism, and expands each class in a different chapter. Based on his classification, algorithmic compositions can be categorised into the following classes:

1. Markov Models

2. Generative Grammars

3. Transition Networks

4. Chaos and Self-similarity

5. Cellular Automata

6. Neural Networks

7. Artificial Intelligence.

\footnotetext{
${ }^{20}$ In this Article, Dorin defines a representor as a 'thing' that represents.
} 
Nierhaus states that among these various methodologies, some - for example, Markov models - are primarily used in the context of 'style imitation' ${ }^{21}$ and some (such as cellular automata) are for 'genuine composition'22, while others - such as generative grammars - lie in a grey area in between the two [64]. As Jacob argues, whatever the chosen methodology is, the success of an algorithmic composition relies deeply on the compliance of the algorithm with the composer's creative process [63]. According to Nierhaus, "if the specific properties of the applied algorithms are not suitably used for structure generation, the motivation for selecting them is questionable" [64].

In the context of this research, where grid-based rhythms are of essential prominence, the grid-based essence of cellular automata gives them an edge over the other algorithmic and generative methods. Along with an overview of different instances of using cellular automata in music which predominantly belong to the digital realm, this section introduces NOISE SQUARE: an audiovisual generative composition in which the evolutionary patterns of a 1-dimensional cellular automaton are transferred to the physical realm through the mechatronic sound-sculptures developed over the course of this research.

\subsubsection{Cellular Automata Music}

Cellular Automata were originally introduced in the 1960s by Stanislaw Ulam and John von Neumann in the course of their studies on growth and reproduction processes [66]. They are a dynamic system in which time and space are discrete. Considering that music is a time-based system in which "a finite set of discrete values (such as musical notes, rhythms, etc.) evolve in space and time" [67], cellular automata are highly applicable to musical systems.

Cellular automata comprise one or multidimensional arrays of cells. Each cell can have a finite number of states (such as dead or alive), which are determined by simultaneous local transition rules. Transition rules are extracted from configurations of a neighbourhood of cells of specific length or range. Stephen Wolfram made a

21 That is when the algorithmic procedures are applied to model a style that is established in musicology.

22 That is when the algorithmic procedures are applied in the context of the creation of a new musical piece of art. 
significant contribution to the study of cellular automata by making a qualitative classification of them [68]. He divided them into four general classes that would lead to:

- Homogeneous states (class 1)

- Periodic and cyclic patterns (class 2)

- Chaotic and random behaviour (class 3)

- Complex form (class 4).

According to Dale Millen, in the context of a musical composition based on cellular automata, the composer can select or define each of the features above (that is the number of dimensions, cells, and states, the range, the type of transition rules, and the initial state of the system) [69]. The first instance of cellular automata used in music is perhaps Iannis Xenakis' composition Horos (1986). According to Varga, Xenakis was particularly interested in using the simplicity of cellular automata process to create complex and sophisticated results [70].

An overview of different approaches in applying cellular automata to the production of electronic music and sound art is given in Burraston and Edmonds' article in Digital Creativity journal, sound synthesis and MIDI sequencing techniques being the prominent methods. [71] LASy and Chaosynth are two noteworthy examples of using cellular automata in sound synthesis. The properties of these synthesisers are examined in detail by Miranda [67].

The utilisation of cellular automata in the MIDI domain is discussed by Burraston et al. [73]. There is an extensive body of work dedicated to the design and development of MIDI-based cellular automata systems. Beyls, Millen, and Miranda were some of the early pioneers in this field who contributed to the movement from slightly different angles. As one of the first composers to experiment with cellular automata, Beyls investigated one-dimensional and two-dimensional cellular automata to develop systems equipped with a MIDI mapping process for both non real-time and real-time compositions and performances [74][75][76]. Millen and Miranda investigated systems (CAM and CAMUS) that were primarily based on two-dimensional and threedimensional models of Conway's Game of Life, this itself being the best known example of two-dimensional cellular automata [77]. The characteristics and functionality of these 
systems, in addition to a number of other significant MIDI-based cellular automata compositional platforms are discussed and compared in detail by Burraston et al. [73].

In an instance of using cellular automata in an audiovisual installation, Bill Vorn utilised a two-dimensional cellular automaton inspired by Conway's Game of Life, in his work Evil/Live in 1997 [78]. An 8×8 grid of cells represented by light bulbs were attached onto an aluminium structure, and the light patterns were constantly changed according to the cellular automata activity governed by the computer. Each light bulb also corresponded to an audio event that was generated by a sampler. The audio patterns were triggered via MIDI messages and played back through the loudspeakers that were also attached to the aluminium structure. Vorn has expanded this work into Evil/Live 2 and Evil/Live 3 by increasing the number of matrices and cells and adding new levels of interaction.

Another example of using cellular automata in an installation work is Alan Dorin's Liquprism [79]. Liquiprism is an interactive piece, based on the idea of generating polyrhythmic patterns through six different two-dimensional cellular automata that formed facets of a cube. Dorin's goal was to highlight the conceptual conflict between the inherent regularity and determinism of cellular automata, and the resulting complexity and fluidity that can be obtained by their interaction. Here the sonification is accomplished through connecting Liquiprism to an analogue modelling synthesiser with the triggered sounds varying between simple squelchy tones and pure sine tones: "Each face of the cube is assigned a different MIDI channel and each of these is assigned a different voice on the multi-timbral synthesiser" [79].

In a rather rare instance of using cellular automata in an interactive live-performance involving musical robots, where the sonic output is a result of a physical process rather than a digital one, Jingyin He has applied the two-dimensional Game of Life cellular automata onto a grid-based MIDI controller in order to interact with a number of mechatronic idiophones and membranophones (The KarmetiK Machine Orchestra) [35][80].

In his 1990 article "Generative Processes and the Electronic Arts", Dorin mentions the wind-chime as a physical example for such generative process-based systems [65]. 
Yet, referring to the physical processes as "exceedingly complex" and difficult to control, Dorin argues that the computer "provides a practical alternative for the artist interested in exploring generative processes" [65]. That is perhaps one of the main reasons underlying the occurrence of the great majority of the instances of using cellular automata in music in the electronic sphere.

\subsubsection{NOISE SQUARE: an Audiovisual Composition}

With the rapid developments of DIY technologies in recent decades, it is now possible to achieve a well-defined and highly controlled sonic output through a mechanical process and in a physical manner. The combination of generative algorithms and mechatronic musical instruments in works of Arne Eigenfeldt are good examples of this in the musical robotics field [81].

Accordingly, the mechatronic sound-sculptures developed in this research can be used as a suitable medium for realising this idea in the field of mechatronic soundobjects. The programmability and autonomous capabilities of these instruments, in addition to their high degree of responsiveness and predictable functionality calls for their utilisation in the context of generative art. With this in mind, this section discusses NOISE SQUARE: an audiovisual work in which generative patterns of a 1-dimensional cellular automaton are sonified through a set of four mechatronic sound-sculptures.

Cellular automata in fact share the very structural essence of the temporal scaffold of the mechatronic instruments developed in this research, that is, employing basic binary elements to create a much more complex output. Among the three instrument-types, the minimalistic, cubic, and grid-based form of Mutor, along with the synchronous square light panels, sit perfectly with the black and white cells of the cellular automaton grids in terms of aesthetics. Considering the visual significance of an audiovisual installation work, this concrete and tangible connection between the instrument and the input source can further strengthen the audiovisual expressivity and tighten the semanticsyntactic relationship. 


\subsubsection{Realisation}

The piece employs a set of four Mutors, placed next to each other in a row. A 1dimensional cellular automaton of four cells with the neighbourhood range of one is used as the generative input source, each Mutor representing one of the cells. A grid of $4 \times 15$ cells - whose physical dimensions are mapped to the dimensions of the Mutors - is projected on a screen behind the row of Mutors. During the installation, this $4 \times 16$ grid displays the states of 16 sequential generations of the cellular automaton (see Figure 4-6).

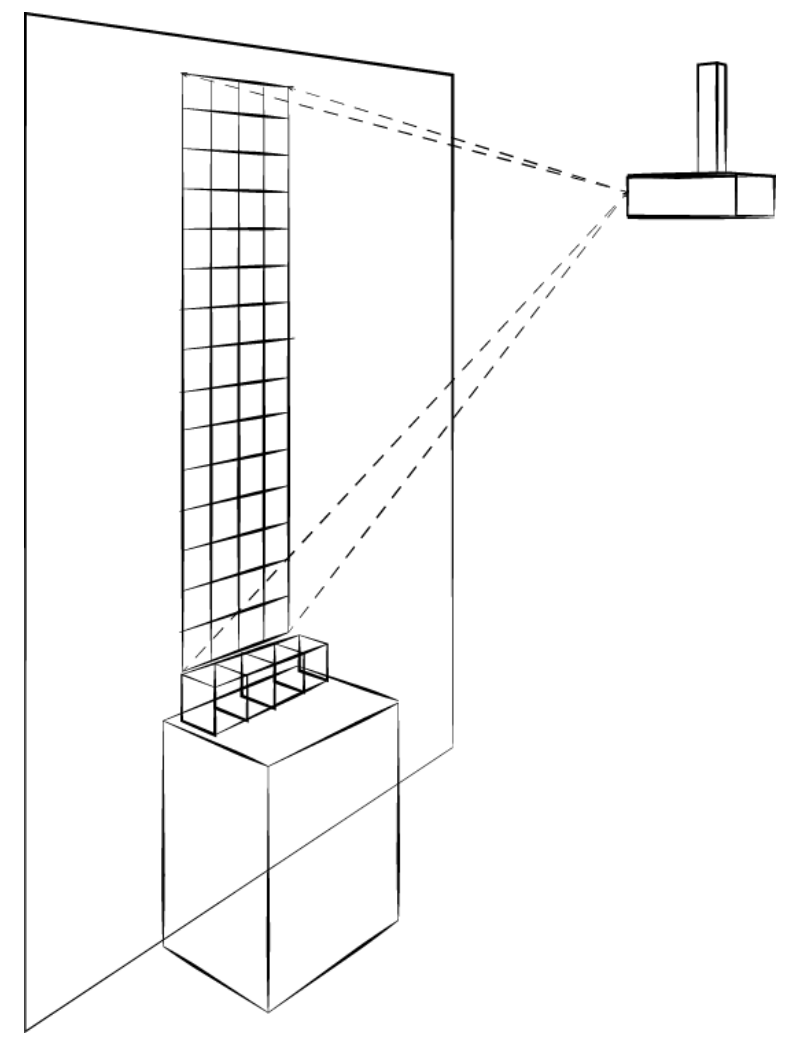

Figure 4-6. NOISE SQUARE: set-up.

The cellular automaton is generated in the programming language Processing and the cell-states are transformed into MIDI messages, which are then sent to Mutor's driver board using Ableton Live. Figure 4-7 demonstrates the system overview. 


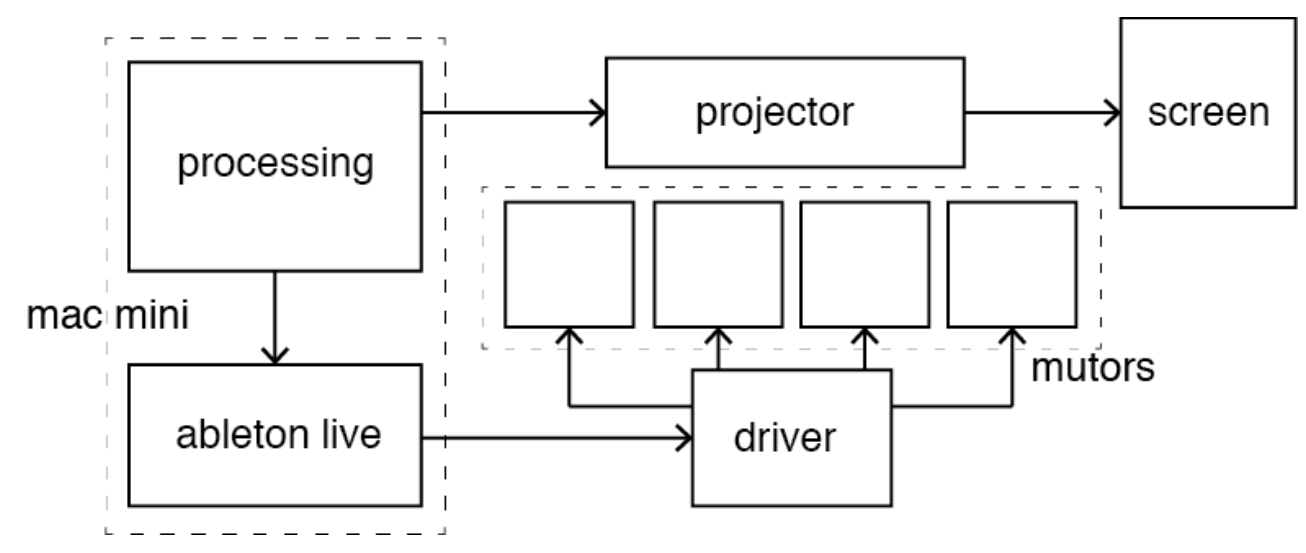

Figure 4-7. NOISE SQUARE: system overview.

As the cellular automaton develops, the generations (rows) continuously step through the grid from the bottom (Mutors) row to the top of the grid. The cell-states in the bottom row determine the status of the Mutors. Therefore, four columns of the grid can be thought of as four vertical step sequencers constantly driving the instruments.

Initially, a random rule-set (among the 256 possibilities) will be applied to the cellular automaton, and its corresponding number will be scaled down to the range of 0 and 127 for MIDI conversion. This scaled number is then used as the MIDI velocity input value for the motors, creating a rich and continuous drone chorus. Contrary to the standard practice of representing a live cell with black and a dead cell with white, the alive and dead cells are respectively represented by white and black squares. This justification is made in order to match Mutor's visual characteristic: a note-on input lights the LED panel up and puts the instrument in the unmuted state and vice versa. Therefore, in the case of this composition, a live (white) cell creates a MIDI message with the velocity of 127, which then lights the Mutor on and causes the solenoid to push out and open the cube's door. A dead (black) cell creates a MIDI velocity of 0 , turning the LED panel off, and returning the solenoid to rest position, which shuts the cube's door.

In order to create an accessible sense of rhythm, the cellular automaton is programmed to develop at the rate of 8 generations per second, giving each step the duration of a $16^{\text {th }}$ note in a BPM of 120 . Every 64 steps, that is 64 generations (or four measures) in 120BPM, the cellular automaton rules are programmed to reset to another randomly chosen rule, changing the tone of the motors and the evolutionary behaviour 
that determines Mutors' rhythmic patterns. This is done in order to experience different evolutionary patterns that result in different rhythmic behaviour. Additionally, resetting the rule number to a different number every 64 steps help avoid getting stuck by rulesets that belong to a homogeneous state that can lead to static behaviour, and potentially silence.

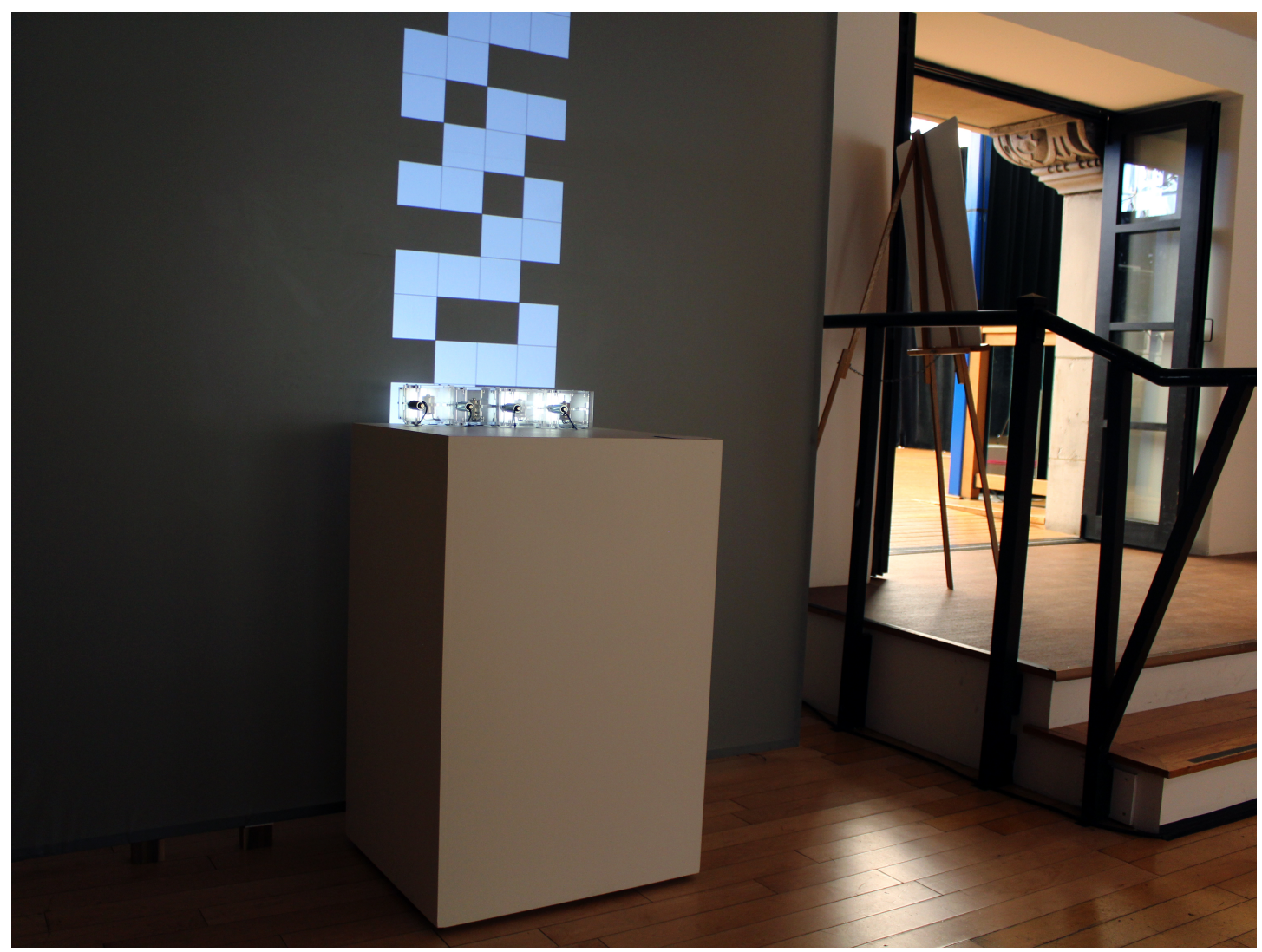

Figure 4-8. NOISE SQUARE: audiovisual installation at Vancouver Art Gallery, ISEA 2015.

NOISE SQUARE was exhibited in the $21^{\text {st }}$ Symposium on Electronic Art (ISEA2) at the Vancouver Art Gallery, Vancouver, Canada. The artistic programme of ISEA 2015 had an acceptance rate of only ten percent (from 1500 initial submissions).

\subsubsection{Conclusion}

This section discussed NOISE SQUARE: an audiovisual composition that employs generative patterns of cellular automata as an evolutionary rhythmic structure, and by sonifying them through the mechatronic sound-sculptures developed in this research, 
transfers them from the digital realm into the physical. The next section introduces an audiovisual piece entitled complex that also incorporates data sonification as the primary compositional approach. However, the data used in complex are not digitally generated using self-evolutionary algorithms, as they correspond to the recordings of an urban soundscape.

\subsection{Physical Re-sonification of Urban Noise}

\subsubsection{Introduction: Aesthetics of Urban Soundscape}

As discussed earlier in this thesis, along with the exponential rate of development in urban technological life since the Industrial Revolution, the sonic artefacts of the growing technologies have grown in quantity and variety. For more than a century now, city life involves immersion in a constant cacophony of machine noise. However, to what degree these sounds are attentively heard and noticed is the subject of debate. According to Fisher, city dwellers develop "the psychological capacity to ignore sound" [82]. This behaviour can be explained as a function of what Claire Taylor refers to as attention filter: "a selective machine" which "has the ability to tune out all the information that is superfluous to our concerns" and "is developed as a person matures" [12]. Indeed, "inattentive behavior toward environmental sound may be quite useful in urban settings", helping urbanites to "ignore sounds that would mask speech and hence information flow", or act as a defence mechanism when the urban "industrial sounds impinge on the edges of discomfort in frequency and intensity" [82]. After all, one common attribute of noise - at least from the sociocultural view - is its "unwantedness". To be able to ignore the unwanted can be a positive capability.

While it may be true that noises of the urban technological life are not made to create musical pleasure, does that necessarily mean that they cannot be pleasing to listen to? Criticising aesthetics theory's lack of attention to non-musical sounds of the environment, Fisher suggests that this negligence "in part reflects the fact that modern urban dwellers often ignore or suppress awareness of many of the sounds around them" 
[82]. Such sounds are unwanted aural artefacts of those technologies and urban inhabitants learn to ignore them, but as Fisher argues, "just because non-musical sounds are not intentionally produced to be pleasing to listen to, it does not follow that they are not pleasing to listen to" [82].

This section introduces complex: an audiovisual composition aimed at raising awareness and appreciation of the noises of modern city noise through creating a metaphorical representation of one of the most symbolic urban soundscapes, that is New York City soundscape, using the mechatronic instruments developed in this research. This is accomplished primarily through re-sonification and visualisation of the sonic data of New York City, collected from a big-data resource provided by the Citygram project $^{23}$. In the following sections, an overview of the concept of urban soundscape as an aesthetically valued source of sound is provided and the role of attentive listening in this process is assessed. Then, the compositional ideas and mapping strategies in the realisation of complex are discussed, following an introduction to the Citygram project.

\subsubsection{Urban Soundscape: From Attention to Appreciation}

Coining the term 'soundscape' as "an auditory analogue of landscape” [83], R. Murray Schafer was one of the key figures in raising awareness of the sonic environment [84]. His ideas, writings, and terminology continue to influence the field of acoustic ecology to this date. For Schafer, there is a significant difference between the natural sonic environment and the one created by human-made machines and urban technologies, to the extent that he regards to latter as lo-fi and the former as hi-fi. As Kelman explains, for Schafer "most of human history has been a sonic descent from the natural and harmonious into the mechanical and dissonant" [85]. According to Kelman, in expressing his preference for the natural soundscape over the noisy urban soundscape, Schafer claims that the original soundscape was quiet, but "doesn't explain what, where, or when this "original soundscape" existed" [85]. Therefore, while Schafer invites listeners to attend to the soundscape, he demands that they tune into certain sounds and

\footnotetext{
${ }^{23}$ http://citygram.smusic.nyu.edu
} 
ignore the rest [86]. In other words, his notion of soundscape "is lined with ideological and ecological messages about which sounds "matter" and which do not" [85].

A more comprehensive approach to the understanding of non-musical environmental sounds can be found in John Cage's idea of 'all-sound'. Like Schafer, Cage aims to foster attention to the soundscape, but unlike Schafer - as discussed in Chapter 1 - he does not make a rigid distinction between the natural and urban sounds:

Wherever we are, what we hear is mostly noise. When we ignore it, it disturbs us. When we listen to it, we find it fascinating. The sound of a truck at fifty miles per hour. Static between the stations. Rain. [3]

Cage's approach to the urban anthropogenic soundscape was in fact to draw attention to everyday non-musical sounds and noises, not least of all by integrating them in his music. Using a vast variety of non-musical and everyday objects in his concerts, he endeavoured to reveal the value - aesthetic and otherwise - of the noises of everyday life, and change the way his audience perceived such sounds. Writing about Cage's concerts, Douglas Kahn proclaims that "the noise in the city would not be physically diminished, but the city-dwelling concert-goers would accommodate themselves to it by appreciating it differently. [13]" The call for appreciation of the everyday sounds by Cage is aptly summarised by Alan Licht: "Cage is music world's answer to [Marcel] Duchamp" [44].

Preceding Cage's idea of 'all-sound', the celebration of urban noise by presenting it in a musical form is evident in works of Edgar Varèse and George Antheil. In The Soundscape of Modernity, Emily Thompson discusses ways that the noises of the modern city inspired Varèse and Antheil to create new kinds of music [87]. Remarking on the experiences of some of their (often well-known) audience, she describes how these concerts - although not always positively received - changed the way in which they experienced and perceived the urban noise:

By hearing Antheil's music, seemingly so much noise, when I actually came upon noise in reality, I found that I had gone up over it [88]. 
A thousand insignificant sensations have suddenly become interesting, full of character and meaning [89].

According to Thompson, "when this music was performed in places like Carnegie Hall, audiences were challenged to test their ideas about the distinction between music and noise. Some ... clearly developed a new way of listening, learning not only to celebrate the noise in music, but also to appreciate the music in noise" [87].

While Varèse and Antheil's works integrated sounds conventionally perceived as musical or non-musical so as to blur the distinction between the two, Futurist Luigi Russolo's Art of Noises was a perspicuous manifesto for the full appreciation of the industrial and urban noise, and also a clear rejection of traditional musical sound. As mentioned in 2.2.1, he believed that musical sound is too limited in its variety of timbres and wanted to "break out of this limited circle of sounds and conquer the infinite variety of noise-sounds" [2]:

We will amuse ourselves by orchestrating together in our imagination the din of rolling shop shutters, the varied hubbub of train stations, iron works, thread mills, printing presses, electrical plants, and subways [2].

As discussed previously, Russolo's Intonarumori were directly inspired by postIndustrial Revolution urban noise. However, unlike Cage who welcomed non-musical sounds of the everyday life in the very form and shape that they existed, Russolo's tactic was to "give pitches to these diverse noises, regulating them harmonically and rhythmically" [2].

In addition to their implicit methodical similarities in terms of noise generation, Rasper and Russolo's noise-intoners explicitly share this very conceptual ideology. Accordingly, the audiovisual composition described in the next section uses Rasper in creating a metaphorical (and conceptual) representation of the noises of the modern urban soundscape. 


\subsection{3 complex. an Audiovisual Composition}

Rasper receives MIDI messages as input and outputs rhythmically and timbrally regulated noise. Therefore, any stream of data that is eventually converted into MIDI messages would be able to drive the instrument. In a compositional approach where the output is particularly representative of noises of urban soundscapes, driving the instrument using some arbitrary input data seemed neither methodically convincing, nor conceptually strong. Therefore, in order to achieve conceptual cohesion, the Citygram project was chosen as the source of input data.

\subsubsection{Input Source: The Citygram Project}

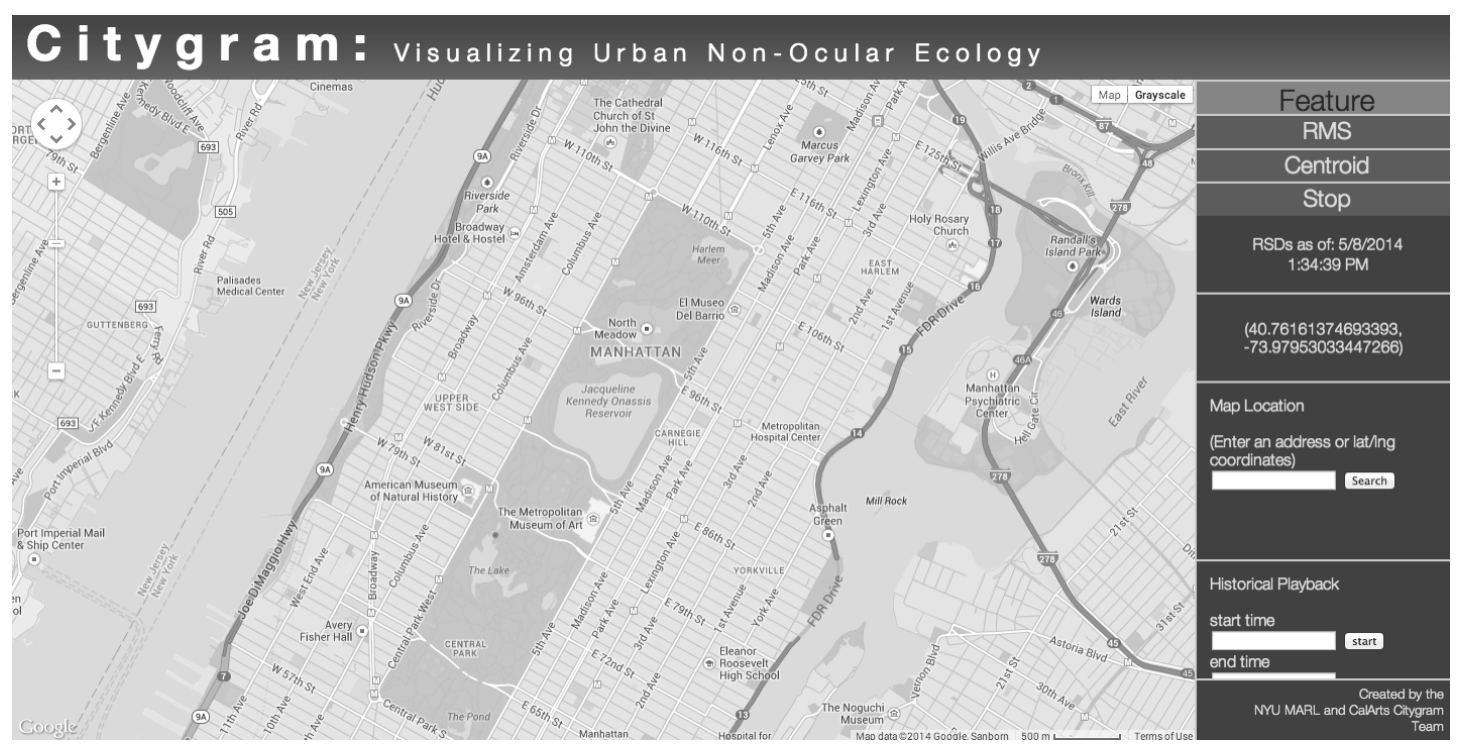

Figure 4-9. Screenshot from Citygram webpage.

Citygram is a project in which data from non-ocular energies of the urban environments are collected, visualised, mapped, and made accessible. In particular, the acoustic data of such environments is captured using remote sensing devices (RSD) and "the audio feature vectors are streamed to, or stored in, the Citygram database which can then be used for sonification and visualization" [90]. Currently, Citygram's focus is on the data from a limited number of small and manageable spaces, including a few blocks in Manhattan (Figure 4-9), the heart of one of the noisiest - and perhaps most symbolic urban soundscapes. 
Enabling strong conceptual cohesion was the main motivation in choosing the Citygram database in a composition focusing on aesthetics of noisy urban soundscapes. In fact, Rasper and Citygram can be understood as sharing a perspective on sound and the soundscape. First, they focus on noises of the urban soundscape and draw attention to aural phenomena that are normally ignored by the ears of city dwellers: the ubiquitous buzzing of the urban machines, or the incessant clamour of New York City. Furthermore, they employ strong visual accompaniments, so as to create unified audiovisual paradigms in which the worldly existence of noise is highlighted, in a way that is both accessible and engaging. Accordingly, complex, as a compound of both, reasserts the audiovisual noise of the urban life, underscoring its existence and affirming the need for awareness of such noise.

\subsubsection{Realisation}
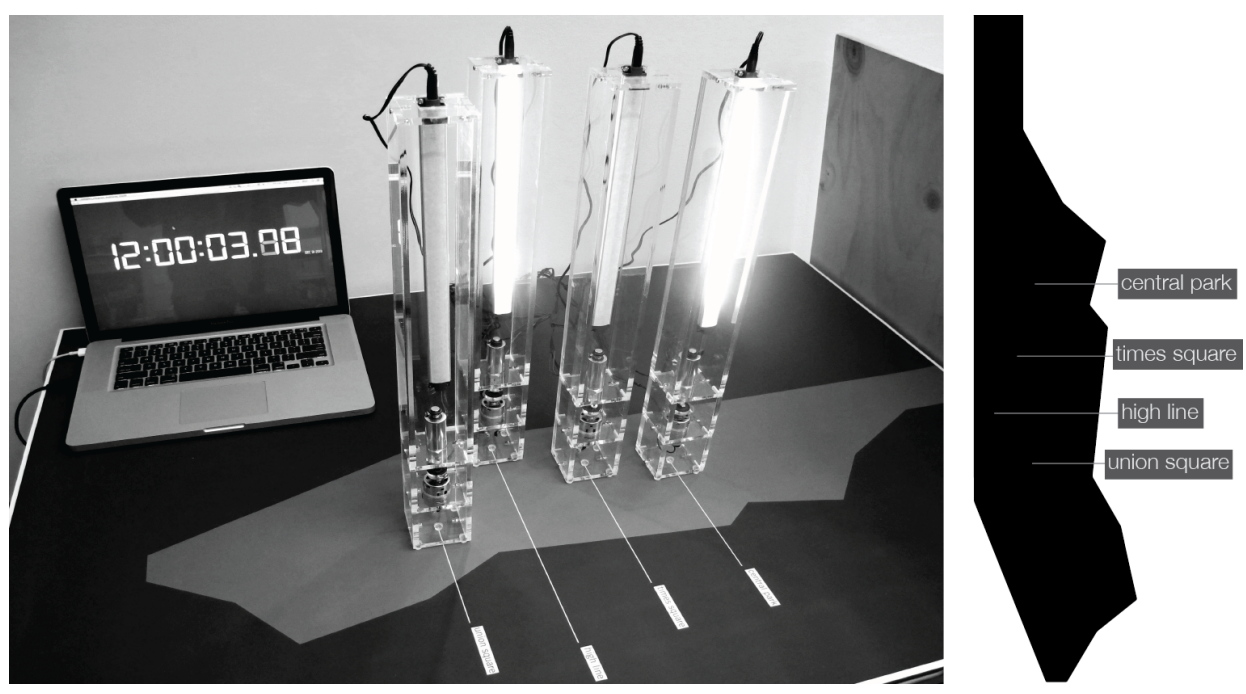

Figure 4-10. complex: demo realisation.

complex employs a set of four Raspers, playing interlocking patterns that are fed from the Citygram database. Four highly symbolic locations in New York City (Union Square, High Line Park, Times Square, and Central Park) were chosen as the input source for each Rasper. Therefore, the relative noise level of each neighbourhood is represented through the sonic output of the corresponding Rasper. In order to further strengthen the symbolic relationship of the instruments and their input source, during the installation they are 
placed on an allegorically designed map of Manhattan with the four neighbourhoods marked (Figure 4-10). This way, the intended symbolic connection existing in the aural domain has been extended to the visual realm as well: the sound-sculptures being reminiscent of Manhattan's skyscrapers, with their flashing lights recalling the densely lit up downtown and Times Square.

During the installation, data from these four locations are mapped onto the instruments according to the following criteria: RMS values extracted from the audio feature vectors are scaled from 0 to 127 , converted to MIDI velocity values, and if higher than a certain threshold, trigger the corresponding instrument. In other words, in the sequence of streaming data, only MIDI values higher than the threshold would be able to open a virtual gate and create a pulse, and those lower than the threshold would be filtered out. This is done in order to create a more dynamic rhythmic feel and avoid a continuous hum of noise. Calling the sequential input data and outputting the MIDI values are delayed by 100 milliseconds in every cycle, in order to reach a more accessible sense of rhythm, and to avoid a continuous stream of output events. The sequence restarts after cycling through 44,444 entities of all datasets synchronously. During each cycle, the entities' timestamp is screened on a monitor after being converted to the standard date and time. The data is collected from the Citygram dataset in the form of comma-separated vectors and the conversion to MIDI and filtering are done in Processing. The MIDI messages are sent to the micro-controller via Ableton Live, converted to a voltage using the driver board, and then used to run the instruments (see Figure 4-11).

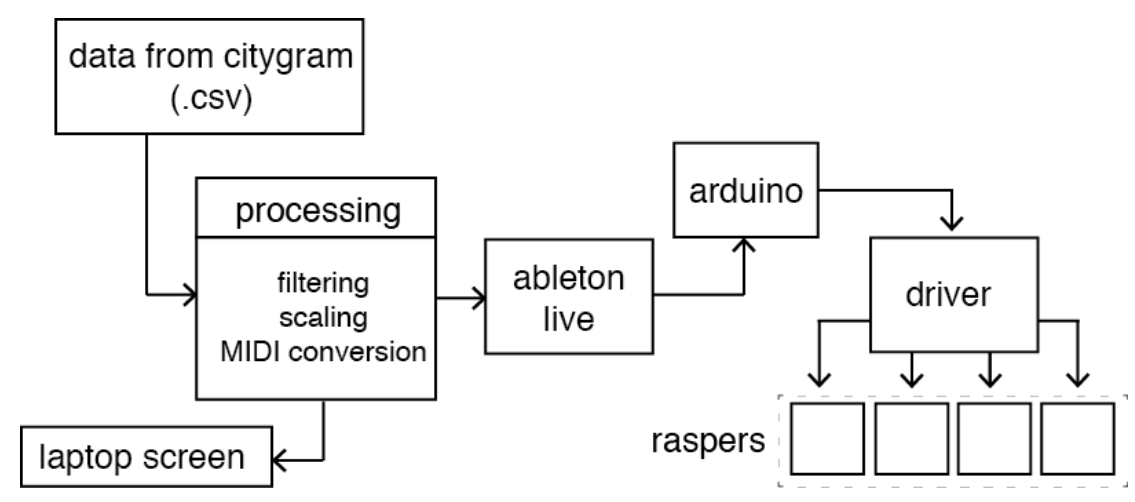

Figure 4-11. complex: system overview. 
The piece was exhibited at the International Conference on Auditory Display (ICAD) 2014 in New York, as one of the only four installation pieces selected to the artistic programme of the conference (Figure 4-12).

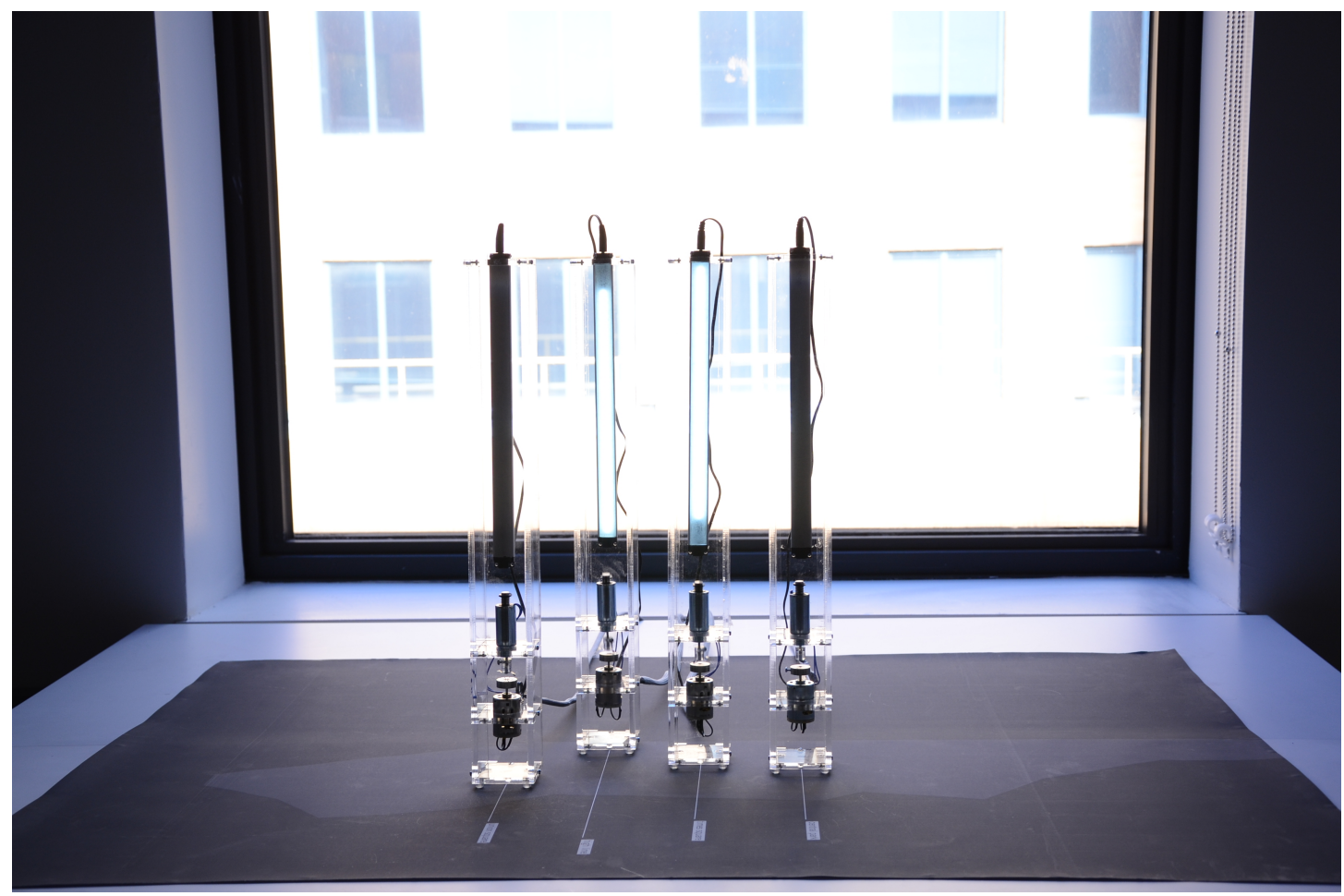

Figure 4-12. complex: exhibition at NYU magnet, ICAD 2014, Brooklyn, NY.

\subsubsection{Conclusion}

This section introduced complex: an audiovisual composition that undertakes physical resonification of noises of urban soundscape. In doing so, the piece creates a metaphorical model, in which four mechatronic sound-sculptures represent the noise levels of four symbolic locations in one of the noisiest urban environments today. In this way, the background noise of this urban soundscape, which is symbolised by the mechanical noise of the sound-sculptures, is aesthetically recycled and brought back to the foreground in an audiovisual manner. 


\subsection{Summary}

This chapter presented three new audiovisual works that integrated mechatronic sound art into three different compositional fields and practices. These compositional platforms were chosen based on the aesthetic, ideological, and technical links that they share with the sound-sculptures and the ideas behind their development. Using mechatronic sound-sculpture as a new medium, these works contribute to the selected compositional practices both artistically and scholarly. These contributions are reflected in the resulting awards, peer-reviewed publications, and international exhibitions.

The work presented in this chapter resulted in the following publications:

Mo H. Zareei, Ajay Kapur, Dale A. Carnegie. "Rasping Music: Remodelling Early Minimalist Music through Mechatronic Sound-sculpture," In Proceedings of the 2015 International Computer Music Conference (ICMC), Denton, TX (2015).

Mo H. Zareei, Dale A. Carnegie, Ajay Kapur. "NOISE SQUARE: Physical Sonification of Cellular Automata through Mechatronic Sound-sculpture," In Proceedings of the 2015 International Symposium on Electronic Art (ISEA), Vancouver, Canada (2015).

Mo H. Zareei, Dugal McKinnon, Ajay Kapur, and Dale A. Carnegie. “complex: Physical Resonification of Urban Noise," In Proceedings of the 2014 International Conference on Auditory Display (ICAD), New York, NY (2014). 
105 


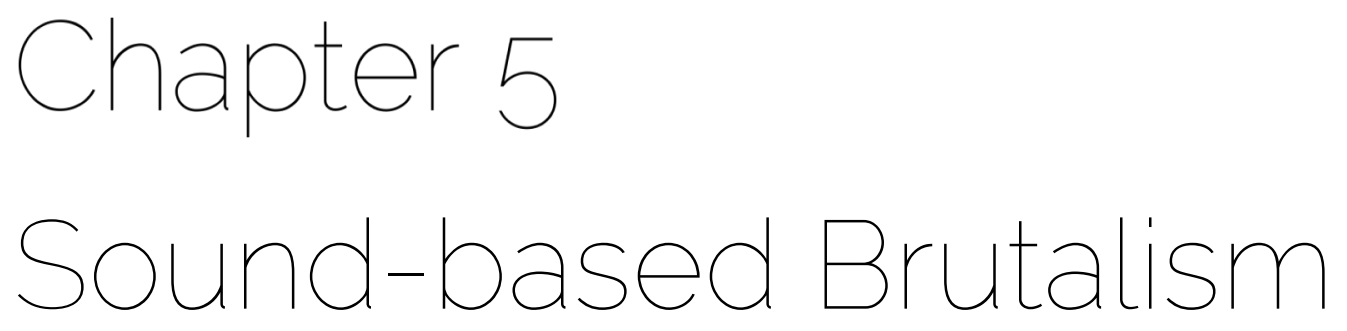

\subsection{Introduction: an Emergent Aesthetic}

\subsubsection{Overview}

With the idea of "channelisation of noise" as its main initiative, this research was developed in an effort to explore the potential aesthetic values of noise (in particular, non-musical mechanically generated sounds) through rhythmic regulation. To give accessibility to sounds that are conventionally perceived as musically inaccessible, the use of a temporal grid of metric rhythms, and the extension of modes of expressivity from the aural into the audiovisual realm were chosen as the main strategies. Employing mechatronics as an appropriate tool to merge these strategies, a number of soundsculptures were designed, developed, evaluated, and implemented in different compositional systems. Similar to the cross-medium series of works discussed in Chapter 2, these compositional methods belong to different areas of practice, technique, medium, and have different modes of realisation. However, there is an interlinking set of aesthetic principles that, regardless of these various technicalities, creates a coherent aesthetic context in which not only the discussed body of works, but also the developed soundsculptures and the compositions in which they were utilised can be situated. This chapter introduces this idea as an observation that arose during the development of this research, and as the outcome of reflection on the different constituents of the structure it is carried through. Presenting the concept and its practical outputs, this chapter aims to 
establish the necessary comprehensive aesthetic framework that, as argued in the following sections, is lacking from the academic literature of the recent years.

\subsubsection{A Need for Further Discussion}

One and a half decades after Kim Cascone articulated his concerns regarding the lack of exchange between the academic and independent sides of electronic and computer music [19], this issue does not seem to have been resolved. As argued in Chapter 2, the recurring presence of "beat" in the work of independent practitioners and its implicit association with popular music cultures are cited by both Cascone and Ben Neill as prominent reasons for this gap [19][28]. Around the same time, Joel Chadabe predicted the emergence of a "new cultural landscape, [one] not based on aristocratic and popular traditions" [29]. However, more than a decade later, the dismissive attitudes towards the independent approach that was "yet to be taken seriously by research-based computer music institutions" are still in place [91]. As Tony Myatt points out, "most research and academic writing about electronic and electroacoustic music is focused on music produced within academic communities" [92], leaving the body of work from independent artists and musicians largely unscrutinised, at least in terms of their musical and aesthetic features. The impact of this is seen, for example, in the rather equivocal terminology and classifications for works and genres: glitch, microsound, noise music, electronica, post-digital, and even sonic art and sound art, are overlapping terms often used interchangeably in writings about independent sound-based artworks. ${ }^{24}$ Such lack of consistency is perhaps partly due to the fact that even the limited amount of academic attention paid to these works, as Landy points out, is primarily targeted at their technical and technological characteristics, rather than aesthetic and theoretical ones [18].

Shortly after Landy made this remark, Tony Myatt, in his editorial note to the Organised Sound issue titled New Aesthetics and Practice in Experimental Electronic Music, called

\footnotetext{
${ }^{24}$ Landy uses the term sound-based music, as a slightly refined equivalent for sonic art, in reference to "the art form in which the sound, that is, not the musical note, is its basic unit", and suggests sound-based artworks for the non-Cageian reader, arguing that from a liberal viewpoint, sound-based artworks would be a subset of music [18]. Accordingly, 'sound-based artwork' is used here as an overarching term for reference to cross-medium artworks (such as electronic music, installations, sound-sculptures, etc.) in which the primary focus is sound.
} 
for a discussion of contemporary independent electronic music that "can provide accurate and factual descriptions of sounds and music in the context of contemporary cultures, economies and philosophies, particularly in relation to the function of music and of musical research within the societies and communities who support it" [92]. In parallel to this, he and three fellow researchers at the University of York instigated the New Aesthetics in Computer Music project in 2008, in an effort to explore the aesthetic principles evident in the works of a number of prominent independent electronic musicians, most of whom are yet to receive significant attention from scholars [93].

New Aesthetics in Computer Music features a series of interviews with these artists, among whom is Olaf Bender (aka Byetone), a co-founder of Raster-Noton, one of the most influential and aesthetically coherent record labels of the $21^{\text {st }}$ century's experimental electronic music scene. Regardless of their substantial contribution to the field in the past decade or so, works of just a few Raster-Noton artists receive only occasional mention in a limited number of academic writings. Excluding a few instances such as Adam Collis' article in the New Aesthetics issue [20], these mentions usually do not move beyond broad survey discussions as part of a more general discourse on glitch, microsound, noise, and sound art. For instance, Hegarty, Voegelin, and Demers briefly touch on the contributions of Carsten Nicolai (aka Alva Noto, the other co-founder of Raster-Noton), Ryoji Ikeda, and Pan Sonic to microsound and glitch [22][6][24]. Caleb Kelly's rather comprehensive encyclopaedia of glitch completely (and surprisingly) overlooks these artists, to the extent that Nicolai - who according to Demers "rivals Kim Cascone for the position of the world's most prominent microsound musician" [24] - is cited only once and amongst several other names, in the introduction of Kelly's book [21].

Even when discussed, works of these artists are often referred to individually (sometimes even in separate chapters and subsections) with a more comprehensive investigation of their aesthetic interconnections remaining to be undertaken. In this respect, Torben Sangild's analysis - despite its brevity - stands out, as he groups Ryoji Ikeda, Alva Noto, and Pan Sonic together in a genre he refers to as "minimal click", 
suggesting sonic dryness, repetition, use of sonic technological by-products, and lack of melodic material as some of their common aesthetic features [25].

It is the aim of this chapter to expand and build upon this feature of Sangild's writing, in finding a common ground between various strands of contemporary soundbased artworks, strands which have been overlooked in the academic literature of recent years. In doing so, this chapter ignores the specific technical and technological discussions that have led to the rather ambiguous terminologies outlined above, and instead suggests a cross-genre and multidisciplinary investigation that is concerned with neither the medium nor the technology, but the interrelating aesthetic principles of these works.

\subsection{Defining the New Aesthetic}

From the final years of the $20^{\text {th }}$ century onwards, there has been an emergent aesthetic phenomenon evident across a growing number of independent sound-based artworks, regardless of their genre, cultural context, or medium. This includes works by the cofounders of Raster-Noton (Nicolai, Bender, and Frank Bretschneider) and several artists on their roster such as Ryoji Ikeda, Aoki Takamasa, Mika Vainio (one of the Pan Sonic duo), audiovisual projects by Martin Messier, Nicolas Bernier, Ryoichi Kurokawa, and large-scale kinetic sculptures by Zimoun and Pe Lang. Ranging across laptop-produced compositions, audiovisual performances, installations, and sound-sculptures, these works share certain characteristics. Although approached very differently in terms of tools, techniques and media, they are connected through common aesthetic elements: avoidance of melodic and harmonic material to a complete or substantial degree through the use of sounds that are conventionally perceived as extra-musical, harsh, mundane, and unwanted (digital or mechanical noises, extremely high and low frequencies, or sonic glitches, by-products, and artefacts), along with extensive use of iterative sonic and visual materials presented in clearly defined and rigidly gridded structures, comprising highly repetitive macro or micro building blocks (multiple iterations of an identical sound 
source, sound-object, or image, as well as pulse-based rhythms, static timbres, repeated patterns, and recurring images and stroboscopic visuals).

Many of these elements are reminiscent of the "minimalist skeletalism", which Sherburne identifies in early Chicago house and Detroit Techno as a means to rid the music of everything inessential to the pulse requisite for the dance floor [94]. According to Sherburne, the same minimalist skeletalism is used by glitch and microsound "to approximate the form of dance music by substitution and implication, swapping out traditional drum samples for equivalent sounds sourced from pared-down white noise: click, glitch, and crackle" [94].

\subsubsection{Inaptitude of "Minimalist"}

It might be convenient to paint the works mentioned above with the same broad minimalist brush, given that there are certain attributes supporting this assumption: repetition and the reduction of the sound material to basic elements (sinetones, burst of white noise, clicks, or basic sound-generating objects) being the pivotal ones. For example, in his Mille Plateaux manifesto, Achim Szepanski refers to "click", one of the signature sounds of microsound and glitch, as "the introduction to the minimalism of the twenty-first century" [95]. The association with minimalism is also made by Taylor Deupree, the founder of $12 \mathrm{k}$ (another influential contemporary record label for independent electronic and computer music), as Sherburne quotes him saying that the central aesthetic of $12 \mathrm{k}$ is minimalism [26]. A minimalist aesthetic is also used by RasterNoton co-founder Carsten Nicolai, as the foundational concept underlying the label's aesthetic, which he described as an attempt to "release the music without anything except the music and what is necessary to carry it out" [96]. However, as the other Raster-Noton co-founder, Olaf Bender, states in interview with Myatt, while reduction of structure to its essential elements makes their music "in a way minimal", they do not actually follow the traditions of Minimalist music [97]. Sonya Hofer argues that "while [microsound is] described as "minimalist" by many, this minimalism [...] did not 
necessarily adhere to the formal tenets of Minimalism, capital M" [98]. ${ }^{25}$ This can be extended to the mechanically produced audiovisual works and sound installations mentioned above. For instance, Zimoun explains in an interview that his interest in simplicity, repetitive and reductive principles, and raw material gives "some kind of minimal aesthetic" to his work [100]. Similarly, as cited in Chapter 2, Nicolas Bernier's Frequencies (a) (2012), is described as a "not-quite-minimal" sound and light composition on his website [47].

In his “Thankless Attempts at a Definition of Minimalism” [101], Kyle Gann lists a series of features shared by early Minimalist music, a number of which are also widely used in the works discussed here (such as repetition, steady pulse, linear transformation, clarity of structure). Yet the artists mentioned above are hesitant in closely identifying their works with Minimalism, which can be perhaps explained by the historical gap between the two, the very different cultural contexts in which they operate, and the differences in their musical materials especially in terms of pitch use. In discussing the works released on Mille Plateaux on the Clicks + Cuts (2000) compilation, William Ashline writes that "the 'minimalism' invoked here is hardly adequate for defining the distinctive character of a rapidly evolving genre of electronic music", and is not "comparable to the experiments of Steve Reich and others in the milieu of the 1960s and 1970s avant-classical tradition" [102]. Here then, minimalism is used with the lower case m.

In an interview with Ashline, Ian Andrews argues that the minimalism of glitch and microsound is a "return to purity" of modernism and a "rejection of postmodernism", a phenomenon he claims lacks theoretical investigation [102]. Expanding the discussion to the cross-medium works mentioned earlier, the line taken in this thesis is that although these works utilise structural or stylistic audiovisual features that are aesthetically minimal (again: repetition, reduction, formal clarity, etc.), solely identifying them with the term "minimalist" does not suffice in terms of the aesthetic assessment that is the purpose of this chapter. To make this point clear, there exist a large number of sound-based

\footnotetext{
${ }^{25}$ Minimalism, used with the capital $\mathrm{M}$ and not the lowercase $\mathrm{m}$, "is restricted to those artists who shared a philosophical commitment to the abstract, anti-compositional, material object in the 1960's” [99].
} 
artworks that although sharing minimal aesthetics, do not necessarily belong to the focus group of this thesis. This includes numerous works of Taylor Deupree, William Basinski, Scott Morgan (aka Loscil), or Trimpin, whose works are respectively associated with microsound, ambient, ambient electronica, and kinetic sound-sculpture. The noticeable use of melodic phrases, harmonic layers, or conventional musical instruments, in addition to developmental and open forms in these works, even if minimal features are incorporated through use of repetition or limited set of materials, excludes them from the more focused aesthetic group that this research is concerned with.

\subsubsection{Towards a More Coherent Definition}

What unifies many works of those artists under discussion here is something more than their minimalistic approach to form and materials. It is a recapitulation and recycling of key modernist tendencies - not limited to minimalism - that is presented in an uncompromising, or even confrontational manner. Beyond the aesthetic of reduction, there is a form of sensory provocation, conveyed through insistent use of contemporary technological noise that links them historically to Futurism. Noise is key here, whether acoustic (digitally or mechanically produced irregular vibrations) or sociocultural (nonmusical sounds or sound-generating objects, high frequencies, or extreme dynamics). Whatever the source of noise, it is presented in a highly ordered and organised manner: either temporally (the binary pulsating beats of Pan Sonic's Kesto (234.48:4) (2004), or Alva Noto's unitxt (2008)), spatially (Zimoun's painstakingly arranged prepared motors, or Kurokawa's perfectly arched HD displays sequencing through video snippets of waterfalls in Octfalls (2011)), or both (the perfect geometry of monochromic lines, rectangles, and grids in Ikeda's visuals accompanying his pulsating noises in The Transfinite (2011), or Manabe's accurately arrayed spinning metal sheets producing techno-like patterns in Motor Music Test 55 (2013)).

The lack or extreme limitation of pitch material, discreteness of timbre, and the simple pulse-based and clear-cut on/off envelopes in these works, aligns them tightly with Wishart's concept of 'the lattice' on which conventional musical structure is constructed [5]. Here, however, the sonic material never develops across the underlying 
lattice, as the works are not organised into developmental or teleological forms. This is the case not only in installation pieces, but also in fixed-media audio works, for in both there are no dramatic build-ups, climaxes, or significant dynamic shifts. One can enter the gallery, or click the play button at any point in a piece, and the audible structure reveals itself within the next few pulses, beats, or bars. Due to this block-like temporality, the discourse of these works is largely defined by the grid itself, which unlike Wishart's lattice, is not used as a conceptual framework to build sonic architecture upon, but is emphasised as a dominant aspect of the work. Additionally, and regardless of the mode and medium of expression, a visual grid often accompanies the sonic one: tightly concurrent arrays of moving objects, stroboscopic bursts of light, and highly synchronous visuals constructed from basic abstract geometries are ubiquitous. Both sonically and visually, what make these works aesthetically consistent and enthralling is their direct and radically reductionist use of material, and the structural clarity of their grid-based forms: fluorescent lights, monochromic pixels, and basic noise-producing (physical or digital) objects that are meticulously formalised through iterated (temporally, spatially, or both) grids made of identical or tightly similar units.

These works may be highly elaborate in their patterning of a reduced material and formal palette, but they also avoid over-complexity. In Nicolai's Prototypes (2001) for example, "while the sound world is extreme in its register and exaggerated thinness of texture, the familiar language of the beat is never far from the surface, playing a critical role in structuring the compositions throughout" [103]. Whether produced digitally (Ikeda's Test Patterns, 2008), or physically (Messier's Sewing Machine Orchestra, 2011), they feel cold, stark, mechanical, and binary; this is what Sangild calls "an almost inhuman gesture", which he contrasts to "Oval's quasi-organic syntheses" [25]. The lack of sentiment that these works convey is further reinforced through their rejection of melodic and harmonic material, and projected through their inorganic feel. Revealing his interest in "dark sounds" and "very basic harmonic systems", Bender, for example, describes his compositional process as a mechanical structure in which all the elements function just to bring the rhythm forward [97]. Even when using sophisticated technologies, the conventionally non-musical sonic material used in these works are 
conveyed in a raw and basic form: Bernier's Frequencies (a) (2012), regardless of its use of mechatronics and microcontroller programming, is composed for a number of sinetones and tuning forks, confirming his fascination with basic sound-generating units [104]. Aurally, visually, or audiovisually, these works reveal the materiality of their materials. In Cyclo, Nicolai and Ikeda's sound waves are visually expressed through oscilloscopes, whereas Zimoun's installation works are even titled after their materials: such as 80 prepared do motors, cotton balls, cardboard boxes $71 \times 71 \times 71 \mathrm{~cm}$ (2011). Accordingly, the direct and upfront nature of these works hides nothing: sinewaves are presented as sinewaves, filtered white-noise as filtered white-noise, DC motors as DC motors, and sewing machines as sewing machines. In this way, there is no subsumption of the material used within a representational or developmental "language", as materials are employed for their own inherent qualities and functions, and not articulated by a governing musical "discourse". By expressing raw materials in these bare and reduced forms, these works create a sensory experience out of audiovisual elements normally understood as unpleasant, uninteresting, and ignored or extraneous, which are now contextualised and regulated as macro and micro units, contained by arrays, grids, and repetition.

Regardless of the medium or mode of presentation, such works can be described as radically reduced systems that are stripped-down to a set of raw quantised sonic or visual elements, which although perhaps uninteresting in isolation, are compelling as a grouped and organised phenomenon (see Figure 5-1).

The rigorous minimalistic approach evident in these works, in addition to their emphasis on the visual appearance and physical existence of their material, equips them with Landy's "hold onto factor" ${ }^{26}$ discussed in Chapter 1 [18] . Through reduction, repetition, and clarity of sonic and visual texture and form, these works are readily accessible at one level, even if at other levels their materials are not conventionally musical or "beautiful". This juxtaposition grants such works their unique affectivity and

\footnotetext{
26 To reiterate, the "something to hold onto" factor ranges from certain sonic attributes and visual accompaniments that make works more accessible, to conceptual or visceral hints to which the audience can relate. Works highlighted in this thesis often employ several of Landy's hold onto factors such as homogeneity of sound material, clarity of texture, conventional use of rhythm, and presentation in audiovisual settings.
} 
aesthetic, which could also be considered as a balanced tension, a frisson, or an aestheticisation of the "ugly".

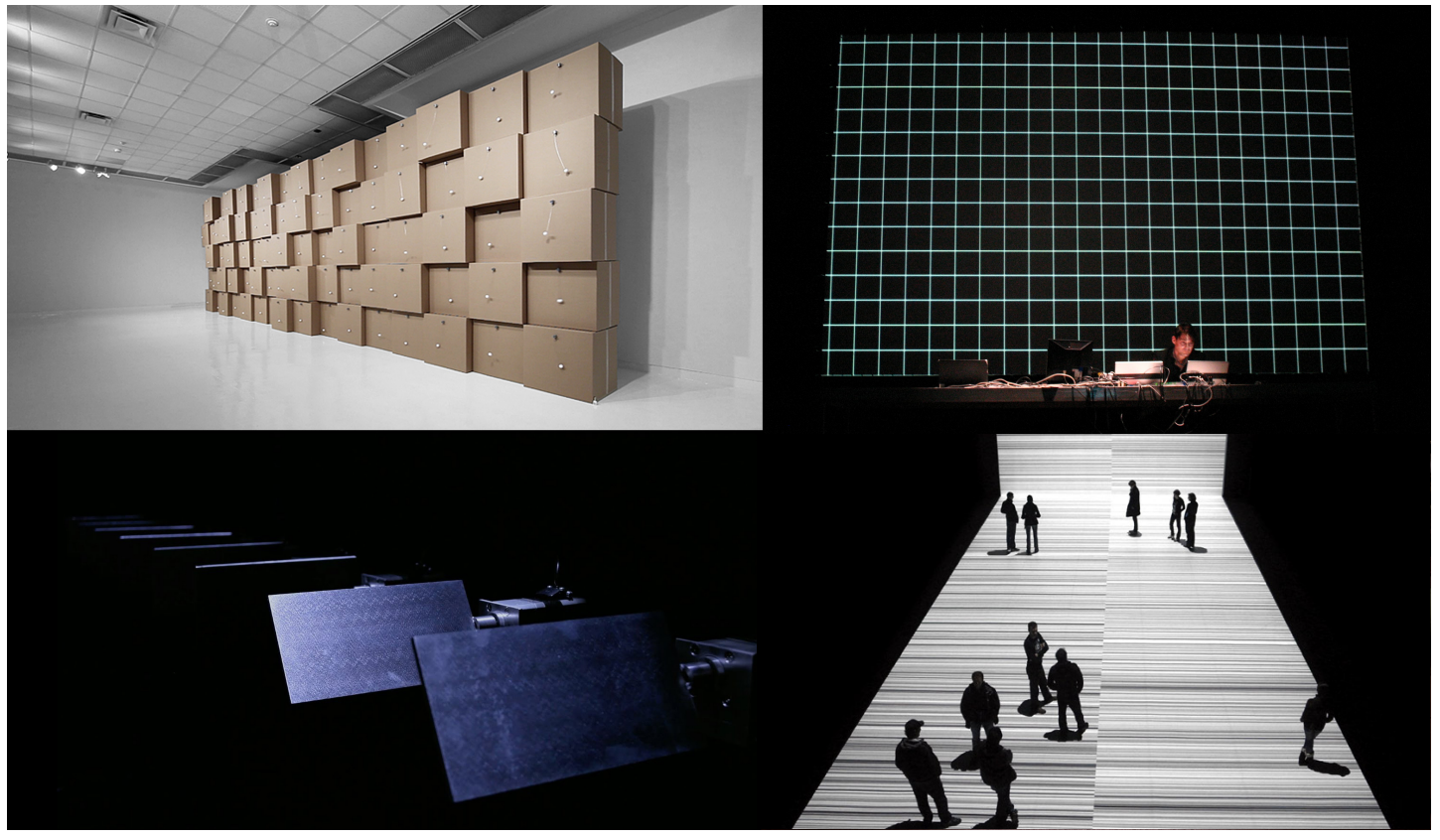

Figure 5-1. Top-left: 80 prepared dc motors, cotton balls, cardboard boxes $71 \times 71 \times 71 \mathrm{~cm}$ (2011) by Zimoun. Photo courtesy of the artist. Top-right: Olaf Bender aka Byetone performing live (2008). Photo by Alvaro Fárfan, via www.flickr.com (CC BY-NC 2.0). Bottom-left: Motor Music Test 55 (2013) by Daito Manabe. Photo courtesy of the artist. Bottom-right: test pattern [n 3] (2010), photo by Marc Domage, courtesy of Ryoji Ikeda.

While such aesthetic principles link these works to the author's compositions presented in the previous chapter, a line can be drawn between the two. Regardless of the aesthetic similarities in terms of output, Rasping Music, complex, and NOISE SQUARE all build upon conceptual and technical approaches with a firm foundation in academic research that, through their focus on grid-based patterning or noise as sonic material, enable realisation of the compositional objectives. In the works discussed in this chapter, however, it is rare to find any underlying narrative that is not primarily concerned with the sensory and formal experience of the material at the output. Here, the exclusive emphasis on the material and the grid at all layers does not require the same degree of conceptual and technical integration of materials, methods, and experiential output that is evident in the author's work. 
With these points in mind, here we begin to broach another, contextual and historical, link between these works, to which the author deeply relates: their relationship to Brutalism.

\subsection{Brutalism: from Architecture to Sound Art}

\subsubsection{Brutalist Architecture: an Overview}

A post-war movement in architecture, Brutalism (originally known as New Brutalism) emerged in the 1950s. Reyner Banham coined the term 'The New Brutalism', as “a deliberate attempt to construe [Alison and Peter] Smithsons' aesthetics as a new movement that would overcome the soft modernism of The Architectural Review's 'New Humanism,' or 'New Empiricism"' [105]. The origin of the term, however, was used equivocally by Banham, as it had multiple references: Le Corbusier's béton brut (raw concrete), neo-brutalist as an inside joke used by Hans Asplund in reference to the work of some of his Swedish colleagues, or Peter Smithson's nickname 'Brutus' [106]. Nevertheless, when first theorising the movement in 1955, Banham clearly defines Brutalism's chief principles as "memorability as an image, clear exhibition of structure, and valuation of materials 'as found"' [107]. In explaining Brutalism's mission, Banham argues that it is an amalgam of sets of stylistic features (as are found in Cubism) and ideological principles (typified by Futurism) [107]. The relevance of this to the preceding discussion should be clear, but before turning to these links, a little more historical and aesthetic information is first required.

Among the first architects to associate themselves with the term Brutalist were Alison and Peter Smithson. They describe Brutalism as a movement that "tries to face up to a mass-production society, and drag a rough poetry out of the confused and powerful forces which are at work [in it]", claiming that Brutalism's essence is ethical rather than stylistic [108]. Michael J. Lewis describes early Brutalism as the late appearance of modernism in British architecture, which "had been delayed by the Depression, world war, and England's incorrigible provincialism" [109]. The use of unpolished, unsanded, 
and unpainted wood and metal in the Smithsons' work, he argues, was inspired by Le Corbusier's rough-cast concrete in his Unité d'habitation: a monumental emblem of modernist architecture. By associating their work with the brut of Le Corbusier, as Lewis states, early Brutalists wanted to express their Brutalism as having an "impeccable modernist pedigree" [109]. He argues that the radical transformations of modernist painting and sculpture from 1930s to 1950s had not yet left any appreciable mark on modern architecture, which was still in alignment with the "laconic, Platonic, and coolly functionalist" modernism of the 1920s. Therefore, as a late but radical leap in modern architecture, "the rise of the New Brutalism was an expression of this discrepancy in sensibilities, and the new appetite for coarse textures and raw materials" [109]. As Banham importantly points out, "what characterizes the New Brutalism in architecture ... is precisely its brutality, its je-m'en-foutisme, its bloody-mindedness" [107].

John MacArthur argues that Banham's goal in 1955 was “defining Brutalism as a movement ... in the expectation that it could then become an observable style" [110]. Whatever the intentions of Banham and the Smithsons, and their ideological motivations, Brutalism today is understood not for its ethical dimension, but aesthetically as an architectural style whose appearance can be described as austere, stark, cold, and monolithic, with these primary features created through strict geometries, repetitive modules, and undisguised use of materials (see Figure 5-2). Certainly, anti-beauty "is the one characteristic of Brutalism that has by no means been lost on the public" [111]. This refers to Banham's definition, "anti-beauty in the classical aesthetic sense of the word", that was a reflection of the broader "anti-academic aesthetics" movement at the time [107], one that was "emerging in the era of "angry young men", in literature, theatre, film and musique concrète" [112]. In this way, Banham's Brutalism "sought to provoke rather than to please the senses" [113]. This is perhaps why "Brutalism never caught on with the public, who could not be made to see that the problem with modern architecture was that it was insufficiently surly" [109]. 


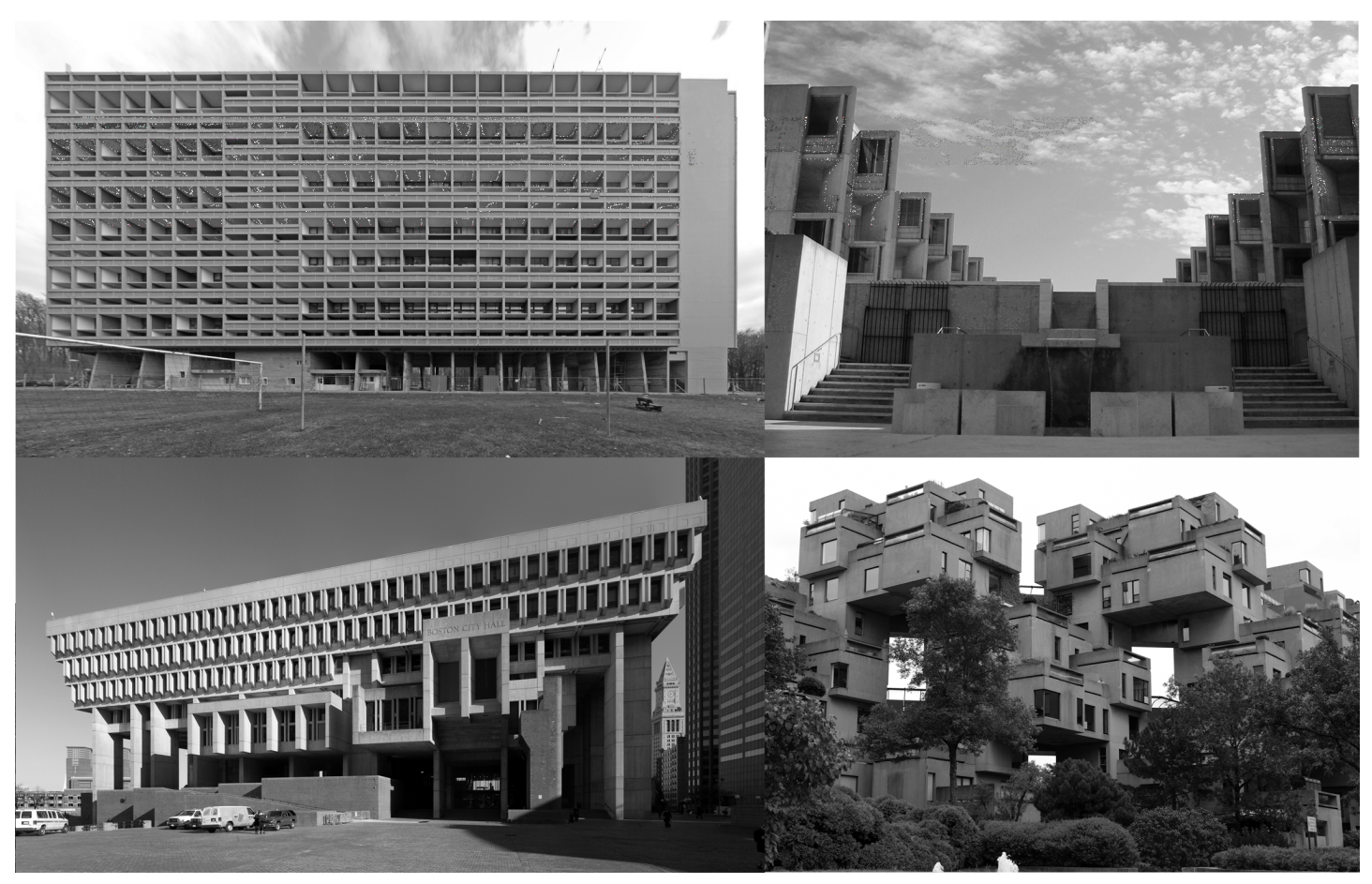

Figure 5-2. Top-left: Unité d'habitation by Le Corbusier. Photo by Sébastian Pérez-Duarte, via www.flickr.com (CC BY-SA 2.0). Top-right: Salk Institute by Louis Kahn. Photo by Jim Harper, via Wikimedia Commons (CC SA 1.0). Bottom-left: Boston City Hall by Gerhard Kallmann and Michael McKinnell. Photo by Daniel Schwen, via Wikimedia Commons (CC BY-SA 4.0).

Bottom-right: Habitat 67 by Moshe Safdie. Photo by Wladyslaw Sojka, via Wikimedia Commons (CC BY-SA 3.0).

The utilisation of raw materials as they are found (that is unprocessed, unrefined, and undecorated) is evidence of the minimalistic aspect of the Brutalist aesthetic. For example, Fabrizio Gallanti refers to works of Sigurd Lewerentz as "construction stripped to the bone, reduced to its minimal functional terms, where elements of architecture are understood for what they make" [114]. Likewise, Smithsons' Hunstanton School "appears to be made of glass, brick, steel and concrete, and is in fact made of glass, brick, steel, and concrete" [107]. However, even if we regard Banham's ethical Brutalism as historical and not extendable to a popular understanding of Brutalism, at a stylistic level Brutalism is still very much defined by a predominance of material and formal features which are minimalistic: extensive use of right angles, cubes, abstract geometric patterns with forms created through use of repeated units, a monochromatic or very reduced colour palette, a thorough eschewing of decorativeness, and exposed use of raw (that is found) materials. 
Such an architectural aesthetic formed a strong personal context for the author's later reception of the sound-based artworks discussed in this thesis. Born and raised in Ekbatan, a Brutalist residential building complex in west Tehran built in the 1970s, the author developed a sense of attachment to the "anti-beauty" aesthetics of the cold and austere blocks of concrete, stacked in perfectly aligned geometric arrays, that were the site of his upbringing (Figure $5-3) .^{27}$

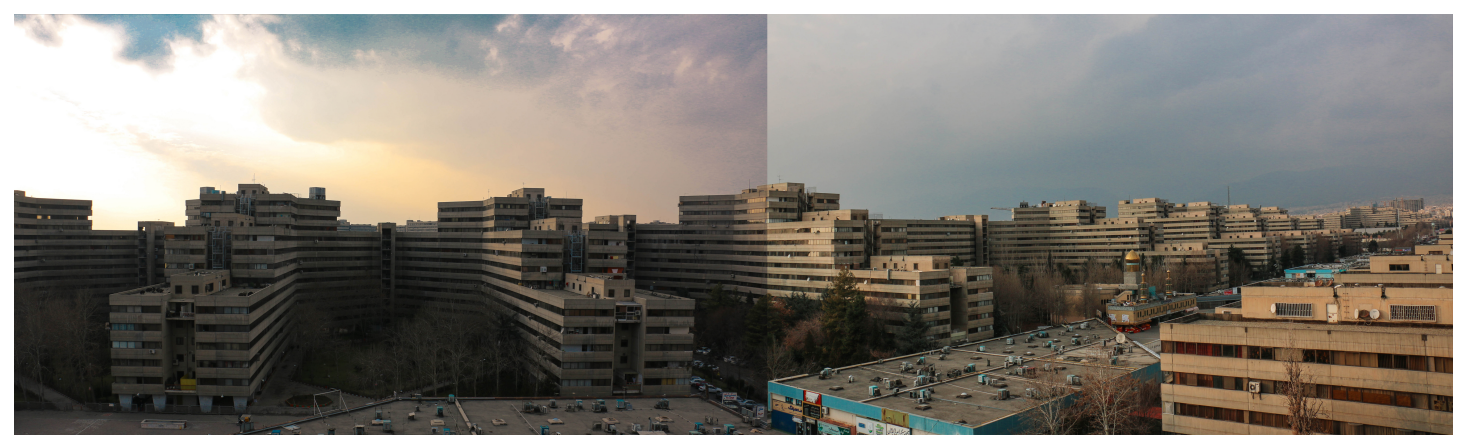

Figure 5-3. Ekbatan residential building complex, Tehran. Photo courtesy of Hoda Zareei.

Such deep-rooted topophilic affinity for the rigidly structured harshness only recently became apparent while investigating the intersecting aesthetic features that held strong appeal for the author. Further investigation into Brutalism made the connection between the work undertaken by this research and Brutalist aesthetics clear: reductionist, minimalist, and functional qualities which provide form for the provocative anti-beauty aesthetic in which noise replaces the concrete and other physical materials of Brutalism, and its spatial grid is expanded to the temporal domain.

\footnotetext{
27 This can be explained as a form of topophilia: an ecopyschological term describing a person's affinity for their environment.
} 


\subsubsection{Brutalist Sound Art: Realisation}

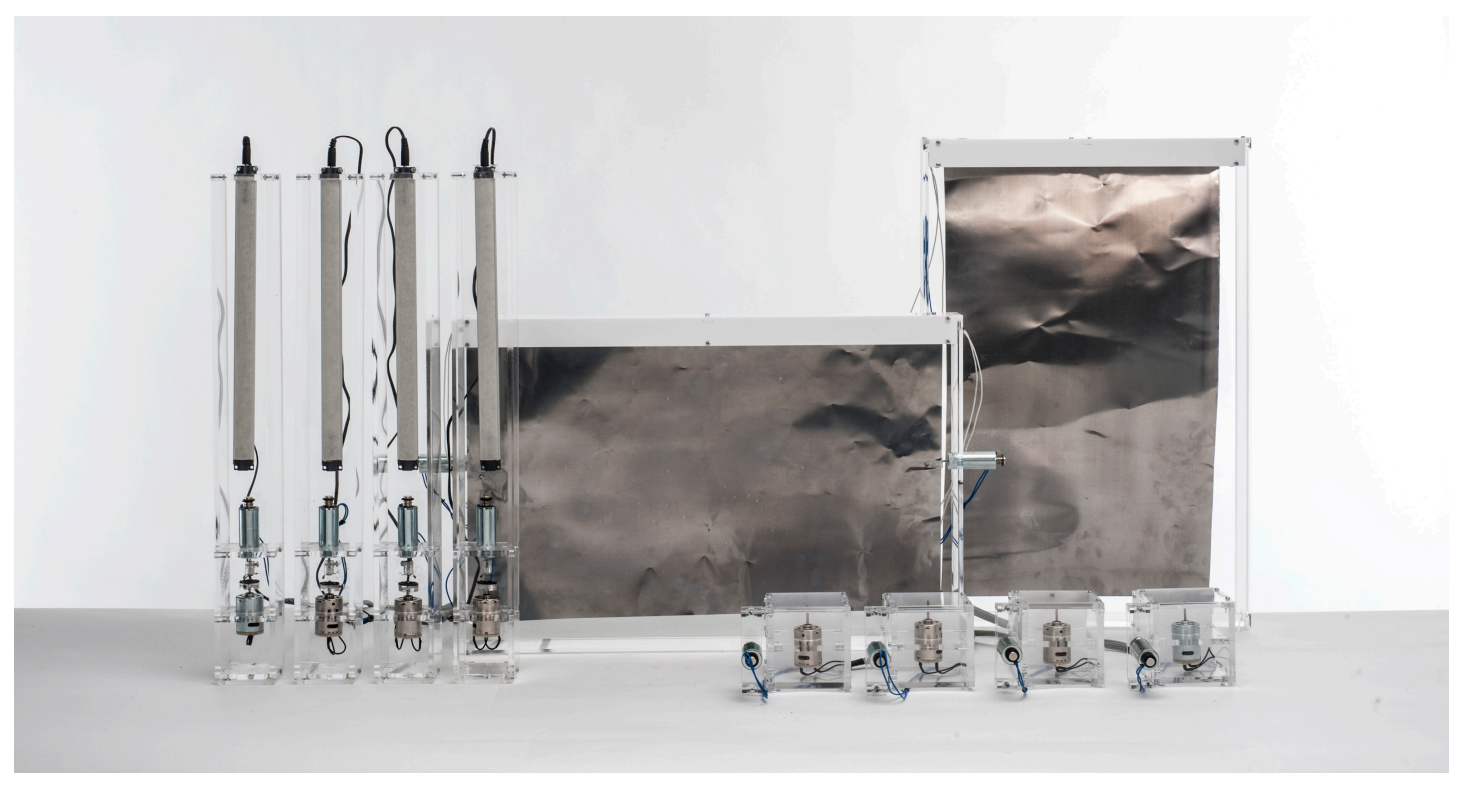

Figure 5-4. The ensemble of mechatronic sound-sculptures.

Over the course of this research, an ensemble of ten mechatronic sound-sculptures was developed (Figure 5-4). As discussed in Chapter 3, based on their specific sonic and technical features, these sound-sculptures are grouped into three instrument-types: Rasper $(\times 4)$, Rippler $(\times 2)$, and Mutor (×4). Although each instrument uses a different soundgenerating apparatus and has its own sonic and visual characteristics, they all share aesthetic aspects that links them closely to Brutalism.

All three instruments employ non-musical objects whose sonic aspects are conventionally perceived as "non-beautiful": the buzzes, whirs, and clicks and clacks of electromechanical objects, the sounding of bits and pieces of metal. Based on the arguments provided in the previous section, the Brutalist aesthetic has, with each subsequent sound-sculpture, assumed a more central role. In Rasper, the mechatronic components, although as exposed as possible, have been put together with a number of other materials in order to construct a certain mechanism, that is to serve a purpose that is extraneous to their inherent quality. In Rippler, the mechanism is simplified for the purpose of emphasising one of the mechatronic component's intrinsic features, where in 
Mutor, the mechatronic component appears, both visually and sonically, in the most direct and untreated way, as found.

By presenting its "non-beautiful" material in sculpture forms, the ensemble takes them through an aesthetic transformation, in which the "ugly" sonic by-product of everyday technological life is turned into a medium for artistic expression. This transformation is completed with the help of mechatronic techniques and microcontroller programming, which affords the instruments the ability to create exactly controlled and repetitive patterns and motions. By expressing but regulating the sound of the instruments' raw material in terms of rhythm and timbre, and structuring them through strictly metric rhythmic patterns, the aggregation of these sound-sculptures as an ensemble affords the composition of a temporal grid of noise constructed from various timbral units and rhythmic layers. In doing so, what is normally hidden inside the black box of a machine is brought back to the foreground in a fully visible and audible form, and expressed as found, that is undecorated and unmodified. Enclosed in entirely transparent structures, the materiality and corporeal being of all the components are clearly exhibited, exposing their usually covert (but always potential) aesthetic essence. Limited to right angles, cubes, squares, and rectangles, the enclosures are designed for function alone, leaving no room for ornamental features in the sculptures' reductionist, minimalist, and strikingly bare appearance. This austere visual quality is further highlighted through bursts of fluorescent light, which in negotiating a fine line between the mesmerising and the uncomfortable, intensify the sensory experience of the works in a bold audiovisual manner.

Using identical mechatronic components across all three instrument-types (that is the same types of DC motors and linear actuators) creates further homogeneity when they are collected as an ensemble, in keeping with the rigour of the Brutalist ethos. In doing so, the work simultaneously undertakes a sonic transcoding of these principles that is expressed through the use of the intrinsic noise of its materials, and the clear and immediate comprehensibility of its sonic structure. A sonic equivalent of the visual memorability of Brutalist architecture, this audible structure is established through the use of a steady-state sonic palette and purely grid-based rhythms. Repetition is 
incorporated as a key formal element, which eliminates organic formal development from the work, while the utilisation of mechanical noise as the only sound material functions as a sonic equivalent to the anti-beauty qualities of Brutalism.

Building upon these aesthetic links and in order to realise the idea in an explicit manner, the following works were developed, with a mind to realising Brutalist principles in a series of sound-based works.

\subsubsection{The Brutalist Noise Ensemble}

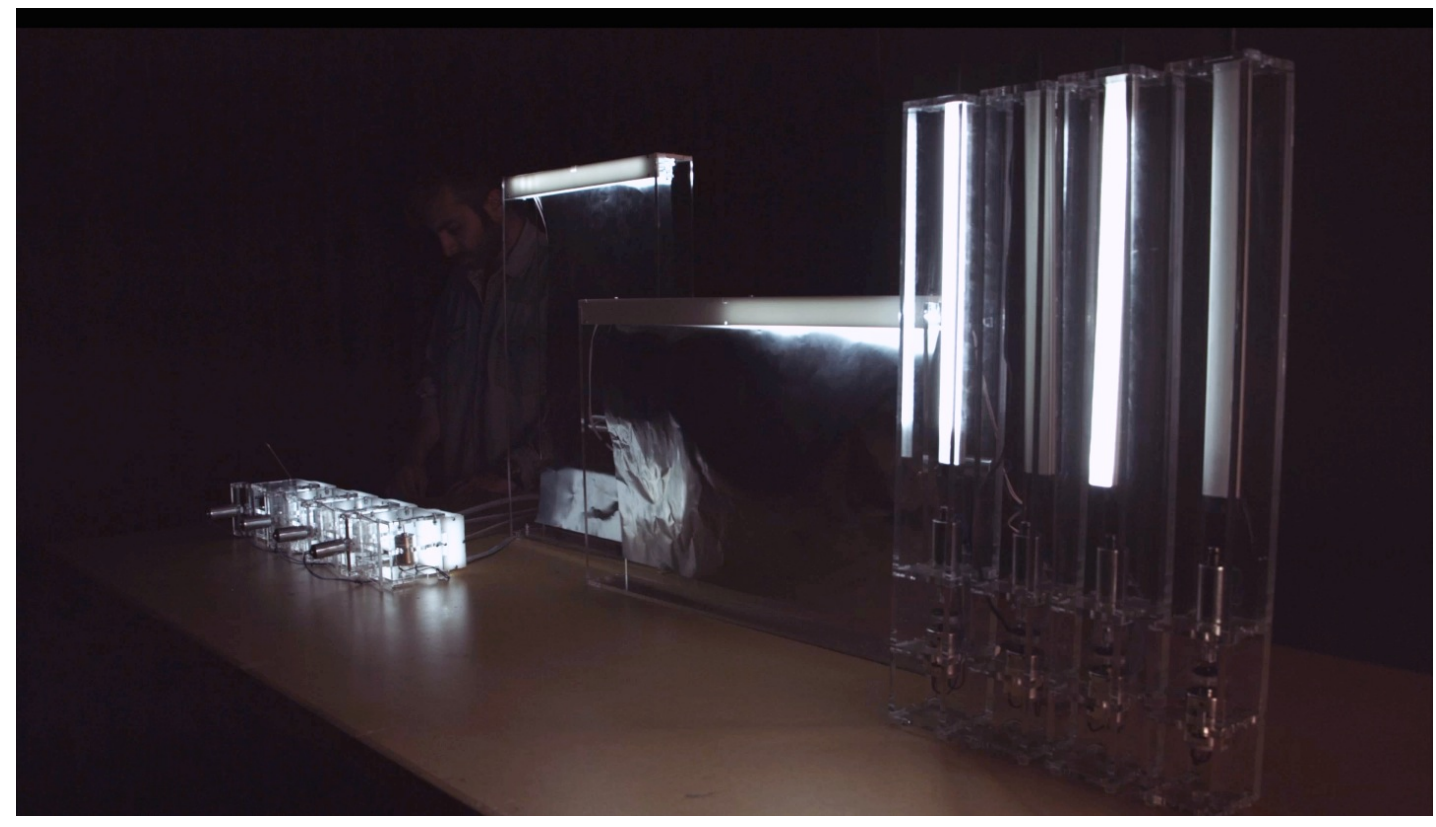

Figure 5-5. The Brutalist Noise Ensemble (2015), audiovisual performance.

The Brutalist Noise Ensemble (2015) is an audiovisual performance composed for the full ensemble of mechatronic sound-sculptures (Figure 5-5). The piece comprises three sections that are separated by short transition passages. All three sections are structured on a series of rhythmic patterns through which the three instrument-types interlock, with the transitions accomplished by a solo instrument-type. The rhythmic patterns are metric and repetition-based and the piece develops very gradually, as each section sequences through them via four, eight, 16, or 32-bar long loops (see Figure 5-6). 


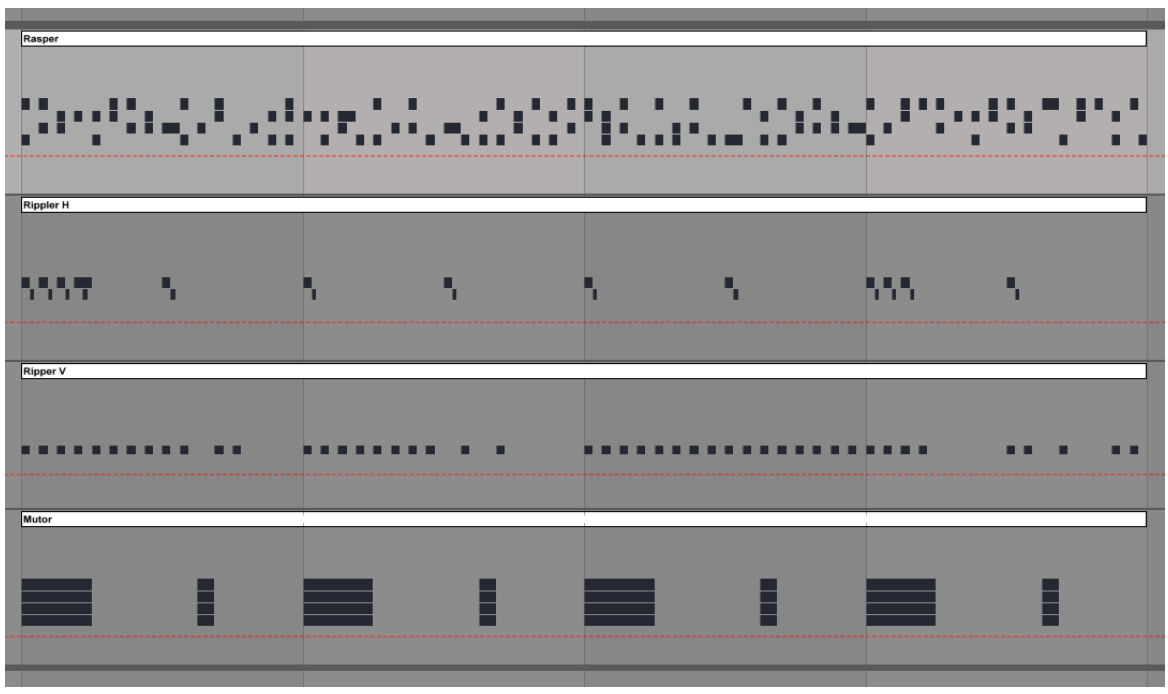

Figure 5-6. The Brutalist Noise Ensemble: MIDI notation of a 4-bar long rhythmic pattern composed for Rasper (top), Rippler $\mathrm{H}$ and $\mathrm{V}$ models (middle), and Mutor (bottom).

Although the sections differ rhythmically and timbrally, this difference is deliberately minimised in order to avoid a developmental form and preserve the linear temporal dynamic of the work. During the performance, the performer is in charge of navigating through different patterns and sections, while controlling the work's timbral elements such as the speed of the motors in Mutors or the solenoid stroke length in Ripplers.

\section{STREAMING MUSEUM about exhibitions+ IDEACLoud news visit collect follow a}

\section{CURRENT}

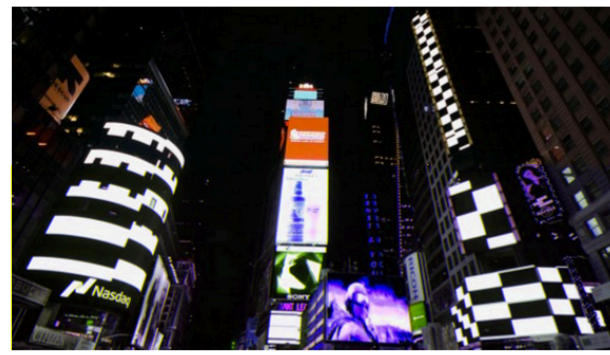

RYOJI IKEDA, Japan's leading electronic composer \& visual artist, takes over Times Square with "test pattern [times square]". Video by Streaming Museum.

[Video] Times Square Midnight Moment
PAST $\rightarrow$

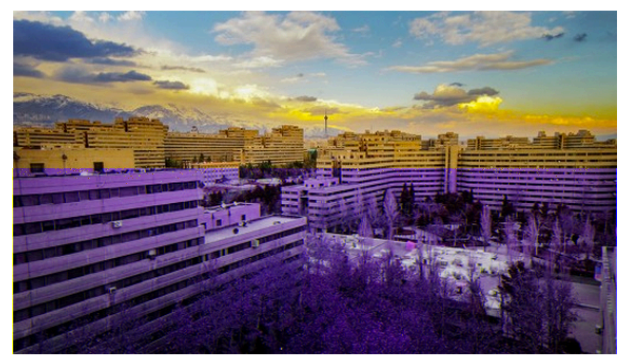

The Story and Kinetic Sound Sculptures of Mo H. Zareei, his Brutalist Noise Ensemble and the Artists and Brutalist Architecture in Tehran that Influenced Him

[Videos \& Gallery] Includes Kinetic art of Trimpin, Bernier, Messier, Zimoun, and Pe Lang.

Figure 5-7. The Brutalist Noise Ensemble curated by Streaming Museum (a screen shot of Streaming Museum website in March 2015). 
The Brutalist Noise Ensemble was curated by Streaming Museum ${ }^{28}$ (Figure 5-7), promoted by Composers Now ${ }^{29}$ during the Composers Now Festival 2015 in New York, and described as "an electrifying musical performance" on Atmel website ${ }^{30}$.

The performance was premiered at Toi Ponēke Art Gallery in Wellington, as part of the Low Noise Vol. 2 exhibition in March 2015, which resulted in an interview with SOUNZ, Centre for New Zealand Music ${ }^{31}$.

\subsubsection{2 machine brut(e)}

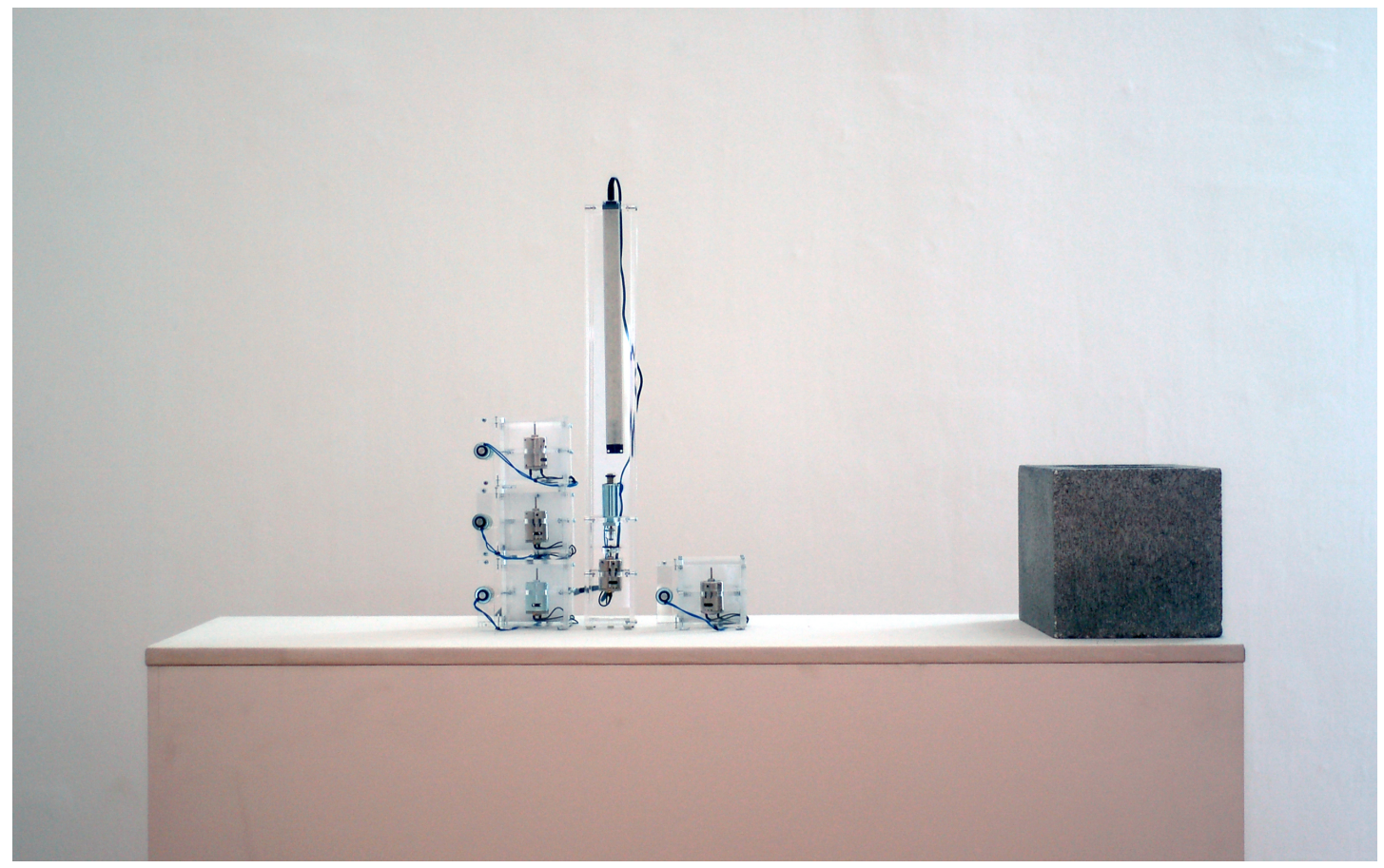

Figure 5-8. machine brut(e), composition0111. Photo by Jim Murphy.

machine brut(e) (Figure 5-10) is a collection of ten installation pieces composed for Rasper, Rippler, and Mutor. Each piece incorporates a different combination of the soundsculptures and is composed as a one or 2-bar long pattern that goes through an endless loop. Strictly metric and pulse-based rhythms are used as a sonic metaphor for the gridbased and geometric structure of Brutalist architecture. By using nonstop repetitions of

\footnotetext{
${ }^{28}$ Further documentation available at: http://www.streamingmuseum.org/mh-zareei

${ }^{29} \mathrm{http}: / /$ www.composersnow.org

30 http://blog.atmel.com/2015/05/19/sound-sculptures-put-on-an-electrifying-steel-drum-performance

31 http://sounz.org.nz/resources/show/1264
} 
one or 2-bar long loops, the piece draws attention to the very essence of its constituent sonic material and every detail and nuance of their noise, in parallel to the validation of material in Brutalist architecture ${ }^{32}$. In this way, the non-developmental linearity of the previous work is taken to an even more extreme point in order to create a sense of temporal monolithism that matches the block-like aesthetics of Brutalism. In addition, this constant repetition helps convey an instant audible structure that links the work to the memorable imagery of Brutalist buildings. As shown in Figure 5-9, the physical placement and distribution of the sound-sculptures in each composition has also been taken into account meticulously, abiding by the strict geometries and highly ordered visual aesthetic of Brutalism. Therefore, each piece is developed not only as a work of sound art, but also a sculptural composition. To further highlight the visual Brutalist aesthetic, a block of raw concrete (béton brut) is emblematically featured in all ten compositions.

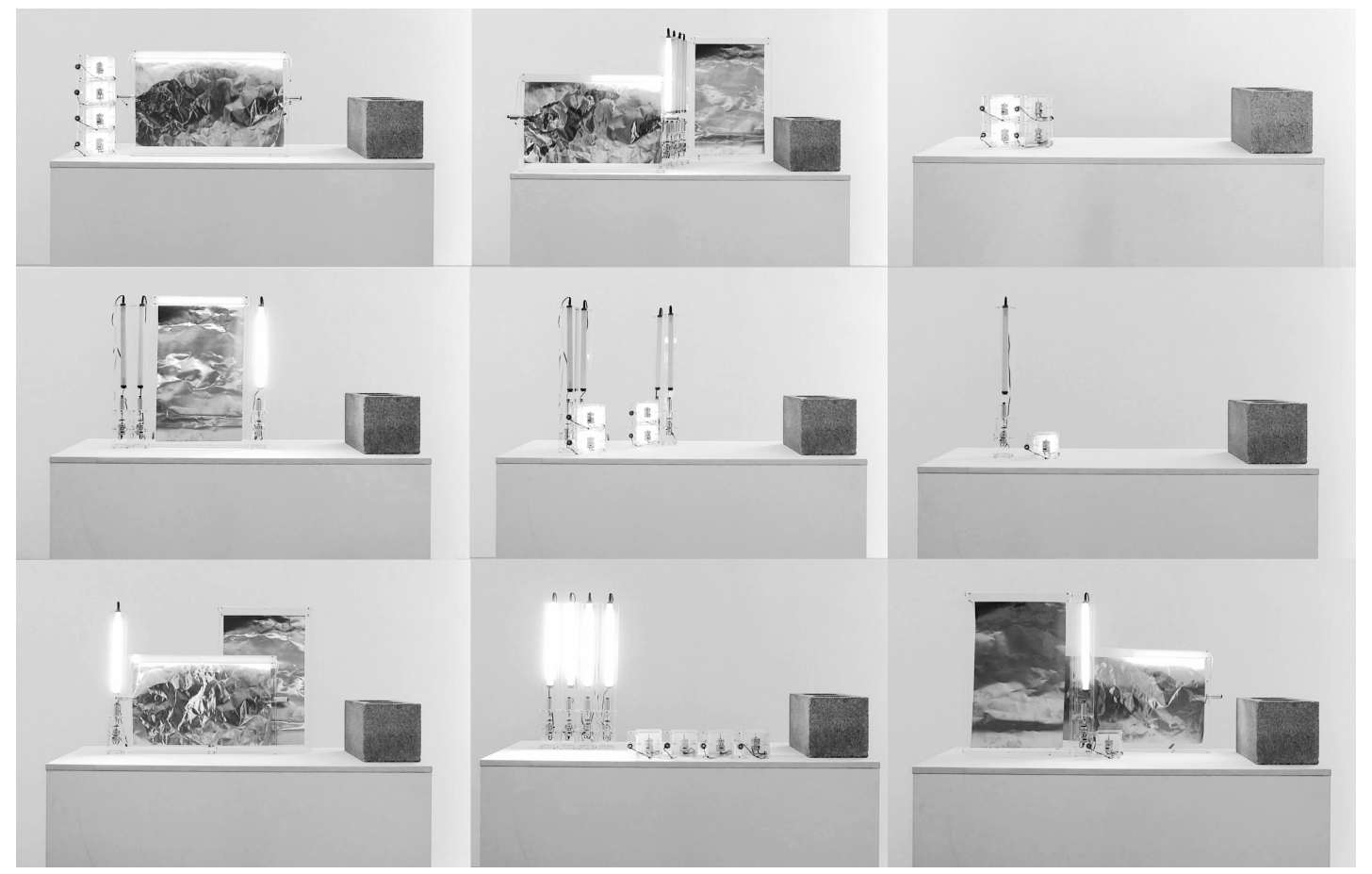

Figure 5-9. machine brut(e): nine out of ten compositions.

32 Here, a connection can be made between this strategy and Pierre Schaeffer's use of loop (locked groove) in drawing attention to the sound itself. 
A video compilation of machine brut(e) installed at Adam Art Gallery in Wellington was exhibited in the Plugin New Media section of the Contemporary Istanbul 2015 International Art fair. The video was also featured on Creative Applications and Fast Company Design websites ${ }^{33}$. As seen in Figure 5-10, the ten compositions are titled using binary numbers, emphasising the pulse and grid-based aesthetic of the work.

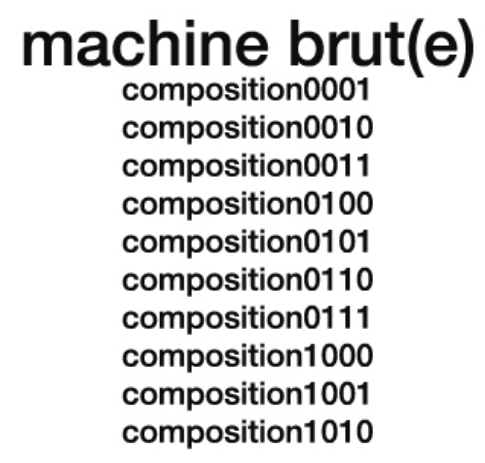

Figure 5-10. machine brut(e): series artwork.

"Much like the music of IDM masters Autechre, there isn't simply beauty in the repetition and geometry of the audio, but in the tonal qualities of the sound. So, much like Brutalist architecture, there is a depth to Zareei's machine brut(e) that isn't immediately accessible, but most certainly there".

- The Creators Project

"When Zareei's instruments play together, their noise is harsh and propulsive, sounding like a warehouse rave for robots. The strict grid-like structure of the music stays true to brutalist architecture, known for its ordered pragmatism".

- Fast Company Design

${ }^{33}$ Video documentation of machine brut(e) available at: $\underline{\text { https://vimeo.com/127683583 }}$ 


\subsection{Discussion: Sound-based Brutalism}

Can we now take the proposed connection a step further and expand the realm of sound-based brutalism to the broader context in which The Brutalist Noise Ensemble and machine brut(e) have been situated, one that includes the compositions presented in Chapter 4, as well as various contemporary works discussed in this chapter? To make the claim that certain contemporary aesthetic trends in sonic arts are influenced by or directly correlated to a - primarily architectural - movement that started sixty years ago and was deemed to have failed within a few decades, is at first glance rather challenging. The original (New) Brutalism arrived in 1950s and is supposedly well past its prime. For a number of decades now, the movement has been regarded as a failure by many, harshly criticised by critics and widely disdained by the public, to the extent that a substantial number of Brutalist buildings have been demolished over the past few decades [115][116][117]. The rejection of Brutalist architecture has several roots, including its starkly alien visual presence with regard to its surroundings [118]. But it is most importantly the "anti-beauty" aesthetics of Brutalism that fails to appeal to the public [111], and which give rise to derisive names for Brutalist buildings, such as "concrete monstrosity" [112].

However, it should be made clear that it is not the intention of this thesis to argue for the direct translation of certain architectural principles as a set of criteria for creating sound-based artworks, as for example in Xenakis' adaptation of architectural models in the composition of his early music [119]. Nor is the goal of this discussion to declare the birth of an entirely new practice in sound-based arts. The goal here is to establish a frame of reference, built upon a shared set of aesthetic features that are most cogently expressed in an architectural form - Brutalism - and which can be used to draw together and draw attention to a set of sound-based artworks across genres and media. Having encountered incoherent neologisms such as "Raster-Noton-y" or "Zimoun-ish" in a colleague's or an audience member's reference to works with similar aesthetic qualities, the author argues that this loose sense of an aesthetic has become a broad aesthetic movement which, once defined as such, becomes more amenable to further 
investigation, definition, and clarification. With this in mind, and based on the arguments made above, the author suggests the term brutalist as an appropriate focal term. In doing so, and to re-iterate, this is not an argument for this set of sound-based works having direct connections to Brutalist architecture. Rather, we suggest "brutalism" as an apt descriptor for their shared aesthetic principles, as well as providing an historical point of reference which is decoupled from the normative, and historically more removed, association of noise-based practices with Futurism. In this way, Brutalism's revival can be extended to the realm of sound, as an extreme manifestation of modernist aesthetics expressed in the works discussed here: uncompromising in their use of the brut of postdigital noise, modern technological objects and artefacts, and the presentation of these through stripped-back forms and grid-based structures.

In an effort to "delineate one genre that highlights a conceptualisation of sonic matter", Sonya Hofer points to the insistence on materiality through the "conception of sound as particles" in microsound [98]. More broadly, Hegarty describes noise music as "an attempt to reassert the material over the musical" [120]. With these points in mind, sound-based brutalism encompasses sound-based works which focus on the materiality of their "anti-beautiful" materials in sonic - and often also visual - forms, through a highly ordered, organised, and often quantised mode of expression. Indeed, it could be argued that sound-based brutalism embraces Pierre Schaeffer's objet sonore through its focus on basic sound-objects, but rejects his concept of reduced listening through emphasis on the material thingness of the object itself. ${ }^{34}$ If the Brutalism of Banham tried to "confound [Cubism and Futurism] and belong to both at once" [107], sound-based brutalism feeds on the noise of Futurism and the reductionist rigour of minimalism - a child of Cubism - at the same time, taking its raw material from the former and sculpting it through the latter.

\footnotetext{
34 The link to musique concrète, while an interesting one, is not pursued here given the complexities introduced by Pierre Schaeffer's theorising of sonic objects, and in particular the intentional transformation of these from a raw to a purified state through reduced listening, in order to make them amenable to musical use. This, it could be argued, is a form of aestheticisation at odds with the ethos and aesthetics of Brutalism.
} 
Beyond this, and as stated in a recent $B B C$ documentary on Brutalism, "half a century after its heyday, a wholesale rehabilitation of Brutalism is on the way" [121]. In the concluding section of his thorough examination of British Brutalism, Alexander Clement claims that "in spite of the debates about the aesthetic qualities of many period Brutalist buildings, the style has been revived in more recent years" [122], and according to Michael J. Lewis, "it seems safe to say that there is no topic in architecture at present that is of greater interest and curiosity than Brutalism" [109]. ${ }^{35}$ Indeed, this BBC documentary on Brutalism concludes with the announcement that "we are witnessing the emergence of Neo-Brutalist architecture", and introduces a number of visual artists who are integrating Brutalism into their work. As has been argued here, the shared aesthetic features of the work of a broad range of artists and musicians warrants the inclusion of sound-based practices in this neo-brutalism.

\section{The findings of this chapter are featured in the following publications:}

Mo H. Zareei, Dale A. Carnegie, Ajay Kapur. "Physical Glitch Music: A Brutalist Noise Ensemble," Leonardo Music Journal 25 (2015).

Mo H. Zareei, Dugal McKinnon, Dale A. Carnegie, and Ajay Kapur. "Sound-based Brutalism: an Emergent Aesthetic," Organised Sound 21(1) (2016).

35 Christopher Beanland's recent article in Independent (2014), Jonathan Clancey's article on BBC (2014), the recent issue of CLOG magazine (February 2013) on Brutalism that this document has been referring to repeatedly, and Barnabas Calder's upcoming publication (2016) are all heralding this revival as well. 
131 
132 


\section{Chapter 6

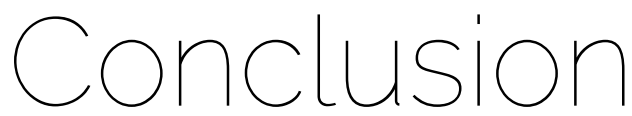

This chapter provides a summary of the thesis scope, discusses future work, and presents the novel contributions that have arisen from this research.

\subsection{Summary}

\subsubsection{Motivation / Objectives}

The overarching goal of this research is the exploration of the potential aesthetics of the mechanically produced noise characterising the modern urban soundscape. Accordingly, and aligned with Landy's ideas on the accessibility of the works of organised sound discussed in Chapter 1, two primary strategies were implemented to boost the sensory perception of sounds that are normally considered mundane, unpleasant, or extramusical. First, a grid of metric and pulse-based patterns was used to establish what Landy refers to as a "hold onto factor", or in Attali's words, a decipherable "code" in channelisation of noise. Second, an audiovisual context to highlight the physical existence of the noise was employed as the mode of presentation. Based on the arguments provided in Chapter 1, mechatronic sound-sculpture was chosen as the suitable medium to fulfil the objectives. 


\subsubsection{Background / Related Works}

As cited in Chapter 2, the number of works of sound art and music that incorporate mechatronics and robotics has significantly increased over the past few decades. Regardless of the specific apparatus they employ, a large number of these works are rather deterministic systems, most often inspired by some already-existing musical instruments, whose goal is to achieve a familiar musical output. We identified these works as 'musical robotics' and described them as mechatronic versions of conventional musical instruments with automated, modified, or extended capabilities, where a machine substitutes for the action of the human performer in, for example, plucking a guitar string. As it was argued, here, the inherent actuation noise of the mechatronic components raises an issue and needs to be overcome, either through various dampening techniques, or amplification of the musical instrument's sound. Therefore, in this scenario, while the technological medium provides the means to achieve the desired sound, it simultaneously introduces into the transmitting musical signal an undesirable noise that has to be attenuated. Accordingly, if "a glitch is that which betrays the fidelity of the musical work" [123], the inbuilt noise of the mechatronic machines was a physicallygenerated counterpart for the skipping $\mathrm{CD}$ or speaker distortion in the realm of digital sound. From the viewpoint of glitch however, this - otherwise undesired - noise could open a door to new sonic material, and be transformed from a subordinate by-product into the primary content, in formation of an aesthetic construct. This aesthetic recycling of mechatronic noise is in fact the chief principle behind this research. Given the use of metric and grid-based rhythms in aestheticising the noises of digital technologies in the examples of laptop-produced works generally known as glitch, the output of this research in fact expands the boundaries of glitch music into the physical realm.

In order to realise this idea, a series of ten mechatronic sound-sculptures were designed and developed.

\subsubsection{Design / Development}

The sound-sculptures developed over the course of this research are grouped into three different types: Rasper $(\times 4)$, Rippler $(\times 2)$, and Mutor $(\times 4)$. As introduced, discussed, and 
examined in Chapter 3, each type varies in terms of parts, mechanism, and sonic qualities. All three types are driven by the same custom-designed driver board and controlled using microcontroller programming. For all instruments, the communication is accomplished using MIDI messages.

Rasper, which was the first sound-sculpture in the series, comprises a DC motor attached to a disk, a piece of spring steel connected to a solenoid, and a fluorescent LED strip, that are all held together in a clear acrylic enclosure. Rasper's sound-generating mechanism is somewhat inspired by the one used in a number of Russolo's noiseintoners: the crank is replaced with the motor, the lever with the solenoid, and the vibrating material, that is the metal string, is replaced with the spring steel. As the solenoid pushes out, the motor spins the disk. The sound is generated when the sharp edge of the spring steel touches the rotating disk. By using different speeds of rotation, timbre and frequency of the sound can be changed. The LED strip is driven by the same signal as the solenoid. Therefore, every noise pulse is visually reflected through a burst of light.

Although the dominant sonic output of Rasper is caused by the contact between the spring steel and the disk, the solenoid's actuation noise and the buzzing of the motor also contribute to the resulting sound. Both of these were sources of inspiration for the design and construction of the succeeding sound-sculptures. Accordingly, Rippler's sound-generating mechanism is based on amplification of the solenoid's actuation noise through a thin sheet of steel. The instrument is designed in two different models (vertical and horizontal), depending on the orientation of the steel sheet and the direction of actuation. Both models comprise a steel sheet framed in a clear acrylic frame. While in the vertical model the sheet is attached to a single solenoid that is connected to it from the top, in the horizontal model, two solenoids mounted on two sides of the frame are connected to both ends of the sheet. When the signal is applied, the solenoids set the sheet into vibration. In this manner, the actuation noise of the solenoids is accentuated and amplified through the series of pulses caused by the movements of the sheet. In both models, the topside of the frame is a rectangular tube of fluorescent LEDs whose synchronous illumination reflects the movements of the solenoid and the sheet. 
Lastly, in Mutor, the external actuated object is removed and the sonic focus has shifted directly onto the noise of the mechatronic component itself, that is the motor. In this sound-sculpture, the noise of a DC motor that is placed in a clear acrylic box with a pivoting door, is controlled and modified in terms of volume and timbre. While the primary source of sound here is the buzzing of the motor - whose speed can be controlled, creating various tones - a solenoid mounted on the pivoting door is used to open and close it, changing the timbre and amplitude of the motor's sound. Similar to the previous sound-sculptures, an LED panel, which is mounted on the back of the box, provides visual feedback for the modulations in sound: as the solenoid pushes out and opens the door, the LED panel lights the entire box up.

An evaluation of the sound-sculptures' key sonic characteristics was provided in Chapter 3. Prior to their employment in the compositions discussed in Chapter 4, this information was used to provide a better understanding of the instruments and their various technical and performative capabilities. Based on the data acquired from a different set of tests, it was shown that the sonic output of all three instruments was acoustically noisy, and as a collective, they covered a wide range of frequencies from low-mid (Rippler), mid (Mutor), to high (Rasper) ranges.

\subsubsection{Application / Realisation}

The sound-sculptures were used in the creation of a number of new compositions that were discussed in Chapter 4. With these three pieces, it was intended to employ the sound-sculptures in compositional forms closely integrated with the aesthetic, ideological, and technical aspects of the instruments themselves.

The significance of metric and pulse-based rhythms in minimal music, in addition to the minimalistic design features of the sound-sculptures, led to the composition of Rasping Music, in which Steve Reich's Clapping Music is remodelled and modified for Rasper. The piece has been realised both as an installation (at Sonic Arts and Engineering Expo 2013, and Lux Festival 2014), and as a performance (at City Gallery Wellington and Adam Concert Room at New Zealand School of Music). Rasping Music won the $1^{\text {st }}$ Prize in the international Sonic Arts Award 2015 in Sound Art category. 
NOISE SQUARE was composed as an effort to fully emancipate the soundsculptures' programmability and autonomous capabilities. Among various generative and self-evolutionary systems, the grid-based structure of cellular automata and their minimalistic visual aesthetics made them a suitable candidate for an algorithmic composition using the mechatronic sound-sculptures. NOISE SQUARE comprised part of the ten percent of accepted artworks among approximately 1500 submissions to the International Symposium on Electronic Art 2015, and was realised at the Vancouver Art Gallery during the symposium.

Lastly, complex was an attempt to create an ideologically homogeneous work in which both inputs and outputs are inspired by contemporary urban noise. Using the Citygram database, the piece created a metaphorical aesthetic recycling of New York City's noisy soundscape by mapping the noise levels of four symbolic locations in Manhattan onto four mechatronic sound-sculptures. complex was realised during the International Conference on Auditory Display 2014 at NYU MAGNET in New York City, as one of the four installation pieces selected for the artistic programme of the conference.

In addition to these three pieces, the mechatronic sound-sculptures developed in this thesis were used in a number of other works including:

- Abrasive Stanza No. 1 (2014): an audiovisual performance using Rasper, featured in the international conference on New Interfaces for Musical Expression 2014 in London as the first piece among the four pieces selected for the NIME opening concert, and Tehran Digital Arts Expo 2014,

- Artifice (2014): an audiovisual performance using Rasper and Rippler, featured in the International Symposium on Electronic Art 2014 in Dubai, as one of the ten performances accepted to the artistic programme of the symposium,

- Gradient (2015): an audiovisual installation using Mutor, featured in Low Noise Vol. 2 exhibition at Toi Pōneke Gallery, Wellington, which led to a video interview with the Centre for New Zealand Music.

\subsubsection{Observation / Reflection}

Following the discussion on the different compositional platforms in which the mechatronic sound-sculptures were used to create new works, Chapter 5 took a step towards finding a common aesthetic ground across the work undertaken in this thesis, 
and the cross-medium examples of sound art and music discussed in Chapter 2. Accordingly, the last part of this thesis was dedicated to the formalisation of a growing aesthetic connection within a body of experimental and independent (or non-academic) sound-based artworks produced in the past few decades. As it was argued, although realised in different mediums and belonging to different artistic categories, such works are connected through a certain aesthetic sensibility. Nevertheless, since the majority of these works have thus far received little scholarly attention prior to this research, a framing discussion of the aesthetic principles and features that link them seemed overdue. With this in mind, the last section of this research examined this emergent phenomenon, scrutinising the particular aesthetic features that connect such soundbased artworks, arguing for a more specific terminology to adequately account for this aesthetic across the various practices in which it is observed. Rejecting "minimalist" as a descriptor, this chapter called for an aesthetic frame of reference derived through Brutalism, understood as a crystallisation of key features of modernism and its various movements. Subsequently, The Brutalist Noise Ensemble and machine brut(e) were presented as conscious efforts to create sound art redolent of Brutalism, locating this research in the context of the revival of Brutalism in recent years, which, as was argued, can be expanded to works from a wide range of contemporary artists and musicians. In this way, aside from its effort to draw more attention to an emergent sense of aesthetic among sound-based works of art, this thesis also aimed to prompt the engagement of sound artists in this neo-brutalism.

\subsection{Future Work}

Due to the truly multidisciplinary nature of this research, there are a number of different paths in which it can continue and evolve. Experimenting with alternative parts (such as different kinds of DC motors or metal sheets) and growing the number of instrumenttypes to expand the frequency and timbral ranges of the ensemble are obvious possibilities. In particular, developing instrument-types whose sonic output lie in the low 
frequency range (below 200Hz) would notably expand the sonic palette of the ensemble. However, due to the physics of audible low frequency vibrations, and considering the fact that this research focuses on mechanically generated sound, this would require considerably larger parts, and consequently, larger sound-sculptures.

Remodelling the design for larger-scale sound-sculptures would be another way to expand the work carried out in this research. As argued throughout the thesis, the physicality and audiovisual expressivity of the work has been one of the key considerations in the development of this research. Accordingly, enlarging the scale of the ensemble and using more sizable sound-sculptures further facilitates the audiovisual connectivity of the work. This can be particularly helpful in performance settings, where the distance between the sound-sculptures and the audience could be a hindrance in their visual perception of the sound-generating units.

Larger sound-sculptures would naturally provide higher volume levels, which can also benefit some performance scenarios. Since the physicality of the sound-generating process achieved through an entirely mechanical apparatus is an essential principle by which the work is created, amplification has always been avoided during performances. This can potentially cause problems in larger spaces and big venues, and lead to rather quiet performances. Larger instruments with larger sound-producing parts would equip the performance with higher maximum loudness. Another way to achieve this would of course be expanding the number of each instrument-type in the ensemble. This can make feasible not only a louder performance setting when required, but also installation settings in which a large number of sound-sculptures surround the audience and provide an immersive audiovisual experience.

Lastly, working towards collaborative projects in the creation of large-scale sonic artworks with architectural qualities would be an anticipative pathway to further explore the interconnections between this research and Brutalist architecture, and to solidify the concept of sound-based brutalism coined in this thesis. 


\subsection{Novel Contributions}

This section summarises the key novel contributions of this research, and lists its peerreviewed academic and artistic outputs and achievements that support the validity and significance of these contributions to the academic and art communities.

\subsubsection{Primary Contributions}

1. Formalising a framework for aesthetic enhancement of extra-musical sounds through the use of metric and grid-based rhythms and highlighting the visual aspect of the sound-production.

2. Establishing a new categorisation with regard to the employment of mechatronics in music and sound art by organising the body of works into 'musical robotics' and 'mechatronic sound-objects'.

3. Design and development of an ensemble of new mechatronic sound-sculptures comprising three novel instruments:

- Rasper

- Ripper

- Mutor

4. Creating three original artworks combining mechatronic sound art and minimal music, urban noise, and cellular automata.

5. Formalising a new aesthetic platform in the study of contemporary sound-based works of art which received little to no academic attention prior to this research.

6. Expanding the territory of glitch music into the realm of physically generated sound.

7. Coining "sound-based brutalism", and extending the revival of Brutalism from architecture and visual arts into sonic arts, by the creation of Brutalist-inspired soundbased artworks.

\subsubsection{Research Outputs}

This section presents the list of awards, peer-reviewed journal and conference proceedings publications, performances and installation works, and media features and interviews that have arisen from this research. 


\subsubsection{Awards}

Winner of $1^{\text {st }}$ Prize in Sonic Arts Award 2015 for Sound Art category.

Winner of $3^{\text {rd }}$ Prize in the NZSM Composers Competition 2014.

\subsubsection{Refereed Publications}

1. Mo H. Zareei, Ajay Kapur, Dale A. Carnegie. "Noise on the Gird: Rhythmic Pulse in Experimental and Electronic Noise Music," In Proceedings of the 2013 International Computer Music Conference (ICMC), Perth, Australia (2013).

2. Mo H. Zareei, Dugal McKinnon, Ajay Kapur, and Dale A. Carnegie. "complex: Physical Re-sonification of Urban Noise," In Proceedings of the 2014 International Conference on Auditory Display (ICAD), New York, NY (2014).

3. Mo H. Zareei, Ajay Kapur, Dale A. Carnegie. "Rasper: a Mechatronic Noise-intoner," In Proceedings of the 2014 International Conference on New Interfaces for Musical Expression (NIME), London, UK (2014).

4. Mo H. Zareei, Dale A. Carnegie, Ajay Kapur, and Dugal McKinnon. "Mutor. Drone Chorus of Metrically Muted Motors," In Proceedings of the 2014 International Computer Music Conference-Sound and Music Computing (ICMC-SMC), Athens, Greece (2014).

5. Mo H. Zareei, Dale A. Carnegie, Ajay Kapur. "NOISE SQUARE: Physical Sonification of Cellular Automata through Mechatronic Sound-sculpture," In Proceedings of the 2015 International Symposium on Electronic Art (ISEA), Vancouver, Canada (2015).

6. Mo H. Zareei, Dale A. Carnegie, Ajay Kapur, and Dugal McKinnon. "Rippler: a Mechatronic Sound-sculpture,” Journal of Comparative Media Arts 1 (2015).

7. Mo H. Zareei, Ajay Kapur, Dale A. Carnegie. "Rasping Music: Remodelling Early Minimalist Music through Mechatronic Sound-sculpture," In Proceedings of the 2015 International Computer Music Conference (ICMC), Denton, TX (2015).

8. Mo H. Zareei, Dale A. Carnegie, Ajay Kapur. "Physical Glitch Music: A Brutalist Noise Ensemble," Leonardo Music Journal 25 (2015).

9. Mo H. Zareei, Dugal McKinnon, Dale A. Carnegie, and Ajay Kapur. "Sound-based Brutalism: an Emergent Aesthetic," Organised Sound 21(1) (Forthcoming 2016).

\subsubsection{Performances \& Installations}

Brazen Bull Love. Live performance at the International Computer Music Conference (ICMC). August, 2013. The Bakery, Perth, Australia. 
Rasping Music. Audiovisual installation at Sonic Arts and Engineering Expo. October 2013. Victoria University, Wellington, New Zealand.

Rasping Music. Live performance at Wound Sound. November 2013. City Gallery Wellington, Wellington, New Zealand.

complex. Audiovisual installation at the International Conference on Auditory Display (ICAD). June 2014. NYU Magnet, New York, US.

Abrasive Stanza No. 1. Live performance at the International Conference on New Interfaces for Musical Expression (NIME). June 2014. Deptford Town hall, London, UK.

Rasping Music. Audiovisual installation at Lux festival. August 2014. Eva Street (public artwork), Wellington, New Zealand.

Rasping Music. Live performance at Composers Competition. September 2014. Adam Concert Room, Wellington, New Zealand.

Abrasive Stanza No. 1. Live performance at Tehran Annual Digital Arts Expo (TADAEX). October 2014. Gholhak Cinemateque, Tehran, Iran.

Artifice. Live performance at the International Symposium on Electronic Art (ISEA). November 2014. The Jamjar Gallery, Dubai, UAE.

Gradient. Audiovisual installation at Low Noise Vol. 2. February 2015. Toi Poneke Gallery, Wellington, New Zealand.

The Brutalist Noise Ensemble. Live performance at Low Noise Vol. 2. February 2015. Toi Poneke Gallery, Wellington, New Zealand.

machine brut(e). Series of ten installation pieces. May 2015. Adam Art Gallery. Wellington, New Zealand.

NOISE SQUARE. Audiovisual installation at the International Symposium on Electronic Art (ISEA). August 2015. Vancouver Art Gallery, Vancouver, Canada.

\subsubsection{Media, Press}

"Brutalist Architecture informs a series of sound-sculptures", Featured on The Creators Project. Available at: http://thecreatorsproject.vice.com/blog/brutalist-architecturesound-sculptures.

"Artist Gives Brutalist Architecture a Singing Voice", Featured on Kill Screen. Available at: https://killscreen.com/articles/hard-love-brute/. 
"Robotics and Music of the Future", Interview with Radio New Zealand. Available at: http://www.radionz.co.nz/national/programmes/standing-roomonly/audio/20157315/robotics-and-music-of-the-future.

"Mo H. Zareei: gradient", Interview with SOUNZ, centre for New Zealand Music. Available at: http://sounz.org.nz/resources/show/1264.

"The Story and Kinetic Sound-sculptures of Mo Zareei", Profiled on Streaming Museum. Available at: http://streamingmuseum.org/mh-zareei.

"Mechatronic Sound-sculptures by Mo H. Zareei", Featured on Creative Applications. Available at: http:/ / www.creativeapplications.net/sound/rasper-mutor-and-rippler.

"Sound-sculptures Put on an Electrifying Musical Performance", Featured on Atmel blog. Available at: http://blog.atmel.com/2015/05/19/sound-sculptures-put-on-anelectrifying-steel-drum-performance.

"These Brutalist-inspired Instruments Redefine the Meaning of Industrial Music", Featured on Fast Company Design. Available at:

http://www.fastcodesign.com/3046482/these-brutalist-inspired-instrumentsredefine-the-meaning-of-industrial-music. 


\subsection{Denouement}

You are sitting in a cafe on a Tuesday afternoon. In the background a pop record is playing. Suddenly the CD stutters. You have listened to glitch music for quite a while now. Your reaction has changed. Instead of the usual frustrated response you lean back and enjoy the random loops and skips of the CD, finding it more beautiful in its simplicity than the commercial hit from which it derives. You hear how well it goes with the cappuccino-maker's noise, the cell phone ringing at another table and the chiming from tablespoons on teacups and of forks on plates [25].

Fascinated by the post-Industrial Revolution soundscapes, the Italian Futurist believed that "every manifestation of life is accompanied by noise" [2]. Thus, his Futurist manifesto, in addition to a call for expanding the realm of musical sound, was indeed an effort towards embodiment of the modern industrial life in music. Interestingly enough, as Russolo invites the post-Industrial Revolution city-dwellers to "orchestrate together in [their] imagination the din of rolling shop shutters, ... electrical plants and subways" [2], the Brutalist mind, at the other front, "tries to face up to a mass-production society, and drag a rough poetry out of the confused and powerful forces which are at work" [108]. With this in mind, while "the experience of everyday life is increasingly mediated by a multitude of mechanically reproduced sounds" [124], the research work undertaken for this thesis is an effort to embrace the potential aesthetics of the noisy machines surrounding urban technological life. In doing so, it adheres to a brutalist line of thought through appreciation of the very physical existence of its conventionally "anti-beauty" raw material, by expressing them in clear visual and sonic structures.

The characteristic of noise is that of reminding us brutally of life.

- Luigi Russolo 
Parts of this chapter were featured in the following publication:

Mo H. Zareei, Dale A. Carnegie, Ajay Kapur. "Physical Glitch Music: A Brutalist Noise Ensemble," Leonardo Music Journal 25. 


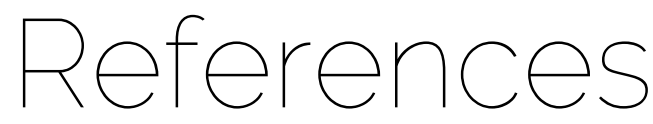

[1] H. Cowell, "The Joys of Noise," in Audio Culture: Readings in Modern Music, C. Cox and D. Warner, Eds. New York: Continuum, 2004.

[2] L. Russolo, The Art of Noises. New York: Pendragon Press, 1986.

[3] J. Cage, "The Future of Music: Credo," in Audio Culture: Readings in Modern Music, C. Cox and D. Warner, Eds. New York: Continuum, 2004.

[4] H. L. Helmholtz, On the Sensations of Tone as a Physiological Basis for the Theory of Music. Cambridge University Press, 2009.

[5] T. Wishart, On sonic art. Psychology Press, 1996.

[6] S. Voegelin, Listening to Noise and Silence: Towards a Pbilosophy of Sound Art. Bloomsbury Publishing USA, 2010.

[7] E. Pouncey, "Consumed by Noise," The Wire, vol. 198, pp. 26-32, 2000.

[8] E. Varèse and C. Wen-chung, "The Liberation of Sound," Perspect. New Music, vol. 5, no. 1, pp. 11-19, 1966.

[9] R. M. Schafer, The Book of Noise. Arcana Editions, 1998.

[10] C. E. Shannon and W. Weaver, The Mathematical Theory of Communication, vol. 19. University of Illinois Press, 1949.

[11] M. Serres, Genesis. Ann Arbor: University of Michigan Press, 1995.

[12] C. Taylor, "Noise is OK," Semiotica, vol. 52, no. 3-4, pp. 273-289, 1984.

[13] D. Kahn, Noise, Water, Meat: a History of Sound in the Arts. Cambridge, Mass.: MIT press, 1999.

[14] P. Boulez, Boulez on Music Today. Cambridge, Mass.: Harvard University Press, 1971.

[15] A. Evens, Sound Ideas: Music, Machines, and Experience. Minneapolis, MN: University of Minnesota Press, 2005.

[16] M. Schedel, "Anticipating Interactivity: Henry Cowell and the Rhythmicon," Organised Sound, vol. 7, no. 3, pp. 247-254, 2002.

[17] J. Attali, Noise: the Political Economy of Music. Manchester University Press, 1985.

[18] L. Landy, Understanding the Art of Sound Organization. Cambridge, Mass.: MIT Press, 2007.

[19] K. Cascone, "The Aesthetics of Failure: 'Post-digital' Tendencies in Contemporary Computer Music,” Comput. Music J., vol. 24, no. 4, pp. 12-18, 2000.

[20] A. Collis, "Sounds of the System: the Emancipation of Noise in the Music of Carsten Nicolai," Organised Sound, vol. 13, no. 1, p. 31, 2008.

[21] C. Kelly, Cracked Media: the Sound of Malfunction. Cambridge, Mass.: The MIT Press, 2009.

[22] P. Hegarty, Noise/Music: a History. Bloomsbury Academic, 2007. 
[23] G. Hainge, "Of Glitch and Men: the Place of the Human in the Successful Integration of Failure and Noise in the Digital Realm," Commun. Theory, vol. 17, no. 1, pp. 26-42, 2007.

[24] J. T. Demers, Listening through the Noise: the Aesthetics of Experimental Electronic Music. New York: Oxford University Press, 2010.

[25] T. Sangild, "Glitch-The beauty of malfunction," in Bad Music: the Music We Love to Hate, C. Washburne and M. Derno, Eds. New York, NY: Routledge, 2004, pp. 257-74.

[26] P. Sherburne, “12k: Between Two Points,” Organised Sound, vol. 6, no. 3, pp. 171176, 2001.

[27] T. Sangild, Glitch_The beauty of malfunction. New York: Routledge, 2004.

[28] B. Neilll, "Pleasure Beats: Rhythm and the Aesthetics of Current Electronic Music," Leonardo Music J., vol. 12, pp. 3-6, 2002.

[29] J. Chadabe, "Remarks on Computer Music Culture," Comput. Music J., vol. 24, no. 4, pp. 9-11, 2000.

[30] C. B. Fowler, "The Museum of Music: A History of Mechanical Instruments," Music Educ. J., vol. 54, no. 2, pp. 45-49, 1967.

[31] J. Murphy, A. Kapur, and D. Carnegie, "Musical Robotics in a Loudspeaker World: Developments in Alternative Approaches to Localization and Spatialization," Leonardo Music J., vol. 22, no. 1, pp. 41-48, 2012.

[32] E. Singer, J. Feddersen, C. Redmon, and B. Bowen, "LEMUR's Musical Robots," in Proceedings of the 2004 International Conference on New Interfaces for Musical Expression (NIME), 2004, pp. 181-184.

[33] E. Singer, K. Larke, and D. Bianciardi, "LEMUR GuitarBot: MIDI Robotic String Instrument," in Proceedings of the 2003 International Conference on New Interfaces for Musical Expression (NIME), 2003, pp. 188-191.

[34] G. Weinberg and S. Driscoll, "Toward Robotic Musicianship," Comput. Music J., vol. 30, no. 4, pp. 28-45, 2006.

[35] A. Kapur, M. Darling, D. Diakopoulos, J. W. Murphy, J. Hochenbaum, O. Vallis, and C. Bahn, "The Machine Orchestra: an Ensemble of Human Laptop Performers and Robotic Musical Instruments," Comput. Music J., vol. 35, no. 4, pp. 49-63, 2011.

[36] J. Murphy, J. McVay, A. Kapur, and D. Carnegie, "Designing and Building Expressive Robotic Guitars," in Proceedings of the 2013 International Conference on New Interfaces for Musical Expression (NIME), 2013.

[37] A. Kapur, "A History of Robotic Musical Instruments," in Proceedings of the 2005 International Computer Music Conference (ICMC), 2005, pp. 21-28.

[38] A. Kapur, J. Murphy, and D. Carnegie, "Kritaanjali: A Robotic Harmonium for Performance, Pedogogy and Research," in Proceedings of the 2012 International Conference on New interfaces for Musical Expression (NIME), 2012.

[39] G.-W. Raes, “A Personal Story of Music and Technologies," Leonardo Music J., vol. 2, no. 1, pp. 29-35, 1992.

[40] A. Focke, Trimpin: Contraptions for Art and Sound. University of Washington Press, 2011. 
[41] J. Strouse, "Music of the Spheres," in Trimpin: contraptions for art and sound, A. Focke, Ed. University of Washington Press, 2011.

[42] G. Monahan, "Multiple Machine Matrix (Sound Installation)," 1994. [Online]. Available: www.gordonmonahan.com. [Accessed: 03-Oct-2013].

[43] P. Garland, "Gordon Monahan: Machines and the Sounds That Give Them Life," www.gordonmonaban.com, 1993. .

[44] A. Licht, Sound Art: Beyond Music, Between Categories. Rizzoli International Publications, 2007.

[45] "La chambre de machines," http:/ / wmw.lachambredesmachines.com/. .

[46] J. Cowley, "Nicolas Bernier and Martin Messier: Object-based electronic music," Music Works, no. 115, 2013.

[47] N. Bernier, "Frequencies (a)," Nicolas Bernier, 2012. [Online]. Available: www.nicolasbernier.com. [Accessed: 02-Sep-2014].

[48] E. Rose, "Translating Transformations: Object-Based Sound Installations," Leonardo Music J., vol. 23, pp. 65-69, 2013.

[49] J. Murphy and A. Kapur, "The Gallery as an Instrument: Using Remote Sensing Technology to Interface with Musical Robotics," in Proceedings of the 2011 Australian Computer Music Conference (ACMC), 2011.

[50] "This Orchestra is Made from Lamps and Electricity," Wired UK. [Online]. Available: http://www.wired.co.uk/news/archive/2014-12/22/trippy-orchestra. [Accessed: 23-Mar-2015].

[51] D. Diakopoulos and A. Kapur, "HIDUINO: A Firmware for Building Driverless USB-MIDI Devices Using the Arduino Microcontroller," in Proceedings of the 2011 International Conference on New Interfaces for Musical Expression (NIME), 2011.

[52] "About the Hand Crafted Instruments," New Music Co-op. [Online]. Available: http://www.newmusiccoop.org/past/russolo/about_instruments.php. [Accessed: 30-Dec-2014].

[53] H. Fletcher and W. A. Munson, "Loudness, Its Definition, Measurement and Calculation," Bell Syst. Tech. J., vol. 12, no. 4, pp. 377-430, 1933.

[54] M. Mauermann, G. R. Long, and B. Kollmeier, "Fine Structure of Hearing Threshold and Loudness Perception," J. Acoust. Soc. Am., vol. 116, no. 2, pp. 1066-1080, Aug. 2004.

[55] E. Strickland, Minimalism: Origins. Indiana University Press, 1993.

[56] K. Baker, Minimalism: Art of Circumstance. Abbeville Press, 1988.

[57] K. Potter, Four Musical Minimalists: La Monte Young, Terry Riley, Steve Reich, Philip Glass. Cambridge University Press, 2002.

[58] T. Johnson, The Voice of New Music: New York City, 1972-1982 - A Collection of Articles Originally Published in the Village Voice by Tom Johnson. Eindhoven, Netherlands: Het Apollohuis, 1989.

[59] M. Nyman, Experimental Music: Cage and Beyond. Cambridge University Press, 1999.

[60] M. Nyman, H. Davis, and R. Orton, "Steve Reich: an Interview with Michael Nyman," The Musical Times, no. 112/1537, pp. 229-231, 1971.

[61] S. Reich, Steve Reich: Writings About Music. Halifax, NY: Art Metropole, 1974.

[62] R. Alsop, "Exploring the Self Through Algorithmic Composition," Leonardo Music J., vol. 9, pp. 89-94, Dec. 1999. 
[63] B. L. Jacob, "Algorithmic Composition as a Model of Creativity," Organised Sound, vol. 1, no. 03, pp. 157-165, 1996.

[64] G. Nierhaus, Algorithmic Composition: Paradigms of Automated Music Generation. Springer Science and Business Media, 2009.

[65] A. Dorin, "Generative Processes and the Electronic Arts," Organised Sound, vol. 6, no. 1, pp. 47-53, 2001.

[66] A. W. Burks, Essays on Cellular Automata. University of Illinois Press, 1970.

[67] E. R. Miranda, "Cellular Automata Music: From Sound Synthesis to Musical Forms," in Evolutionary Computer Music, E. R. Miranda and J. A. Biles, Eds. London: Springer, 2007, pp. 170-193.

[68] S. Wolfram, "Universality and Complexity in Cellular Automata," Phys. Nonlinear Phenom., vol. 10, no. 1, pp. 1-35, 1984.

[69] D. Millen, "Cellular Automata Music," in Proceedings of the 1990 International Computer Music Conference (ICMC), 1990, pp. 314-316.

[70] B. A. Varga, Conversations with Iannis Xenakis. Faber \& Faber, 1996.

[71] D. Burraston and E. Edmonds, "Cellular Automata in generative electronic music and sonic art: a historical and technical review.," Digit. Creat., vol. 16, no. 3, 2005.

[72] E. R. Miranda, "Chaosynth: Computer Music Meets High-Performance Computing," Supercomputer, vol. 11, no. 1, pp. 16-23, 1994.

[73] D. Burraston, E. Edmonds, D. Livingstone, and E. R. Miranda, "Cellular Automata in MIDI-Based Computer Music," in Proceedings of 2004 the International Computer Music Conference (ICMC), 2004, pp. 71-78.

[74] P. Beyls, "The Musical Universe of Cellular Automata," in Proceedings of the 1989 International Computer Music Conference (ICMC), Columbus, Ohio, 1989.

[75] P. Beyls, "Musical Morphologies from Self-organizing Systems," J. New Music Res., vol. 19, no. 2-3, pp. 205-218, 1990.

[76] P. Beyls, "Cellular Automata Mapping Procedures," in Proceedings of the 2004 International Computer Music Conference (ICMC), 2004, pp. 55-58.

[77] M. Gardner, "Mathematical Games: the Fantastic Combinations of John Conway's New Solitaire Game 'life,"' Sci. Am., vol. 223, no. 4, pp. 120-123, 1970.

[78] "Evil/Live," Bill Vorn, $1997 . \quad$ [Online]. Available: http://billvorn.concordia.ca/robography/Evil3.html.

[79] A. Dorin, "LiquiPrism: Generating polyrhythms with cellular automata," in Proceedings of the 2002 International Conference on Auditory Display, Kyoto, Japan, July 2, 2002, vol. 5.

[80] J. He and A. Kapur, "Formalization Using Organic Systemization in Musical Applications," in Proceedings of the 2013 International Conference on Computation, Communication, Aesthetics and X (xCo Ax), 2013.

[81] A. Eigenfeldt, "Artworks," Arne Eigenfeldt personal webpage. [Online]. Available: https://aeigenfeldt.wordpress.com/works. [Accessed: 10-Dec-2014].

[82] J. A. Fisher, "What the Hills Are Alive with: in Defense of the Sounds of Nature," J. Aesthet. Art Crit., vol. 56, no. 2, pp. 167-179, 1998.

[83] M. Raimbault and D. Dubois, "Urban Soundscapes: Experiences and Knowledge," Cities, vol. 22, no. 5, pp. 339-350, 2005. 
[84] R. M. Schafer, The New Soundscape: a Handbook for the Modern Music Teacher. BMI Canada, 1969.

[85] A. Y. Kelman, "Rethinking the Soundscape: a Critical Genealogy of a Key Term in Sound Studies," Senses Soc., vol. 5, no. 2, pp. 212-234, 2010.

[86] R. M. Schafer, Our Sonic Environment and the Soundscape: the Tuning of the World. Inner Traditions / Bear \& Co, 1993.

[87] E. A. Thompson, The Soundscape of Modernity: Architectural Acoustics and the Culture of Listening in America, 1900-1933. MIT Press, 2004.

[88] W. C. Williams, "George Antheil and the Cantilene Critics: a Note on the First Performance of Antheil's Music in New York City, April 10, 1927," Transition., no. 13, 1928.

[89] P. Rosenfeld, An Hour with American Music. Hyperion Press, 1929.

[90] T. H. Park, J. Turner, C. Jacoby, A. Marse, M. Musick, A. Kapur, and J. He, "Locative Sonification: Playing the World through Citygram," presented at the Proceedings of the 2013 International Computer Music Conference (ICMC), Perth, Australia, 2013.

[91] P. Thomson, "Atoms and Errors: Towards a History and Aesthetics of Microsound," Organised Sound, vol. 9, no. 2, pp. 207-218, 2004.

[92] T. Myatt, "New Aesthetics and Practice in Experimental Electronic Music," Organised Sound, vol. 13, no. 1, pp. 1-3, 2008.

[93] T. Myatt, P. Worth, M. Fell, and T. Blake, "New Aesthetics in Computer Music," 2010. [Online]. Available: http://www.york.ac.uk/music/mrc/projects/nacm/. [Accessed: 02-Feb-2015].

[94] P. Sherburne, "Digital Discipline: Minimalism in House and Techno," in Audio Culture: Readings in Modern Music, New York, NY: Continuum, 2008, pp. 319-326.

[95] A. Szepanski, "A Mille Plateaux Manifesto," Organised Sound, vol. 6, no. 3, p. 2001.

[96] B. Borthwick, "The Perfect Strom," The Wire, vol. 238 (December), pp. 40-7, 2003.

[97] O. Bender, "Interview with Olaf Bender A.K.A Byetone," 2008.

[98] S. Hofer, “'Atomic' Music: Navigating Experimental Electronica and Sound Art through Microsound," Organised Sound, vol. 19, no. 3, pp. 295-303, 2014.

[99] F. Colpitt, Minimal Art: The Critical Perspective. University of Washington Press, 1993.

[100] M. P. Wright, “Zimoun," EAR ROOM. [Online]. Available: https://earroom.wordpress.com/2011/01/01/zimoun/. [Accessed: 06-Mar2015].

[101] K. Gann, "Thankless Attempts at a Definition of Minimalism," in Audio Culture: Readings in Modern Music, New York: Continuum, 2008, pp. 299-303.

[102] W. L. Ashline, "Clicky Aesthetics: Deleuze, Headphonics, and the Minimalist Assemblage of Aberrations,"' Strateg. J. Theory Cult. Polit., vol. 15, no. 1, pp. 87$101,2002$.

[103] J. D. Knowles, “Alva Noto,” Filter, vol. 62, pp. 17-19, 2006.

[104] "Interview with the artist Nicolas Bernier — LABoral Centro de Arte y Creación Industrial," LABoral Cento De Arte, 2014. [Online]. Available: 
http:/ /www.laboralcentrodearte.org/en/files/2013/bloglaboral/entrevista-alartista-canadiense-nicolas-bernier/view. [Accessed: 09-Apr-2015].

[105] A. Vidler, "Brutalism: Aesthetic or Ethic?," CLOG: Brutalism, pp. 16-17, 2013.

[106] R. Banham, The New Brutalism: Ethic or Aesthetic?. London, UK: Architectural Press, 1966.

[107] R. Banham, “The New Brutalism," October, vol. 136, pp. 19-28 (originally in Architectural Review, December 1955, pp. 354-61.), Apr. 2011.

[108] A. Smithson and P. Smithson, "The New Brutalism," October, vol. 136, p. 37 (originally in Architectural Design 27, April 1957, p. 113), Apr. 2011.

[109] M. J. Lewis, “The 'new' New Brutalism," New Criterion, vol. 33, p. 19, Dec-2014.

[110] J. Macarthur, "The Nomenclature of Style: Brutalism, Minimalism, Art History and Visual Style in Architecture Journals," Archit. Theory Rev., vol. 10, no. 2, pp. 100-108, 2005.

[111] J. Reidel, "Ugly," CLOG: Brutalism, pp. 126-127, 2013.

[112] J. Glancey, "Brutalism: How unpopular buildings came back in fashion," BBC Culture, 2014. [Online]. Available: http://www.bbc.com/culture/story/20140828why-brutal-is-beautiful. [Accessed: 13-Mar-2015].

[113] G. F. Shapiro, "Brutalism and Landscape Desire," CLOG: Brutalism, pp. 100-101, 2013.

[114] F. Gallanti, "Sigurd Lewerentz Enigmatic Brutalism," CLOG: Brutalism, pp. 82-83, 2013.

[115] W. Villacorta and C. Marsollier, "Candidate," CLOG: Brutalism, pp. 156-157, 2013.

[116] S. Hicks and A. Newmeyer, "Save the Prentice Wrecking Ball: The Monument to Bruce," CLOG: Brutalism, pp. 158-159, 2013.

[117] R. Berger, "Coliseum Memorial," CLOG: Brutalism, pp. 160-161, 2013.

[118] B. Stark, "Brutalism in the Mountains," CLOG: Brutalism, pp. 96-97, 2013.

[119] S. Sterken, "Music as an Art of Space: Intersections Between Music and Architecture in the Work of Iannis Xenakis," in Resonance: Essays on the Intersection of Music and Architecture, Ames, IA: Culicidae Architectural Press, 2007, pp. 21-51.

[120] P. Hegarty, "Just What is it that Makes Today's Noise Music so Different, so Appealing?," Organised Sound, vol. 13, no. 1, pp. 13-20, 2008.

[121] "Bunkers, Brutalism and Bloodymindedness: Concrete Poetry with Jonathan Meades," BBC, BBC FOUR, 2014.

[122] A. Clement, Brutalism: Post-War British Architecture. Ramsbury: Crowood Press, 2011.

[123] E. Bates, "Glitches, Bugs, and Hisses: The Degeneration of Musical Recordings and the Contemporary Musical Work," in Bad Music: the Music we Love to Hate, New York, NY: Routledge, 2004.

[124] M. Bull and L. Back, "Introduction: Into Sound," in The Auditory Culture Reader, Berg Oxford, 2003.

[125] D. Shiffman, S. Fry, and Z. Marsh, The Nature of Code. D. Shiffman, 2012. 


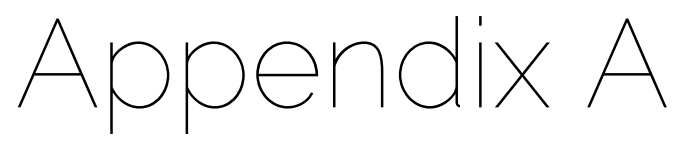

\section{The Driver Board (code)}

The following Arduino code is developed and uploaded to the Arduino Mega in order to drive the instruments. Each component (that is actuator or LED) is addressed through a MIDI pitch number, and controlled by the MIDI velocity value.

Two Adafriut 16-Channel 12-bit PWM/Servo Driver boards are combined with the Arduino in order to provide up to 32 PWM outputs when needed (such as running the full ensemble).

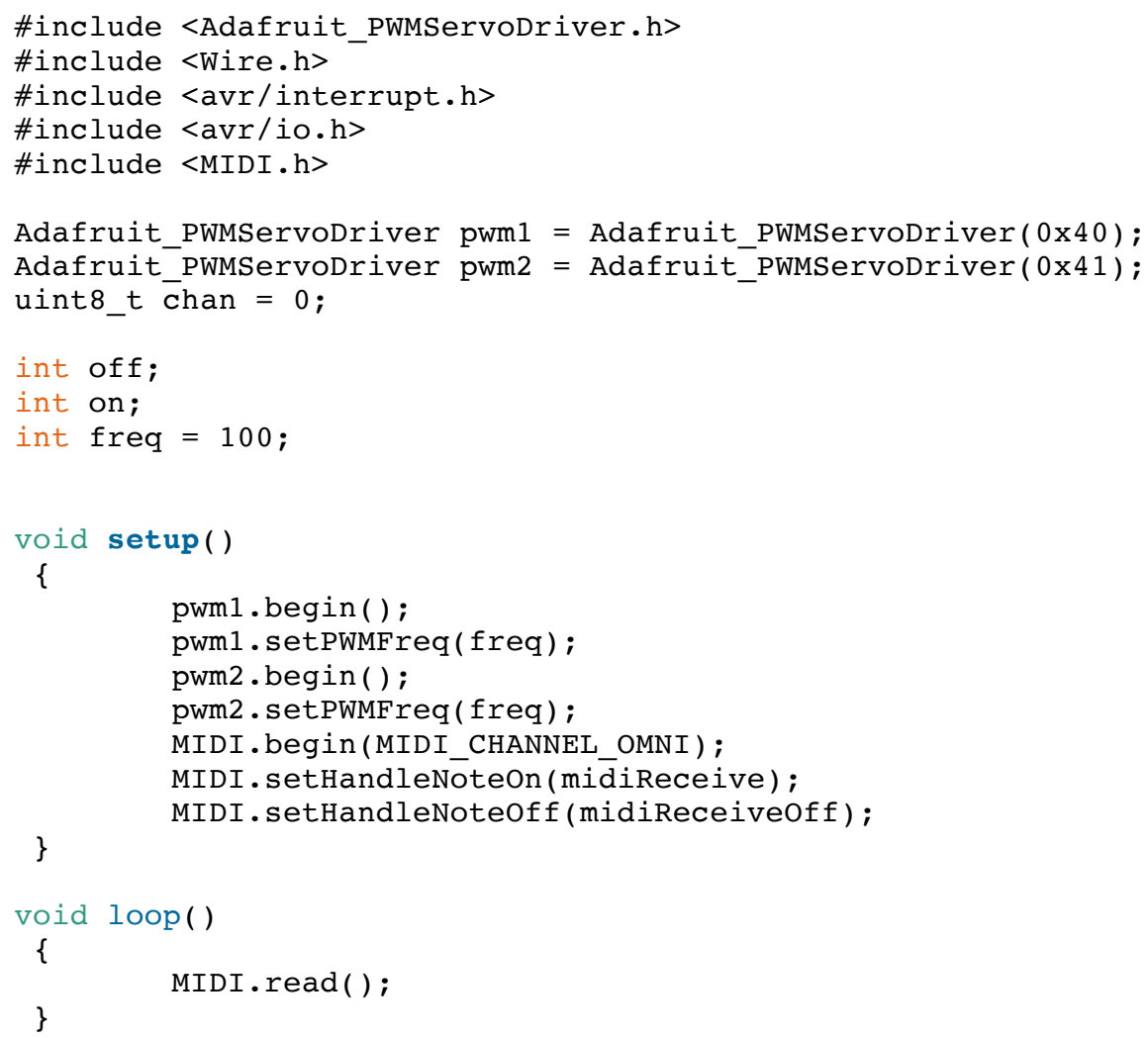




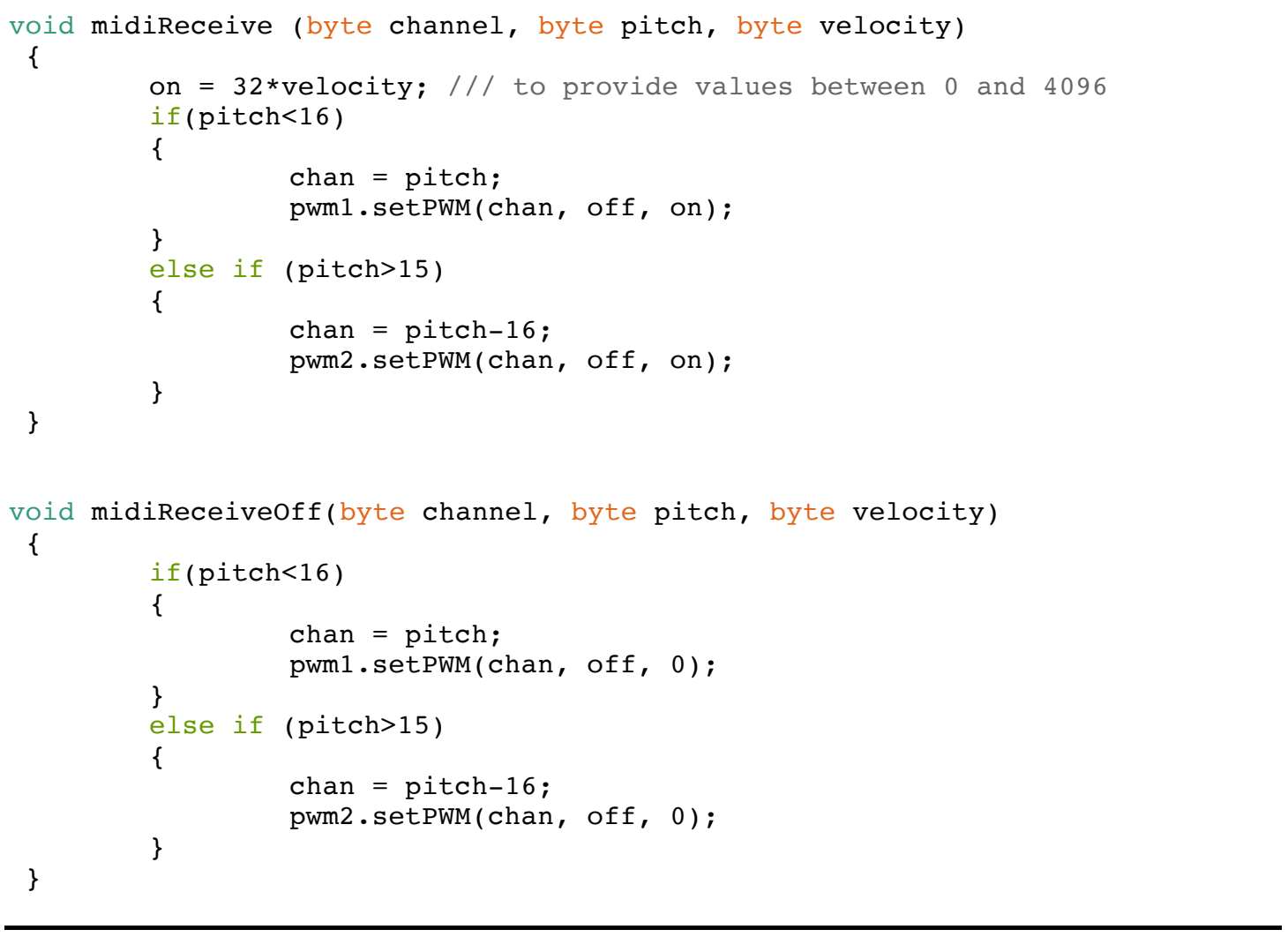




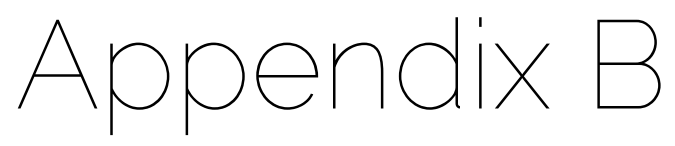

This section provides supplemental technical details with regard to the compositions discussed in this thesis. This includes the codes used for complex and NOISE SQUARE, and the score used for a live performance rendition of Rasping Music.

\section{Rasping Music (score)}

A performance realisation of Rasping Music was arranged for four Raspers and laptopproduced electronics for the Composers Competition 2014 concert at New Zealand School of Music's Adam Concert Room. Following a diagram of the signal flow and the score legend, this section presents the score used for this performance.

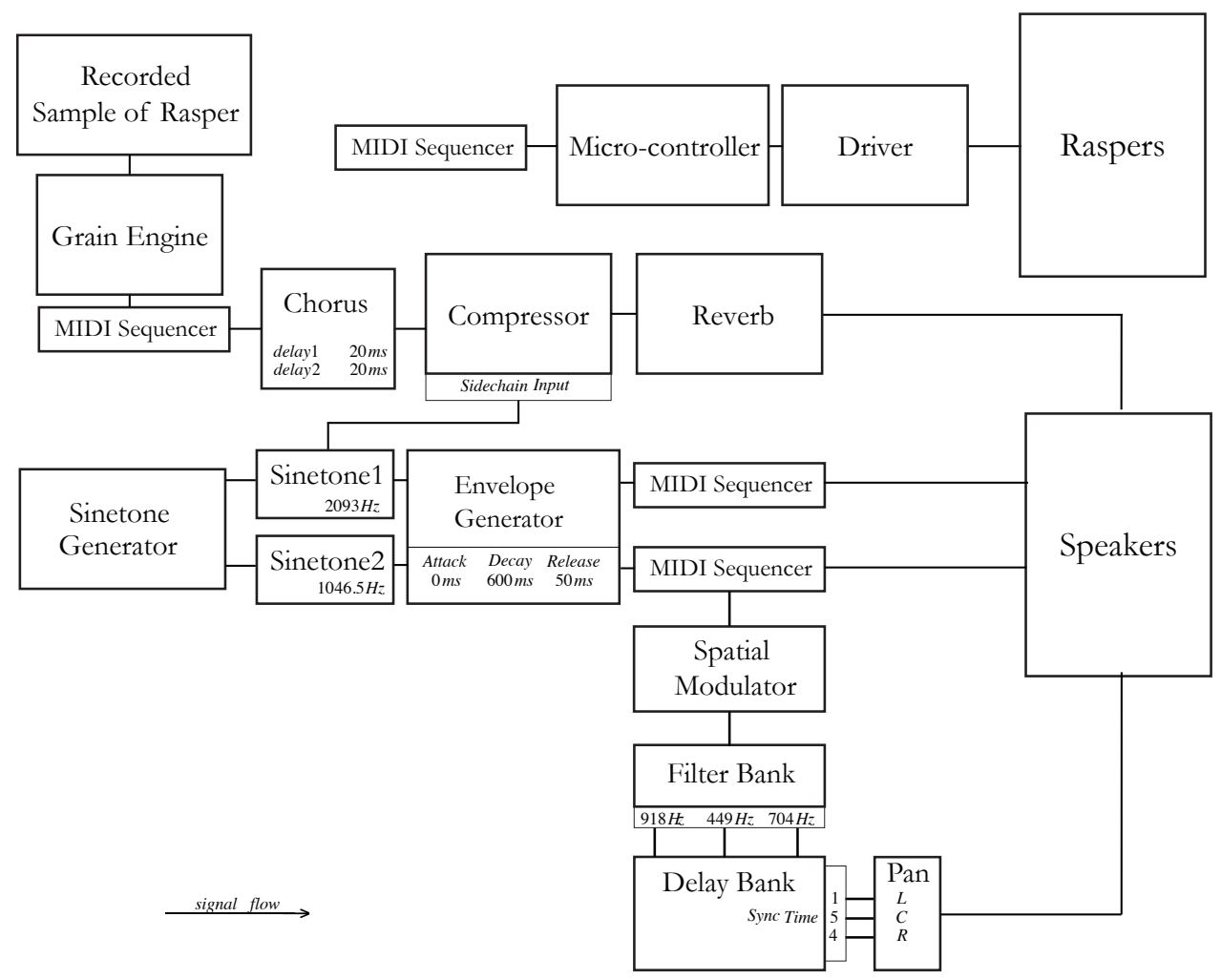




\section{silence. \\ fade in.}

fade out.

$\overrightarrow{. . .}$ or $\ldots$ continue fade.

I $0 \mathrm{db}$.

$f x \mapsto \quad$ filter delay effects chain on.

$\therefore$ to ......... delay feedback, min to max.

$\therefore \quad$ spatial modulation effect, dry and wet.

s

$\underset{\rightarrow}{40 \mathrm{~Hz}}$ modulation frequency, gradually reach $40 \mathrm{~Hz}$.

$\vec{c}-\Phi] \not \subset$ is sidechained by the signal from $c$.

$f x \mid$ filter delay effects chain off.

: $\rangle$ turn chorus feedback on.

$\langle:|$ turn chorus feedback off.

::*:::: reverberated signal.

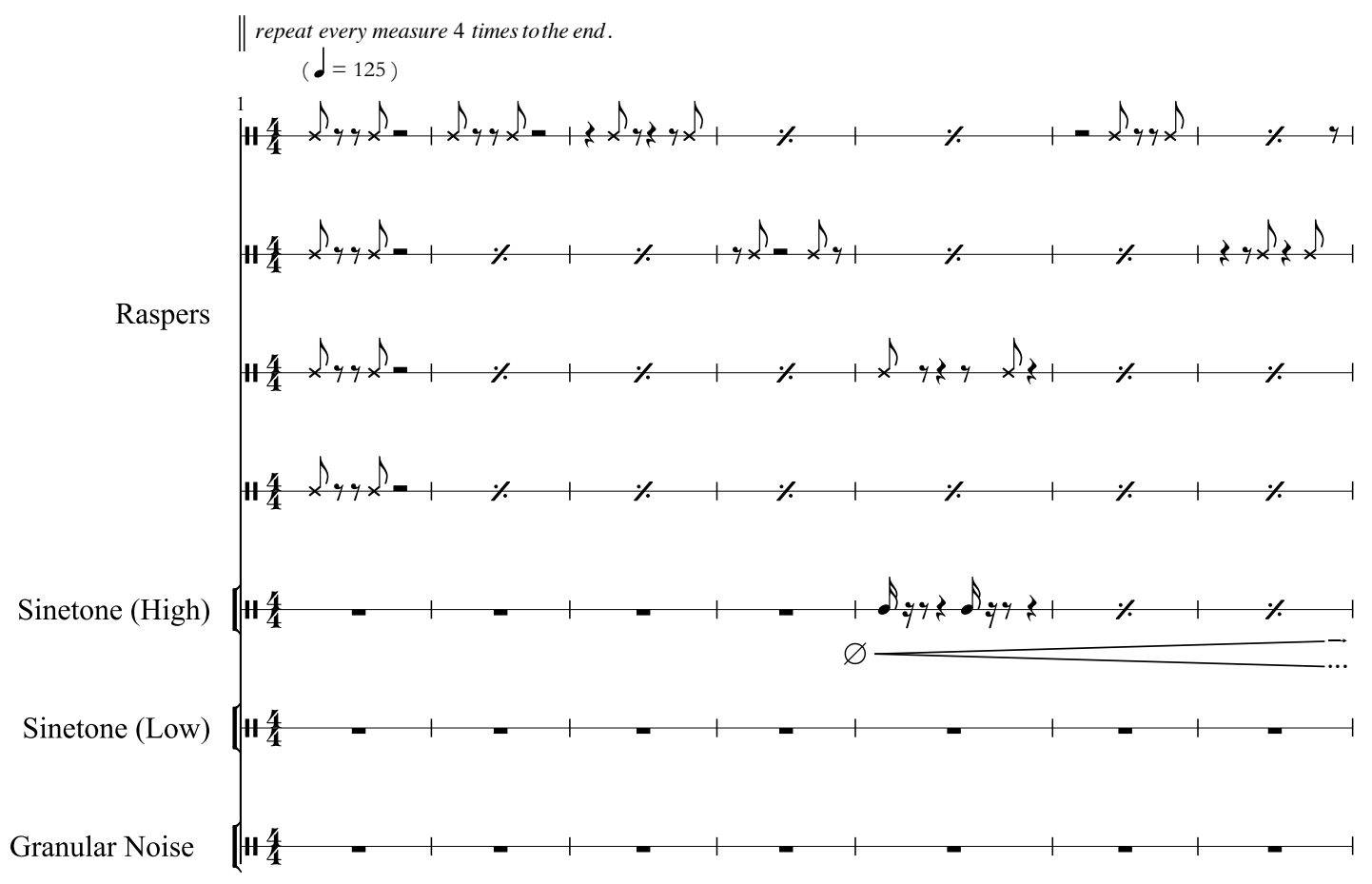



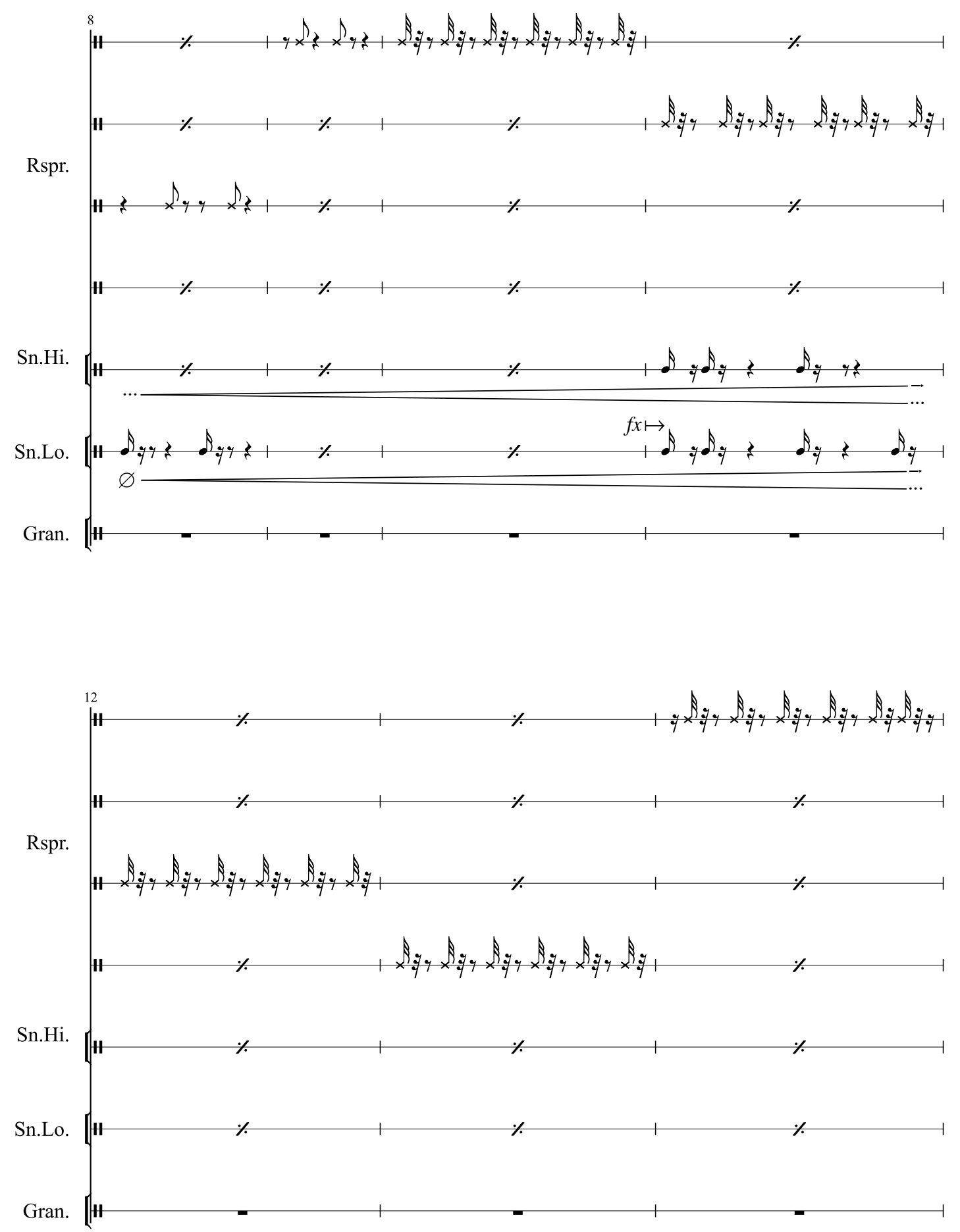

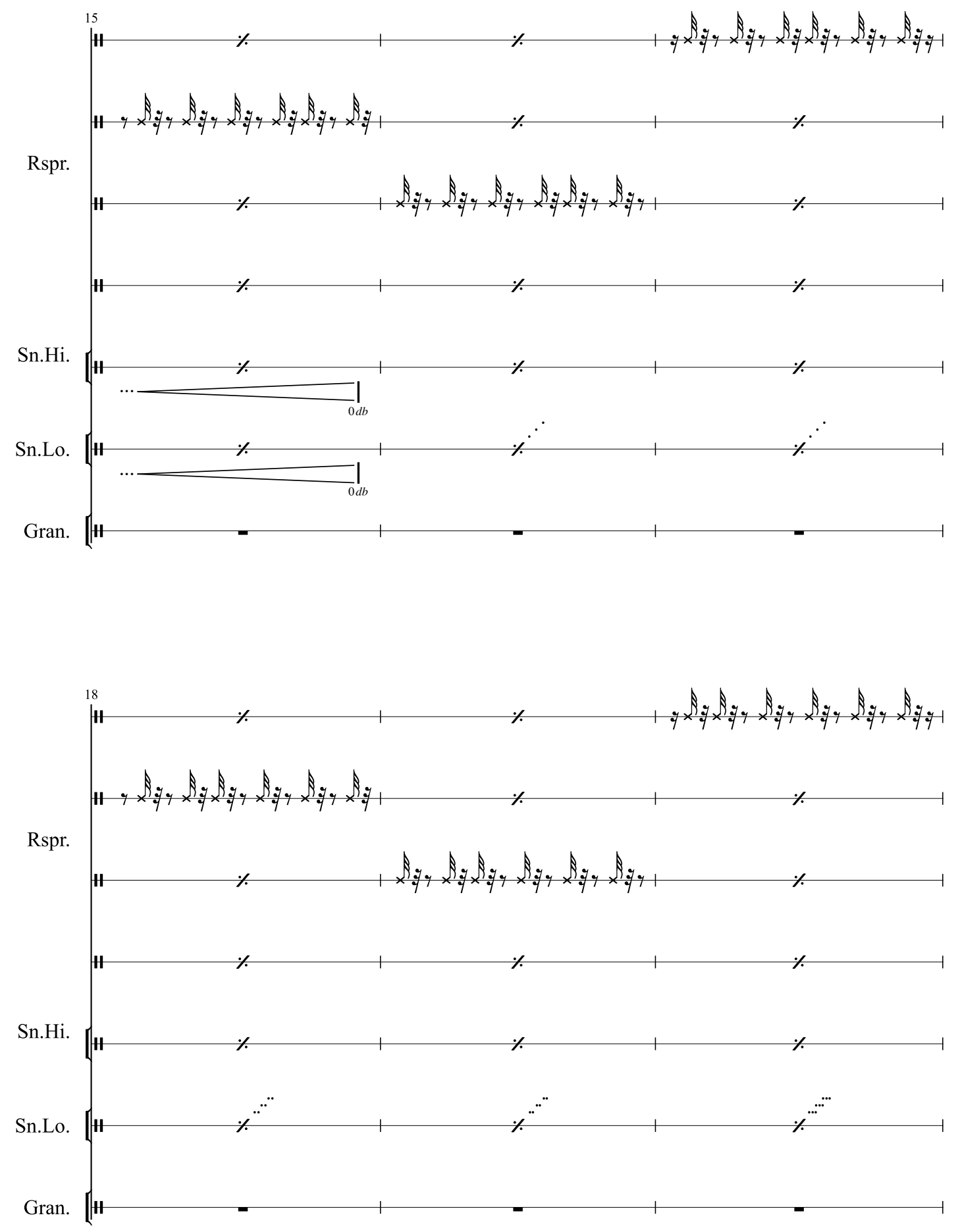

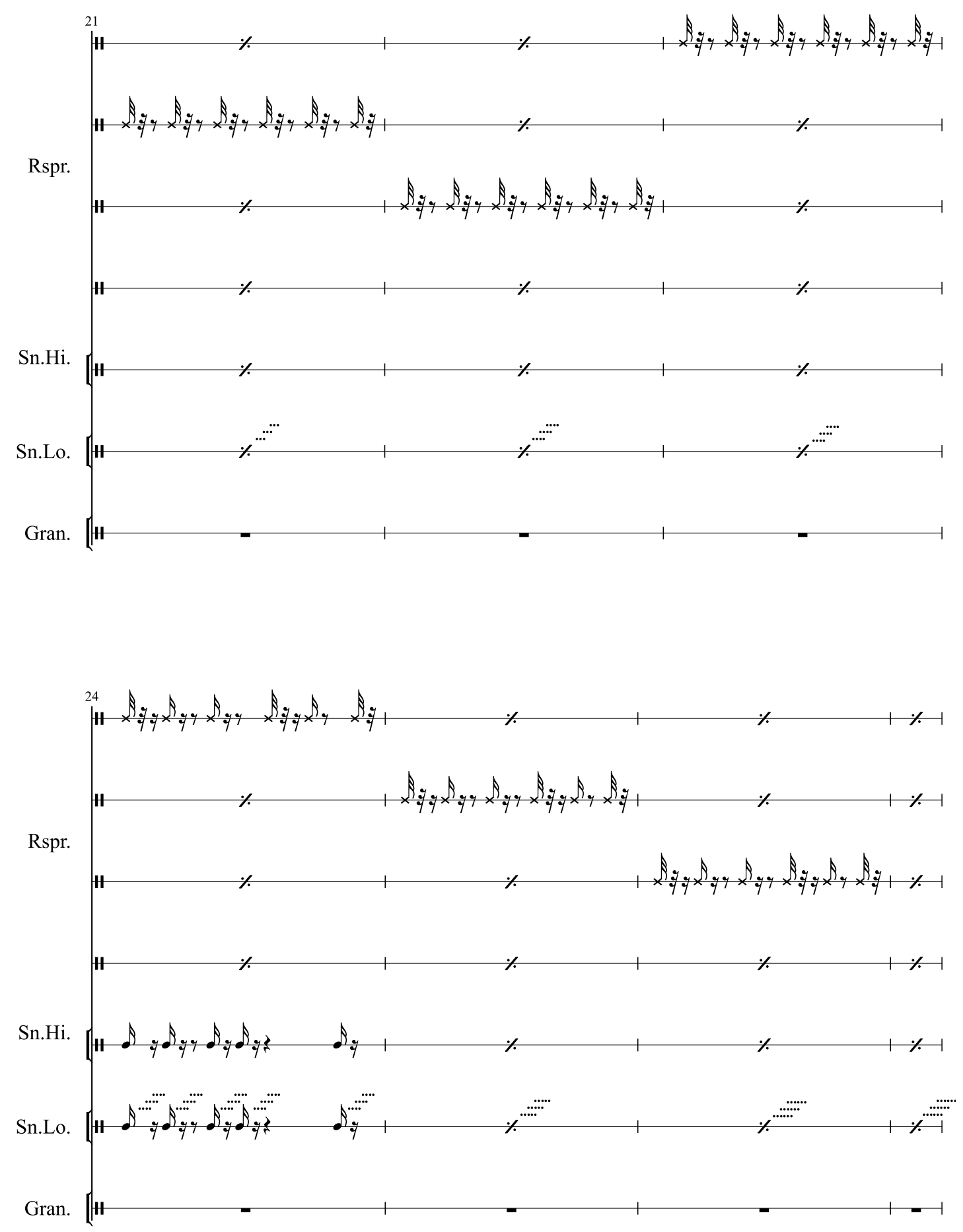

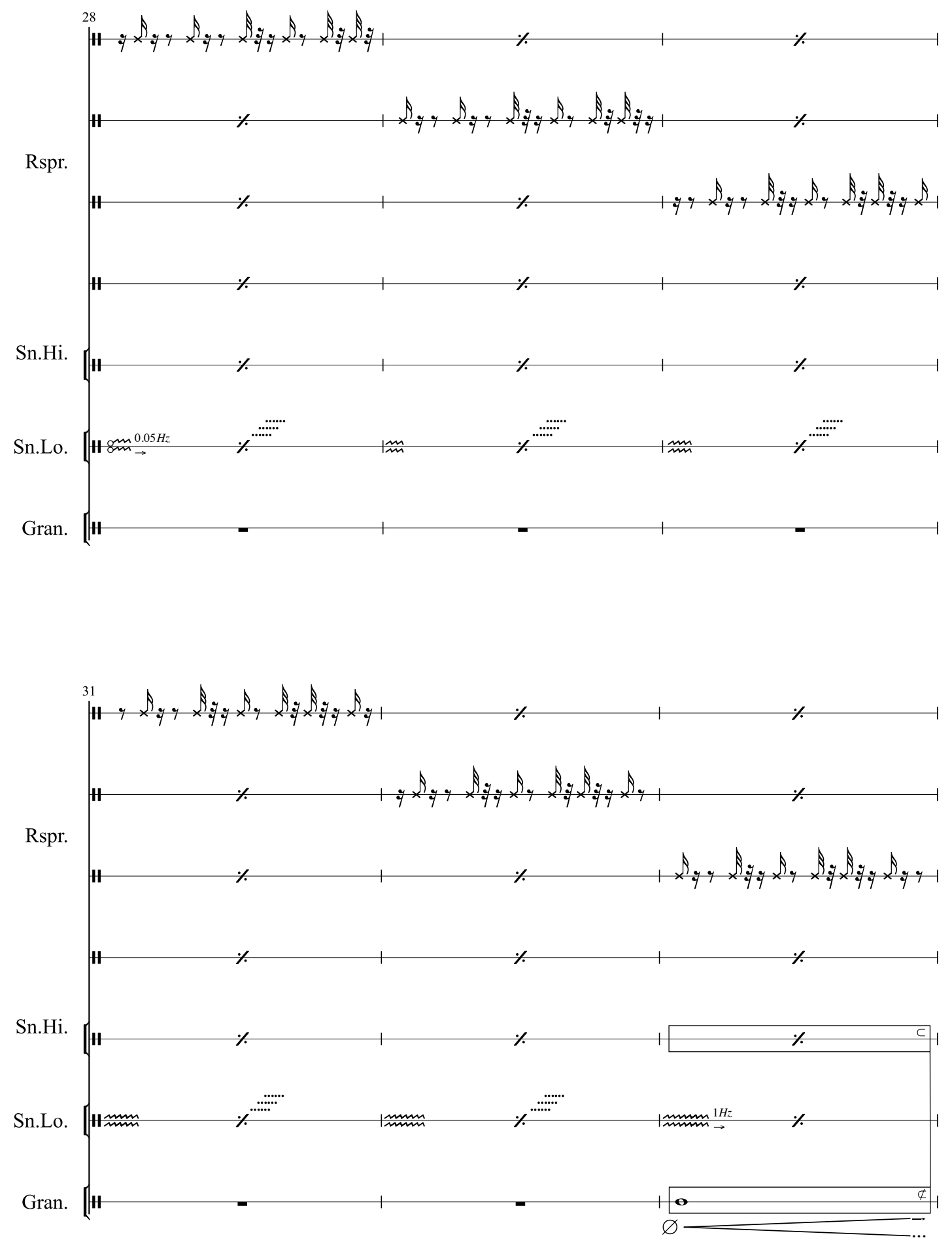

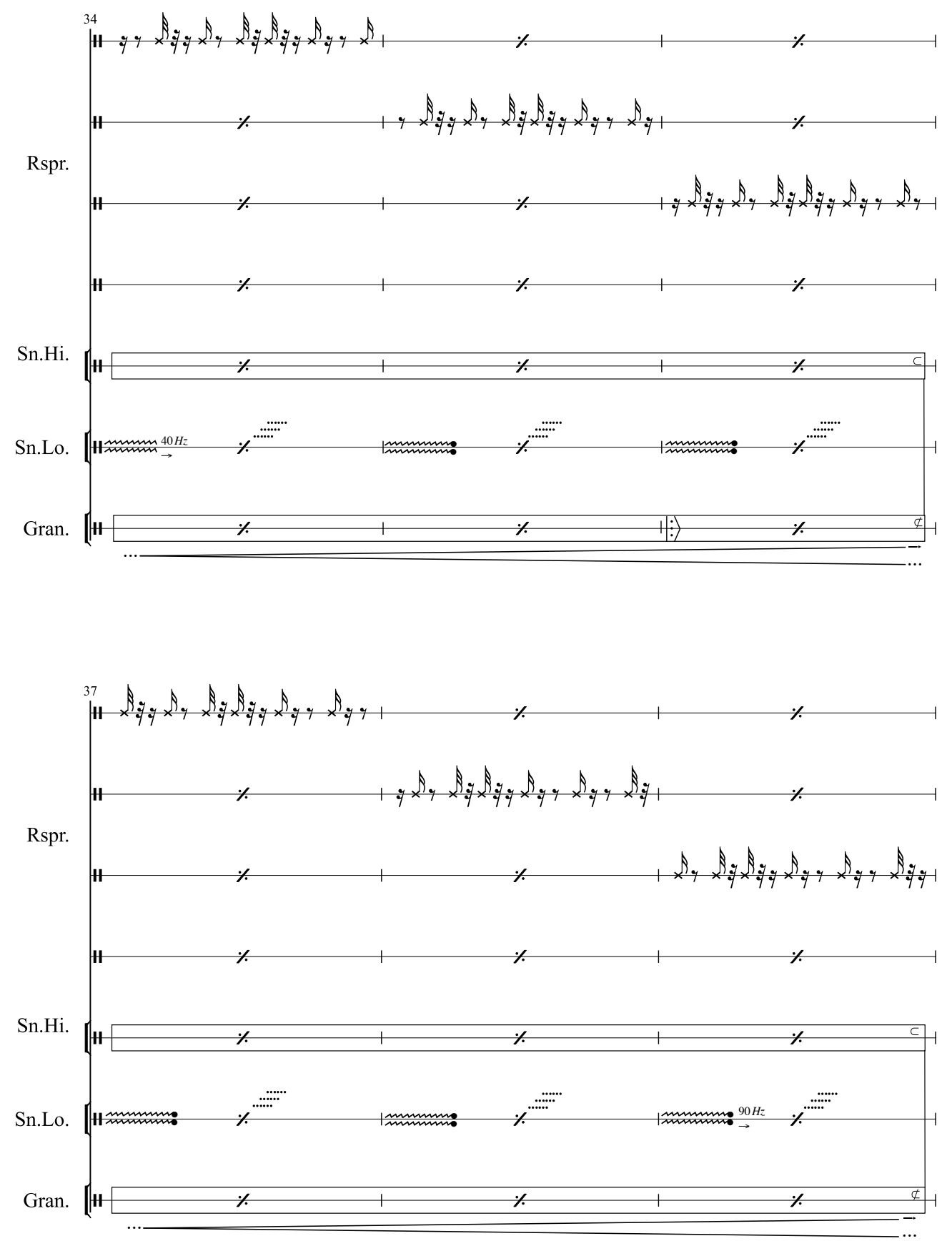

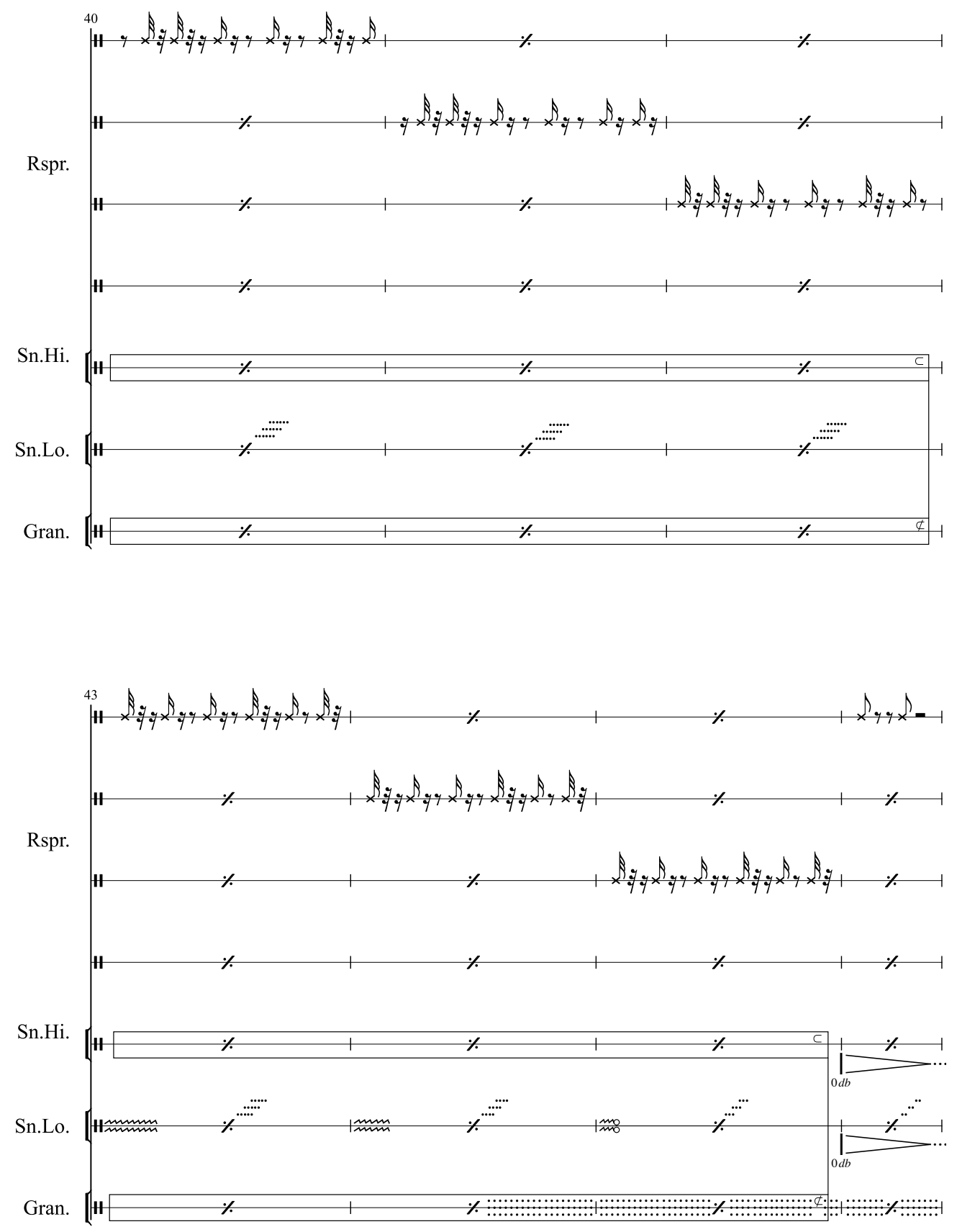


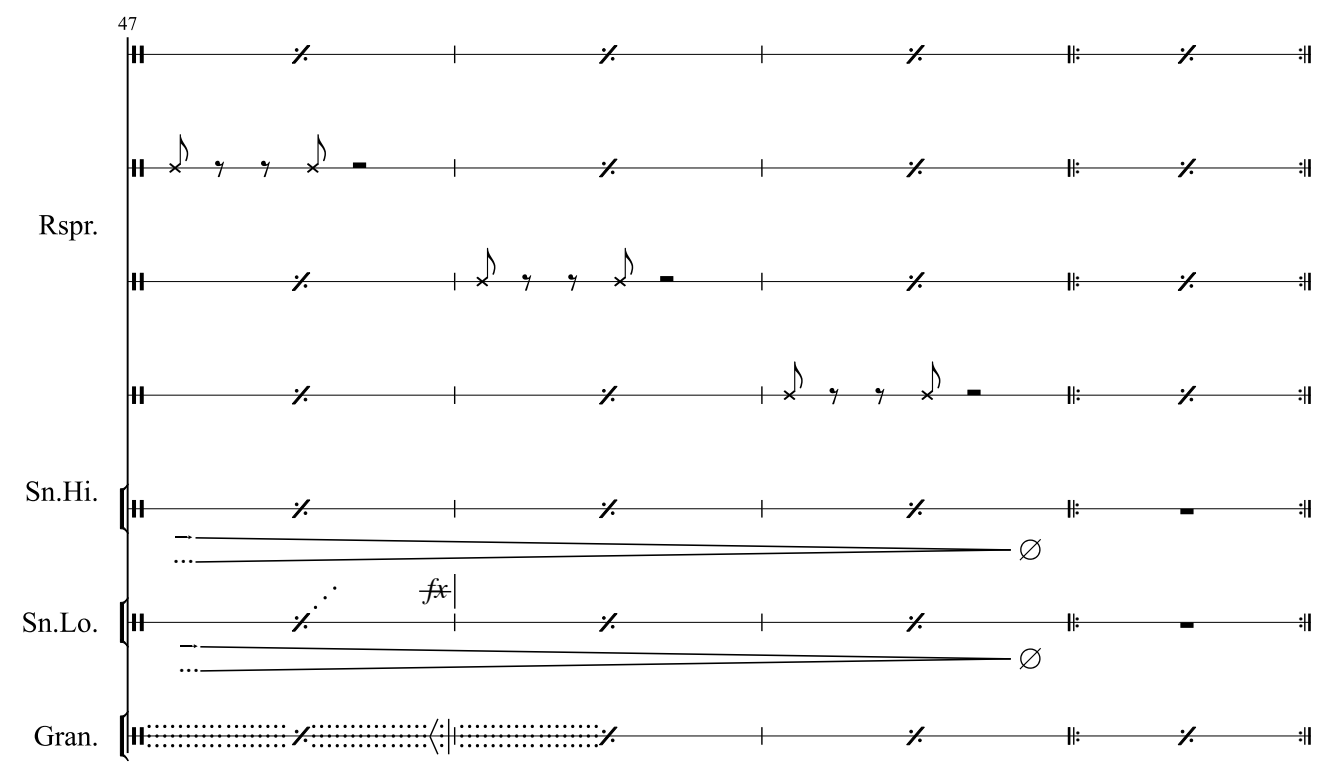




\section{Noise Square (code)}

The following processing code was used in NOISE SQUARE in order to generate the cellular automaton patterns. This code converts the cell-states of the current generation and the rule-set number into MIDI messages and sends them to a designated MIDI channel, which is used by the sound-sculptures. It also creates a visual representation of a series of 16 consecutive generations (that is a grid of 16 by 4) that is sent to Syphon (a plug-in that enables sharing frames between different software), to be fed into the video projector using another software (Resolume Arena 4). This code is a modified version of the code written by Daniel Shiffman in The Nature of Code [125].

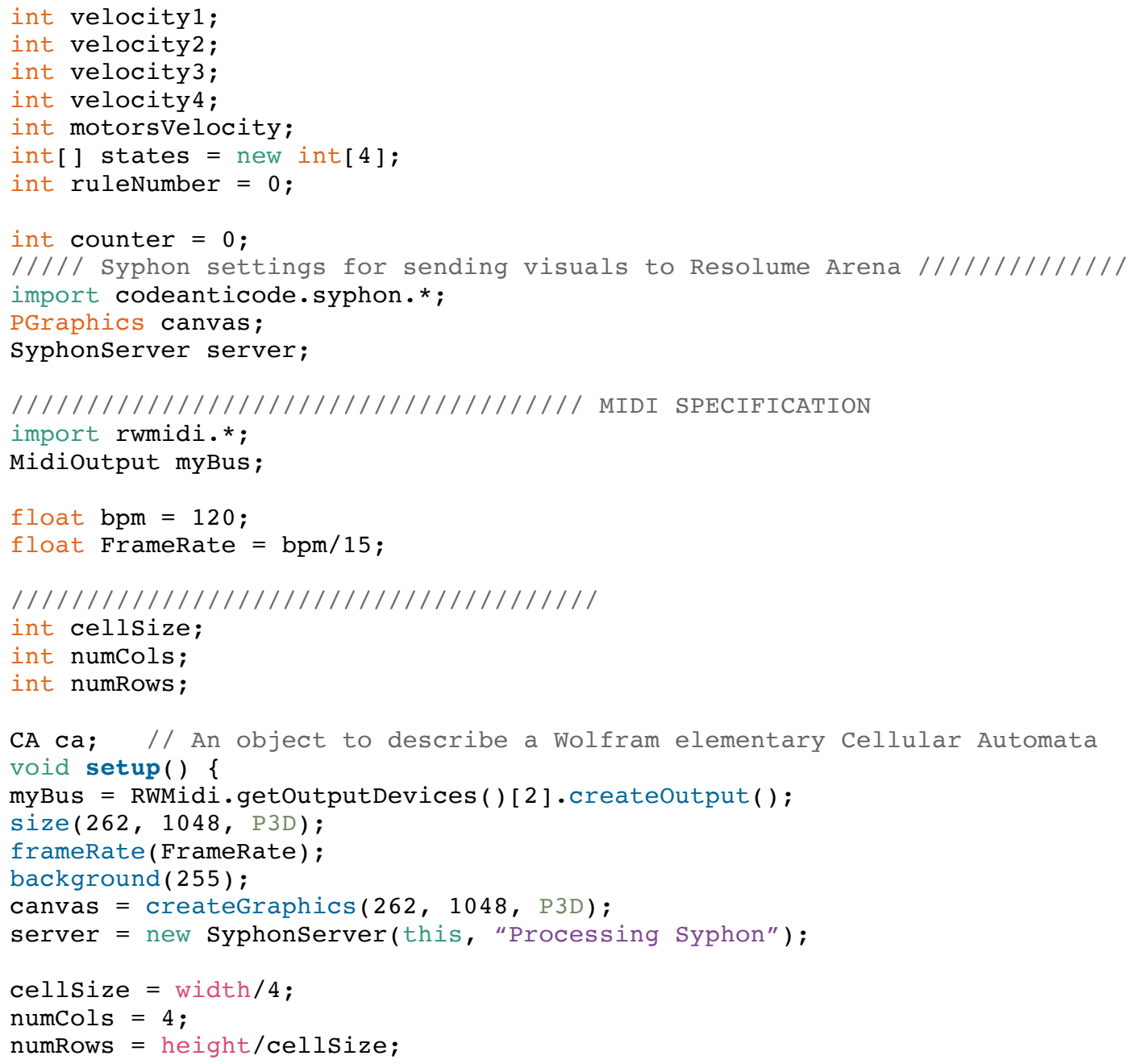




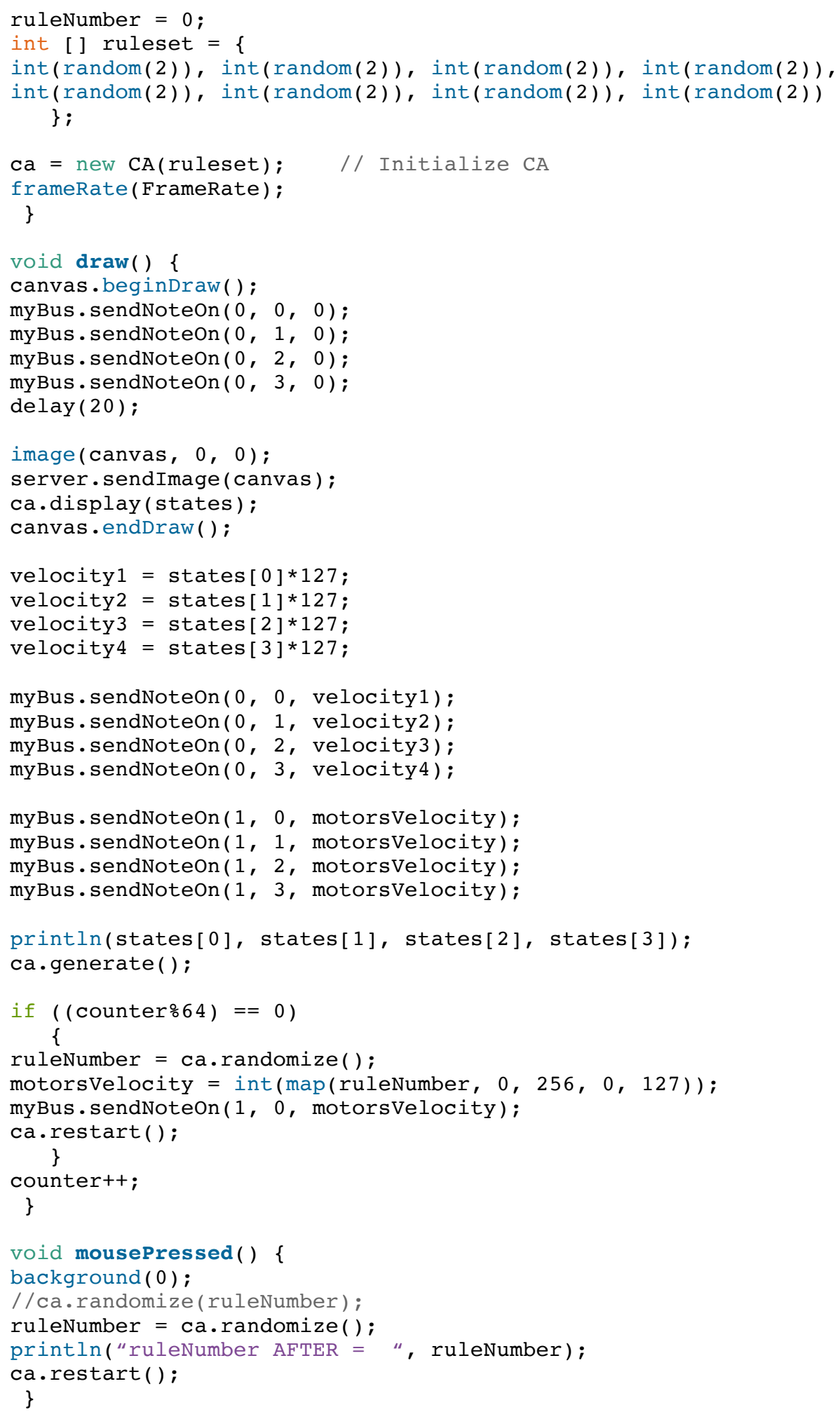




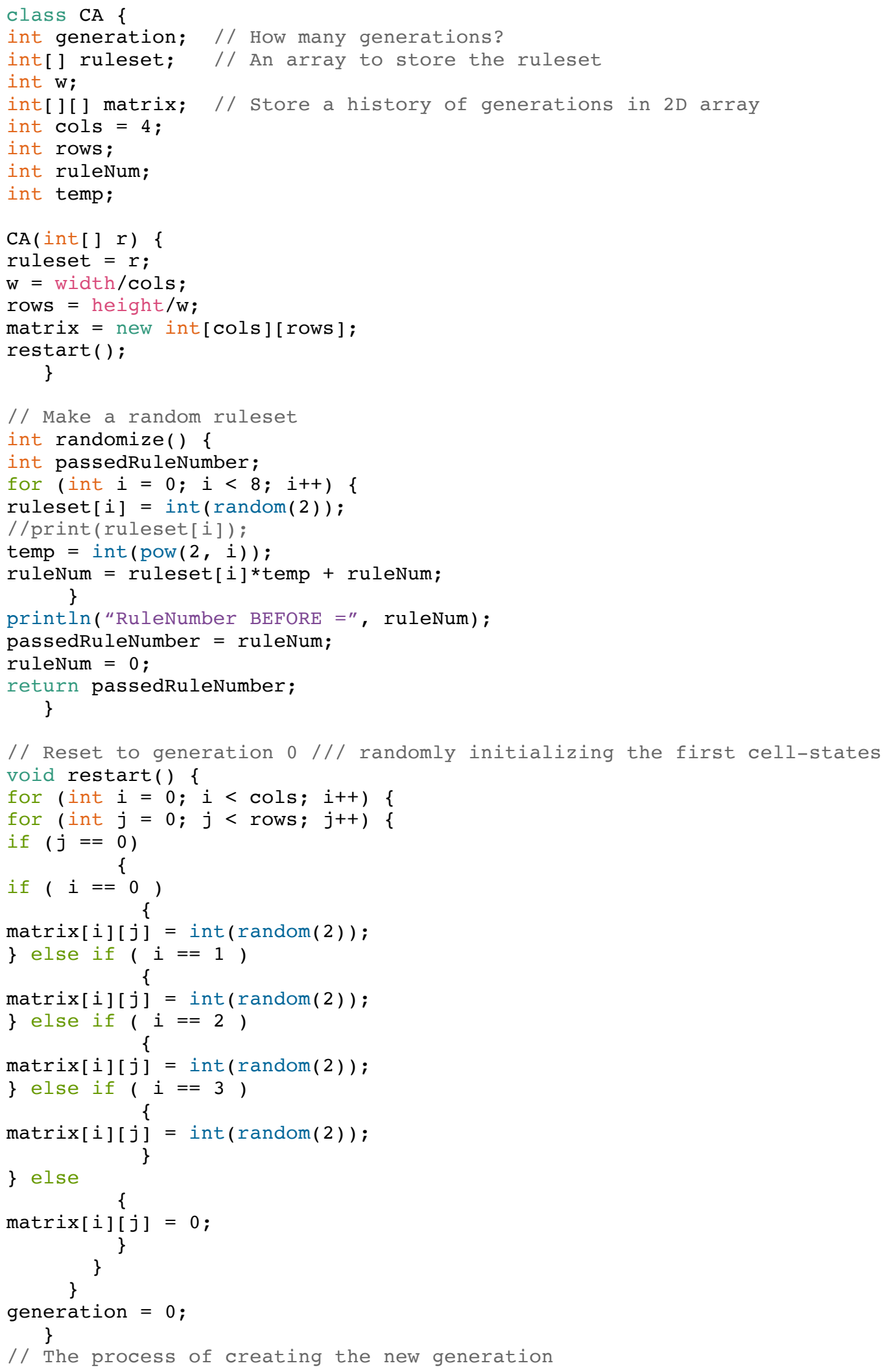




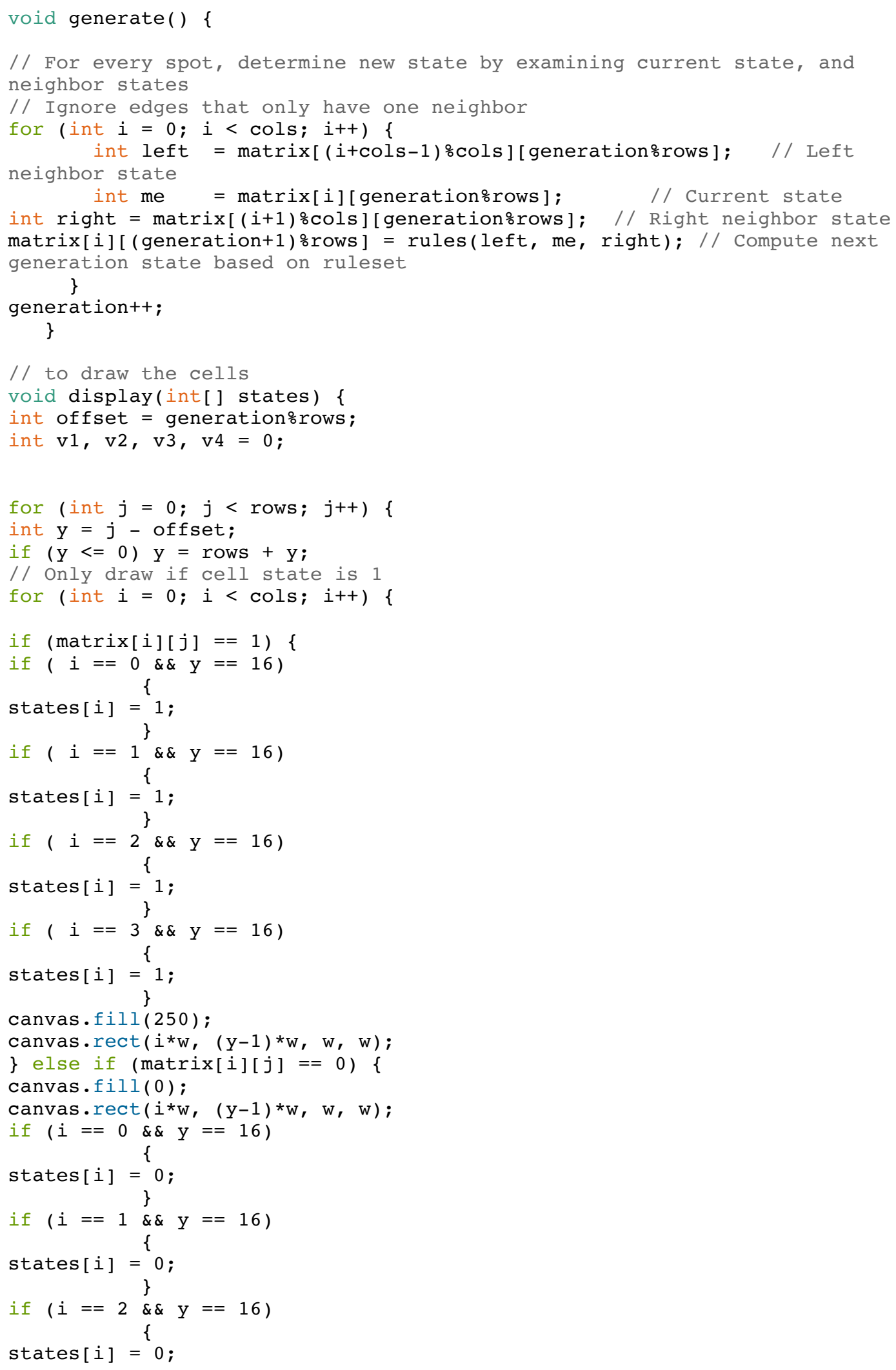




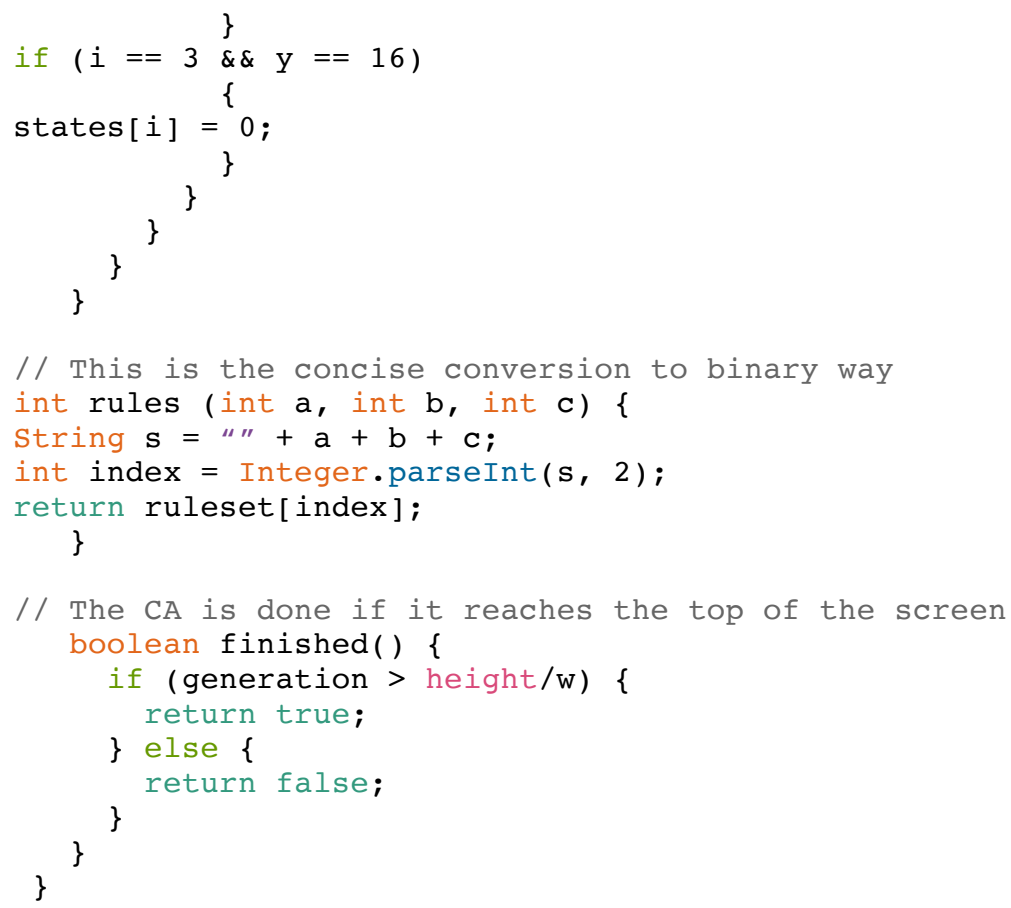




\section{Complex (code)}

The following Processing code was used in complex to map the noise levels of Central Park, Times Square, High Line, and Union Square onto the sound-sculptures. The code sweeps through the RMS data of the designated locations provided by the Citygram dataset, scales them, converts them into MIDI messages, and sends them to the MIDI channel that is used by the sound-sculptures.

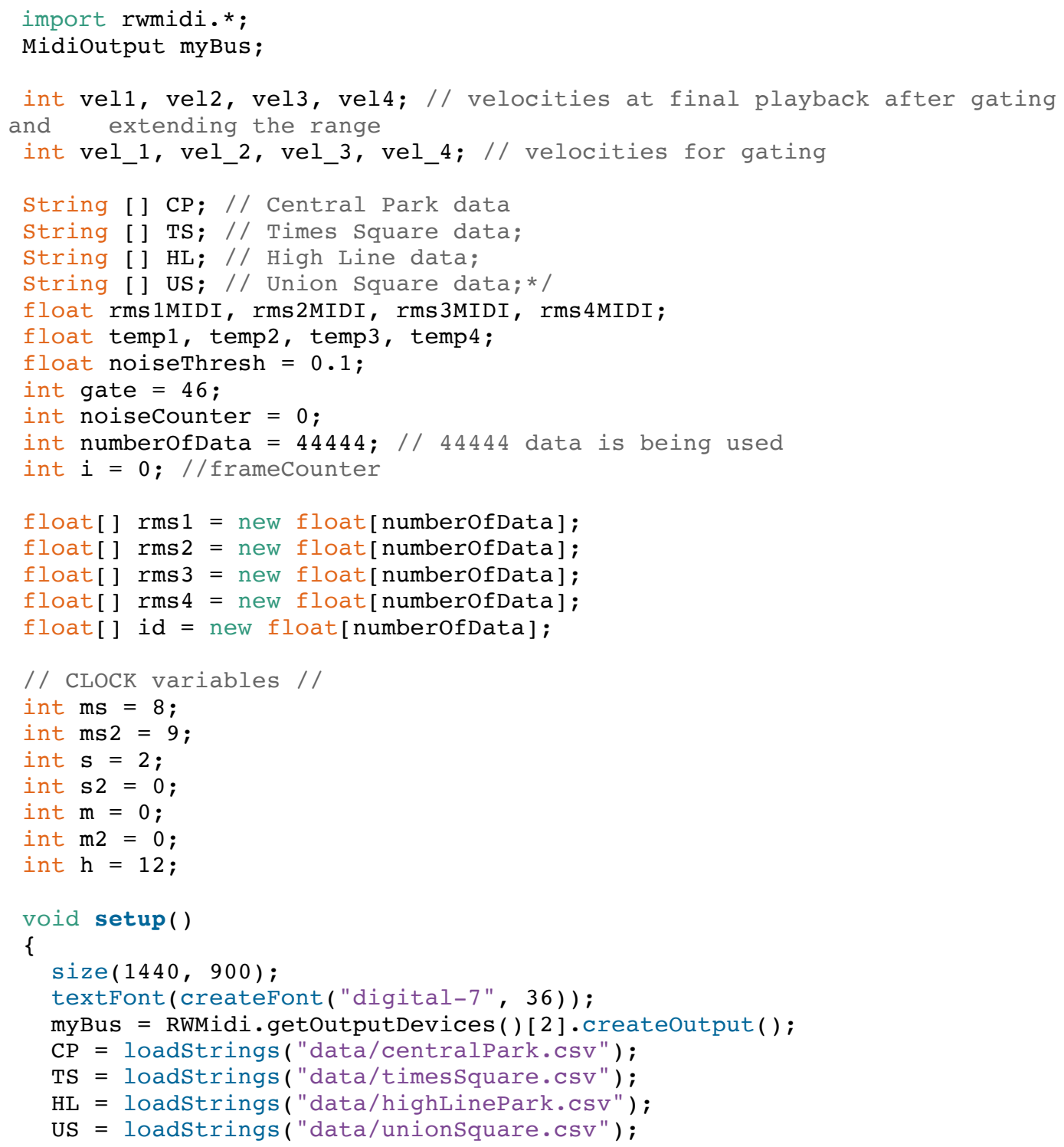




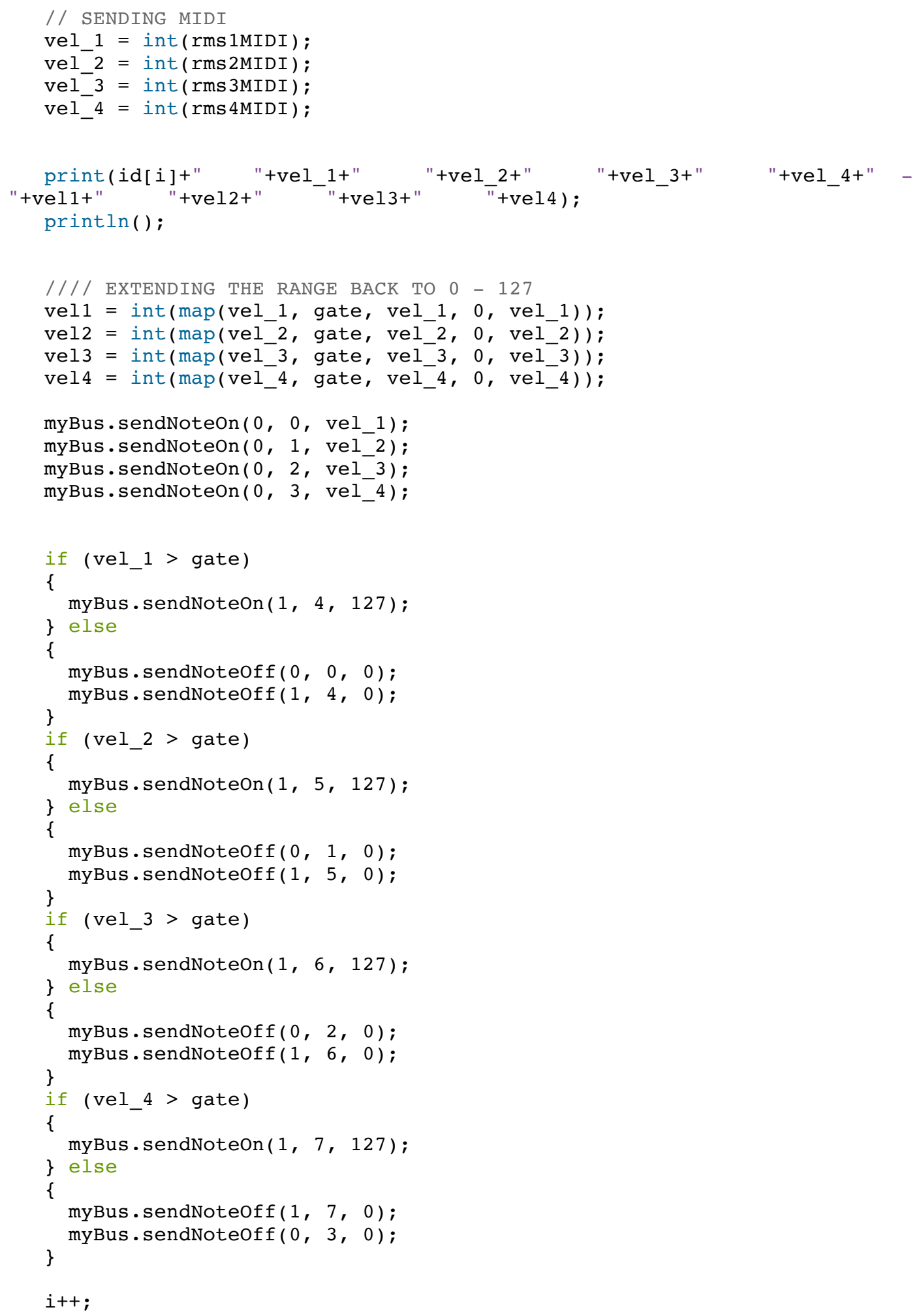




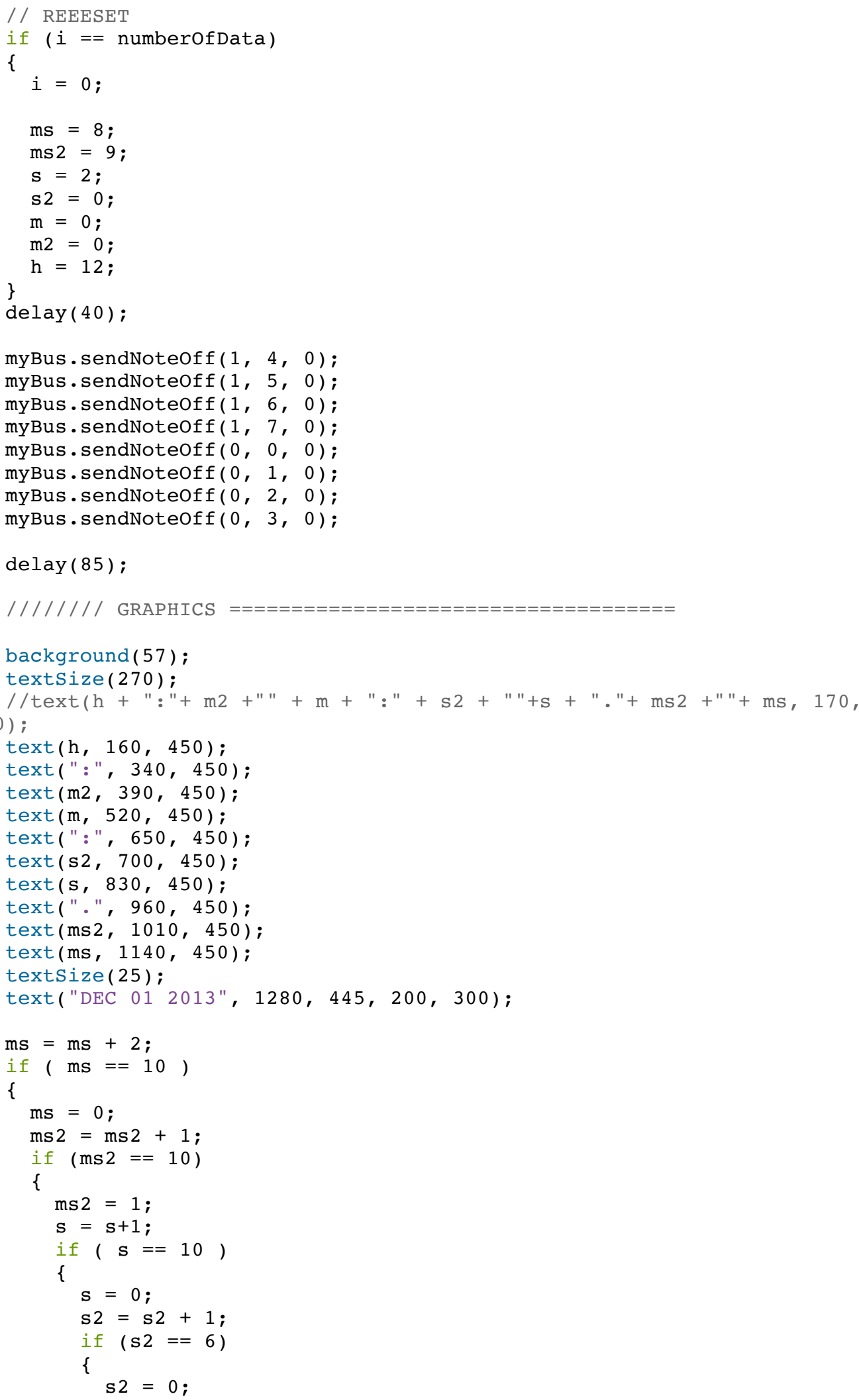




$$
\begin{aligned}
& \mathrm{m}=\mathrm{m}+1 ; \\
& \text { if }(\mathrm{m}==10) \\
& \left\{\begin{array}{l}
\mathrm{m}=0 ; \\
\mathrm{m} 2=\mathrm{m} 2+1 ;
\end{array}\right.
\end{aligned}
$$

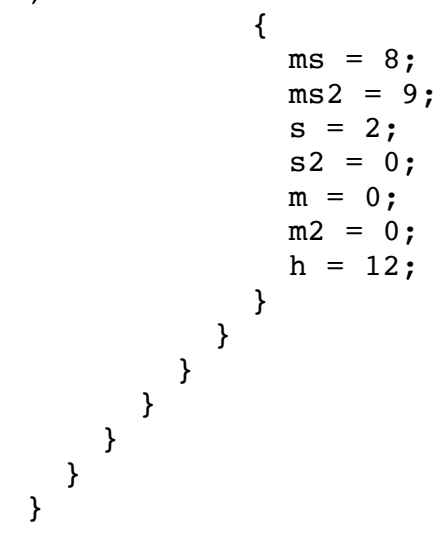




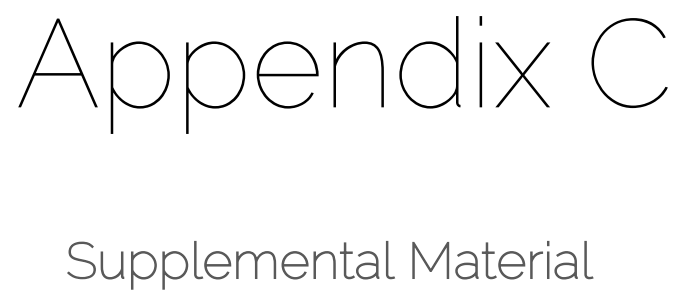

This section provides information on the supplemental material included with this thesis. The supplemental material comprises of eight video files which can be viewed online at www.m-h-z.net/videos, or using the following links:

$\begin{array}{ll}\text { https://youtu.be/ZjzRvJc3Neo } & \text { [video demonstration of Rasper] } \\ \text { https://youtu.be/jxHJLCzwlak } & \text { [video demonstration of Rippler] } \\ \text { https://youtu.be/cSCwSGFjIHE } & \text { [video demonstration of Mutor] } \\ \text { https://youtu.be/U-i0mIlVtCo } & \text { [video documentation of Rasping Music] } \\ \text { https://youtu.be/cMGBwbZxyNA } & \text { [video documentation of NOISE SQUARE] } \\ \text { https://youtu.be/SS6wyMBcfzg } & \text { [video documentation of complex] } \\ \text { https://youtu.be/mNPt8ov1bSA } & \text { [video documentation of Brutalist Noise Ensemble] } \\ \text { https://youtu.be/o2NddNgRRKc } & \text { [video documentation of machine brut(e)] }\end{array}$

